\title{
Proton coupled electron transfer kinetics of redox centers attached to self -assembled monolayers on electrodes
}

Nicholas Madhiri

West Virginia University

Follow this and additional works at: https://researchrepository.wvu.edu/etd

\section{Recommended Citation}

Madhiri, Nicholas, "Proton coupled electron transfer kinetics of redox centers attached to self -assembled monolayers on electrodes" (2006). Graduate Theses, Dissertations, and Problem Reports. 2418.

https://researchrepository.wvu.edu/etd/2418

This Dissertation is protected by copyright and/or related rights. It has been brought to you by the The Research Repository @ WVU with permission from the rights-holder(s). You are free to use this Dissertation in any way that is permitted by the copyright and related rights legislation that applies to your use. For other uses you must obtain permission from the rights-holder(s) directly, unless additional rights are indicated by a Creative Commons license in the record and/ or on the work itself. This Dissertation has been accepted for inclusion in WVU Graduate Theses, Dissertations, and Problem Reports collection by an authorized administrator of The Research Repository @ WVU.

For more information, please contact researchrepository@mail.wvu.edu. 


\title{
Proton Coupled Electron Transfer Kinetics of Redox Centers attached to Self-assembled Monolayers on Electrodes
}

\author{
Nicholas Madhiri \\ Dissertation submitted to the College of Arts and Sciences at West Virginia University \\ in partial fulfillment of the requirements for the degree of \\ Doctor of Philosophy \\ in \\ Chemistry
}

\author{
Harry O. Finklea, PhD., Chair \\ Kenneth C. Showalter, PhD. \\ Fred L. King, PhD. \\ Ronald B. Smart, PhD. \\ John W. Zondlo, PhD. \\ C. Eugene Bennett Department of Chemistry \\ Morgantown, West Virginia \\ 2006
}

\begin{abstract}
Keywords: proton-coupled electron transfer, cyclic voltammetry, self-assembled monolayers, transfer coefficient, reorganization energy, kinetic isotope effect, Marcus DOS theory, concerted mechanism, stepwise model, alkanethiols, heterogenous electron transfer, deuterated buffer.
\end{abstract}

Copyright 2006 Nicholas Madhiri 


\section{ABSTRACT \\ Proton Coupled Electron Transfer Kinetics of Redox Centers attached to Self- assembled Monolayers on Electrodes}

\section{Nicholas Madhiri}

Proton-coupled electron transfer (PCET) reactions play an important role in many biochemical systems and have been focus of great interest recently. These reactions can be represented by a general equation:

$$
\mathrm{Ox}+\mathrm{ne}^{-}+\mathrm{hH}^{+} \leftrightarrow \mathrm{Red}
$$

Traditionally, these reactions have been studied by applying predictions from the stepwise model developed by E. Laviron in the early 1980s. This model is based upon treating the proton transfer step and electron transfer step as discrete steps. Proton transfer is assumed to be at equilibrium under all conditions. However, there is also evidence suggesting a concerted mechanism in which the electron and proton are transferred simultaneously.

In this study, the predictions of the two models are tested by tethering an osmium complex, $\mathrm{Os}^{\mathrm{II}}(\mathrm{bpy})_{2}(\mathrm{py})\left(\mathrm{OH}_{2}\right)$, (bpy = bipyridine and py = 4-Aminomethylpyridine) to an electrode using self assembled monolayers. Data analysis is carried out using Cyclic Voltammetry. Results obtained show that the osmium system follows the thermodynamic model closely. However, kinetically, the system deviates substantially from predictions of the stepwise model. The standard rate constant and the transfer coefficient are weakly dependent on $\mathrm{pH}$. Tafel plots are asymmetrical at all $\mathrm{pHs}$. The transfer coefficient at zero overpotential is consistently less than the 0.5 value expected for simple electron transfer. Comparison of results from this study to earlier work by Haddox reveals that the standard rate constant decreases by a factor of 10 when the diluent chain length is increased by four methylene groups. The stepwise model cannot explain these observations.

The concerted mechanism is tested by measuring kinetic parameters in deuterated electrolytes, to investigate the kinetic isotope effect. The weak, but noticeable dependence of the standard rate constant on $\mathrm{pH}$ is interpreted in terms of a concerted mechanism, with short proton tunneling distance. The reorganization energy of the Os ${ }^{\mathrm{II}}$ species is higher than that of the Os ${ }^{\mathrm{III}}$ form. This is contrary to expectations. Based on electrostatic arguments, the opposite would be expected since the higher oxidation state has higher bond vibration frequency. Another striking observation is the sudden break in the plot of standard rate constant vs pD. A third striking observation is the inverse correlation of the standard rate constant with the reorganization energy of Os ${ }^{\mathrm{II}}$. An attempt to explain the results using the concerted model was limited by the absence of a fully developed theoretical model. 


\section{ACKNOWLEDGEMENTS}

This work would not be possible without the excellent intellectual and professional mentorship I received from my advisor Dr Harry O. Finklea. I would like to thank him for his unwavering support throughout the course of this study. He did not only allow me to independently conduct my research, but kept me focused in the right direction to achieve the research goals. I learnt so much from him both from an academic standpoint as well as from the way he conducts himself professionally. His professional conduct and hard work will go a long way in my life. I feel quite privileged and honored to be among the students who have had a chance to tap from his reservoir of knowledge and wisdom. I would also like to thank Dr Kenneth Showalter, Dr Fred King, Dr John Zondlo and Dr Ronald Smart for their willingness to serve in my committee and for their constructive comments and advice. Special thanks also go to Dr Reuben Simoyi for introducing me to WVU and for giving me a chance to pursue a professional career. My appreciation for everything he has done in my life. God bless his generous heart.

Much appreciation goes to my wife and close ally, Embedzayi, for being there for me when no one else could. Thank you so much for having confidence and faith in my abilities. Thank you dear for being a continuous source of energy and for all the emotional support. To my son Ryan, I know I cannot make up for lost time during these busy moments, but I promise to be there for you son. Thank you for your patience. To my parents and my in-laws, thank you all for your support throughout this long journey. Life is a journey that requires the company of the right people, many thanks to friends who were a constant source of fun and encouragement. Of special mention is Dr Tsanangurayi Tongesayi and Innocent Pumure. Thank you guys for your moral support. Lastly, and most importantly, my faith in God kept me going. I was able to face challenges head-on

knowing that "I can do all things through Christ who strengthens me"(Phillipians 4:13). Praise be to GOD, the most High, forever. 


\section{TABLE OF CONTENTS}

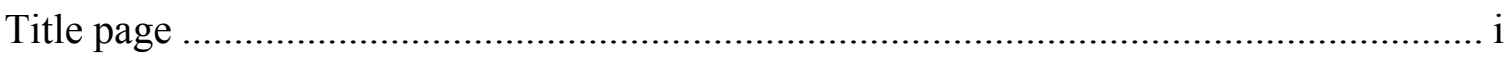

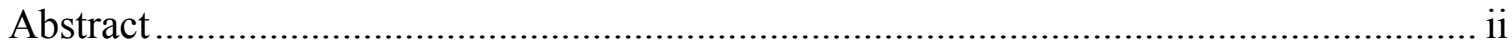

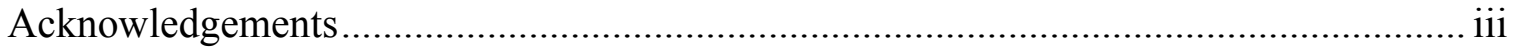

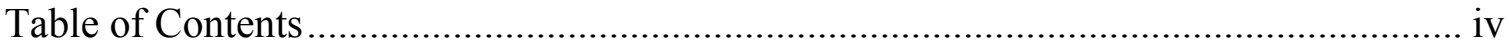

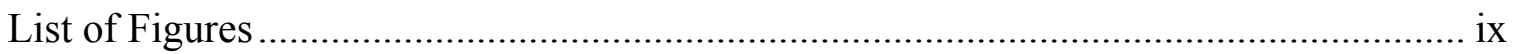

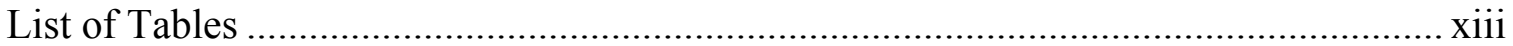

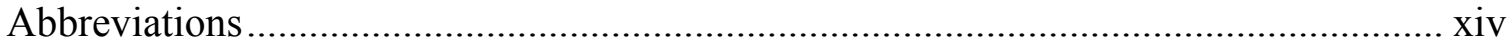

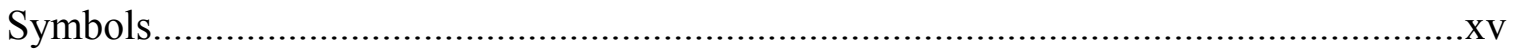

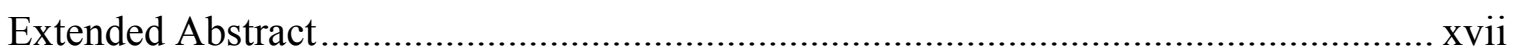

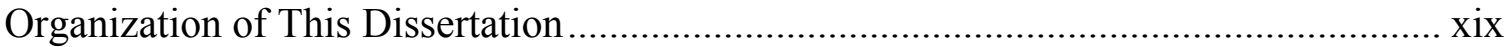


Chapter 1: Introduction to Simple Electron Transfer Theory .....................................1

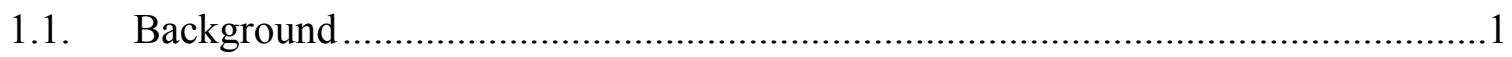

1.2. Limitations of the Butler-Volmer Theory …….....................................................12

1.3. Physical meaning of the Standard Rate Constant .................................................12

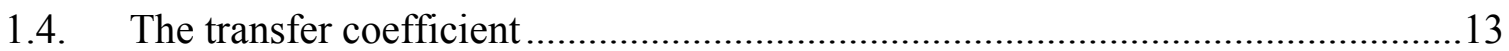

1.5. Marcus Density of States Model: A closer look …………....................................

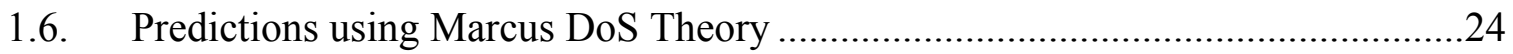

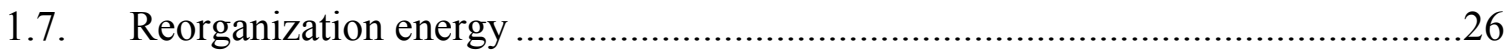

1.8. Data interpretation \& treatment using Marcus model when the reorganization

energies for the reduced and oxidized species are differet

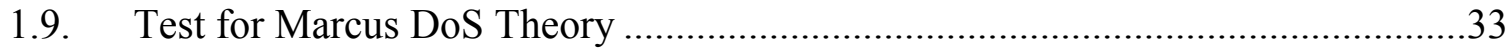

Chapter 2: Introduction to Self-Assembled Monolayers............................................36

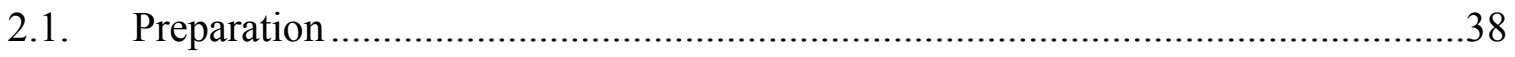

2.2. Electron transfer on SAM modified electrodes .................................................41

2.3. Review of electron transfer kinetic measurements using mixed SAMs ................45 


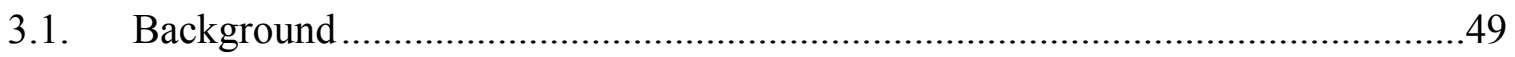

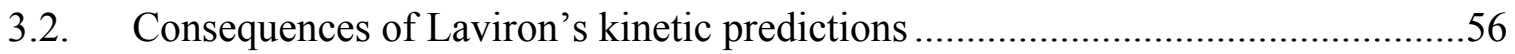

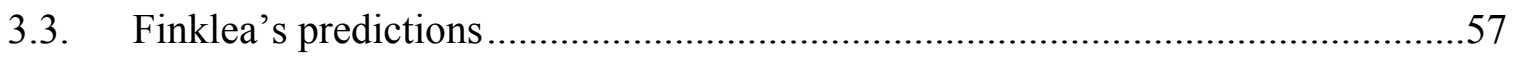

Chapter 4: The Osmium System .....................................................................661

4.1. Synthesis of polypyridyl osmium complexes ............................................61

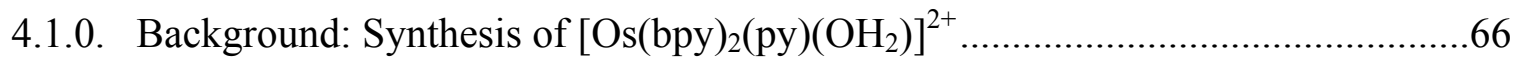

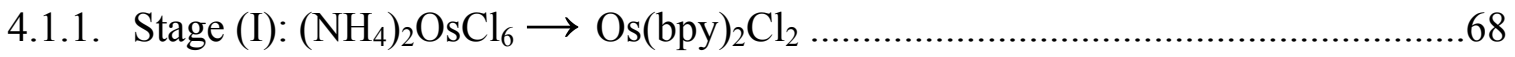

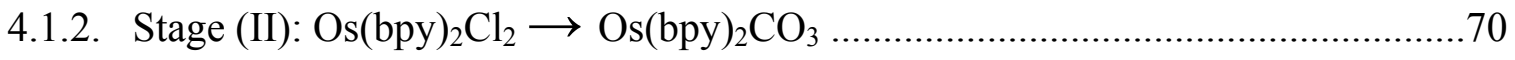

4.1.3. Stage (III): Os(bpy $)_{2} \mathrm{CO}_{3} \rightarrow\left[\mathrm{Os}(\mathrm{bpy})_{2}\left(\mathrm{OH}_{2}\right)_{2}\right]^{2+} \rightarrow\left[\mathrm{Os}(\mathrm{bpy})_{2}(\mathrm{py})\left(\mathrm{OH}_{2}\right)\right]^{2+} \ldots . . .76$

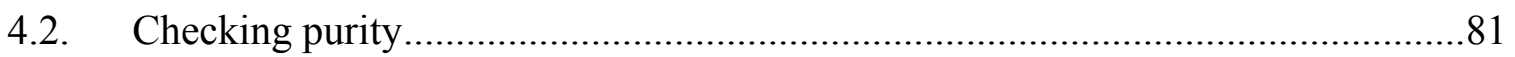




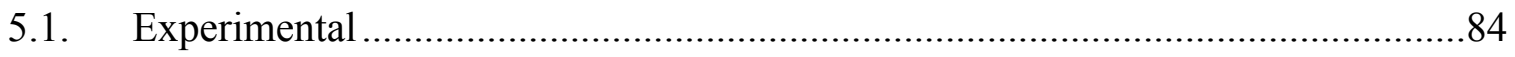

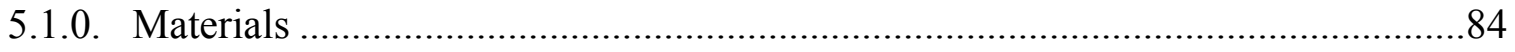

5.1.1. Preparation of self-assembled monolayers ................................................ 84

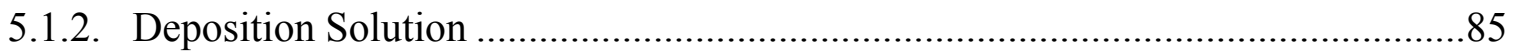

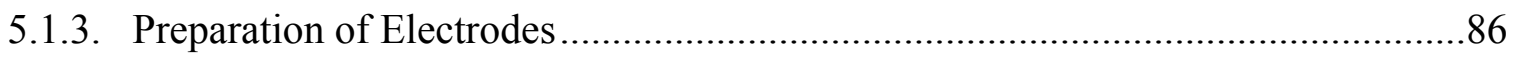

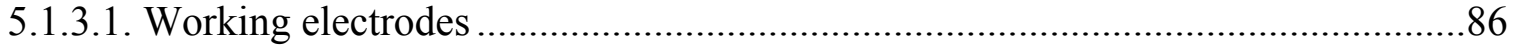

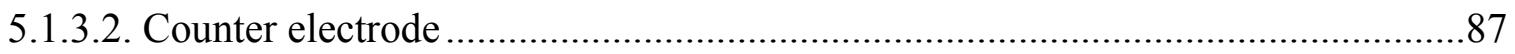

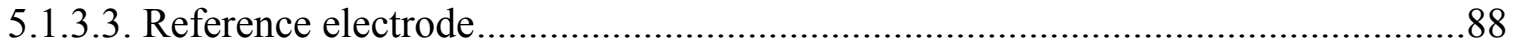

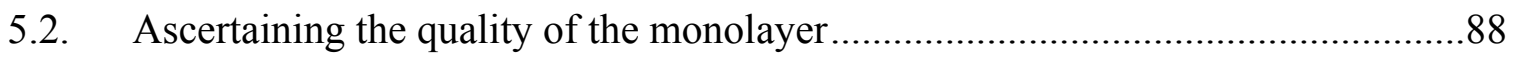

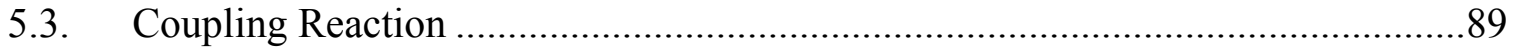

5.4. Thermodynamic information of Redox molecules attached to SAMs.................92

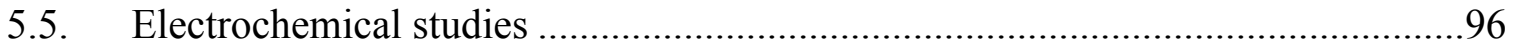

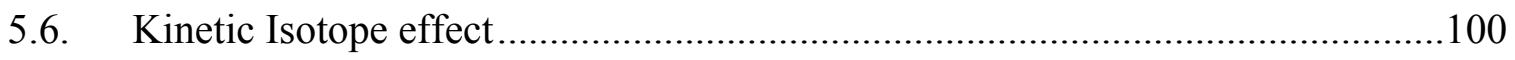

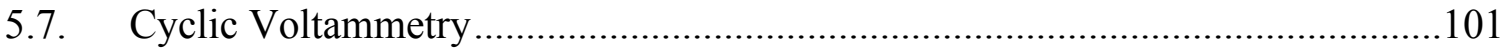

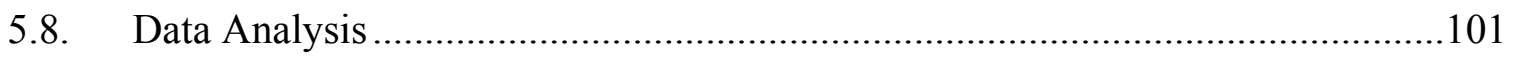

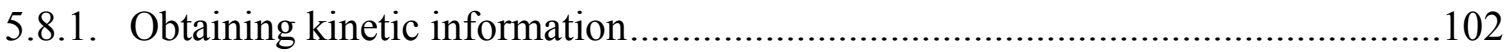




\section{Chapter 6: Proton Coupled Electron Transfer: Kinetic Studies of Polypyridyl}

Osmium

6.1. Results 106

6.1.1. Transfer coefficient vs $\mathrm{pH}$

6.1.2. Standard rate constant vs chain length 128

6.1.3. Slope vs $\mathrm{pH}$

6.1.4. Reorganization energy vs $\mathrm{pH}$

Chapter 7: Discussion. .136

7.1. Proposed mechanism 136

7.2 Conclusion 140

7.3 Future Work 142

References: 


\section{List of Figures}

Figure 1.1 Changes in potential energy during a redox reaction at an electrode .........6

Figure 1.2 Effects of potential on the energy barrier for a simple redox process .........7

Figure 1.3 An expanded view of the intersection region shown by the dotted box in

Figure 1.2 ................................................................................ 9

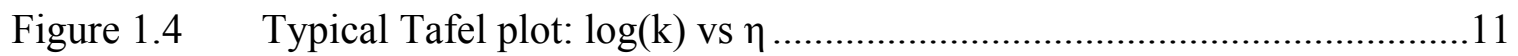

Figure 1.5 Potential dependence of transfer coefficient as predicted by the parabolic

free energy curves with equal curvature

Figure 1.6 Density of states diagram showing the Gaussian distribution of redox

species $(\mathrm{Ox})$ and the Fermi distribution function

Figure 1.7 Dependence of cathodic rate constant on overpotential

Figure 1.8 Theoretical Tafel plots at various reorganization energies

Figure 1.9 Generic variation of free energy with reaction coordinate for a simple

electron transfer half reaction .28

Figure 1.10 Tafel Plot for different reorganization energies

Figure 1.11 $\alpha$ vs $\eta$ for a case of different reorganization energies 
Figure 2.1 Electroactive vs Blocking SAMs

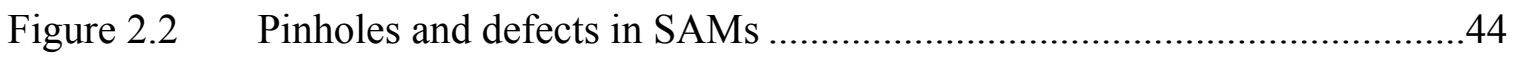

Figure $3.1 \quad 1 \mathrm{e}, 1 \mathrm{H}$ square scheme I for PCET reactions .............................................52

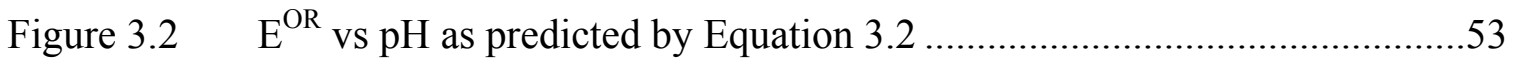

Figure 3.3 Theoretical predictions using Laviron and Finklea models; variation of

standard rate constant with $\mathrm{pH}$

Figure 3.4 Potential dependence of transfer coefficient, effect of reorganization

energy.......

Figure 3.5 Theoretical predictions using Finklea model; variation of the transfer

coefficient with $\mathrm{pH}$

Figure 4.1 Pourbaix diagram for $\mathrm{Os}^{(\mathrm{V} / \mathrm{IV})}$ and $\mathrm{Os}{ }^{(\mathrm{IV} / \mathrm{III})}$ redox couples of

$\left[\mathrm{Os}(\text { tpy })(\mathrm{bpy})\left(\mathrm{OH}_{2}\right)\right]^{2+}$

Figure 4.2 Structure of $\left[\mathrm{Os}(\mathrm{bpy})_{2}(\mathrm{py})\left(\mathrm{OH}_{2}\right)\right]^{2+}$

Figure 4.3 Scheme of general synthetic strategy for $\left[\mathrm{Os}(\mathrm{bpy})_{2}(\mathrm{py})\left(\mathrm{OH}_{2}\right)\right]^{2+}$ .67

Figure 4.4 UV spectrum of $\mathrm{Os}(\mathrm{bpy})_{2} \mathrm{Cl}_{2}$ in $\mathrm{CH}_{3} \mathrm{CN}$ .69 
Figure 4.5 Cyclic Voltammogram of $\mathrm{Os}(\mathrm{bpy})_{2} \mathrm{Cl}_{2}$ in tetramethylammonium

tetrafluoroborate/ $\mathrm{CH}_{3} \mathrm{CN}$ on a bare electrode

Figure 4.6 Cyclic Voltammogram of $\left[\mathrm{Os}(\mathrm{bpy})_{2}\left(\mathrm{OH}_{2}\right)_{2}\right]^{2+}$ from Os(bpy $)_{2} \mathrm{CO}_{3}$

Figure 4.7 Cyclic Voltammogram of cis- $\left[\mathrm{Os}(\mathrm{bpy})_{2}\left(\mathrm{OH}_{2}\right)_{2}\right]^{2+}$ from $\mathrm{Os}(\mathrm{bpy})_{2} \mathrm{CO}_{3} \ldots . . . .73$

Figure 4.8 UV spectrum of $\mathrm{Os}(\mathrm{bpy})_{2} \mathrm{CO}_{3}$ in $\mathrm{CH}_{3} \mathrm{CN}$ .75

Figure 4.9 UV spectrum of $\left[\mathrm{Os}(\mathrm{bpy})_{2}(\mathrm{py})\left(\mathrm{OH}_{2}\right)\right]^{2+}$ .79

Figure 4.10 CVs for $\left[\mathrm{Os}(\mathrm{bpy})_{2}(\mathrm{py})\left(\mathrm{OH}_{2}\right)\right]^{2+}$ showing both $\mathrm{Os}{ }^{\mathrm{III}} / \mathrm{Os}^{\mathrm{II}}$ wave and $\mathrm{Os}^{\mathrm{IV}} / \mathrm{Os}^{\mathrm{III}}$ .80

Figure 4.11 Variation of formal potential with $\mathrm{pH}$ for $\left[\mathrm{Os}(\mathrm{bpy})_{2}(\mathrm{py})\left(\mathrm{OH}_{2}\right)\right]^{2+}$ in $0.5 \mathrm{M}$

$\mathrm{K}_{2} \mathrm{SO}_{4} / 0.1 \mathrm{M}$ Britton-Robinson buffer on a bare electrode

Figure 5.1 Reversible CV showing faradaic and non-faradaic current .93

Figure 5.2 Generalized diagram of experimental set up .97

Figure 6.1 Typical CV for $\left[\mathrm{Os}(\mathrm{bpy})_{2}(\mathrm{py})\left(\mathrm{OH}_{2}\right)\right]^{2+}$ attached to a (90/10)

$\mathrm{HS}\left(\mathrm{CH}_{2}\right)_{15} \mathrm{COOH} / \mathrm{HS}\left(\mathrm{CH}_{2}\right)_{15} \mathrm{CH}_{2} \mathrm{OH}$ on an electrode 107

Figure 6.2 Variation of formal potential $\mathrm{vs} \mathrm{Ag} / \mathrm{AgCl}$ with $\mathrm{pH}$ and $\mathrm{pD}$. 108

Figure 6.3 Overlay $\mathrm{CV}$ plots at selected $\mathrm{pHs}$ $112-14$ 
Figure 6.4 Variation of peak potential with scan rate at selected $\mathrm{pHs}$

Figure 6.5 Typical Tafel plots at pH 6.0 for $\left[\mathrm{Os}(\mathrm{bpy})_{2}(\mathrm{py})\left(\mathrm{OH}_{2}\right)\right]^{2+}$

Figure 6.6 Potential dependence of the transfer coefficient at $\mathrm{pH} 6.0$ 120

Figure 6.7 Comparison between experimental data and theoretical predictions of the stepwise model

Figure 6.8 Kinetic Isotope effect: $\log \left(\mathrm{k}_{\mathrm{s}}\right)$ vs $\mathrm{pH}$ in $\mathrm{H}_{2} \mathrm{O}$ and $\mathrm{D}_{2} \mathrm{O}$ 124

Figure 6.9 Transfer coefficient at zero overpotential vs $\mathrm{pH}$ in $\mathrm{H}_{2} \mathrm{O}$ .126

Figure 6.10 Comparing $\alpha(0)$ values in $\mathrm{H}_{2} \mathrm{O}$ and $\mathrm{D}_{2} \mathrm{O}$

Figure 6.11 Effect of chain length on the standard rate constant in $\mathrm{H}_{2} \mathrm{O}$ 128

Figure 6.12 Generic diagram showing redox molecule on thinner vs thicker chain...129

Figure 6.13 Slopes of the anodic and cathodic branch obtained from the plot of $\alpha(0)$ vs

$\eta$ in $\mathrm{H}_{2} \mathrm{O}$

Figure 6.14 Reorganization energy for $\mathrm{Os}^{\mathrm{II}}$ and Os ${ }^{\mathrm{III}}$ as a function of $\mathrm{pH}$

Figure 6.15 Comparing reorganization energies in $\mathrm{H}_{2} \mathrm{O}$ vs $\mathrm{D}_{2} \mathrm{O}$ 135

Figure 7.1 Square scheme II: Proposed PCET mechanism 


\section{List of Tables}

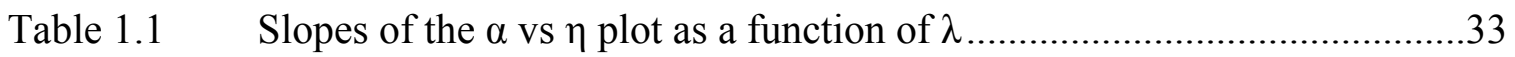

Table 4.1 Experimental and literature UV data for Os(bpy $)_{2} \mathrm{Cl}_{2} \ldots \ldots \ldots \ldots \ldots \ldots \ldots \ldots \ldots . . . .69$

Table 4.2 Formal potentials data for $\left[\mathrm{Os}(\mathrm{bpy})_{2}\left(\mathrm{OH}_{2}\right)_{2}\right]^{2+}$ from $\mathrm{Os}(\mathrm{bpy})_{2} \mathrm{CO}_{3} \ldots \ldots \ldots . . .74$

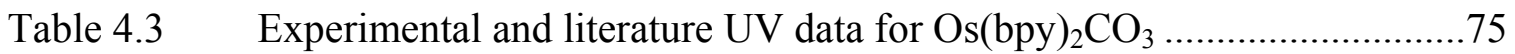

Table 6.1 Thermodynamic parameters for the $\left[\mathrm{Os}(\mathrm{bpy})_{2}(\mathrm{py})\left(\mathrm{OH}_{2}\right)\right]^{2+} \ldots \ldots \ldots \ldots \ldots \ldots . . . .111$ 


\section{Abbreviations}

DoS

density of states

PCET

proton coupled electron transfer

SAM

self-assembled monolayer

FWHM

full width at half maximum peak

CV

Cyclic Voltammetry

UV

Ultraviolet

EDC

1-ethyl-3(3-dimethylaminopropyl)-carbodiimide

DMF

dimethyl formamide

GPIB

General Purpose Instrument Bus

py

4-Aminomethylpyridine

bpy

bipyridine 


\section{SYMBOLS}

$\mathrm{e}^{-}$

$\mathrm{n}$

$\mathrm{h}$

$\mathrm{Ox}$

Red

$\mathrm{k}_{\mathrm{a}}$

$\mathrm{k}_{\mathrm{c}}$

$\mathrm{k}_{\mathrm{s}}$

$v$

i

F

A

$\mathrm{P}$

$\mathrm{R}$

E

$\alpha$

$\lambda$

$\lambda_{\text {is }}$

$\lambda_{\mathrm{os}}$

$\mathrm{D}_{\mathrm{op}}$

$\mathrm{D}_{\mathrm{s}}$ $|V|^{2}$ electron

number of electrons transferred

number of protons transferred

oxidized species

Reduced species

anodic rate constant

cathodic rate constant

standard rate constant

overall reaction rate

current

Faraday's constant

area of electrode

pre-exponential factor

molar gas constant

formal potential

transfer coefficient

reorganization energy

inner sphere reorganization energy

outer sphere reorganization energy

optical dielectric constant

static dielectric constant

electronic coupling parameter 


$\begin{array}{ll}\mathrm{D}(\varepsilon \lambda \eta) & \text { density of redox molecule states } \\ \mathrm{f}(\varepsilon) & \text { probability of occupancy for metal states } \\ \varepsilon & \text { energy level where electron transfer occurs } \\ \Gamma \mathrm{x} & \text { surface coverage of attached species } \mathrm{x} \\ \mathrm{K}_{\mathrm{a}} & \text { acid dissociation constant } \\ \mathrm{k}_{\mathrm{s}, \text { app }} & \text { apparent standard rate constant } \\ \alpha(0) & \text { transfer coefficient at zero overpotential } \\ \eta & \text { overpotential } \\ \mathrm{i}_{\mathrm{ch}} & \text { charging current } \\ \mathrm{Q}_{\text {tot }} & \text { total charge } \\ \mathrm{R}_{\mathrm{u}} & \text { uncompensated resistance } \\ \eta_{0.5} & \text { overpotential at } 50 \% \text { conversion of reactant to product } \\ \mathrm{Q}_{0.5} & \text { the charge transferred between electrode \& redox molecule } \\ \Delta \mathrm{e} & \end{array}$




\section{Extended Abstract}

This work was necessitated by the need to understand the mechanism of protoncoupled electron transfer reactions. These reactions are prevalent in many biochemical systems such photosynthesis and respiration. Current studies on proton-coupled electron transfer reactions focus more on homogeneous systems. This work provides an alternative method by considering a heterogeneous system where a model redox molecule; the Os ${ }^{\mathrm{II}}(\mathrm{bpy})_{2}(\mathrm{py})\left(\mathrm{OH}_{2}\right)$, is attached to an electrode through a self-assembled monolayer. Although many reports have appeared for homogenous studies of protoncoupled electron transfer reactions, not much has been reported using heterogeneous systems. Previous work by Haddox with the same compound was based on a shorter chain self-assembled monolayer.

In this work, a longer chain self-assembled monolayer is used as a bridge between the redox molecule and the electrode. Increasing the length of the self-assembled monolayer reduces the electronic coupling between the redox molecule and the electrode. Consequently, the rates of electron transfer are reduced to values that are measurable using common equipment and Cyclic Voltammetry. In addition to using a longer chain, experiments are repeated in deuterated electrolytes to investigate the kinetic isotope effect. The kinetic isotope effect is the ratio of the standard rate constant calculated in light water to that calculated in heavy water (deuterium oxide). To our knowledge, this is the first study that looks at the isotope effect for a surface confined redox molecule. The effect of chain length on the thermodynamic and kinetic properties of the osmium system is examined in both light and heavy water. Data treatment is based on a potential dependent transfer coefficient as per the Marcus model. 
In Haddox's analysis, the data was interpreted in terms of the stepwise model. This model could not adequately explain the observed kinetics. In this study, the observed kinetics are rationalized by invoking a concerted mechanism. A recent theoretical treatment by Constentin and coworkers ${ }^{14}$ predicts that: (a) the same expression used to describe the standard rate constant in simple electron transfer can be used in PCET reactions; (b) the outer sphere component of the total reorganization energy is made up of two parts; the electron transfer component and the proton transfer component; (c) the pre-exponential factor which incorporates the metal density of states and electronic coupling between the metal and redox molecule can be used to calculate the kinetic isotope effect; and finally; (d) slowness of PCET reactions has been attributed to a small value of the pre-exponential factor rather than due to a large reorganization energy. In this study, analysis and interpretation of data incorporates some of these ideas to investigate if the concerted mechanism can be used to reasonably explain the observed trends in kinetics. The ultimate goal of this study is to fully understand the mechanism of proton coupled electron transfer by investigating thermodynamic and kinetic properties of a model inorganic complex, $\left[\mathrm{Os}(\mathrm{bpy})_{2}(\mathrm{py})\left(\mathrm{OH}_{2}\right)\right]\left(\mathrm{PF}_{6}\right)_{2}$. Data interpretation is accomplished by incorporating the Marcus Density of states theory into a theoretical model developed by E. Laviron.

\section{Organization of this dissertation.}

Chapter one provides a theoretical background of simple electron transfer as it applies to a surface confined redox molecule. Both the Butler-Volmer model and the Marcussian treatments are examined. Theoretical concepts based on different reorganization energies between the oxidized and reduced species are also examined. 
This Chapter provides the background which leads up to more complex situation of a 1e, $1 \mathrm{H}$ system described in the third Chapter.

The second Chapter provides an overview of self-assembled monolayers. This is not an exhaustive review. It simply introduces the concepts that are relevant to this study. For a more comprehensive review about self-assembled monolayers and their application, the reader is referred to a recent excellent review by Christopher Love and coworkers. ${ }^{18}$

Chapter three is a brief introduction to proton-coupled electron transfer reactions with special emphasis to a heterogeneous system. The theoretical background provided in this Chapter is an alternative approach to the homogeneous approach of understanding the mechanism of proton-coupled electron transfer reactions. Most of the derivations are based on work developed by E. Laviron during the late 1970s and early 1980s and are applicable to a redox molecule confined to the surface of an electrode.

The fourth Chapter summarizes all the synthetic work and product confirmation methods required to reach the final product. Most of the synthetic work is based on established methods.

Experimental procedures, instrumentation and data analysis methods are provided in the fifth Chapter.

The sixth Chapter gives the results for the 1e,1H osmium system. Experimental results are also compared and tested against the stepwise model of proton-coupled electron transfer. Deviations from the stepwise model are addressed and rationalized in terms of the concerted model. The kinetic isotope effect and its relevance to the concerted mechanism are also discussed. Reorganization energies are calculated for the oxidized and reduced species. 
The seventh Chapter discusses and explains the observed trends in results. A modified square scheme mechanism is proposed. The results are then summarized and future directions of the project suggested. 


\section{CHAPTER 1: INTRODUCTION TO SIMPLE ELECTRON TRANSFER THEORY: BUTLER-VOLMER MODEL.}

\subsection{Background}

The knowledge gained from understanding the mechanistic pathways and kinetics of electron transfer reactions can find widespread applications in many chemical (electrophoresis, corrosion, electroanalytical sensors and fuel cells) and biological (photosynthesis and enzymic processes) ${ }^{1}$ systems. It is important to have a fundamental understanding of electron transfer processes in order to understand these processes to a deeper level. To achieve this, it is imperative to have a simple model as a starting point.

Since electron transfer at electrode surfaces has similar characteristics to that in homogeneous solutions, electrochemical techniques can be utilized to characterize electron transfer reactions. One way to do this is to look at electrochemical processes where charges (electrons) are transferred across a metal-solution interface ${ }^{1}$. These types of reactions cause oxidation or reduction to occur on the surface of the electrode. Such redox reactions can cause current to flow, and this current can be monitored as a function of potential to provide thermodynamic and kinetic information for a given system.

Two types of charge transfer occur at electrodes. Faradaic processes are caused by oxidation or reduction of analyte species on the surface of the electrode. In these processes, the amount of charge passed due to flow of electrons is proportional to the concentration of reactants in solution. Other processes such as adsorption and desorption of species on the electrode surface can also occur, leading to changes in the electrodesolution interface structure with changes in potential or solution composition. Such changes in potential can lead to a transient flow of current called non-faradaic or charging 
current $^{1}$. This current has nothing to do with redox processes of the analyte. With current techniques such as cyclic voltammetry, however, the charging current can be easily subtracted to obtain the useful faradaic current.

Despite the significance of electron transfer reactions in our daily lives, their study has been a challenging task. The challenges stem from several reasons. First, the rates of electron transfer can be so high that special techniques are required to measure them. Recently, Scanning Electrochemical Microscopy (SECM) has been applied to study very fast electron transfer using a microelectrode. ${ }^{2}$ However, SECM is an expensive and specialized technique.

The second challenge in studying rates of electron transfer in homogeneous solution emanates from the fact that the rates of electron transfer can be affected by double layer effects. Double layer effects arise from two sources. First, the electrode/electrolyte interface exhibits a capacitance whose magnitude reflects the distribution of ions on the solution side of the interface. The Helmholtz layer consists of a layer of ions and solvent in physical contact with the electrode and the diffuse layer is a layer of ions near the electrode whose concentration deviates from the bulk concentration. When a potential is applied on a working electrode, it creates a charge on the electrode. This charge can cause redox ions to be attracted to the electrode or repelled by the electrode depending on their relative charges. The charged electrode and the layer of oppositely charged ions next to the electrode make up an electric double layer. ${ }^{3}$ As a result of this, the concentration of redox ions on the surface of the electrode can be significantly different from the bulk concentration. In order to convert current to rate 
constants, the concentration of redox molecules on the surface of the electrode must be known.

The second source of double layer effects is more subtle. A redox ion inside the diffuse layer or inner Helmholtz layer can experience a different potential than an ion in the bulk solution. As the electrode potential changes, the potential experienced by the redox ion near the electrode is different from the potential experienced by the other ions in solution. For example, a $0.1 \mathrm{~V}$ change in applied potential could cause a smaller change in the potential difference between the redox ion in the Helmholtz layer and the electrode. Consequently, the influence of the double layer on electron transfer is complex and corrections of rate constants for double layer effects are difficult. ${ }^{4}$

Finally, electron transfer rates can be limited by the rate at which the redox molecule is transported to the electrode through processes such as diffusion, convection and migration, especially at a large driving force. All these problems make the study of electron transfer kinetics difficult, especially with currently available techniques.

The advent of chemically modified electrodes, where the redox molecule is attached to the surface of the electrode, has benefited the area of electron transfer studies greatly. In particular, through the use of surface modification techniques such as Selfassembled Monolayers (SAMs), the redox molecule can be immobilized on the surface of the electrode without losing any of the chemical information obtained on a bare electrode. ${ }^{4}$

The benefits of using Self-assembled Monolayers include the fact that mass transfer and double layer effects are eliminated. Since the redox molecule is attached to the electrode and there are no redox species in the bulk solution, the rate is purely 
dependent on electron transfer and not mass transfer. Also, since the Helmholtz layer (ions and solvent molecules specifically adsorbed to electrode surface) has been replaced by a hydrocarbon layer of low dielectric constant, double layer effects are eliminated. Finally, bridging the redox molecule and the electrode with a hydrocarbon layer means that electron transfer between the redox molecule and the electrode has to occur through tunneling. Since tunneling is a low probability process, the rate is reduced to values that are measurable with common techniques such as Cyclic Voltammetry. More details about Self-assembled Monolayers will be given in Chapter 2. The discussion from hereon will focus on electron transfer as it applies to surface modified electrodes.

One of the first successful theoretical formulations for electrode reactions was developed by John Alfred Valentine Butler and later modified by Max Volmer to give what is popularly known as the Butler-Volmer Equation. This Equation has had some profound effects on electrochemistry and in particular, in the evaluation of thermodynamic and kinetic properties of heterogeneous reactions. The following section will provide a closer look at the Butler-Volmer Equation and its consequences.

Consider a generalized simple one electron half reaction:

$\mathrm{Ox}+\mathrm{e}^{-} \Leftrightarrow \operatorname{Red}$

The overall rate of electron transfer $(v)$, in moles $/ \mathrm{s} / \mathrm{cm}^{2}$ is related to net current, $i=i_{a}-i_{c}$ ( $i_{a}$ is the anodic current, $i_{c}$ is the cathodic current), the area of the electrode $(\mathrm{A})$ and the surface concentrations of redox species by the Equation:

$v=\frac{i}{F A}=k_{a} \Gamma_{\operatorname{Red}}-k_{c} \Gamma_{o x}$

$\Gamma_{\text {Red }}$ is the surface concentration $\left(\right.$ moles $/ \mathrm{cm}^{2}$ ) of reduced species, $\Gamma_{\mathrm{Ox}}$ is the surface concentration of the oxidized species, $k_{a}$ is the anodic rate constant and $k_{\mathrm{c}}$ is the cathodic 
rate constant. It is important to note that two conventions are often used in electrochemical literature to define the sign of the current for anodic and cathodic processes. In the American convention, anodic processes produce negative currents while cathodic processes yield positive currents. The IUPAC convention is more widely used and defines the anodic current as positive and cathodic current as negative. The IUPAC convention will be used here for all definitions of anodic and cathodic processes.

The rate constants can be represented by a general expression of the Arrhenius Equation:

$k=A e^{-E_{a} / R T}$

$E_{a}$ is the activation energy (thermal energy is required to overcome an energy barrier of height equal to $E_{a}$ ). $A$ is the frequency factor (the number of attempts on climbing the energy barrier). $\mathrm{T}$ is the absolute temperature $(\mathrm{K})$ and $\mathrm{R}$ is the molar gas constant $(\mathrm{J} / \mathrm{mol} / \mathrm{K})$. The reaction pathway or coordinate can be plotted as a function of energy, (Figure 1.1). 


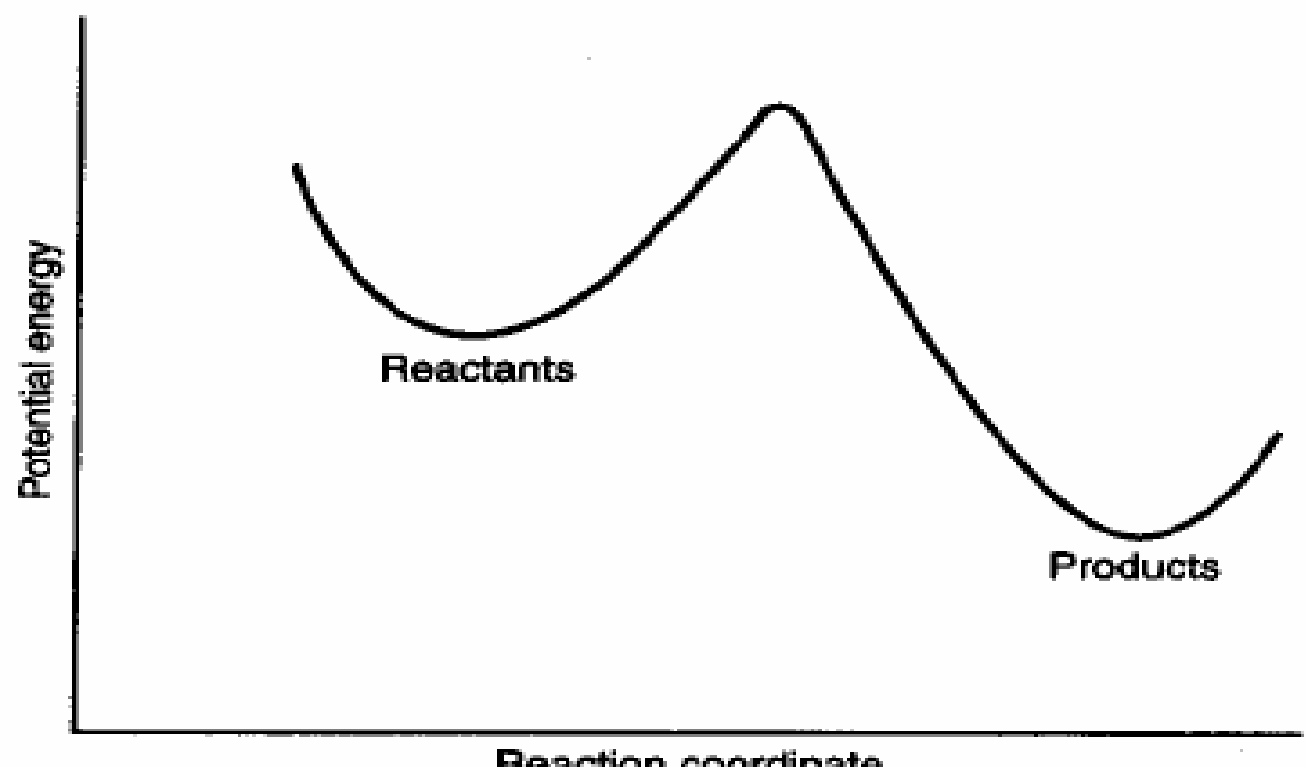

Reaction coordinate

Figure 1.1. Changes in potential energy during a redox reaction at an electrode. Diagram taken from Bard and Faulkner'.

In the above diagram, the reaction pathway connects two minima. As the reaction proceeds, the reactant coordinates are changed to those of the product. The reactant passes through an energy maximum during this transition to the product. This maximum defines the energy barrier height or activation energy ${ }^{1}$. Equation (1.3) can be rewritten as:

$k=A e^{-\Delta G^{*} / R T}$

where $\Delta G^{\neq}$is the standard free energy of activation. 


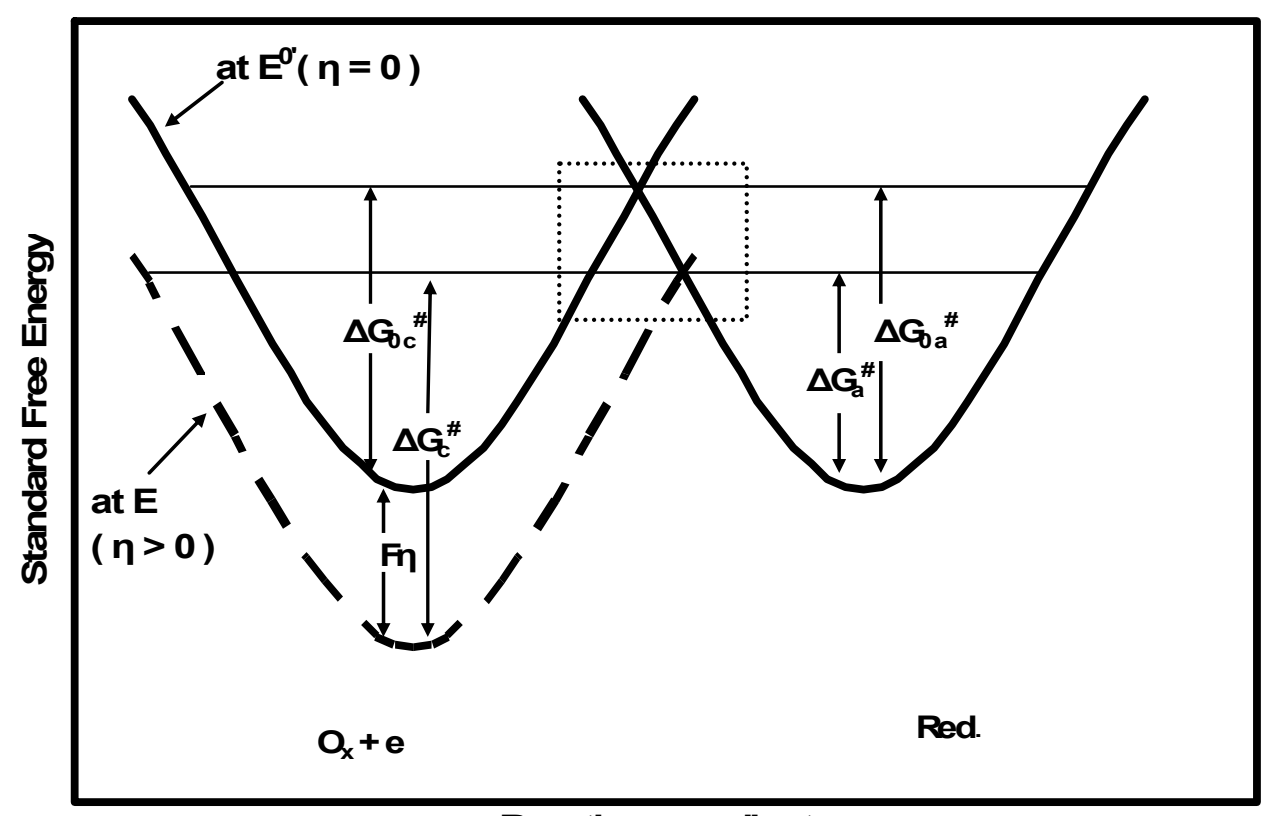

Reaction coordinate

Figure 1.2: Effects of change in potential on the energy barrier for a simple redox process. This Figure was adapted from Bard and Faulkner. ${ }^{1}$

If the two minima have the same height as shown in Figure 1.2 (solid curves), then $\Delta \mathrm{G}=0, i=0$ and from Equation 1.2, $\mathrm{k}_{\mathrm{a}} \Gamma_{\mathrm{Red}}=\mathrm{k}_{\mathrm{c}} \Gamma_{\mathrm{Ox}}$. The formal potential is defined under these conditions. The formal potential will be used as a reference point for all kinetic expressions in this discussion. Any shift in potential will therefore be defined relative to the formal potential.

In Figure 1.2, the upper curve on the $\mathrm{Ox}+\mathrm{e}^{-}$side is defined when the electrode potential is equal to the formal potential, $\mathrm{E}^{0^{\prime}}$. Under such conditions, the cathodic and anodic rate activation energies are represented by $\Delta G_{0 c}^{\neq}$and $\Delta G_{0 a}^{\neq}$respectively. Any perturbation of the system at the formal potential shifts the potential to a new value. The difference between the new potential and the formal potential $\left(E-E^{0^{\prime}}\right)$ is the 
overpotential $(\eta)$ or the driving force for electron transfer. The free energy curve shifts either up or down by an amount corresponding to the applied potential. If a positive potential is applied, the free energy of the electron decreases and the curve shifts down by

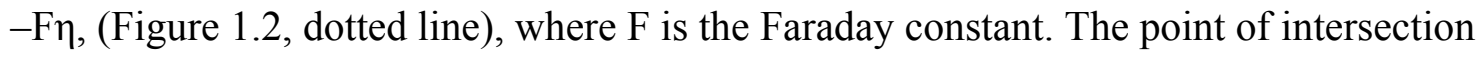
for the two curves defines the transition state; the height of the barriers to oxidation or reduction is related to their relative rates of electron transfer ${ }^{1}$. The consequence of shifting the curve downwards is that the energy barrier for oxidation, $\Delta G_{a}^{\neq}$, decreases relative to $\Delta G_{0 a}^{\neq}$, by some fraction (1- $\left.\alpha\right)$ of the total free energy change. Alpha is known as the transfer coefficient; details about this parameter will be given in later sections. Values of $\alpha$ range from zero to unity depending on the shape of the intersection region. If the fraction for oxidation is defined by $(1-\alpha)$, then, the free energy of oxidation is given by:

$$
\Delta G_{a}^{\neq}=\Delta G_{0 a}^{\neq}-(1-\alpha) F \eta
$$

Likewise, the barrier for reduction, $\Delta G_{c}^{\neq}$, has increased, relative to $\Delta G_{0 c}^{\neq}$, leading to the Equation:

$$
\Delta G_{c}^{\neq}=\Delta G_{0 c}^{\neq}+\alpha F \eta
$$

Figure 1.3 is a closer look at the intersection region enclosed by the dotted box in Figure 1.2 , and shows the relative fractions by which the anodic and cathodic energy barriers change as a result of a positive applied potential. 


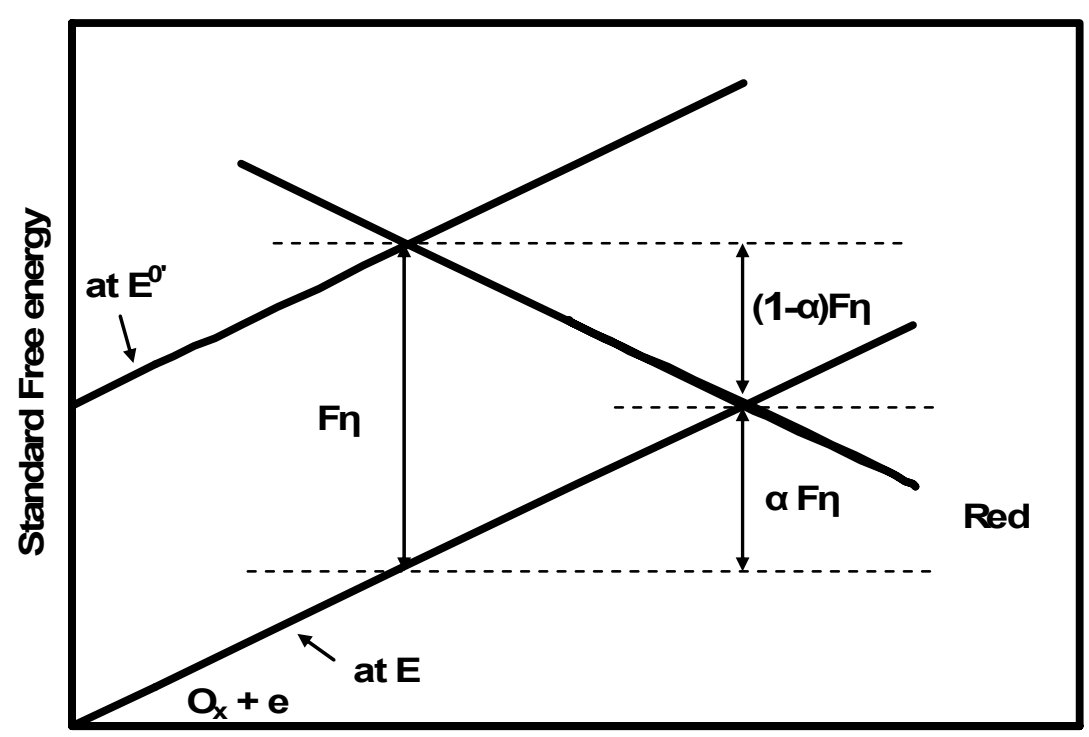

Reaction Coordinate

Figure 1.3. An expanded view of the intersection region shown by the dotted box in Figure 1.2.

From Equation 1.4, the anodic and cathodic rate constants ( $\mathrm{k}_{\mathrm{a}}$ and $\mathrm{k}_{\mathrm{c}}$ respectively), can be obtained by substituting $\Delta G^{\neq}$with the respective expressions for the free energy (Equation 1.5 and 1.6), thus:

$k_{a}=A_{a} e^{-\left(\frac{\Delta G_{a}^{ \pm}}{R T}\right)}=A_{a} e^{-\left(\frac{\Delta G_{0 a}^{ \pm}}{R T}\right)} \cdot e^{\frac{(1-\alpha) F \eta}{R T}}$

$k_{c}=A_{c} e^{-\left(\frac{\Delta G_{0 c}^{ \pm}}{R T}\right)}=A_{c} e^{-\left(\frac{\Delta G_{0 c}}{R T}\right)} \cdot e^{-\frac{\alpha F \eta}{R T}}$

For a special case where the system is at the formal potential and $\Gamma_{O x}=\Gamma_{\operatorname{Red}}$, it can be shown from Equations 1.7 and 1.8 that:

$k_{s}=A_{a} e^{-\frac{\left(\Delta G_{0 . a}^{ \pm}\right)}{R T}}=A_{c} e^{-\frac{\left(\Delta G_{0 . c}^{ \pm}\right)}{R T}}$ 
where $k_{s}$ is standard rate constant, defined at the formal potential (zero driving force $(\eta=$ $0)$ ). The first two terms of each of Equation 1.7 and 1.8 constitute a product that is independent of potential, but equal to the standard rate constant $\mathrm{k}_{\mathrm{s}}{ }^{1}$ Substituting $\mathrm{k}_{\mathrm{s}}$ into Equation 1.7 and 1.8, the following Equations can be obtained:

$k_{c}=k_{s} e^{-\alpha f \eta}$

$k_{a}=k_{s} e^{(1-\alpha) f \eta}$

where: $f=\frac{F}{R T}=38.92 V^{-1}$ at $25^{\circ} \mathrm{C}$

The exponential dependence of the rate constant on overpotential means that any small change in overpotential could have significant consequences on the rate constant.

Substituting Equation 1.10 and 1.11 into Equation 1.2 yields the general current-voltage Equation-the Butler-Volmer Equation:

$i=F A k_{s}\left(\Gamma_{\operatorname{Red}} e^{(1-\alpha) f \eta}-\Gamma_{O X} e^{-\alpha f \eta}\right)$

This Equation is one of the first successful formulations to qualitatively describe how kinetically controlled current changes with driving force and has been the workhorse for electron transfer kinetic for decades.

The cathodic rate constant (Equation 1.10) can be re-written in logarithmic form:

$\log k=\log k_{s}-\frac{\alpha F}{2.3 R T}$

Similarly the anodic rate constant (Equation 1.11) can be represented as:

$\log k=\log k_{s}+\frac{(1-\alpha) F \eta}{2.3 R T}$

Both expressions can be generalized as:

$\log k=a+b \eta$ 
where $a=\log k_{s}$ and $b=\frac{(1-\alpha) F}{2.3 R T}$ for the anodic rate constant and $b=\frac{-\alpha F}{2.3 R T}$ for the cathodic rate constant. Equation 1.15 is known as the Tafel Equation, named after its discoverer in $1905^{1}$.

Kinetically controlled reactions have been shown to obey the Tafel Equation. A plot of $\log (\mathrm{k})$ versus $\eta$ is known as the Tafel plot (Figure 1.4). Such a plot provides a convenient way of evaluating kinetic parameters. Provided there is no curvature in the Tafel region, the transfer coefficient can be obtained from the slope of each curve, which is the b-term of Equation 1.15; the a-term is the intercept. If $\alpha=0.5$ and independent of potential, Tafel plots should be linear and symmetrical and the slope for the anodic branch will be the same as that for the cathodic branch.

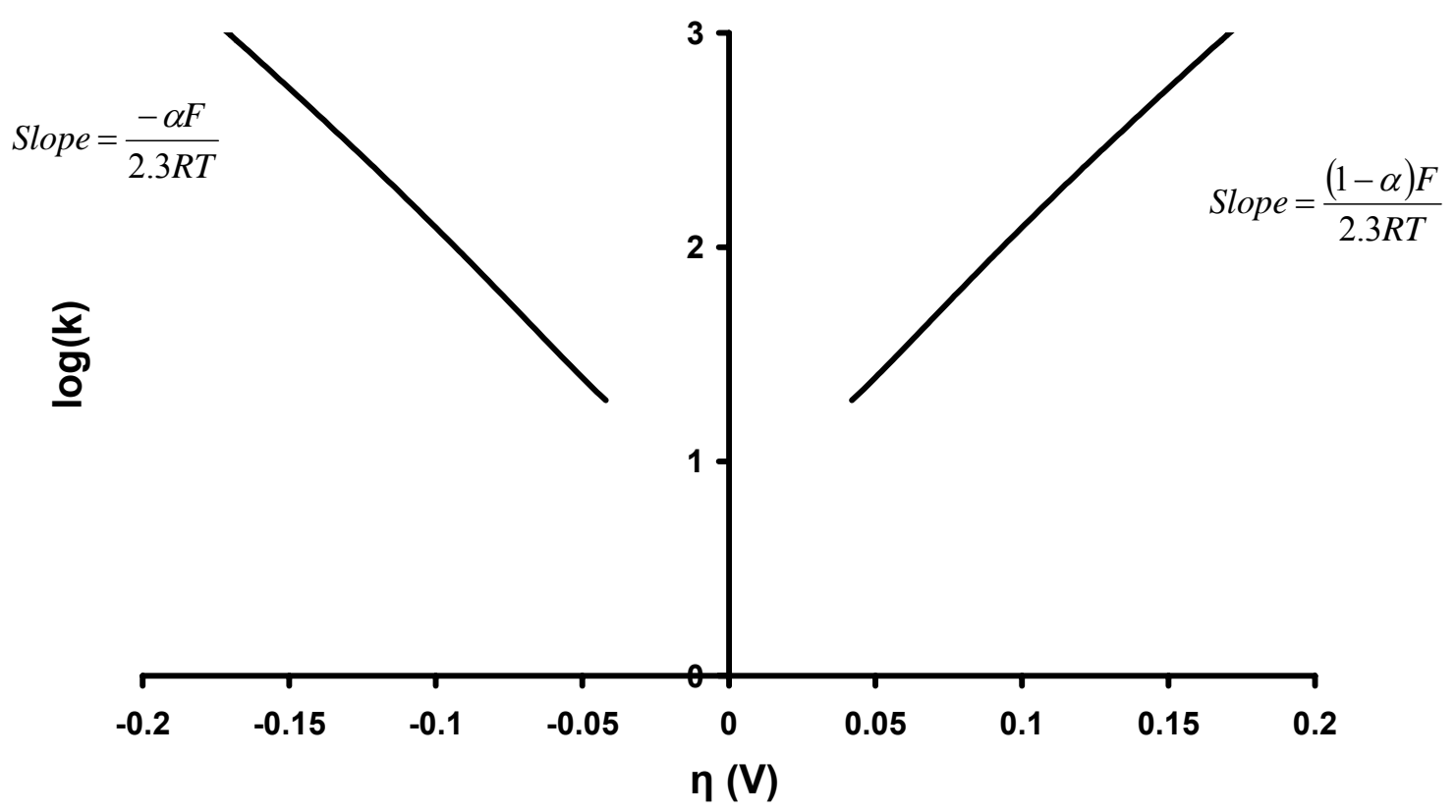

Figure 1.4. Typical Tafel plot: $\log (\mathrm{k})$ vs $\eta$ 
However it is important to note that, as shall be seen later, if the transfer coefficient is potential dependent, then the Tafel plots can be asymmetrical.

\subsection{Limitations of the Butler-Volmer Theory.}

Although the Butler-Volmer Equation has been widely used to provide information about reaction mechanisms ${ }^{1}$ as well as to relate the apparent rate of electron transfer to the standard rate constant and the applied driving force, it is based on classical (macroscopic) concepts. The model presents a picture that is too broad and too simplistic to fully describe the reality of redox systems. It ignores any contributions to the apparent rate of electron transfer from such factors as the nature, orientation and structure of reactants near the electrode surface, electrode material, position and orientation of solvent molecules ${ }^{2}$, the effect of adsorbed layers ${ }^{1}$ or thin films on the electrode. As a result of these limitations, it was necessary to develop a much more comprehensive theoretical treatment that looks at microscopic events occurring at or near the electrode surface. The currently accepted theoretical treatment of electron transfer on a microscopic level is based on an analysis developed by Rudolph A. Marcus in the 1950s and 1960s ${ }^{2,3}$, and popularly known as the Marcus Density of States (DoS) theory. More details about this theoretical analysis is given in later sections.

\subsection{Physical Meaning of the Standard Rate Constant.}

The standard rate constant is the intrinsic rate at which the oxidized species and the reduced species exchange electrons with the electrode at the formal potential. It is a measure of how kinetically facile a redox couple is. A system that has a large value of $\mathrm{k}_{\mathrm{s}}$ reaches equilibrium faster than a system with a small value of $\mathrm{k}_{\mathrm{s}}$. For surface attached

redox species, standard rate constants as high as $10^{7} \mathrm{~s}^{-1}$ have been reported. ${ }^{5,6}$ Even in 
cases where $\mathrm{k}_{\mathrm{s}}$ is very small, the values for anodic and cathodic rate constants can be increased dramatically by applying a large potential to supply the activation energy in the form of electrical energy. ${ }^{1}$

\subsection{The transfer coefficient}

The transfer coefficient $\alpha$ is a parameter that shows how the total free energy of activation for electron transfer is divided between the anodic and cathodic steps. It can also be used to assess the symmetry of the energy barrier ${ }^{1}$. In Figure 1.1, the assumption is that the two curves are symmetrical and have the same curvature. When the system is at the formal potential, the point of intersection ( activated complex) lies midway between the minimum for each curve. In this case, $\alpha$ is equal to 0.5 . However, if the left curve is lowered relative to the right curve (by applying a positive potential), the point of intersection lie closer to the Red species than the Ox species. Alpha is greater than half and the barrier for oxidation is lower than that for reduction. ${ }^{1}$ Conversely, if the left curve is raised ( by applying a negative potential), the activated complex lies closer to the Ox species than the Red species. Alpha is less than half and the barrier to reduction is lower

than that for oxidation. ${ }^{1}$ Both these cases lead to asymmetry in Tafel plots as shall be seen later.

Since the point of intersection changes and the geometry of the point of intersection changes with potential, the transfer coefficient tends to be potential dependent. If the free energy curves are parabolas with equal curvature, as shown in Figure 1.2, the transfer coefficient varies linearly with overpotential, Figure 1.5. ${ }^{1}$ 


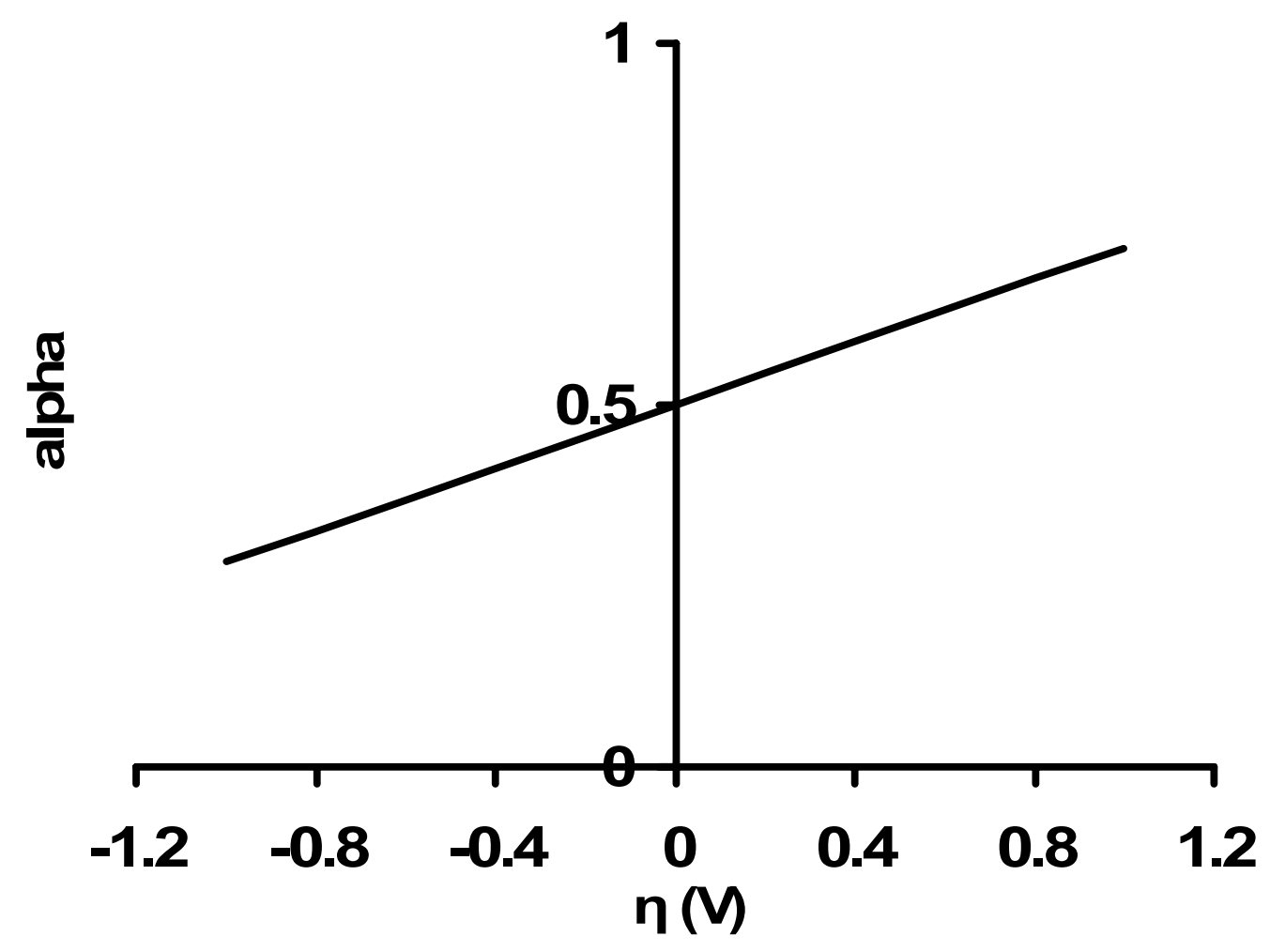

Figure 1.5.Potential dependence of transfer coefficient as predicted by the parabolic free energy curves with equal curvature.

The line has a constant slope for both the anodic and cathodic branch. Also, the plot yields an alpha( 0$)$ of 0.5 at zero overpotential. In the next section, the curvature of the parabolas will be related to the reorganization energies of the oxidized and reduced species. As shall be seen later, if the reorganization energies for the Ox and Red species are different, plots of $\alpha$ versus $\eta$ often exhibit different slopes between the anodic and cathodic branch. 


\subsection{Marcus Density of States Model: A closer look.}

Marcus DoS theory is an extension of the Butler-Volmer model and considers events that occur on a microscopic level. Therefore, electron transfer rate is described based on quantum mechanical concepts rather than the classical approach used in the Butler-Volmer model. $^{7}$ There are two alternatives to the Marcus treatment of heterogeneous electron transfer. The first alternative is based on the transition state

approach. ${ }^{1,8}$ In this approach, electron transfer is assumed to occur either via the outersphere mechanism or inner sphere mechanism. This terminology was borrowed from that used in the chemistry of coordination compounds ${ }^{1}$. The inner-sphere mechanism is more applicable to electron transfer in solution and will not be discussed here. Also, because of its complexities, the inner-sphere mechanism has not been as theoretically developed as the outer-sphere mechanism.

Because of its simplicity, the outer-sphere mechanism has been much more developed than the inner-sphere mechanism and will be the subject of the following discussion. The outer-sphere mechanism occurs when there is weak coupling between the redox molecule and the electrode. This weak interaction emanates from the fact that the redox molecule is generally at a distance of at least a solvent molecule away from the electrode. In the outer-sphere mechanism, the original coordination spheres are preserved in the activated complex.

The electron transfer step is considered to be the rate determining step and the electron transfer process is considered to involve several steps prior to the actual transfer of the electron. Although this assumption is an arbitrary formalism, it simplifies the 
chemistry involved and provides simple concepts on which to build a mathematical model.

First, thermal energy is required to activate the redox molecule. This energy sets the redox molecule into random thermal motion that alters the structure and solvation sphere of the redox molecule. The donor and acceptor levels of the redox molecule oscillate about an equilibrium energy corresponding to the most stable molecular and solvent structure of the redox center. Through these random fluctuations, the redox molecule is brought into resonance with appropriate electronic states in the electrode. At this point, electron transfer occurs through tunneling between the electrode and the redox center. The probability of electron exchange between donor and acceptor orbitals can vary from zero to unity ${ }^{8}$ depending upon several factors such as orientation of electrode surface, distance between participating orbitals, the density of electronic states in the electrode as well as the structure of the spacer between the redox molecule and the electrode. The actual electron transfer step is a radiationless step and the redox molecule changes from one oxidation state to the other without changes in its internal and solvent structure. In other words, electron transfer occurs between states of the same energy. After electron transfer, the redox molecule relaxes to its ground state geometry. The actual electron transfer step is so fast that momenta and nuclear positions of the redox molecule remain effectively frozen during the timescale of the electronic transition ${ }^{1}$. In this case, the electron transfer step is a distinct step that occurs between activation and relaxation. This is a direct application of the Franck-Condon principle. Any mathematical model that describes the rate constant must incorporate contributions from all the individual elementary steps described here. 
An alternative to the transition state approach is the Marcus theory which is based on the overlap of redox molecule electronic states with those of the metal electrode. The key feature to this model is that electron transfer is an adiabatic process; that is, it occurs without gain or loss of energy. According to this model, redox reactions occur due to overlap between acceptor electronic states and donor states of the electrode and the redox molecule. For reduction to occur, there must be an electron at energy $\varepsilon$ associated with filled metal (electrode) states. The redox molecule must have available acceptor states at the same energy level. In this case, the redox center should be the oxidized species and electron transfer will occur from the filled metal states to the empty redox acceptor states. Likewise, for oxidation, we need empty metal electronic states at energy $\varepsilon$ and occupied redox center states at the same energy. The redox center should be associated with the reduced species and electron transfer will occur from the redox molecule to the electrode.

The band theory in metals describes the electronic states of metals. A metal state diagram consists of bands of energy states whose origin can be traced to atomic orbitals. Atomic orbitals overlap to form molecular orbitals which in turn overlap to form energy bands. These energy bands are so close together that they form a continuum of electronic states which is filled to the Fermi level. The highest occupied band is called the conduction band and has a characteristic density of states rho $(\rho)$ which is a function of energy. Different metals have different density of states, for example; the conduction band of platinum is a mixture of states obtained from "s" and " $\mathrm{d}$ " orbitals on platinum, while that for gold and silver is a mixture of "s" orbitals only. As a result, the density of states for platinum is roughly an order of magnitude higher than for gold and silver. 
The Fermi level $\varepsilon_{\mathrm{f}}$, is the energy level at which there is a fifty percent chance of an energy state being occupied by an electron. To a first approximation, energies below the Fermi level are occupied while those above are empty. A more accurate description of the Fermi energy is given by the Fermi distribution function which describes how the probability of a state being occupied varies with energy. The Fermi function can be defined by the following Equation: ${ }^{9}$

$$
n(\varepsilon)=\frac{1}{\left(1+e^{\frac{\left(\varepsilon-\varepsilon_{f}\right)}{k_{B} T}}\right)}
$$

where $\mathrm{n}(\varepsilon)$ is the fraction of occupied states, $\mathrm{k}_{\mathrm{B}}$ is the Boltzmann constant, and $\mathrm{T}$ is the absolute temperature.

This function can be graphed to show occupied states as a function of energy, Figure 1.6a. The product $\rho . n(\varepsilon)$ gives the density of occupied states as a function of energy and affects the rate of reduction. The density of unoccupied states is defined by the product of the complementary function $(1-\mathrm{n}(\varepsilon))$ and $\rho$ and this affects the rate of oxidation.

For the redox molecule, the density of states are defined by a Gaussian distribution function for each of the respective oxidation states, Ox and Red. These Gaussian distributions are dependent upon a parameter known as the reorganization energy $\lambda$. This is the energy required to convert the molecule and its solvation sphere from the equilibrium structure of one oxidation state to the equilibrium structure of the other without moving the electron in or out of the molecule. Each oxidation state has a reorganization energy associated with it. It is important to note here that this discussion assumes the same reorganization between the oxidized and reduced species. 
The reorganization energy can be thought of as a form of activation energy since the two energies are similar $\left(E_{a} \approx \frac{\lambda}{4}\right)$. There are two components to the reorganization energy, the inner-sphere component as well as the outer-sphere component. Details about this will be given in later sections.

At any given energy, the probability of electron transfer is defined by the product of three functions $\rho, \mathrm{n}(\varepsilon)$, and $\mathrm{D}(\varepsilon, \lambda, \eta)$ at that particular energy. The rate constant is then proportional to the integrated overlap of the three functions over all energy. ${ }^{10}$

$$
\begin{aligned}
& k=\frac{(2 \pi)}{\hbar}|V|^{2} \int D(\varepsilon, \lambda, \eta) f(\varepsilon) d \varepsilon \\
& D(\varepsilon, \lambda, \eta)=\frac{1}{\left(4 \pi k_{B} T\right)^{\frac{1}{2}}} e^{-\frac{(\varepsilon-\lambda-e \eta)^{2}}{4 \pi k_{B} T}}
\end{aligned}
$$

$$
D(\varepsilon, \lambda, \eta)=\frac{1}{\left(4 \pi \lambda k_{B} T\right)^{\frac{1}{2}}} e^{-\frac{(\varepsilon+\lambda-e \eta)^{2}}{4 \pi k_{B} T}}
$$

$$
\begin{aligned}
& f(\varepsilon)=\frac{1}{\left(1+e^{\frac{\varepsilon}{k_{B} T}}\right)} \\
& f(\varepsilon)=\frac{e^{\frac{\varepsilon}{k_{B} T}}}{\left(1+e^{\frac{\varepsilon}{k_{B} T}}\right)}
\end{aligned}
$$

(anodic)

where $\mathrm{D}(\varepsilon, \lambda, \eta)$ is the Gaussian distribution of density of acceptor or donor states of the redox couple at the electrode surface, $f(\varepsilon)=\rho . n(\varepsilon)$ is the density of donor or acceptor states in the metal electrode, $|V|^{2}$ is the electronic coupling factor (assumed to be 
independent of energy), $\varepsilon$ is the energy at which the electron is transferred relative to the Fermi level of the electrode, $\lambda$ is the reorganization energy of the redox molecule. The integral in Equation 1.17 has no analytical solution; however, it can be easily evaluated by numerical methods such as the trapezoidal rule. ${ }^{11}$

As shown in Figure 1.6a, the Gaussian distribution for the oxidized species has a maximum energy of a magnitude of $\lambda_{\mathrm{Ox}}$ above the energy associated with the formal potential of the redox molecule. The peak half width of this Gaussian curve is also proportional to $\lambda_{\text {Ox. }}$. Likewise, the reduced species (Red.) have a Gaussian distribution that is $\lambda_{\text {Red }}$ below the energy associated with the formal potential (not shown). 


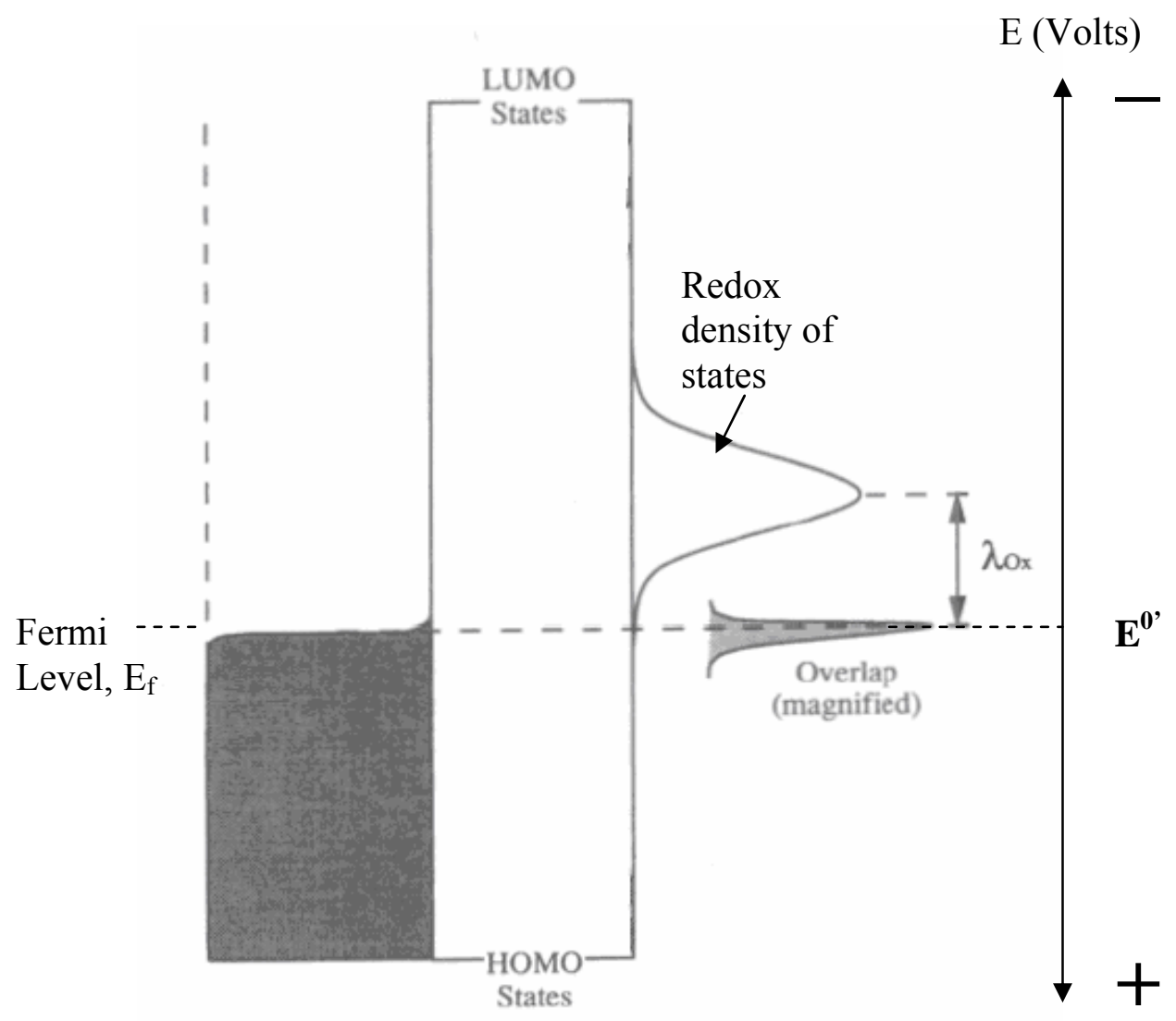

$\begin{aligned} \text { Metal } & \text { Redox } \\ \text { (a) } & \text { molecule states }\end{aligned}$

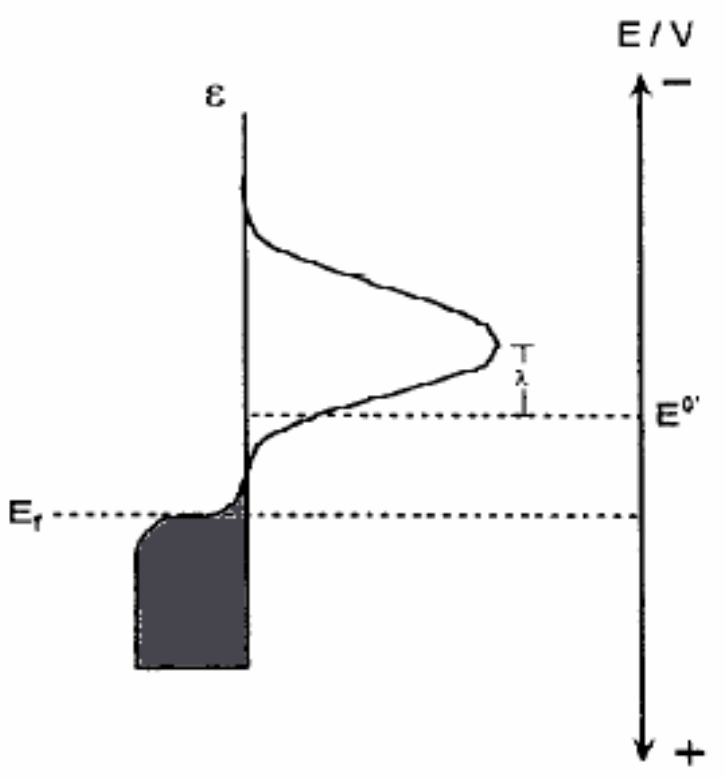

b 


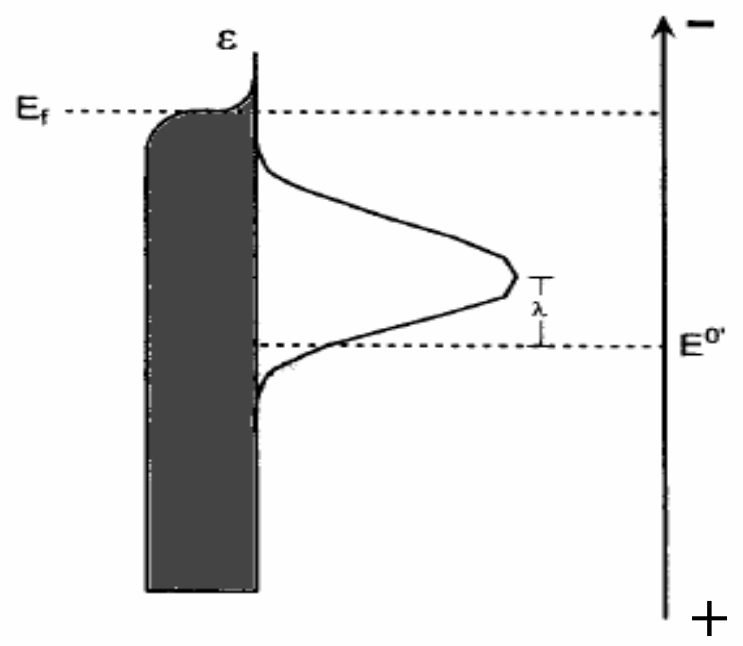

c.

Figure 1.6. Density of states diagram ${ }^{4,12}$ showing the Gaussian distribution of redox species $(\mathrm{Ox})$ and the Fermi distribution function. (a). working electrode polarized at the formal potential of the redox couple (notice the slight overlap between donor and acceptor states even at the formal potential), (b). working electrode polarized positive of formal potential, (c). working electrode polarized negative of formal potential. Figure 1.6 (a) was taken from reference number 4 and Figure 1.6 (b) \& (c) were adapted from reference number 12.

The Fermi energy is the reference point for the energy scale. It is defined relative to the energy of a free electron in a vacuum. If the electrode is polarized by applying either a positive or negative potential, the Fermi level is displaced down or up by an amount proportional to the applied potential. Applying a positive potential, for example displaces the Fermi level downwards because an electron would require more energy to be moved to a vacuum. As shown in Figure 1.6 (b), the filled metal states are displaced 
downwards. The overlap between the metal donor states and Ox acceptor states decreases, so the cathodic rate gets smaller.

Similarly, when a negative potential is applied, the Fermi level is displaced upwards and the overlap increases between filled metal states and the empty redox molecule states. The cathodic rate constant $\mathrm{k}_{\mathrm{c}}$ is proportional to the extent of overlap. At large overpotentials, there is complete overlap (Figure 1.6c) and any further increase in potential has no effect on the rate constant. The rate constant becomes independent of potential (Figure 1.7).

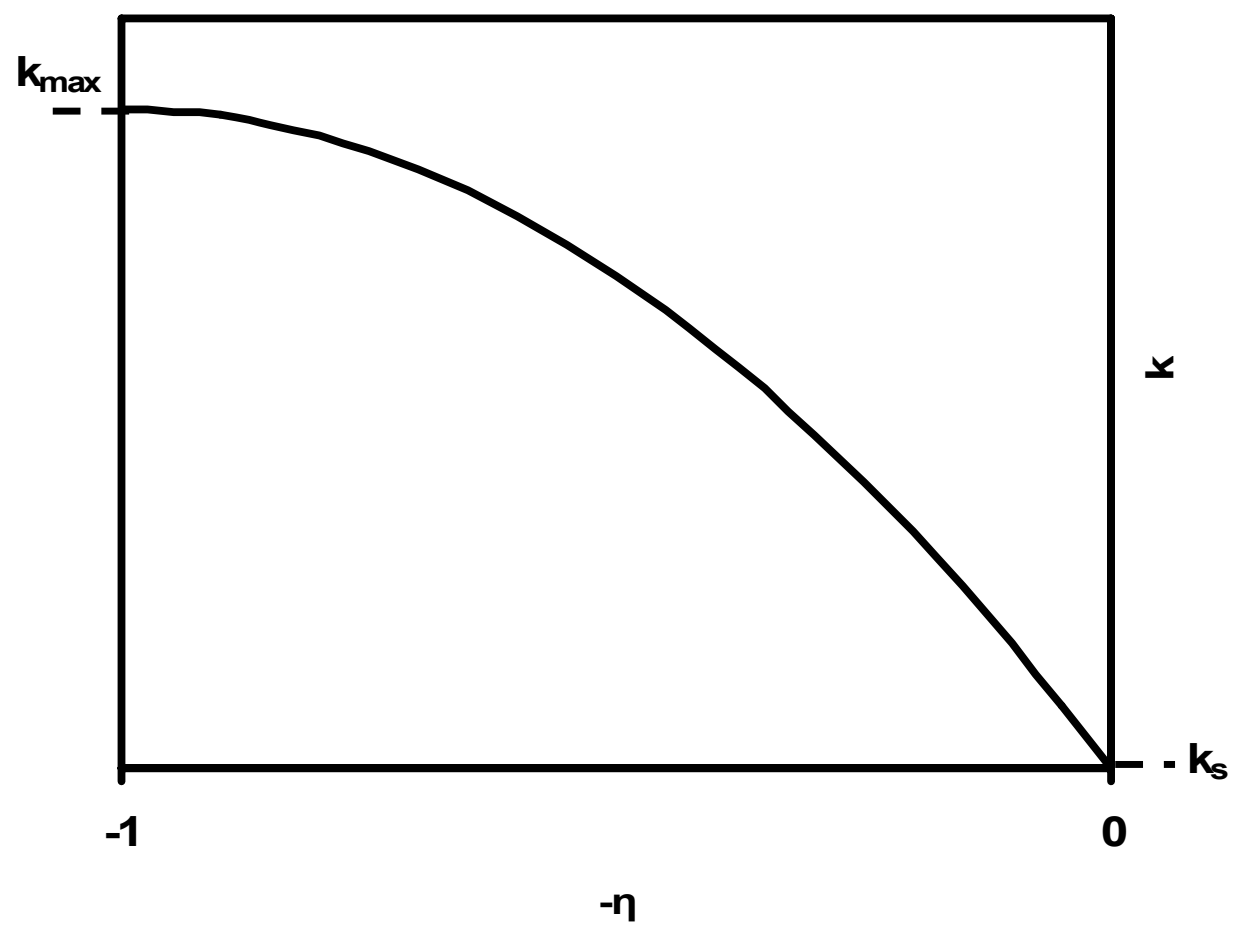

Figure 1.7. Dependence of cathodic rate constant on overpotential. Notice the saturation at large negative potentials. The rate constant reaches a limiting value, $\mathrm{k}_{\max }$. The standard rate constant, $\mathrm{k}_{\mathrm{s}}$, is defined at zero overpotential. A similar analysis can be done for the anodic rate constant. 
The standard rate constant is defined when the electrode potential is set at the formal potential as shown in Figure 1.6a. At this point, the standard rate constant is proportional to the integral defined by Equation 1.17. The standard rate constant has an exponential dependence on the reorganization energy $\left(k_{s} \propto e^{-\left(\frac{\lambda}{4 R T}\right)}\right)$; therefore, it is very sensitive to changes in the reorganization energy.

\subsection{Predictions using Marcus DoS Theory}

One of the most profound consequences of the Marcus model is the prediction of curvature in Tafel plots. As described in the previous section, the overlap integral or rate constants become independent of potential at extreme overpotentials. When this overlap integral is plotted as $\log \left(\mathrm{k}_{\mathrm{s}}\right)$ vs. $\eta$, the resulting Tafel plots show an initial increase with overpotential, followed by a plateau at large overpotentials (e $<<\lambda$, for cathodic branch and en $>>\lambda$, for anodic branch). According to the Butler-Volmer model, there is an indefinitely linear dependence between $\log \mathrm{k}$ and $\eta$, that is, Tafel plots should be indefinitely linear (Figure 1.4).

The degree of curvature is inversely proportional to the reorganization energy.

Figure 1.8 compares the behavior of Tafel plots as a function of reorganization energy. For small $\lambda$, for example, there is noticeable curvature even at small overpotentials. For large $\lambda$ values, however, the plots are linear at small overpotential. In this region, Marcus theory agrees with the Butler-Volmer treatment. The plot for $\alpha=0.5$ is shown for comparison between the Butler-Volmer theory and Marcus Theory. 


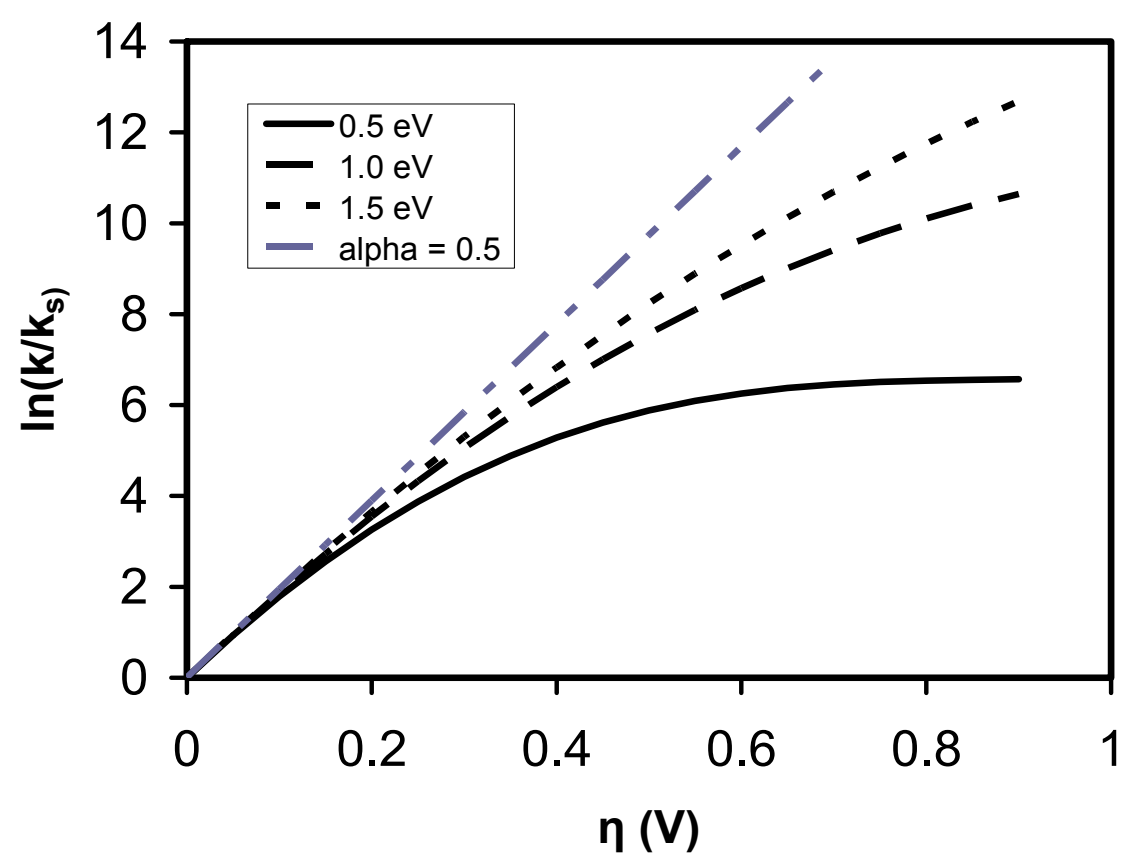

Figure 1.8. Theoretical Tafel plots at various reorganization energies. The rate constants are normalized with respect to the standard rate constant at each reorganization energy.

Another useful piece of information obtained from the Marcus DoS treatment is the dependence of the transfer coefficient on overpotential. In the Butler-Volmer model, the transfer coefficient should remain constant at all overpotentials. For the Marcus model, however, slopes are changing; this means that the transfer coefficient varies with potential. In other words, Marcus the model predicts a potential-dependent transfer coefficient. Finklea noted that a fifth order polynomial can fit the potential dependence of the transfer coefficient predicted by Marcus theory. ${ }^{10}$ Details about the potential dependence of the transfer coefficient will be given in Chapter 3 . 


\subsection{Reorganization Energy.}

As seen in Figure 1.8, Tafel plots begin to show curvature at large overpotentials and, provided that this curvature is significantly large, the reorganization energy can be estimated by fitting Tafel plots to theoretical plots of various reorganization energies (working curves). The definition of the reorganization energy has already been given in Section 1.5. For a simple electron transfer reaction, the total reorganization energy $(\lambda)$ emanates from two sources: ${ }^{7}$

$$
\lambda=\lambda_{\mathrm{is}}+\lambda_{\mathrm{os}}
$$

$\lambda_{\text {is }}$ is the inner sphere reorganization which is the energy associated with changes in the equilibrium internal structure (changes in vibrational modes and equilibrium bond lengths and force constants), as the oxidation state changes. In order to calculate $\lambda_{\text {is, }}$, molecular force constants and changes in bond lengths must be measured. If changes in bond lengths and bond force constants are small, as a result of a change in oxidation state, then $\lambda_{\text {is }}$ is expected to be small. $\lambda_{\text {is }}$ may be strongly dependent on the oxidation state of the redox molecule.

The outer sphere component $\left(\lambda_{\mathrm{os}}\right)$ consists of the changes in the equilibrium solvation sphere around the redox molecule and can be estimated from the dielectric continuum theory: ${ }^{10}$

$$
\lambda_{o s}=(\Delta e)^{2}\left(\frac{1}{D_{o p}}-\frac{1}{D s}\right)\left(\frac{1}{2 a}-\frac{1}{2 r}\right)
$$

where $D_{\text {op }}$ and $D_{s}$ are the optical and static dielectric constants of the electrolyte respectively, $\Delta \mathrm{e}$ is the charge transferred between the electrode and the redox molecule, $a$ is the mean radius of the redox molecule, and $r$ is the thickness of the spacer between the redox center and the electrode. $\lambda_{\mathrm{os}}$ is independent of the oxidation state, but it is clear 
from Equation 1.21 that a large ion in a solvent with low static dielectric constant (low polarity) at a short distance from the electrode will lead to smaller outer-sphere reorganization energy.

Some heavy metals such as osmium and ruthenium generally have small $\lambda_{\text {is }}$ because the bond lengths and bond strength do not change much between the +2 and +3 oxidation states. As a result, the total reorganization energy is dominated by the outer sphere reorganization energies which are equal for both the oxidized and reduced forms. Equal reorganization energies between the oxidized and reduced forms of the redox couple lead to symmetrical Tafel plots and an $\alpha(0)$ value of 0.5 .

However, it is also possible for the reduced form of the redox couple to have a different total reorganization energy from its oxidized counterpart if the inner sphere reorganization energy is a large fraction of the total reorganization energy. This leads to asymmetric Tafel plots and $\alpha(0)$ values which deviate from 0.5 . The plots of $\alpha$ vs $\eta$ are linear with different slopes for the anodic and cathodic branch. A simulation by Finklea confirmed this behavior. Details of the simulation are given below:

\subsection{Data interpretation and treatment using Marcus DOS model when the reorganization energies for the reduced and oxidized species are different.}

The analysis was based on a presentation given by Hupp and Weaver ${ }^{13}$ for a redox couple anchored to an electrode through a SAM. Initially, electron transfer is assumed to occur only at the Fermi level.

Consider a generic simple electron transfer half reaction (Ox $\left.+\mathrm{e}^{-} \leftrightarrow \mathrm{Red}\right)$. The free energies for the Ox and Red forms are assumed be proportional to the square of the 
reaction coordinate (parabolic dependence), $X$. The plot of free energy $(G)$ vs reaction coordinate X shown below in Figure 1.9:

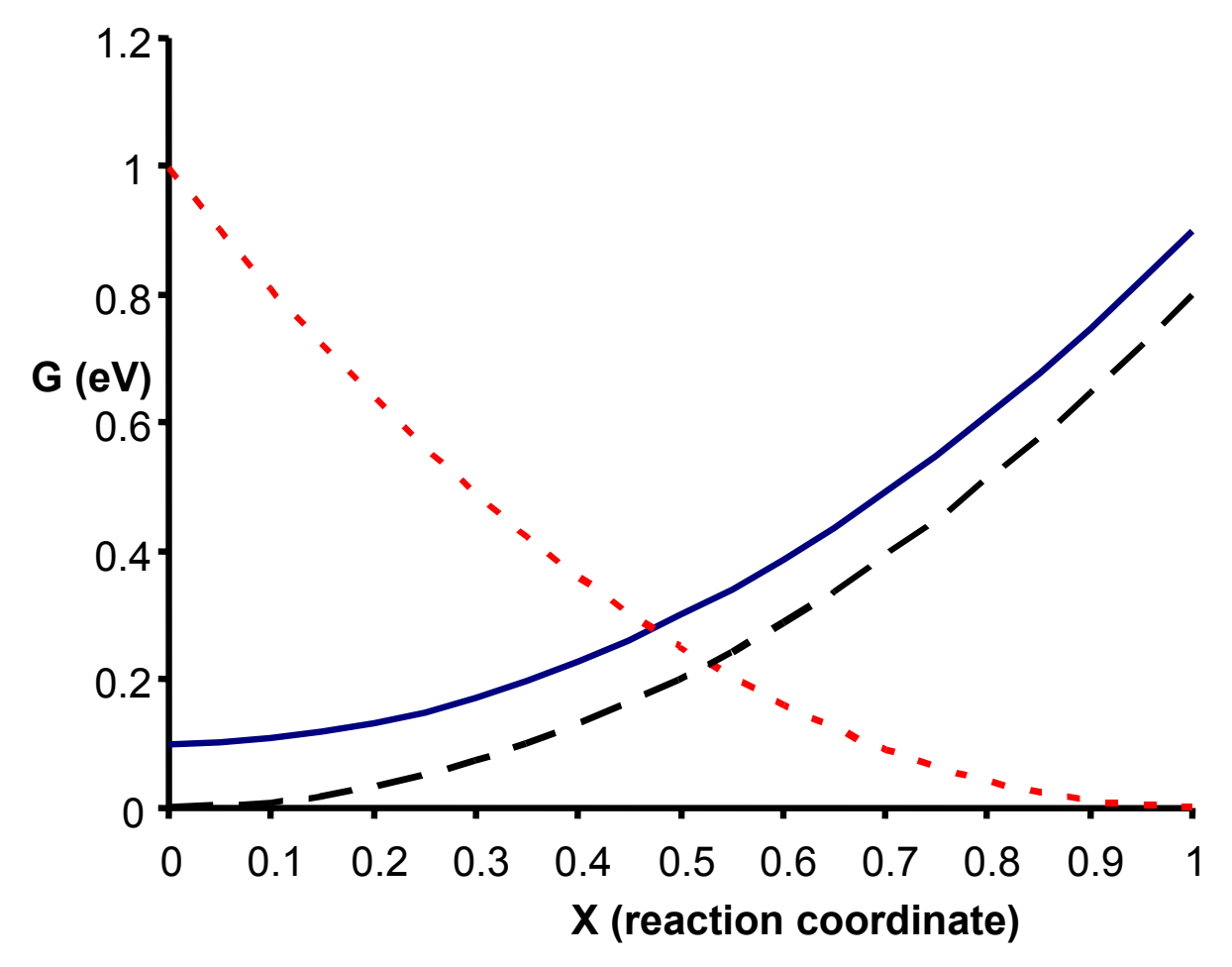

Figure 1.9. Generic variation of free energy with reaction coordinate for a simple electron transfer half reaction. The long dotted line is the free energy of the Ox form plus the free energy of the electron at $\eta=0$. The short dashed line is the free energy of the Red form. The solid line is the free energy of the $\mathrm{Ox}$ form and electron at $\eta=0.1 \mathrm{~V} . \lambda_{\mathrm{Ox}}=0.8 \mathrm{eV}$, $\lambda_{\text {Red }}=1.0 \mathrm{eV}$.

If the left parabola is defined for $\mathrm{Ox}+\mathrm{e}^{-}$,and the right parabola is defined for Red, the free energies of the two components can be represented by the following expressions:

$$
\begin{gathered}
\mathrm{G}_{\mathrm{Ox}}=\lambda_{\mathrm{Ox}} \mathrm{X}^{2}-\mathrm{F} \eta \\
\mathrm{G}_{\text {Red }}=\lambda_{\text {Red }}(1-\mathrm{X})^{2}
\end{gathered}
$$


The curve for $\mathrm{G}_{\mathrm{Ox}}$ (black dash or solid line) is defined by the Ox reorganization energy. The free energy of the electron is defined via the electrode overpotential. $\left(\eta=E-E^{0}\right)$. At the formal potential, the minima of the two curves are equal and $\Delta \mathrm{G}=0$. Applying a potential shifts the $\mathrm{G}_{\mathrm{Ox}}$ curve up or down depending on the sign of the applied overpotential. If a more negative overpotential is applied the $\mathrm{G}_{O x}$ curve shifts upwards and a more positive overpotential causes the curve to move downwards.

In order to calculate the cathodic and anodic rate constants, the height of the point of intersection of the cathodic and anodic free energy curves must be calculated. Let $\mathrm{X}=$ $\mathrm{X}^{\prime}$ at the intersection.

Then:

$$
\begin{aligned}
& \mathrm{G}_{\mathrm{Ox}}\left(\mathrm{X}^{\prime}\right)=\mathrm{G}_{\mathrm{Red}}\left(\mathrm{X}^{\prime}\right) \\
& \lambda_{\mathrm{Ox}} \mathrm{X}^{\prime 2}-\mathrm{F} \eta=\lambda_{\mathrm{Red}}\left(1-\mathrm{X}^{, 2}\right) \\
& \mathrm{X}^{, 2}\left(\lambda_{\mathrm{Red}}-\lambda_{\mathrm{Ox}}\right)-2 \lambda_{\mathrm{Red}} \mathrm{X}^{\prime}+\left(\lambda_{\mathrm{Red}}+\mathrm{F} \eta\right)=0
\end{aligned}
$$

Solve by quadratic solution: $\mathrm{A}=\lambda_{\text {Red }}-\lambda_{\mathrm{Ox}}, \mathrm{B}=-2 \lambda_{\text {Red }}, \mathrm{C}=\lambda_{\text {Red }}+\mathrm{F} \eta$

$$
X^{\prime}=\frac{-B-\sqrt{B^{2}-4 A C}}{2 A}
$$

And $\Delta \mathrm{G}_{\mathrm{Ox}} *=\lambda_{\mathrm{OX}} \mathrm{X}^{2}$

Note that only the negative solution of Equation 1.28 is used because it defines the energy minimum.

Special case: When both reorganization energies are equal, then

$$
\begin{gathered}
X^{\prime}=\frac{(\lambda+F \eta)}{2 \lambda} \\
\Delta G_{O x}^{*}=\frac{(\lambda+F \eta)^{2}}{4 \lambda}
\end{gathered}
$$


$k_{c}=P e^{-\frac{(\lambda+F \eta)^{2}}{4 \lambda R T}}$

Likewise. $k_{a}=P e^{-\frac{(\lambda-F \eta)^{2}}{4 \lambda R T}}$

where $\mathrm{P}$ is the pre-exponential term.

As mentioned above, the preceding Equation corresponds to electron transfer at the Fermi level only. However, the Marcus DoS model integrates the density of states of the Ox molecules $\left(\mathrm{D}_{\mathrm{OX}}\right)$ with the density of occupied states in the metal electrode over all energies. Comparison of Equation 1.32 with Equation 1.18a leads to the following identities:

$\mathrm{RT}=\mathrm{k}_{\mathrm{B}} \mathrm{T}(0.02569 \mathrm{~V}$ at room temperature $) \quad \mathrm{F} \eta=\mathrm{e} \eta$

Also, the energy term in the Gaussian is equivalent to electron transfer at all energies. In order to include all possible energies, it is necessary to incorporate the energy term in the overpotential term:

$$
\mathrm{G}_{\mathrm{c}}=\lambda_{\mathrm{a}} \mathrm{X}^{2}-\mathrm{e} \eta+\varepsilon
$$

Hence, $\quad \mathrm{C}=\lambda_{\mathrm{c}}+\mathrm{e} \eta-\varepsilon$

Tafel plots $(\ln (\mathrm{k})$ vs $\eta)$ obtained from this analysis are symmetrically curved in the anodic and cathodic branches. The transfer coefficient $\alpha$ (from the standard ButlerVolmer formalism) is dependent on overpotential. A plot of $\alpha$ vs $\eta$ is linear with a slope of $F /(4 \lambda)$ and an intercept $\alpha(0)$ of 0.5 .

However, when the Ox and Red reorganization energies are different, then Equations $1.28,1.29$ and 1.35 must be used to calculate $\mathrm{D}_{\mathrm{Ox}}$. Once again assuming that 
electron transfer only occurs at the Fermi energy, a simulation is performed to calculate Tafel data. Figure 1.10 is an example of such a simulation.

Figure 1.10: Tafel Plot for different reorganization energies. $\lambda_{\mathrm{Ox}}=0.7 \mathrm{eV}, \lambda_{\mathrm{Red}}$ $=1.0 \mathrm{eV}$. The symbols are calculated rate constants at specific overpotentials. The line simply connects the symbols.

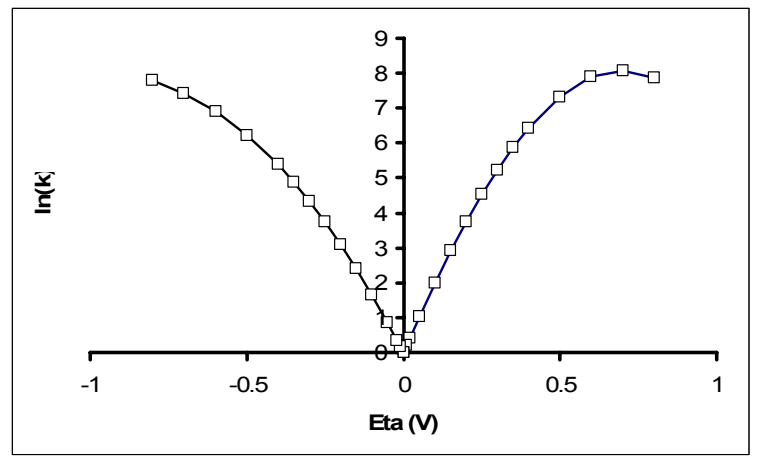

The plot was obtained by using the following $\lambda$ values: $\lambda_{\mathrm{Ox}}=0.7 \mathrm{eV}, \lambda_{\mathrm{Red}}=1.0$ $\mathrm{eV}$. These values were deliberately selected with $\lambda$ value for the reduced species being larger than that for the oxidized species to match experimental observations of this study. At equal absolute overpotentials, the anodic branch of the Tafel plot is steeper than the cathodic branch. In principle, this Tafel plot could be fit to experimental Tafel plots to extract the reorganization energies.

However, a more convenient way to obtain reorganization energy is from a plot of $\alpha$ vs $\eta$ plot (Figure 1.11). The two branches appear to be nearly linear with different slopes and an intercept $\alpha(0)$ less than 0.5 . The slope of the positive branch (fitted between +0.15 and $+0.60 \mathrm{~V}$ ) is $0.352 \mathrm{~V}^{-1}$. The reorganization for each redox species is easily calculated using the formula slope $=F / 4 \lambda$. The slope corresponds to a $\lambda$ value of $0.71 \mathrm{eV}$, which is closer to the $\lambda$ for the oxidized species. In a similar manner, the corresponding slope of the negative branch $\left(0.258 \mathrm{~V}^{-1}\right)$ yields a reorganization energy of $0.97 \mathrm{eV}$, which is close to $\lambda$ for the reduced species. The general observation from this simulation is that, 
for the case of a small difference between large reorganization energies, the slope at positive $\eta$ gives a good approximation of the reorganization energy of the Ox species, while that at negative $\eta$ closely approximates the reorganization energy of the Red species. The intercepts of the linear regression fits ( 0.446 for positive $\eta, 0.449$ for negative $\eta)$ agree very well with the actual value of alpha(0) $(0.456)$.

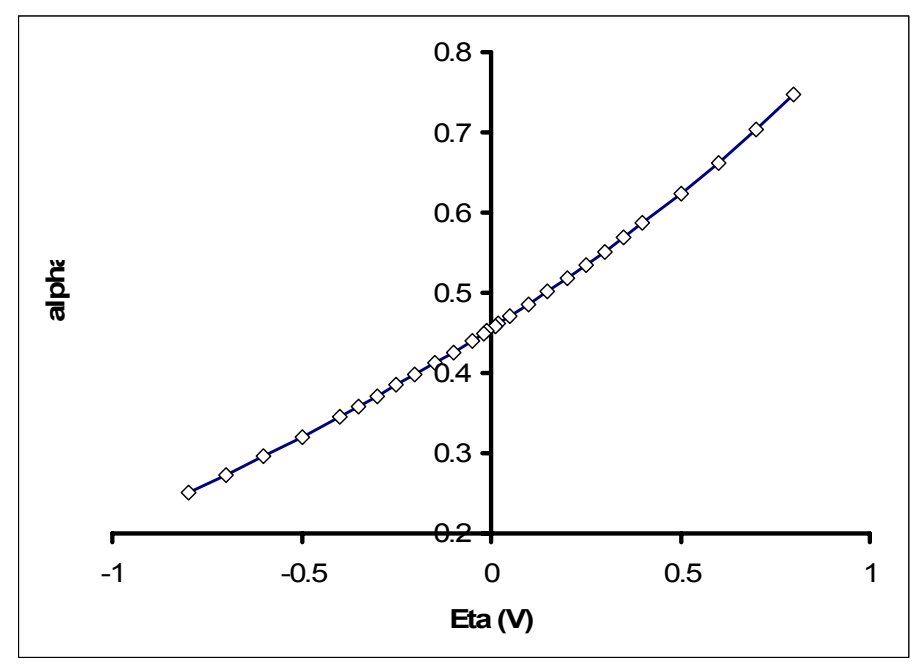

Figure 1.11. $\alpha$ vs $\eta$ for a case of different reorganization energies. $\lambda_{\mathrm{Ox}}=0.7 \mathrm{eV}, \lambda_{\mathrm{Red}}=1.0$ $\mathrm{eV}$. Notice the different slope for each branch.

Electron transfer takes place over a range of energies near the Fermi energy. For the case of equal reorganization energies, the cathodic rate constant is typically evaluated by Equation 1.17. ${ }^{14}$

For the case of different reorganization energy, the cathodic rate constant is obtained by adding the energy $\varepsilon$ to the values calculated for $\Delta \mathrm{G}_{\mathrm{Ox}} *$ obtained in Equation 1.29 and multiplying that value by the value of the Fermi function followed by 
integrating over all energies. The slopes of the $\alpha$ vs $\eta$ plots can be converted to the reorganization energies of the Ox and Red species. Table 1.1 shows the values obtained at explicit reorganization energies.

\begin{tabular}{|l|l|l|l|l|l|l|l|l|}
\hline$\lambda(\mathrm{eV})$ & 0.5 & 0.6 & 0.7 & 0.8 & 0.9 & 1.0 & 1.2 & 1.4 \\
\hline slope $\left(\mathrm{V}^{-1}\right)$ & 0.414 & 0.352 & 0.308 & 0.273 & 0.246 & 0.224 & 0.189 & 0.164 \\
\hline
\end{tabular}

Table 1.1: Slopes of the $\alpha$ vs $\eta$ plot as a function of $\lambda$.

For reorganization energies between 0.5 and $1.4 \mathrm{eV}$, the slope can be calculated by the following empirical Equation:

Slope $=0.242(\lambda)^{0.836}-0.0188$

The reorganization energy can be obtained from slopes of $\alpha$ vs $\eta$ plots by inversion of this Equation.

\subsection{Test for Marcus DoS Theory}

Experiments to test the predictions made by Marcus theory benefited greatly from the use of Self-assembled Monolayers. As already discussed, this was mainly due to the benefits of Self-assembled Monolayers such as absence of mass transfer, absence of double layer effects and the lowering of the standard rate constant to experimentally measurable regions. The standard rate constant is decreased because anchoring the redox molecule on one end of the SAM increases the distance between the redox molecule and the electrode leading to poor electronic coupling between metal states and redox center states. As a result, rate constants can be measured up to large overpotentials, in excess of $0.5 \mathrm{~V}$. 
Experiments have been conducted to test the Marcus DoS theory using SAMs for many simple electron transfer reactions ${ }^{3,5,8,9,10,11,12}$. One study involved measuring the standard rate constant as a function of the chain length of the SAM using ferrocene as the redox probe. ${ }^{9,15,16}$ A plot of $\ln \left(\mathrm{k}_{\mathrm{s}}\right)$ vs chain length is linear with a slope of -1.1 per $\mathrm{CH}_{2}$ group. The value of the slope gives the electron tunneling parameter, $\beta$. According to Marcus, this parameter is controlled by electronic coupling through the SAM chain (through bond tunneling). Replacing one $-\mathrm{CH}_{2}$ on the SAM chain with a heteroatom such as $\mathrm{O}$ or with a double bond leads to poor orbital overlap along the chain, which lowers the standard rate constant. Using a conjugated SAM chain lowers $\beta$ and increases electron tunneling dramatically, leading to high $\mathrm{k}_{\mathrm{s}}$ values.

Another test for the Marcus theory involves Tafel plots. ${ }^{9,10,13}$ For rutheniumpentaaminepyridine complex attached to a $\mathrm{C}_{16}$ chain alkanethiol, Tafel plots were symmetrical and slightly curved. The data were fit to Marcus theory to obtain the reorganization energy values between $0.45-0.7 \mathrm{eV}$ depending on the chain length of the redox spacer. ${ }^{15}$ Longer chain SAMs gave higher $\lambda$ values than shorter chain SAMs. In the same study, a plot of $\ln \left(\mathrm{k}_{\mathrm{s}}\right)$ vs chain length of SAM yielded a tunneling parameter of 1.06 \pm 0.04 in very good agreement with the through bond mechanism. In another study, Ravenscroft and Finklea observed symmetric Tafel plots in different aqueous electrolytes. The Tafel plots were fit to Marcus theory to get $\lambda$ values ranging between $0.7-0.8 \mathrm{eV}{ }^{9}$

Yet another experimental verification of the Marcus theory involved measuring the standard rate constant as a function of metal ${ }^{9}$, using the ruthenium complex as a redox molecule. The redox molecule was attached to a $\mathrm{C}_{16}$ chain and used to measure the 
standard rate constant on platinum, gold and silver. The density of states for platinum at the Fermi level is roughly an order of magnitude higher than for gold and silver. The conduction band for platinum has contribution from both the "s" and "d" orbitals, while that for gold and silver is made up of only "s" orbitals. However, the standard rate constant was nearly the same on platinum, gold and silver. To explain this observation, Marcus ${ }^{17}$ noted that metal states derived from "s" atomic orbitals extended further away from the metal than those derived from "d" atomic orbitals. This is because "d" orbitals are closer to the nucleus and therefore are more tightly held by the nucleus than the "s" orbitals. Consequently, tunneling was largely controlled by electronic coupling of the redox molecule with the "s" orbital electronic states of the metal electrode 


\section{CHAPTER 2: INTRODUCTION TO SELF-ASSEMBLED MONOLAYERS}

(SAMs).

Self-assembled monolayers are monomolecular layers of organic chains formed by spontaneous adsorption of the relevant organic molecules on the surfaces of electrodes. They have found widespread applications as surfaces modifiers and as spacers between redox species and electrodes. Because of the importance of long range electron transfer in many biological systems such as proteins, enzymes and DNA, it is vital to understand the factors that control long range electron transfer, and SAMs provide an excellent means to do that.

SAMs are formed from molecules that contain a head group with a high affinity for the substrate, an alkyl chain spacer which packs into a dense structure, and a tail or terminal group which can be tailored for a specific application. SAMs can be formed from the vapor phase or solution on a variety of surfaces such as silica, gold, silver and palladium. ${ }^{18}$ This discussion will focus on SAMs formed from solution phase because it is convenient and sufficient for most applications. Also, since gold is the most studied material, for historical reasons, the discussion will also focus on assembly on gold surfaces. Gold is inert, which makes it a metal of choice for a variety of applications. It does not react with most chemicals or oxygen, neither does it oxidize at temperatures below its melting point. ${ }^{18,19}$ Although SAMs can be formed from a variety of functional materials such as organosilicon, carboxylic acid, amines, disulfides and alkanethiol, this discussion will be mainly based on SAMs formed from alkanethiols.

Alkanethiols have had widespread applications in modification of metal surfaces. The sulfur head group on these molecules has a high affinity for metal surfaces and when 
alkanethiol molecules are brought in the vicinity of a gold electrode, they spontaneously bind onto the gold surface, forming a strong Au-S bond. The mechanism of adsorption is not well established, but it is believed that formation of SAMs involves a complex balance of the energetics of the Au-S bond and the non-covalent interchain interactions among the organic groups. ${ }^{18}$ The hydrogen on the alkanethiol is believed to be lost as hydrogen or oxidatively converted to water if oxygen is present in the thiol solution. The loss of the hydrogen atom creates an alkyl thiolate $\left(\mathrm{RS}^{-}\right)$group which binds onto the gold surface, possibly through some ionic interactions. Whatever the exact mechanism of this process, the resulting $\mathrm{Au}-\mathrm{S}$ interaction is strong enough to retain the alkanethiol chain on the surface of gold and form a durable bond. The bond energy for the Au-S bond is believed to be around $40-50 \mathrm{kcal} / \mathrm{mol}^{18,19}$

The formation of a SAM occurs in two stages; the first stage involves the chemisorption of the sulfur group on the gold surface. This process is fast and highly exothermic; it takes place within a few seconds to minutes of exposure of substrate to a thiol solution. Because of the high affinity of the sulfur group for gold, it displaces any extrinsic materials from the gold surface very readily. ${ }^{18}$ As a result, all available surface active sites on the gold surface are occupied by the SAM. This initial chemisorption process brings the thiol molecules close together so much that short range inter-chain Van der Waals forces begin to operate, forcing the molecules to orient themselves vertically, with a contact angle that varies depending on the spacing of the head-groups as well as substrate type and crystallographic face exposed. For $\mathrm{Au}(111)$, the contact angle is approximately 30 degrees. ${ }^{20}$ This inter-chain interaction begins a slow second phase of reorganization, which can take up to a few days. Typically, the whole deposition process 
is done over 15 to 24 hours. To maximize the degree of packing and order, the organic molecules arrange themselves in an all-trans configuration resulting in a rigid, highly ordered structure. SAMs are generally more ordered than liquids or amorphous solids. ${ }^{20}$

\subsection{Preparation}

The general preparation protocol for SAMs is to immerse a clean freshly prepared electrode into a dilute ethanolic solution of thiols for a period of 15 to 24 hours. Typical concentrations range from 1 to $10 \mathrm{mM}$, although lower concentrations can be used. In order to get a high quality SAM and to minimize defects as well as to get reproducible functional behaviors, several factors must be controlled. Some of the factors that need to be monitored include solvent type and purity, concentration of thiol and immersion time, purity of thiols, temperature, cleanliness of substrate surface as well as oxygen content of the solution. This section will look at each of these factors more closely.

A variety of solvents have been used to prepare self-assembled monolayers. Some of the solvents used include tetrahydrofuran, dimethylformamide, toluene, acetonitrile, cyclooctane and ethanol. Although the limiting coverage and wettability of the SAMs formed from these solvents are not significantly different, ethanol has been the most widely used solvent. ${ }^{18}$ Compared to the other solvents, ethanol is less toxic, cheap and available in high purity. Ethanol can also solvate a wide range of alkanethiols with varying degrees of polarity and chain length. The effect of solvent on the mechanism of self-assembly is not well understood. ${ }^{18}$

Temperatures above $25^{\circ} \mathrm{C}$ have been found to promote better quality, defect free SAMs. ${ }^{21}$ This is because, at higher temperatures, solvent molecules or other materials occupying active sites on the substrate can be desorbed at a faster rate than at lower 
temperatures. Upon binding on the substrate, SAM molecules undergo lateral rearrangements and chain reorganization to form a well ordered, densely packed structure. This process has been found to be faster at elevated temperatures. ${ }^{18}$ Adsorption and reorganization of SAMs occurs during the first few minutes of formation and it is during this period that temperature is particularly important. ${ }^{22}$

The purity of the adsorbing material also has an effect on the quality of the monolayer formed. Common contaminants in thiols include disulfides and oxidized polar compounds such as sulfonates. However, if the levels of these compounds is less than five percent, the structure or formation of the SAM is not adversely effected. ${ }^{21}$

There is not much known about the effect of oxygen content on rate of formation of a SAM or the structure of the SAM. Degassing the deposition solvent using argon or nitrogen before preparing the thiol and maintaining an inert atmosphere over it during SAM formation has been suggested to improve the reproducibility of SAM preparation. Sulfur moieties are believed to be oxidized by oxygen to sulfonates under room temperature conditions. ${ }^{18}$ Ozone has also been suspected to play a significant role in the oxidation of SAMs under ambient conditions. ${ }^{23}$ However, this problem is more common for SAMs prepared on palladium, silver and copper than it is for gold. ${ }^{24}$

The cleanliness of the substrate plays a critical role in the formation of SAMs. Before the adsorbing molecules of the thiol can bind onto the surface of the substrate, any adventitious materials on the substrate surface must be displaced. This includes solvent molecules and other impurities. The rate of desorption of these extrinsic materials affect the kinetics of formation of SAMs. The most common and effective method for cleaning substrates, especially gold, involve etching the gold with strongly oxidizing "piranha" 
solution (a 3:1 mixture of concentrated sulfuric acid and hydrogen peroxide-30\%) at about $100{ }^{\circ} \mathrm{C}$ (NOTE: This solution is corrosive and reacts violently with organic compounds: it should be kept away from organic compounds). This chemical step is usually followed by an electrochemical step to remove any residual oxide, resulting in a reproducibly clean surface. ${ }^{5,25}$ After this step, the electrode is usually rinsed with lots of water followed by the solvent in which deposition is to be carried out (typically ethanol). After cleaning, deposition must be done within a reasonably short period of time before contamination occurs again. ${ }^{18}$ Drying the electrodes by blowing with an ultra pure inert gas such as argon or nitrogen before immersing in deposition solution has also been found to be useful in removing solvent molecules from the electrode surface. ${ }^{5}$ Both thiols and disulfides form SAMs of similar structure on clean electrode surfaces. However, disulfides are more difficult to use in solution than thiols because of their low solubility.

Another important factor to consider is the concentration and immersion time for thiols in solution. There is an inverse relationship between the immersion time and the concentration of the adsorbing material, with dilute adsorbates requiring longer deposition times than more concentrated ones. To form a dense SAM $\left(6 \times 10^{14}\right.$ molecules $/ \mathrm{cm}^{3}$ ), concentrations above $1 \mu \mathrm{M}$ are usually required. ${ }^{18}$ For extremely dilute solutions, the effect of impurities and other sulfur containing impurities becomes significant. Evidence from spectroscopy suggest that the average properties of SAMs prepared from concentrated solutions $(\sim 1 \mathrm{mM})$ for time periods between 12 to 18 hours do not change over time. However evidence from electrochemistry ${ }^{26}$ and $\mathrm{STM}^{27}$ indicate that the structure of SAMs continues to develop over immersion times up to ten days. 
This suggests that the surface coverage continue to increase and pinholes and defects in the chain are minimized by longer immersion times.

One of the challenges faced in SAM deposition is the formation of more than a single layer of thiol molecules on the surface. This occurs for thiol molecules with certain functional groups such as carboxylic acid or amine as terminal groups. These groups are able to undergo hydrogen bonding, leading to multilayer formation. Kim and coworkers observed that immersion times greater than four hours in millimolar solutions could lead to mutlilayers. ${ }^{28}$ This time could be longer in more dilute solutions. Jiang and others ${ }^{29}$ observed that rinsing the thiol in a weak acid followed by a weak base removed the second layer and resulted in a good quality monolayer.

\subsection{Electron transfer on SAM modified electrodes.}

Because of the ease with which SAMs are formed, they have found widespread applications as surface modifiers. The discussion here will be limited to the use of SAMs to modify electrodes in the study of long range electron transfer kinetics. Their popularity in this area stems from the fact that SAMs are stable over a wide range of electrode potentials and electrolyte compositions. ${ }^{30}$

Two approaches are normally used to study the rate of electron transfer using SAMs. The first approach involves attaching the redox molecule to the electrode via the self-assembled monolayer chain as shown in Figure 2.1(a) The resulting SAM is called an electroactive SAM. Attachment of the redox molecule to the SAM can be done either covalently or ionically using the appropriate chemical methods.

By using electroactive monolayers, complications due to mass transfer are eliminated because the redox molecule is at a fixed distance from the electrode and its 
concentration remains constant. There are no redox molecules in solution. Because mass transfer is absent, the Equations describing the current-voltage relationship do not have the diffusion component of current and this simplifies the kinetics. ${ }^{7}$ Also double layer effects are absent because alkanethiols form a hydrocarbon layer of low dielectric constant around the electrode. ${ }^{30}$ The dielectric constant of this layer approaches that of a pure hydrocarbon. The SAM replaces the Helmholtz layer, thereby forming a bridge between the electrode and the electrolyte. This structure reduces the capacitance of the electrode/SAM/electrolyte interface by more than an order of magnitude compared to that of a bare electrode/electrolyte interface. The capacitance becomes nearly independent of applied potential or composition of electrolyte. ${ }^{30}$

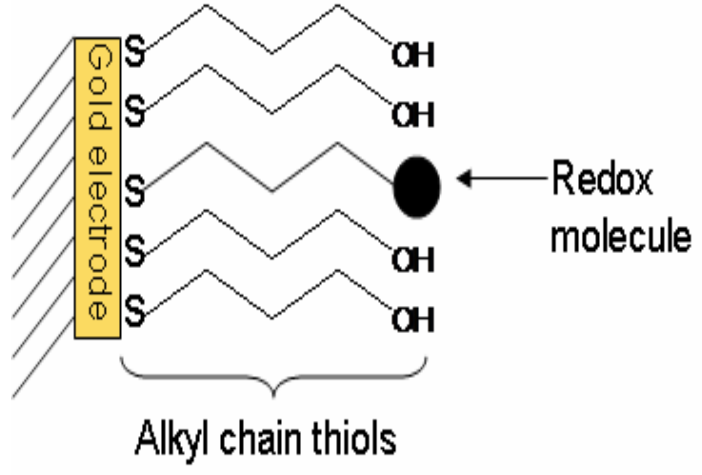

Figure 2.1.(a). Electroactive Monolayer

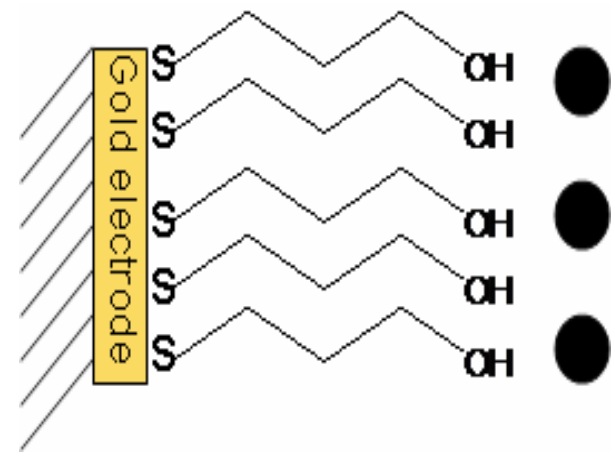

(b). Blocking Monolayer

It is also interesting to note that the rate constants for electron transfer can be easily controlled by controlling the length of the thiol. By increasing the chain length of the monolayer, the rate constant can be decreased by many orders of magnitude compared to that of a bare electrode. ${ }^{30}$ The significance of this fact is that the rate of electron transfer can be reduced to ranges that can be conveniently measured using less 
sophisticated equipment. Rate constants can be measured to very large overpotentials using monolayer coated electrodes.

An alternative approach involves having the redox molecule freely diffusing in solution; this kind of SAM configuration is called a blocking SAM, Figure 2.1(b). Blocking SAMs have the same advantages as electroactive SAMs. A densely packed monolayer will block electrolyte ions as well as redox molecules from reaching the electrode. Electrode oxidation can be suppressed up to $0.5 \mathrm{~V}$ beyond the regular potential limits. ${ }^{30}$ For a perfectly blocking SAM, electron transfer occurs by tunneling and any redox species whose formal potential is accessible can be studied. The electron must tunnel across the whole length of the SAM chain at a rate which is exponentially dependent on overpotential. To achieve these attributes with blocking SAMs, the monolayers must be perfectly blocking. However, the concept of perfect blocking is an idealized situation; for many practical purposes, blocking SAMs show defects and pinholes which is often a challenge in their use to study electron transfer. A defect occurs due to a collapse or loose packing in the SAM structure, resulting in a redox molecule being able to get closer to the electrode as shown in Figure 2.2 (a). A pinhole occurs when a portion of the monolayer coated electrode surface is completely exposed to electrolyte ions and redox species, Figure 2.2 (b). Redox molecules are able to penetrate the monolayer at these sites. Electron shuttling across these sites is fast and masks currents originating from tunneling across the monolayer thickness (the preferred pathway). ${ }^{15}$ 


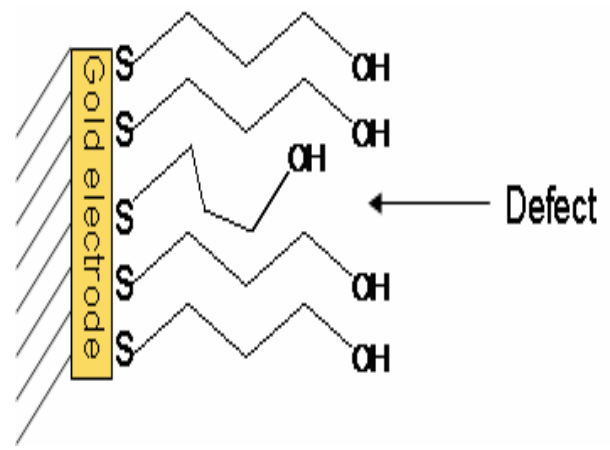

(a). Defects in SAMs.

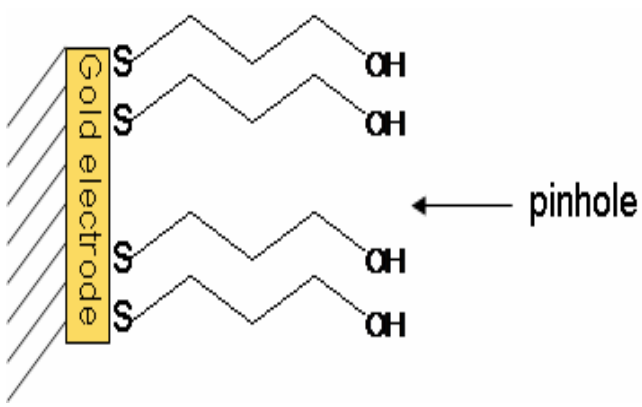

(b). pinhole

Figure 2.2. Blocking SAMs showing (a). defects and (b). pinholes in self-assembled monolayers.

Cyclic voltammetry is a convenient diagnostic tool for defects and pinholes. Because redox species or other ions are able to reach the electrode surface or get close to it at pinhole and defect sites, the current flux at those sites increases greatly. ${ }^{31}$ This property is manifested as current peaks and plateaus at potentials close to the formal potentials of the redox couple. These peaks and plateaus are a sign that electron transfer to the electrode surface is mass-transfer-limited..$^{30,31}$

Defects and pinholes can be minimized by alternately immersing the electrode into the deposition solution and rinsing with pure solvent. ${ }^{31}$ This process removes any loosely bound thiols and replace them by strongly binding molecules. Electrochemical cycling in the appropriate potential region followed by re-immersion in deposition solution has also been found to minimize defects and pinholes.

Because of problems with defects and pinholes in blocking SAMs, electroactive SAMs have been more widely used than blocking SAMs. Electroactive SAMs are less sensitive to the effects of pinholes and defects. ${ }^{26,32}$ This is because the redox center is 
held at some fixed distance away from the electrode, and will likely only exchange an electron with the electrode across the full length of the SAM. There are no redox species floating in solution. The discussion from hereon will focus on electroactive SAMs (redox center covalently attached to SAM).

\subsection{Review of electron transfer kinetic measurements using mixed SAMs.}

The formation of mixed SAMs is a strategy often used to control coverage of the redox species in the study of electron transfer kinetics. This is often achieved by coadsorption of a mixture electroactive and diluent thiols. A diluent thiol does not have a redox molecule attached to it and its purpose is to interpose between adjacent redox species. This keeps the redox species as far apart as possible to minimize any electrostatic interactions between them. More details about mixed SAMs are given in chapter 5 .

As already mentioned in Chapter 1, the electronic coupling parameter $|V|^{2}$ is dependent on the distance between the electron donor and electron acceptor. The effect of SAMs on this parameter follows the tunneling law: ${ }^{31}$

$|V|^{2} \approx e^{-\beta_{r} r}$

where $r$ is the thickness of the SAM or the distance between the redox molecule and the electrode surface, and $\beta_{\mathrm{r}}$ is the distance tunneling parameter. Because of its organic nature (long chain hydrocarbon), the SAM is essentially an insulator. Using the quantum tunneling model, the SAM is treated as a dielectric barrier whose barrier height is determined by the lowest unoccupied molecular orbital (LUMO) of the SAM chain. ${ }^{31,33}$ Under such conditions, electron transfer from the redox center to the electrode would have to follow a through-space electron tunneling mechanism and typical $\beta_{\mathrm{r}}$ values predicted by this model range from 1.3 to $1.8 \AA^{-1} \cdot{ }^{15}$ Experimental evidence, however, 
suggest that electron tunneling occurs specifically along the bridge connecting the electrode to the redox center, which suggest a through-bond electron tunneling mechanism. ${ }^{30,33}$ For SAM chains containing a repeating unit such as a methylene group $\left(-\mathrm{CH}_{2}\right)$, the dependence of the electronic tunneling parameter on the number of repeating units, $m$, can be estimated from calculations. Equation 2.1a is re-written as: $|V|^{2} \approx e^{-\beta_{m} r}$

For through bond tunneling, theory predicts a $\beta_{m}$ value of 1.0 per methylene group, for an alkane chain with at least ten methylene groups. ${ }^{30,33}$ Experimental verification has revealed $\beta_{\mathrm{m}}$ values ranging from 1.0 to 1.2 per methylene group irrespective of the type of redox molecule attached to the electrode or the electrode potential. ${ }^{15,30,34}$

A number of studies has been done for alkanethiol SAMs on gold to measure the standard rate constant, $\mathrm{k}_{\mathrm{s}}$, as a function of chain length in aqueous solutions. ${ }^{8,15,26,35,36}$ The standard rate constant is proportional to the electronic coupling between the redox molecule and the electrode. A semilog plot of $\ln \left(\mathrm{k}_{\mathrm{s}}\right)$ vs. chain length $(m)$ gives $\beta_{\mathrm{m}}$ as the slope. Both blocking SAMs with freely diffusing redox molecules and electroactive SAMs with fixed redox molecules gave $\beta_{\mathrm{m}}$ values in the same range. ${ }^{37}$ These observations suggest that electron transfer across SAM on electrodes can be adequately described by the through-bond mechanism as opposed to the through-space mechanism. The measured values are based on the assumption that the reorganization energy remains constant as the length of the SAM changes. Available data agree with this assumption. ${ }^{30}$

$\beta_{\mathrm{r}}$ values for conjugated systems are lower than those obtained with methylene groups. A study with phenyl-ethynyl group (HS-(p-phenyl-ethynyl-) $m$ with attached ferrocene yielded a value of $0.57 \AA^{-1}$ (vs $B_{r}=1.0 \AA^{-1}$ for alkane thiols). The extended $\pi$ - 
conjugation was believed to electronically couple the redox molecule and electrode more effectively than an alkane chain. ${ }^{30}$

Besides distance, other factors that affect electron tunneling include thr presence of multiple tunneling pathways and heteroatoms or multiple bonds in the alkane chain. ${ }^{35}$, 38

If an oxygen atom is introduced in the alkane chain of a SAM, the total electronic coupling is decreased. Jun Cheng and coworkers ${ }^{38}$ investigated the effect of chain modification on the electronic coupling between the redox molecule and an electrode. The study was done using osmium(III) tris(bipyridine) and ferricyanide as redox probes on a $\mathrm{HS}\left(\mathrm{CH}_{2}\right) n-\mathrm{X}-\left(\mathrm{CH}_{2}\right)_{m} \mathrm{SH}$ SAM, where $\mathrm{X}$ is either oxygen (heteroatom) or a double / triple bond. In each of these cases, there was a significant decrease in electronic coupling.

Finklea and workers ${ }^{35}$ did a systematic study of the influence of multiple paths using a ruthenium pentaamine complex on the following mixed SAMs; (i) matched SAMs where the electroactive and diluent chain lengths are equal (ii) exposed SAMs, where the diluent chain was shorter than the electroactive chain and (iii) buried SAMs where the diluent chain was longer than the electroactive chain. ${ }^{35}$ In each case, the diluent SAM was a carboxy-terminated thiol $\left(\mathrm{HS}\left(\mathrm{CH}_{2}\right)_{m} \mathrm{COOH}\right)$ with number of methylene groups, $m$, ranging from 10 to 15 . Each of the terms matched, exposed and buried describes the position of the redox molecule in relation to the diluent thiol. For example in the exposed case, the redox molecule was freely hanging in the electrolyte, while in the buried case, it was partially or completely buried inside the diluent thiol. For the matched case, the observed tunneling parameter $\left(0.97 \pm 0.03\right.$ pre $\left.\mathrm{CH}_{2}\right)$ agreed very well with other reports with pure alkane chain spacers. The value for the exposed case $(0.83 \pm 0.03)$ was 
higher than expected especially considering gauche conformations introduced by the free motion of the chain extending beyond the densely packed diluent thiol. Gauche conformations are thought to decrease electronic coupling between the redox center and the electrode; therefore, electron tunneling (hence rate constant) was expected to decrease as the diluent chain decreased. ${ }^{35}$ To explain their observations, Finklea and coworkers noted that the free motion of the electroactive chain can bring the redox molecule to the terminus of the diluent thiol for a period long enough for electron transfer to occur. In this case, tunneling would occur via both the electroactive chain as well as the diluent chain, with tunneling via diluent thiol dominating. Due to the random motion of the chain containing the redox molecule, the statistical fraction of the redox species in contact with the diluent thiol at any given instant is low; hence the $\beta_{m}$ value is slightly lower than that for the matched case. For the buried case, a small $\beta_{m}$ value was obtained because there was no significant change in the electronic coupling along the diluent chain, as the diluent chain length changed. 


\section{CHAPTER 3: INTRODUCTION TO PROTON COUPLED ELECTRON TRANSFER (PCET) REACTIONS.}

\subsection{Background}

Reactions in which both an electron and a proton are transferred are often called proton-coupled electron transfer reactions. These types of reactions are common in many biological and chemical systems and have been subject of great interest for the past

decade. ${ }^{39-49}$ Some examples of such processes include photosynthesis, enzymes, flavins and protein function. During photosynthesis, for example, absorption of a photon of light by the leaf chloroplast triggers a cascade of proton motion coupled to electron transfer reactions. Redox-driven proton pumps are the primary events in biological energy transduction in cell respiration. This coupled electron-proton motion creates an electrochemical proton gradient which drives energy requiring reactions like ATP synthesis in the presence of cytochrome c. oxidase, a terminal enzyme in mitochondrial respiration. ${ }^{49}$ A variety of other species such as ribonucleotide reductase enzyme, iron sulfur proteins ${ }^{42}$ and neurotransmitters also involve PCET reactions. In order to understand these processes to a deeper level, a fundamental understanding of the mechanism of proton-coupled electron transfer is needed.

Recent theoretical and experimental models have focused on homogenous systems. $^{39,41,45,49-56}$ Of particular interest are studies by Hammes-Schiffer and Cukier. Although their theoretical analyses differ fundamentally, ${ }^{51}$ both models are based on a redox couple in solution, bridged by a proton transfer interface, ${ }^{41,42}$ such as a protonated water dimer. ${ }^{42}$ Electron transfer is photo-initiated and both the electron and the proton are treated as quantum mechanical particles. Two theoretical models have been proposed to 
explain the observed trends in proton and electron transfer. The stepwise model has been traditionally accepted by electrochemists.

In the stepwise model, the proton transfer and electron transfer steps are treated as distinct and separate events. There could be either a proton transfer step followed by an electron transfer step or vice versa. However, recent studies have shown that the concerted mechanism is also possible. The concerted mechanism combines these two processes into a single event which does not pass through an intermediate ${ }^{19}$. This means that the proton transfer step is electronically coupled to the electron transfer step and both the electron and the proton tunnel from the same donor to the same acceptor molecule. Homogeneous systems are usually complex and require sophisticated equipment and complex models to study them.

The approach adopted in this study involves understanding the mechanism of PCET reactions in heterogeneous systems as a platform for understanding the more complex homogeneous systems. The starting point was a theoretical formulation developed by E. Laviron in a series of papers in the early 1980 s. ${ }^{39,}{ }^{57}$ In her analysis, redox species are assumed to be strongly adsorbed on the surface of an electrode. Laviron's analysis was based on the stepwise model and two key assumptions were made. First, the proton transfer step was assumed to be at chemical equilibrium and separate from electron transfer under all conditions ${ }^{39,45,57}$. The second assumption was that the transfer coefficient is constant and independent of potential. The consequences of each of these assumptions will be explored in later sections. Laviron's formulation is successful in predicting thermodynamic properties of PCET systems. However, as shall be seen later, Laviron's kinetic model has had some limitations in adequately explaining 
experimental observations. Of particular concern is the assumption that the transfer coefficient remain constant at 0.5 at all overpotentials.

Finklea extended Laviron's worked by re-deriving some of Laviron's earlier kinetic predictions. ${ }^{10,58,59}$ Finklea's derivation maintained the first assumption, but allowed the transfer coefficient to vary with potential as predicted by Marcus density-ofstates theory. A simple case where a single electron and a single proton are transferred as shown in Equation 3.1 will be considered.

$O+e^{-}+H^{+} \leftrightarrow R$

$\mathrm{O}$ is the oxidized species and $\mathrm{R}$ is the reduced species.

Laviron's discussion was based on the scheme of squares initially proposed by Albery ${ }^{60}$ (Figure 3.1). The same scheme will be adopted in this analysis. It can be noted that the redox species $\mathbf{O}$ can be converted to $\mathbf{R}$ via three routes, electron transfer followed by proton transfer (OPR), proton transfer followed by electron transfer (OQR) and simultaneous electron-proton transfer (OR). Route $\mathbf{O R}$ is the concerted mechanism while OPR and OQR define the stepwise mechanism which will be the subject of this derivation. Experimental verification of this model will be achieved by using a model 1electron, 1-proton $(1 \mathrm{e}, 1 \mathrm{H})$ osmium aquo complex, as a redox couple.

The scheme in Figure 3.1 describes four equilibria; the vertical lines represent proton transfer steps or acid-base equilibria, which define two thermodynamic parameters $\mathrm{K}_{\mathrm{a} 1}$ and $\mathrm{K}_{\mathrm{a} 2}$. Horizontal lines represent redox equilibria and two thermodynamic parameters $E_{1}^{0^{\prime}}$ and $E_{2}^{0^{\prime}}$ are defined as well as kinetic parameters $\alpha_{1}, \alpha_{2}, \mathrm{k}_{\mathrm{s} 1}, \mathrm{k}_{\mathrm{s} 2}, \lambda_{1}$, and $\lambda_{2}$. The diagonal represent the two equilibria combined -concerted mechanism. The four 
forms of redox species are depicted by $\mathbf{O}\left(\mathrm{Os}{ }^{\mathrm{III}}-\mathrm{OH}\right), \mathbf{P}\left(\mathrm{Os}^{\mathrm{II}}-\mathrm{OH}\right), \mathbf{Q}\left(\mathrm{Os}^{\mathrm{III}}-\mathrm{OH}_{2}\right)$ and $\mathbf{R}$ $\left(\mathrm{Os}^{\mathrm{II}}-\mathrm{OH}_{2}\right) .{ }^{58,59}$

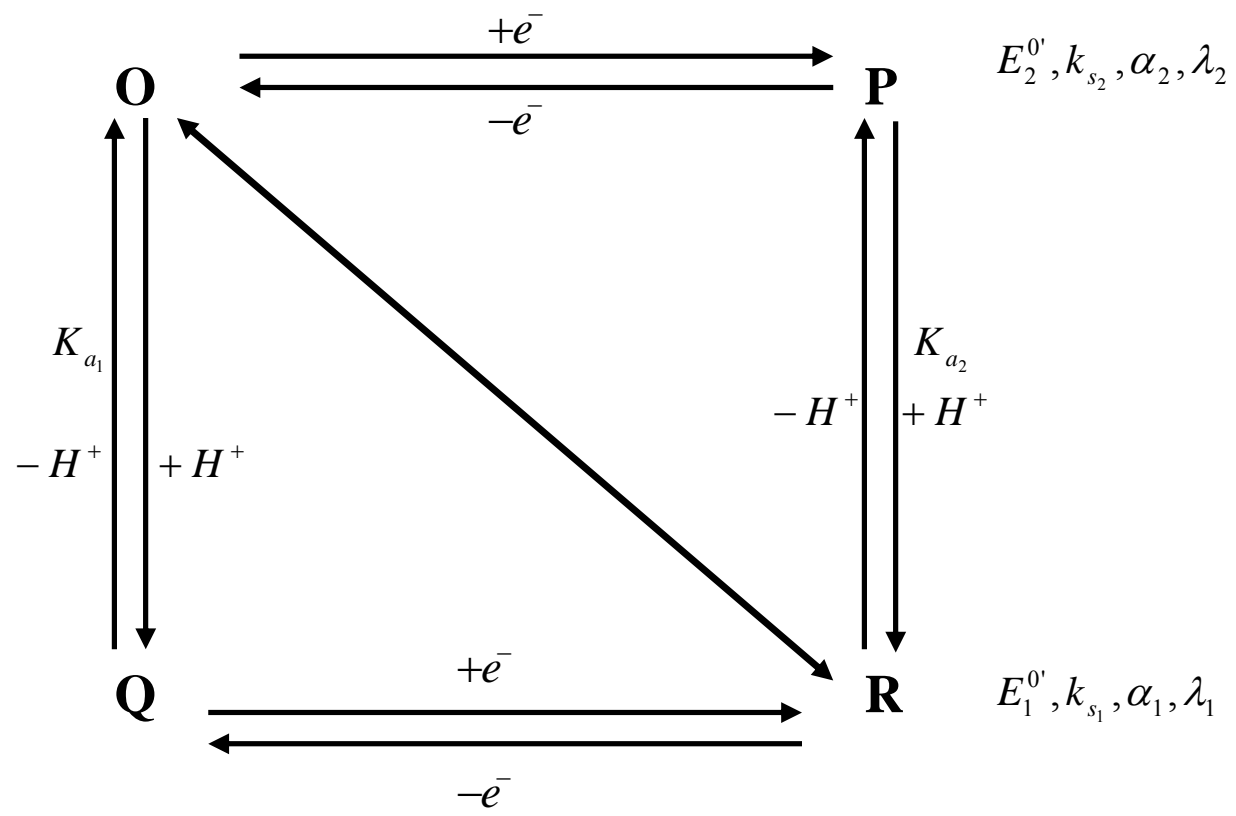

Figure 3.1. 1e,1H square scheme I for PCET reactions.

Since the left hand side of Figure 3.1 is made up of species in higher oxidation states compared to the right hand side, it can be shown using electrostatic arguments that the acid dissociation constant, $\mathrm{K}_{\mathrm{a}}$, decreases going from left to right (the oxidized species is a stronger acid than the reduced species). Likewise, the formal potential becomes more positive from top to bottom. Assuming proton transfer step is at equilibrium, the system must behave like a one-electron reaction with a $\mathrm{pH}$-dependent reversible formal potential $\mathrm{E}^{\mathrm{OR}}$, defined at all $\mathrm{pHs}$ as:

$E^{O R}=E_{2}^{0^{\prime}}+\frac{2.3 R T}{F} \log \left(\frac{1+\frac{\left[H^{+}\right]}{K_{a_{2}}}}{1+\frac{\left[H^{+}\right]}{K_{a_{1}}}}\right)$ 
The two $\mathrm{pK}_{\mathrm{as}}$ and formal potentials are chosen as the thermodynamic parameters such that the separation between the two formal potentials is given by:

$E_{1}^{0^{\prime}}-E_{2}^{0^{\prime}}=\frac{2.3 R T}{F}\left(p K_{a_{2}}-p K_{a_{1}}\right)$

In Laviron's derivation, the potential midway between the two $\mathrm{pK}_{\mathrm{a}} \mathrm{s}, E_{m}=\frac{\left(E_{1}^{0^{\prime}}+E_{2}^{0^{\prime}}\right)}{2}$, was used as the third thermodynamic property leading to a slightly different Equation. ${ }^{57}$ The PCET reaction occurs in the region $\mathrm{pK}_{\mathrm{a} 1}<\mathrm{pH}<\mathrm{pK}_{\mathrm{a} 2}$ and a plot of $\mathrm{E}^{\mathrm{OR}}$ vs. $\mathrm{pH}$ exhibits a Nernstian slope of $-60 \mathrm{mV} / \mathrm{pH}$ in this range (Figure 3.2). This plot also yields the four thermodynamic properties $\mathrm{K}_{\mathrm{a} 1}, \mathrm{~K}_{\mathrm{a} 2}, \mathrm{E}^{0}{ }_{1}$ and $\mathrm{E}_{2}^{0}$ if the flat regions (the regions where the formal potential becomes independent of $\mathrm{pH}$ ) are well defined.

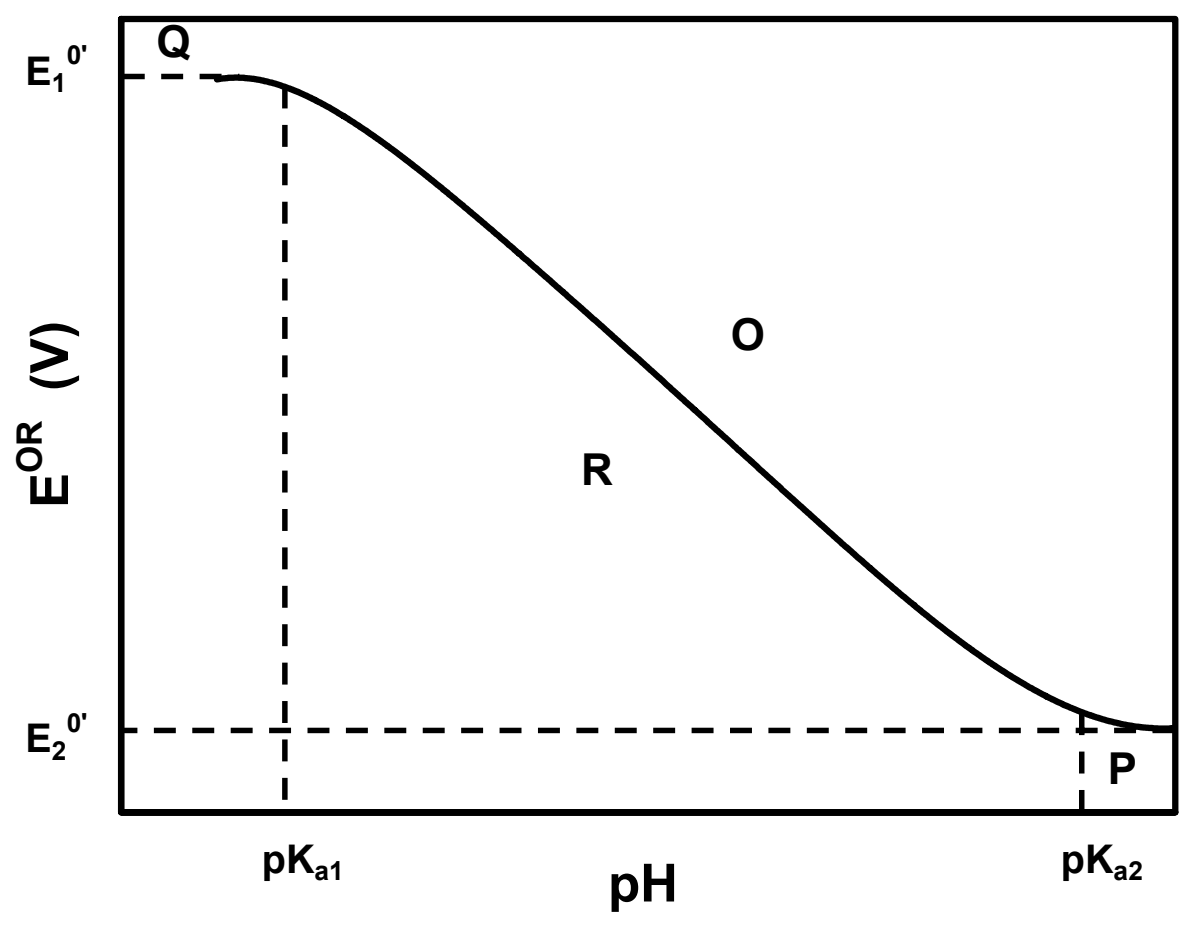

Figure 3.2. $\mathrm{E}^{\mathrm{OR}}$ vs. $\mathrm{pH}$ as predicted by Equation 3.2. (based on the four member square scheme, Figure 3.1). 
The overall rate of reaction is defined by summing the respective rates of the two separate half reactions $\mathrm{OP}$ and $\mathrm{QR}$.

$\frac{I}{F A}=k_{s_{1}}\left(\Gamma_{R} e^{\left(1-\alpha_{1}\right) f \eta_{1}}-\Gamma_{Q} e^{-\alpha_{1} f \eta_{1}}\right)+k_{s_{2}}\left(\Gamma_{P} e^{\left(1-\alpha_{2}\right) f \eta_{2}}-\Gamma_{O} e^{-\alpha_{2} f \eta_{2}}\right)$

where $\Gamma_{x}$ is the concentration of the respective species on the surface of the electrode in $\mathrm{mol} / \mathrm{cm}^{-2}, \eta_{1}=E-E_{1}^{0^{\prime}}$ and $\eta_{2}=E-E_{2}^{0^{\prime}}$ are the respective overpotentials for each redox pair.

Because proton transfer reactions are at equilibrium, it can be shown from Figure 3.1 that the acid dissociation constants are given by: $K_{a_{1}}=\frac{\left[H^{+}\right] \Gamma_{O}}{\Gamma_{Q}}$ and $K_{a_{2}}=\frac{\left[H^{+}\right] \Gamma_{p}}{\Gamma_{R}}$, which lead to: $\Gamma_{Q}=\frac{\left[H^{+}\right] \Gamma_{O}}{K_{a_{1}}}$ and

$\Gamma_{P}=\frac{K_{a_{2}} \Gamma_{R}}{\left[H^{+}\right]}$

Equation 3.4 can be simplified by substituting for $\Gamma_{\mathrm{Q}}$ and $\Gamma_{\mathrm{P}}$ using the two expressions in $3.5 \mathrm{a}$ and $3.5 \mathrm{~b}$ to give:

Rate $=k_{s_{1}}\left(\Gamma_{R} e^{\left(1-\alpha_{1}\right) f \eta_{1}}-\left(\frac{\left[H^{+}\right] \Gamma_{O}}{K_{a_{1}}}\right) e^{-\alpha_{1} f \eta_{1}}\right)+k_{s_{2}}\left(\left(\frac{K_{a_{2}} \Gamma_{R}}{\left[H^{+}\right]}\right) e^{\left(1-\alpha_{2}\right) f \eta_{2}}-\Gamma_{O} e^{-\alpha_{2} f \eta_{2}}\right)$

The total concentration of the oxidized species, $\Gamma_{O_{x}}$, is given by:

$\Gamma_{O_{x}}=\Gamma_{O}+\Gamma_{Q}=\Gamma_{O}\left(1+\frac{\left[H^{+}\right]}{K_{a_{1}}}\right)$

The terms containing $\Gamma_{\mathrm{O}}$ in Equation 3.6 relate to the apparent cathodic rate constant which can be defined as $-\frac{i}{F A \Gamma_{O_{x}}}$ leading to: 


$$
k_{c}^{O R} \text {,app. }=\left(1+\frac{\left[H^{+}\right]}{K_{a_{1}}}\right)^{-1}\left(k_{s_{2}} e^{-\alpha_{2} f \eta_{2}}+k_{s 1}\left(\frac{\left[H^{+}\right]}{K_{a_{1}}}\right) e^{-\alpha_{1} f \eta_{1}}\right)
$$

Likewise the anodic rate constant is

$$
k_{a \text { app. }}^{O R}=\left(1+\frac{K_{a_{2}}}{\left[H^{+}\right]}\right)^{-1}\left(k_{s_{1}} e^{\left(1-\alpha_{1}\right) f \eta_{1}}+k_{s_{2}}\left(\frac{K_{a_{2}}}{\left[H^{+}\right]}\right) e^{\left(1-\alpha_{2}\right) f \eta_{2}}\right)
$$

Each of the stepwise pathways OPR or OQR, shown in Figure 3.1, can be dominant under certain conditions. The relative contribution of each pathway can be expressed as a fraction of the overall process. From Equation 3.8, the percent contributed by OPR, can be obtained by dividing its contribution by the overall rate.

$$
\% \text { path } 1=\frac{100 \% k_{s_{1}}\left(\frac{\left[H^{+}\right]}{k_{a_{1}}}\right) e^{-\alpha_{1} f \eta_{1}}}{\left(k_{s_{1}}\left(\frac{\left[H^{+}\right]}{k_{a_{1}}}\right) e^{-\alpha_{1} f \eta_{1}}+k_{s_{2}} e^{-\alpha_{2} f \eta_{2}}\right)}
$$

The standard rate constant $\mathrm{k}_{\mathrm{s}}$ is defined under reversible conditions and is obtained by setting $\mathrm{E}=\mathrm{E}^{\mathrm{OR}}$. Under such conditions, the overpotential is defined with respect to reversible formal potential:

$$
\begin{aligned}
& \eta_{r_{1}}=E_{r}-E_{1}^{0^{\prime}}=\frac{2.3 R T}{F} \log \left(\frac{\left[H^{+}\right]+K_{a_{2}}}{\left[H^{+}\right]+K_{a_{1}}}\right) \\
& \eta_{r_{2}}=E_{r}-E_{2}^{0^{\prime}}=\frac{2.3 R T}{F} \log \left(\frac{k_{a_{2}}}{k_{a_{1}}}\right)\left(\frac{\left[H^{+}\right]+K_{a_{2}}}{\left[H^{+}\right]+K_{a_{1}}}\right)
\end{aligned}
$$

Substituting for $\eta_{1}$ and $\eta_{2}$ in Equations 3.8 and 3.9, yields: $\mathrm{k}_{\mathrm{s}, \mathrm{app}}=\mathrm{k}_{\mathrm{c}, \mathrm{app}}=\mathrm{k}_{\mathrm{a}, \text { app }}$. 


\subsection{Consequences of Laviron's Kinetic Predictions.}

The consequence of Laviron's first assumption is that, if the proton transfer step is fast and at equilibrium, the overall rate is determined by the electron transfer step. Figure 3.3 shows the variation of the standard rate constant with $\mathrm{pH}$. In the regions beyond the $\mathrm{pK}_{\mathrm{a}} \mathrm{s}$, the standard rate constant is independent of $\mathrm{pH}$. At $\mathrm{pH}$ below $\mathrm{pK}_{\mathrm{a} 1}$, the dominant redox species are the fully protonated forms (bottom of Figure 3.1) while at $\mathrm{pH}$ greater than $\mathrm{pK}_{\mathrm{a} 2}$, the deprotonated species (top of Figure 3.1) dominate. Under such conditions, pure electron transfer is the dominant process and $\mathrm{k}_{\mathrm{s}, \mathrm{app}}=\mathrm{k}_{\mathrm{s} 1 \text {,app. }}=\mathrm{k}_{\mathrm{s} 2 \text {,app. }}$. At intermediate pHs, $\left(\mathrm{pK}_{\mathrm{a} 1}<\mathrm{pH}<\mathrm{pK}_{\mathrm{a} 2}\right)$, the standard rate constant changes at a rate of about half an order of magnitude per $\mathrm{pH}$ unit, passing through a minimum at $\mathrm{pH}$ midway between the two $\mathrm{pK}_{\mathrm{a}} \mathrm{s}$. At the turning point, the standard rate constant can be several orders of magnitude lower than $\mathrm{k}_{\mathrm{s} 1}$ and $\mathrm{k}_{\mathrm{s} 2} .^{57}$

The cause for this behavior is due to non optimum electron transfer via either of the two pathways $\mathbf{O P R}$ or $\mathbf{O Q R} .{ }^{10}$ At $\mathrm{pH} 7$, for example, the reduction of $\mathbf{O}$ to $\mathbf{P}$ is more favorable (larger driving force) than the reduction of $\mathbf{Q}$ to $\mathbf{R}$ (smaller driving force). However, acid base equilibria heavily favors the deprotonated form $(\mathbf{Q})$. This opposing effect lowers the standard rate constant

The second assumption requires the transfer coefficient to be constant and equal to 0.5 at all potentials. Based on this assumption, the path of electron transfer between the dominant oxidized and reduced species depends only on $\mathrm{pH}$ and not on potential. Also, if cyclic voltammetry is used, the observed cyclic voltammograms (CVs) will be symmetrical at all scan rates over the entire $\mathrm{pH}$ range. This is because the free energy curves described in chapter one will be symmetrical when $\alpha=0.5$, and the barrier for 
oxidation and reduction is identical. Symmetric CVs lead to symmetric Tafel plots at all pHs. This was verified using simple electron transfer models such as ferrocene and $\operatorname{pyRu}\left(\mathrm{NH}_{3}\right)_{5}{ }^{2+} .{ }^{58}$

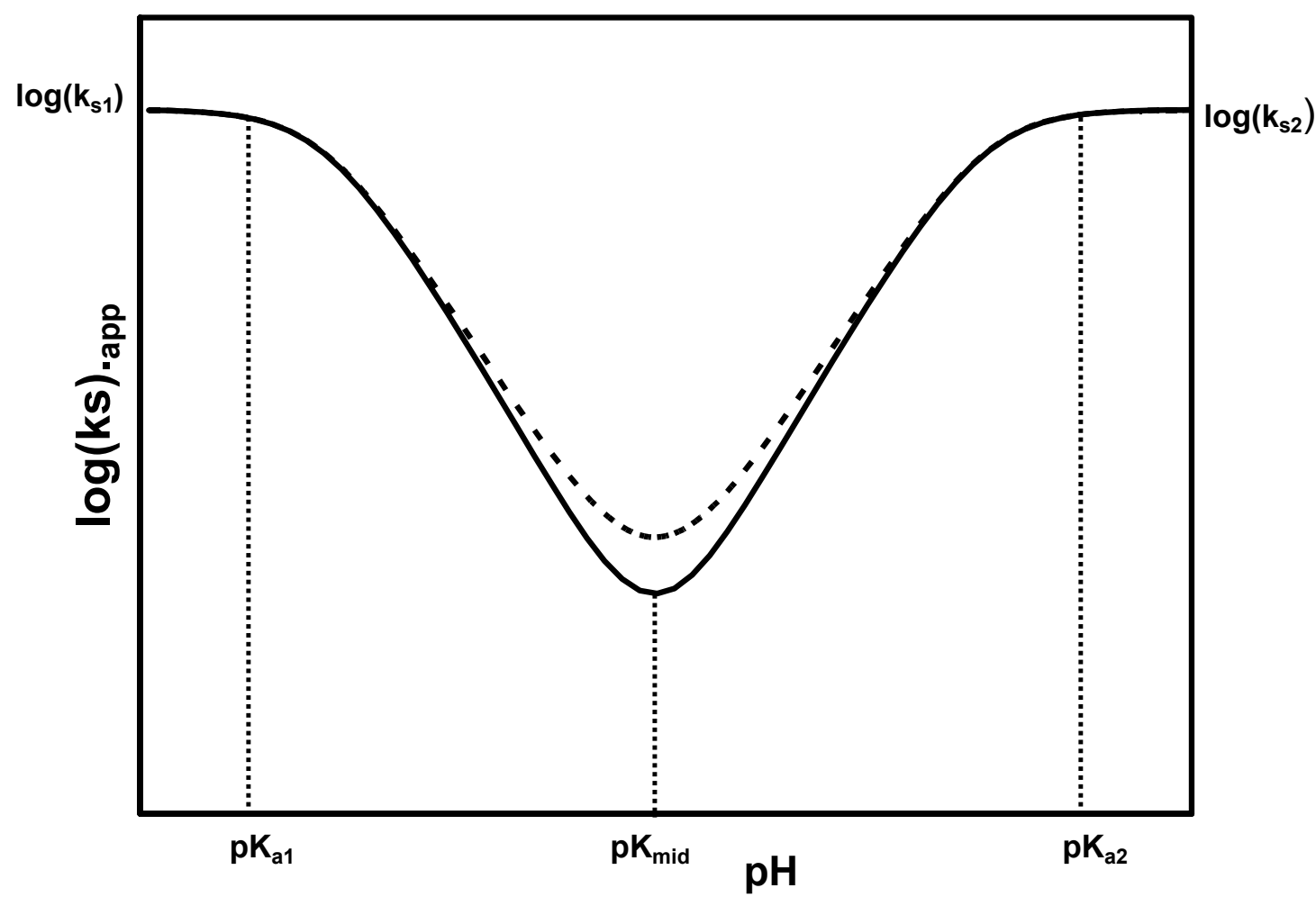

Figure 3.3. Theoretical predictions using both Laviron (dotted curve) and Finklea (solid curve) models.

\subsection{Finklea's Predictions.}

The Finklea model yields results that are qualitatively similar to Laviron's predictions. For example, the standard rate constant follows a similar pattern observed in Figure 3.3. However, the potential dependent transfer coefficient yields smaller values for the standard rate constant than Laviron's predictions (constant transfer coefficient). The consequence of this is that, at intermediate $\mathrm{pH}$, Finklea's model predicts a deeper 
minimum (solid curve in Figure 3.3) than Laviron's model. It is important to note here that Finklea's model only affect the kinetics and not the thermodynamic predictions made by Laviron.

The second consequence of a potential dependent transfer coefficient will be examined by looking at its variation with $\mathrm{pH}$. The transfer coefficient at zero overpotential, $\alpha(0)$ is a more useful parameter than $\alpha(\eta)$, because it is defined under standard (reversible) conditions. In order to evaluate $\alpha(0)$, the relationship between the apparent transfer coefficient and overpotential must be known. This relationship is described by rearranging Equations 1.10 and 1.11 (Chapter 1) to give:

$\alpha=-\frac{\ln \left(k_{c} / k_{s}\right)}{f \eta}$

$\alpha=\frac{1-\ln \left(k_{a} / k_{s}\right)}{f \eta}$

respectively.

A plot of $\alpha$ vs $\eta$ can be extrapolated to zero overpotential to yield $\alpha(0)$. A simulation by Finklea revealed that the potential dependence of the transfer coefficient could be accurately fit by a fifth order polynomial in $\eta .^{10}$

$\alpha=0.5+a \eta+b \eta^{3}+c \eta^{5}$

where the coefficients $\mathrm{a}, \mathrm{b}$ and $\mathrm{c}$ are dependent on the reorganization energy. For example, when $\lambda=0.7 \mathrm{eV}, \mathrm{a}=0.3082, \mathrm{~b}=-0.01926, \mathrm{c}=-0.009978$. Figure 3.4 is obtained by calculating $\alpha$ from the rate constants obtained from Equation 1.17 (Chapter 1). The transfer coefficient increases with overpotential, and the slope of the plot is always positive. Assuming that the reorganization energies are equal for the $\mathrm{Ox}$ and Red 
forms, $\alpha$ is only equal to 0.5 at the formal potential. Note that, for $\eta$ less than $\lambda$, the plot is nearly linear with a slope given by the "a" term.

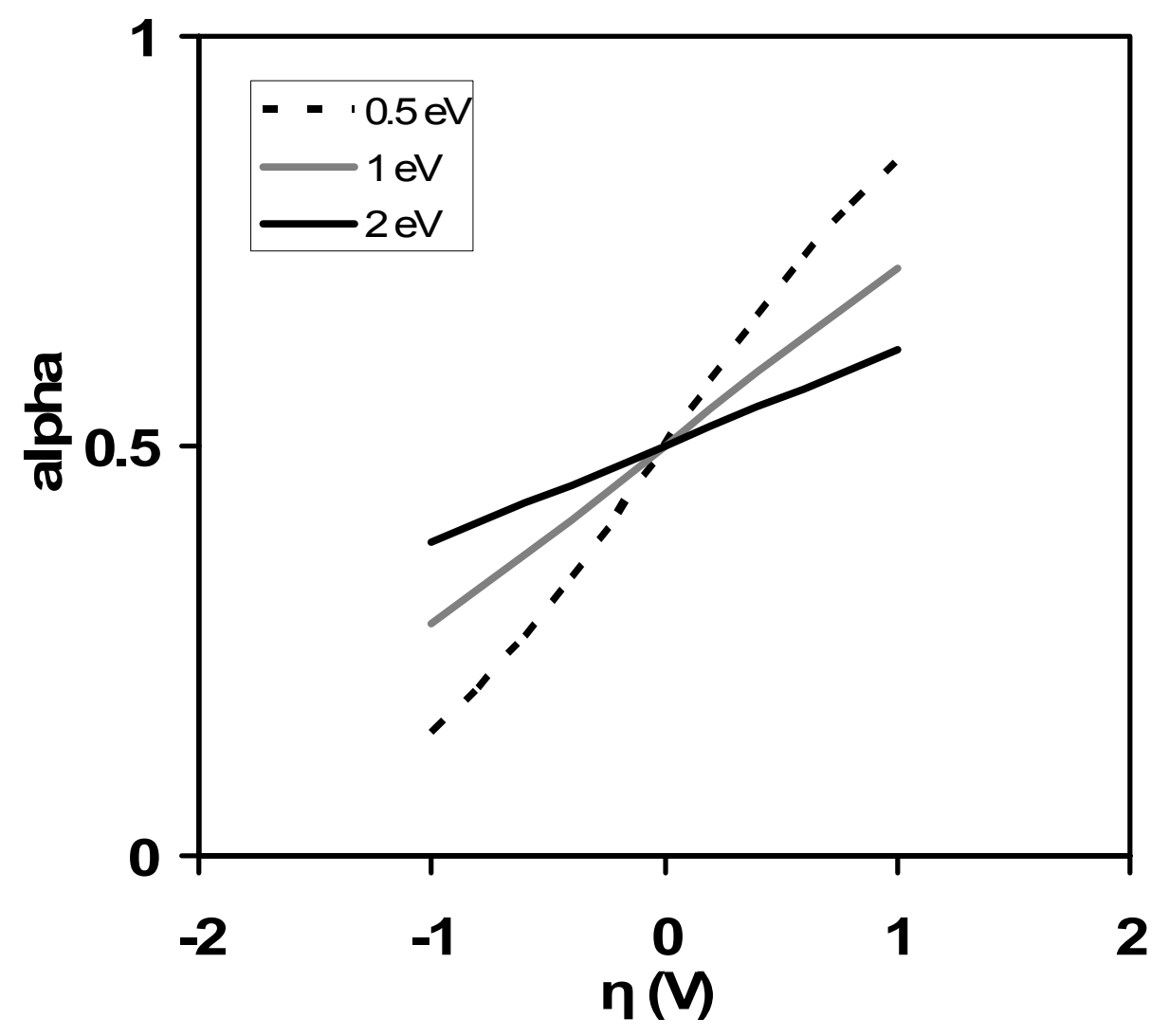

Figure 3.4. Potential dependence of transfer coefficient; effect of reorganization energy.

Figure 3.5 shows the variation of $\alpha(0)$ as a function of $\mathrm{pH}$. It is clear from this graph that $\alpha(0)$ is only equal to 0.5 at $\mathrm{pHs}$ lower than $\mathrm{pK}_{\mathrm{al}}$, at mid $\mathrm{pH}$ and at $\mathrm{pHs}$ greater than $\mathrm{pK}_{\mathrm{a} 2}$. At $\mathrm{pH}$ between $\mathrm{pK}_{\mathrm{a} 1}$ and mid $\mathrm{pH}, \alpha(0)$ is less than 0.5 while at $\mathrm{pH}$ between mid $\mathrm{pH}$ and $\mathrm{pK}_{\mathrm{a} 2}, \alpha(0)$ is greater than 0.5 . The consequence of this behavior is that the CVs are asymmetrical. At $\mathrm{pH}$ closer to $\mathrm{pK}_{\mathrm{a} 2}$, anodic $\mathrm{CVs}$ will be broader than cathodic CVs and likewise, at $\mathrm{pH}$ closer to $\mathrm{pK}_{\mathrm{a} 1}$, cathodic $\mathrm{CVs}$ will be broader than anodic CVs. Asymmetric CVs lead to asymmetry in Tafel plots. For example, at $\mathrm{pH}$ close to $\mathrm{pK}_{\mathrm{a} 2}$, the 
broadened anodic CVs give rise to a Tafel plot with a less steep anodic branch than the cathodic branch. A similar argument can be applied to behavior at $\mathrm{pH}$ close to $\mathrm{pK}_{\mathrm{a} 1}$.

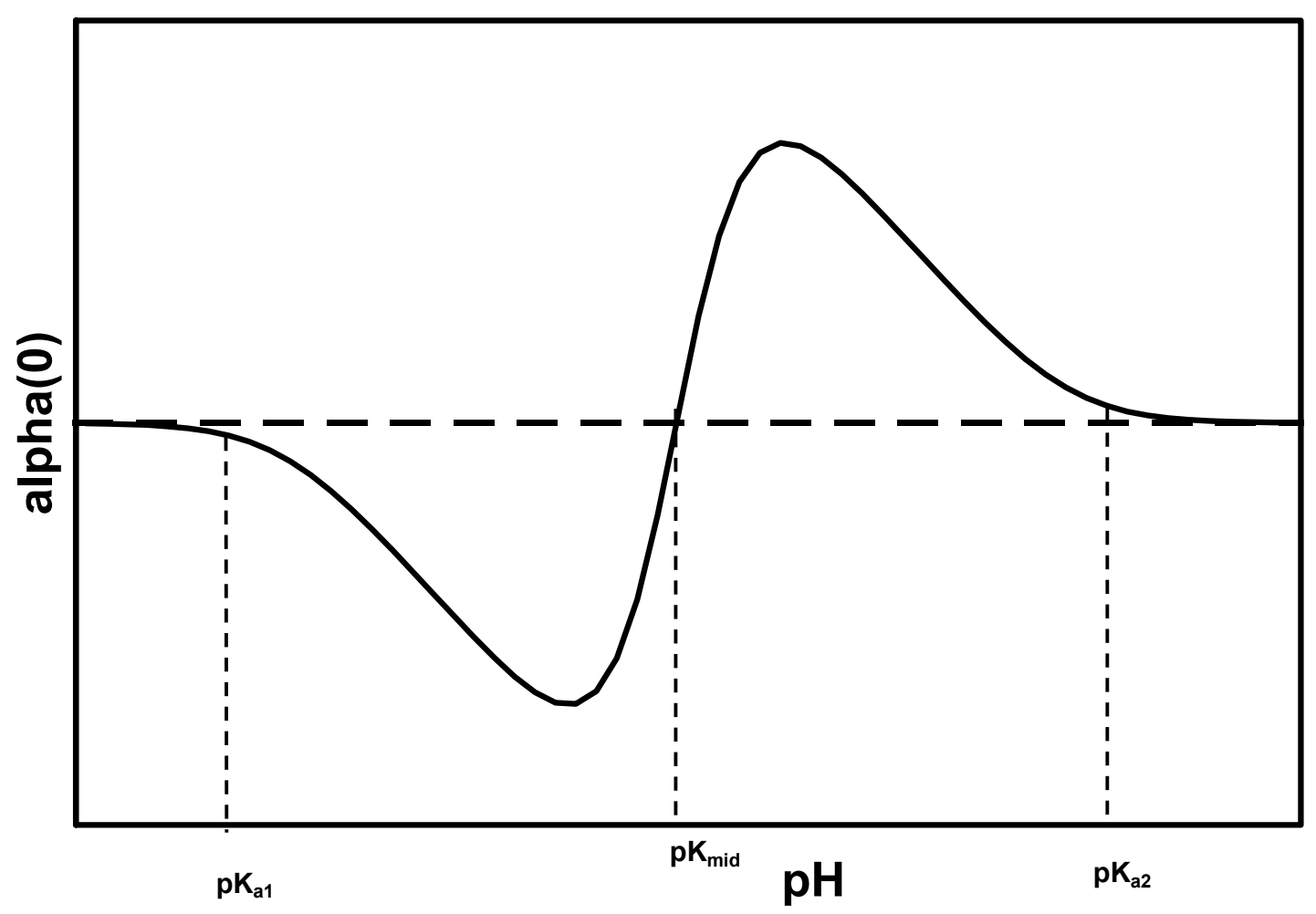

Figure 3.5. Theoretical predictions using Finklea model; variation of the transfer coefficient with $\mathrm{pH}$.

Finklea's model predicts that the path of electron transfer depends on both the $\mathrm{pH}$ and the potential. For example, the path for electron transfer can switch from OPR to $\mathrm{OQR}$ (Figure 3.1.) as the potential changes.

In this study, predictions from Laviron and Finklea models will be further evaluated by using a model osmium aquo complex, $\left.\mathrm{Os}(\mathrm{bpy})_{2}(\mathrm{py})\left(\mathrm{H}_{2} \mathrm{O}\right)\right]^{2+}$ (bpy is 2,2'bipyridine, py is 4-aminomethylpyridine), attached to long chain self-assembled monolayers on an electrode. Details about this compound are given in chapter 4 . 


\section{CHAPTER 4: THE OSMIUM SYSTEM}

\subsection{Synthesis of osmium polypyridyl complexes}

The motivation for this synthetic pathway was the need for a model system that can undergo a 1e,1H proton coupled electron transfer. Since organometallic compounds can be easily synthesized with a high degree of control over their electrochemical and spectroscopic properties, they can be employed for such a study. A suitable candidate would be a metal whose inner coordination sphere is composed of stable non-ionizable ligands. One of the coordination sites should have a ligand that is able to undergo proton transfer depending on the $\mathrm{pH}$ of the contacting solution. Because of their variable oxidation states, transition metals would be suitable candidates for electron transfer model systems. To simplify the chemistry involved, the ionizable ligand should be able to lose or gain a single proton depending on the $\mathrm{pH}$ of the solution. Multi-proton transfer sites require complex theoretical treatments that are beyond the scope of this study.

Complexes of osmium and ruthenium have attracted a lot of interest for several decades. ${ }^{61-67}$ Of particular interest are the polypyridyl complexes of these metals. Polypyridyl complexes of osmium and ruthenium form stable compounds which exhibit interesting photochemical and electrochemical properties. This stability emanates from the fact that electron transfer processes occur from $\mathrm{d} \pi$ levels rather than the sigma framework of these complexes. ${ }^{67}$ Meyer and coworkers have done an extensive and systematic study of these compounds to elucidate their electrochemical and spectroscopic properties. ${ }^{62-64}$ One of the many complexes they studied was $\left[\mathrm{Os}(\mathrm{tpy})(\mathrm{bpy})\left(\mathrm{OH}_{2}\right)\right]^{2+}$ Figure 4.1 shows a Pourbaix diagram for this compound, which provides details of the dominant species at different $\mathrm{pHs}$ and corresponding formal potentials. 


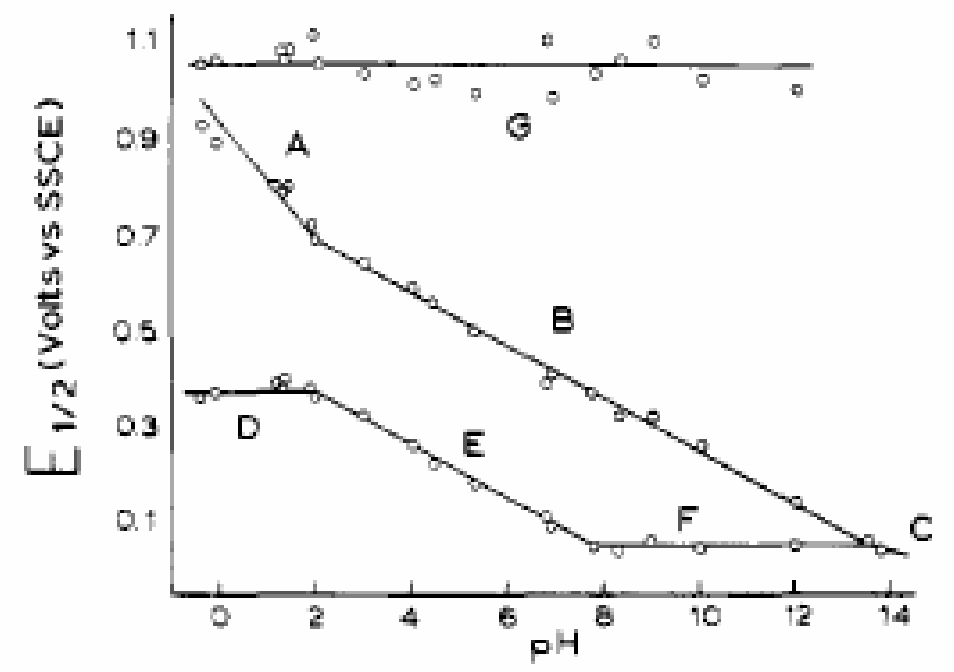

Figure 4.1. Pourbaix diagram for the Os(V/IV), Os(IV/III) and Os(III/II) redox couples of $\left[\mathrm{Os}(\mathrm{tpy})(\mathrm{bpy})\left(\mathrm{OH}_{2}\right)\right]^{2+}$. Data were collected in $0.1 \mathrm{M}$ aqueous buffer on a glassy-carbon working electrode. (A): $\left[\mathrm{Os}^{\mathrm{IV}}=\mathrm{O}\right]^{2+} /\left[\mathrm{Os}^{\mathrm{III}}-\mathrm{OH}_{2}\right]^{3+} ;(\mathrm{B}):\left[\mathrm{Os}^{\mathrm{IV}}=\mathrm{O}\right]^{2+} /\left[\mathrm{Os}^{\mathrm{III}}-\mathrm{OH}\right]^{2+} ;(\mathrm{C})$ : $\left[\mathrm{Os}^{\mathrm{IV}}=\mathrm{O}\right]^{2+} /\left[\mathrm{Os}{ }^{\mathrm{II}}-\mathrm{OH}\right]^{+} ;(\mathrm{D}):\left[\mathrm{Os}^{\mathrm{III}}-\mathrm{OH}_{2}\right]^{3+} /\left[\mathrm{Os}^{\mathrm{II}}-\mathrm{OH}_{2}\right]^{2+} ;(\mathrm{E}):\left[\mathrm{Os}{ }^{\mathrm{III}}-\mathrm{OH}\right]^{2+} /\left[\mathrm{Os}^{\mathrm{II}}-\mathrm{OH}_{2}\right]^{2+}$; (F): $\left[\mathrm{Os}{ }^{\mathrm{III}}-\mathrm{OH}\right]^{2+} /\left[\mathrm{OS}{ }^{\mathrm{II}}-\mathrm{OH}\right]^{+} ;(\mathrm{G}):\left[\mathrm{Os}^{\mathrm{V}}=\mathrm{O}\right]^{3+} /\left[\mathrm{Os}^{\mathrm{IV}}=\mathrm{O}\right]^{2+}{ }^{62}$ This diagram was taken from reference number 62 .

Osmium can undergo electron transfer between the +2 and +3 states. Although this compound can be further oxidized, up to +6 , only the first redox wave will be relevant to this study. For the $\mathrm{Os}^{\mathrm{II} / \mathrm{II}}$ oxidation state, the hydrogens on the aquo ligand are ionizable at $\mathrm{pHs}$ above $\mathrm{pK}_{\mathrm{a} 1}$ of this compound (approximately 2); therefore, this complex can undergo proton transfer between the aquo ligand and the aqueous solvent. Another important feature of this compound is that its two $\mathrm{pK}_{\mathrm{a}} \mathrm{s}\left(\mathrm{pK}_{\mathrm{a} 1}=2, \mathrm{pK}_{\mathrm{a} 2}=8\right)$ are well separated to cover reasonable $\mathrm{pH}$ ranges which are readily accessible in aqueous solutions. The second wave $(+3 /+4)$ starts merging with the $+2 /+3$ wave at higher $\mathrm{pHs}$, above $\mathrm{pK}_{\mathrm{a} 2}$. Moreover, the corresponding formal potentials $E_{1}^{0}$ and $E_{2}^{0}$ are well 
separated and lie within the potential window of the gold electrode. The formal potentials for $\mathrm{Os}^{\mathrm{IIIIII}}$ and $\mathrm{Os}^{\mathrm{III/IV}}$ are separated by a potential of at least $0.2 \mathrm{~V}$ at all $\mathrm{pHs}$, making it possible for PCET studies of the $\mathrm{Os}^{\mathrm{II} / \mathrm{III}}$ wave to be carried out within a reasonable potential window.

However, carrying out PCET studies at an electrode with the osmium complex in homogeneous solution phase poses a great challenge, especially with currently available technology. One of the major challenges includes measuring rates of electron transfer which can reach up to $10^{5} \mathrm{~cm} / \mathrm{s}$, which requires microelectrodes and specialized instrumentation, such as scanning electrochemical microscopy. ${ }^{68}$ The second challenge has to do with the nature of the electrode itself. Because of its heterogeneous nature, the concentration, orientation and structure of the redox molecule at or near the electrode can be significantly different from the bulk due to mass transfer and double layer effects. These problems make it extremely difficult to obtain kinetic information.

As already discussed, recent studies have shown that the rates of electron transfer can be greatly slowed down by using self-assembled monolayers. ${ }^{15}$ Increasing the chain length of the monolayer can reduce the rate of electron transfer to values that are measurable with current equipment. The redox molecule is tethered on one end of the SAM chain and kept at a constant distance away from the electrode. The tether can be carefully chosen to take advantage of the established coupling chemistry such as amide bond formation. In choosing a suitable tethering molecule, it is important to maintain the structure and electrostatic nature of the complex itself in order to preserve its thermodynamic properties. A variety of different molecules can be used as tethers 
depending on availability of synthetic methods to introduce these molecules into the coordination sphere of the redox molecule.

Based on this background, a model system whose electrochemical properties are $\mathrm{pH}$ dependent and accessible for the entire $\mathrm{pH}$ scale is considered. The $\left[\mathrm{Os}(\mathrm{bpy})_{2}(\mathrm{py})\left(\mathrm{OH}_{2}\right)\right]^{2+}$ was found to be a suitable model. This complex is a slight modification from Meyer's compound discussed above. In this molecule, the terpyridine ligand has been replaced by 4-Aminomethylpyridine (py) and a second bipyridine molecule. A tether has been introduced into this molecule that will allow it to be coupled to a self-assembled monolayer through the free amine group on 4-Aminomethylpyridine. These changes, however, should not significantly alter the thermodynamic properties of this molecule with respect to the $\left[\mathrm{Os}(\mathrm{tpy})(\mathrm{bpy}) \mathrm{OH}_{2}\right]^{2+}$ molecule. ${ }^{45}$ By using a SAM with a carboxylic acid terminal group, this compound can be coupled to the electrode by forming a peptide bond between the $-\mathrm{COOH}$ group on the $\mathrm{SAM}$ and the $-\mathrm{NH}_{2}$ group on 4-Aminomethylpyridine using established coupling methods. ${ }^{69-71}$ The fact that this molecule can now be attached to a SAM means that the kinetics of electron transfer can be significantly slowed to values that are measurable with currently available equipment. These attributes allow us to do a systematic study to obtain both thermodynamic and kinetic information for this system.

Described in the following sections are details of pathways used to synthesize $\left[\mathrm{Os}(\mathrm{bpy})_{2}(\mathrm{py})\left(\mathrm{OH}_{2}\right)\right]^{2+}$ as well as methods used for coupling the redox molecule to SelfAssembled Monolayers on an electrode. 


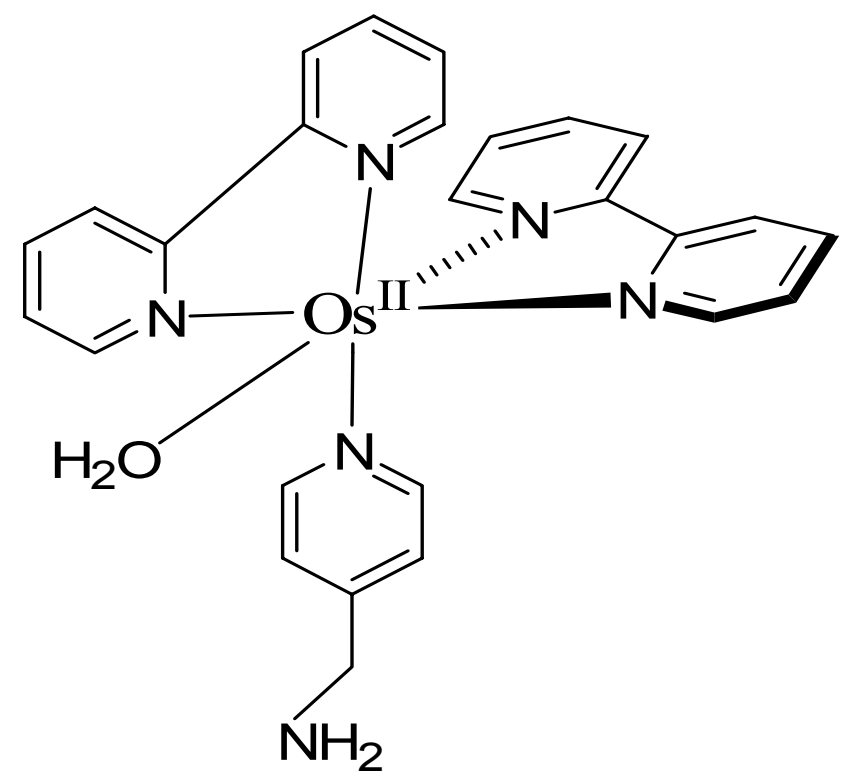

Figure 4.2. Structure of $\left[\mathrm{Os}(\mathrm{bpy})_{2}(\mathrm{py})\left(\mathrm{OH}_{2}\right)\right]^{2+},(\mathrm{bpy}=$ bipyridine, py $=4$-Aminomethylpyridine). 


\subsubsection{Synthesis of $\left[\mathrm{Os}(\mathrm{bpy})_{2}(\mathrm{py})\left(\mathrm{OH}_{2}\right)\right]^{2+}$}

The synthesis of this molecule was achieved via several synthetic steps using commercially available starting materials. Each of these steps or stages will be discussed separately. Most of the stages were based on established methods. At each stage, either $\mathrm{UV} / \mathrm{Vis}$ spectra, electrochemical analysis, or both studies were done in the electrolytes indicated to ascertain the authenticity and purity of the product before proceeding to the next step. 


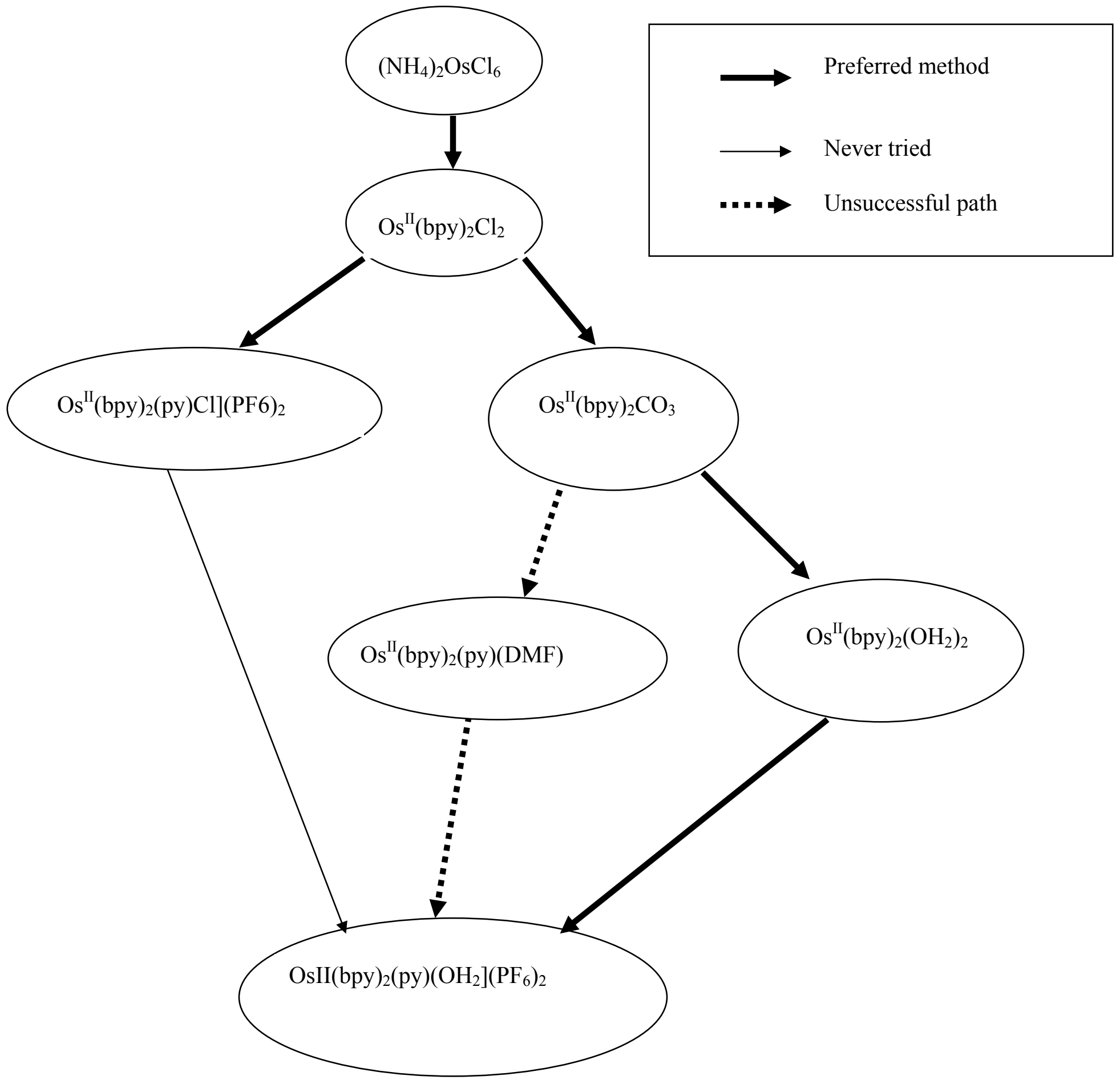

Figure 4.3. Scheme of general synthetic strategy for $\left[\mathrm{Os}(\mathrm{bpy})_{2}(\mathrm{py})\left(\mathrm{OH}_{2}\right)\right]^{2+}$ 


\subsubsection{STAGE (I): $\left(\mathrm{NH}_{4}\right)_{2} \mathrm{OsCl}_{6} \rightarrow \mathrm{Os}(\mathrm{bpy})_{2} \mathrm{Cl}_{2}$}

The synthesis of the cis-dichlorobis(bipyridine)osmium(II) complex, $\mathrm{Os}(\mathrm{bpy})_{2} \mathrm{Cl}_{2}$ is well established and is based on the original report by Buckingham et al. ${ }^{61}$ Since Buckingham's report, several other reports followed, and, in particular, T. J. Meyer et al have done extensive work on this compound. ${ }^{62-64}$ The synthetic method used here was based on a method reported by Meyer ${ }^{64}$ and adopted by Haddox. ${ }^{45}$ Approximately $500 \mathrm{mg}$ (1.14 mmol) of $\left(\mathrm{NH}_{4}\right)_{2} \mathrm{OsCl}_{6}$ and $350 \mathrm{mg}$ (2.24 mmol ) of 2,2' dipyridyl were heated to reflux in $10 \mathrm{ml}$ of deoxygenated DMF in a $50 \mathrm{ml}$ round bottom flask (rbf). The resulting mixture was refluxed under a blanket of nitrogen for one hour. The solution was cooled to room temperature. Since the crude mixture contained both cis-Os ${ }^{\mathrm{II}}(\mathrm{bpy})_{2} \mathrm{Cl}_{2}$ and cis$\left[\mathrm{Os}^{\mathrm{III}}(\mathrm{bpy})_{2} \mathrm{Cl}_{2}\right]^{+}$, saturated sodium dithionite solution was added to reduce the metal center from +3 to +2 . This step was followed by cooling in an ice bath. The resulting purple black precipitate was washed thoroughly using plenty of distilled de-ionized water to remove $\left[\mathrm{Os}(\mathrm{bpy})_{3}\right]^{2+}$. Finally the product was washed with copious amounts of diethyl ether followed by drying overnight in a dessicator. Typical yield was about $65 \%$. 


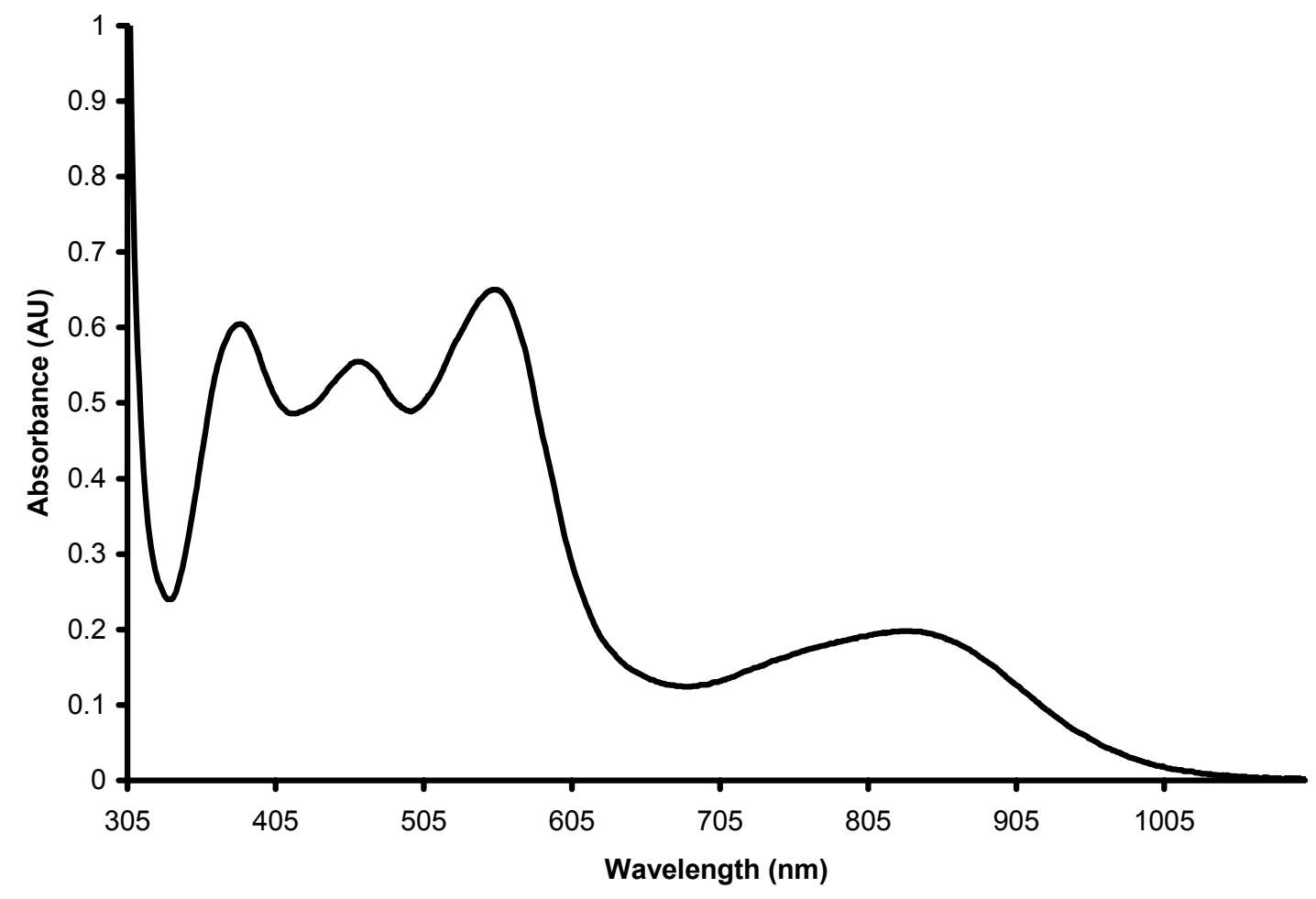

Figure 4.4. UV spectrum of $\mathrm{Os}(\mathrm{bpy})_{2} \mathrm{Cl}_{2}$ in $\mathrm{CH}_{3} \mathrm{CN}$.

\begin{tabular}{|c|c|c|}
\hline $\begin{array}{l}\text { Experimental } \lambda_{\max } \\
(\mathrm{nm})\end{array}$ & Literature $\lambda_{\max }(\mathrm{nm})$ & $\begin{array}{l}\text { Molar absorptivity } \\
\left(\mathrm{M}^{-1} \mathrm{Cm}^{-1}\right)\end{array}$ \\
\hline 383 & 382 & 10500 \\
\hline 460 & 467 & 9500 \\
\hline 552 & 562 & 10500 \\
\hline 830 & 820 & 2900 \\
\hline
\end{tabular}

Table 4.1. Experimental and literature UV data for Os(bpy) ${ }_{2} \mathrm{Cl}_{2}{ }^{64}$ 


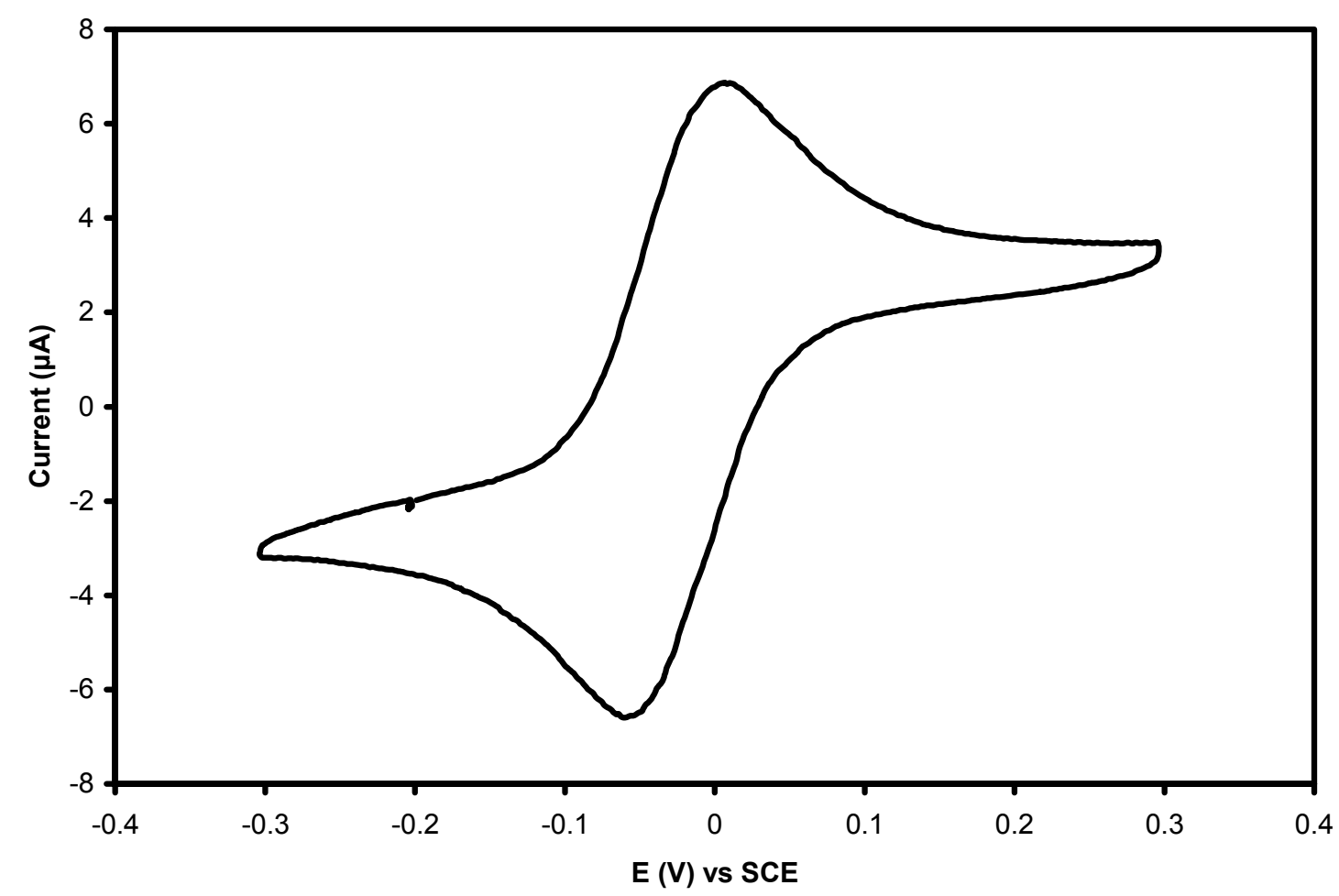

Figure 4.5. Cyclic Voltammogram $(0.1 \mathrm{~V} / \mathrm{s})$ of $\mathrm{Os}(\mathrm{bpy})_{2} \mathrm{Cl}_{2}\left(8 \times 10^{-4} \mathrm{M}\right)$, in $0.1 \mathrm{M}$ tetramethylammonium tetrafluoroborate $/ \mathrm{CH}_{3} \mathrm{CN}$ using a bare gold working electrode $\mathrm{E}^{\mathrm{o}^{\prime}}=-0.03 \mathrm{~V}$ vs SCE.

A reasonable yield was obtained for this product and the voltammogram shows a very pure product. The experimental formal potential (-0.03V vs SCE) agrees very well with the value reported in literature (-0.04V vs SSCE) ${ }^{63} \mathrm{UV}$ analysis shows all four peaks reported in literature and there is reasonable agreement between the peak positions.

\subsubsection{STAGE (II): $\left.\mathrm{Os(bpy)})_{2} \mathrm{Cl}_{2} \longrightarrow \mathrm{Os(bpy}\right)_{2} \mathrm{CO}_{3}$}

Os(bpy $)_{2} \mathrm{CO}_{3}$ was synthesized following Meyer's method ${ }^{45,63,64}$. In a typical synthesis, $(0.17 \mathrm{mmol})$ of $\mathrm{Os}(\mathrm{bpy})_{2} \mathrm{Cl}_{2}$ was added to $15 \mathrm{ml}$ of deoxygenated distilled deionized water in a $50 \mathrm{ml} \mathrm{rbf.,} \mathrm{sodium} \mathrm{carbonate}(2 \mathrm{~g})$ was added, followed by refluxing 
the mixture under an inert atmosphere of nitrogen for two hours. The solution was allowed to cool to room temperature before another $2 \mathrm{~g}$ portion of sodium carbonate was added, followed by an additional two hours of reflux. This procedure was repeated one more time. Upon cooling, a black microcrystalline product was collected. The product was rinsed thoroughly with distilled de-ionized water whose $\mathrm{pH}$ had been adjusted above pH 9. According to Meyer and coworkers ${ }^{64}$, rinsing was supposed to be continued until the product turned to a reddish brown color. This condition was not achieved even after rinsing with over $800 \mathrm{ml}$ of distilled de-ionized water. A dark purple/ red product was obtained instead. However, purity of the product as determined by coulometry was high 90\%. Analysis of the product by Cyclic Voltammetry was limited by the lability of the carbonate ligand. Upon dissolution of $\mathrm{Os}(\mathrm{bpy})_{2} \mathrm{CO}_{3}$ in acidic solutions, a bis(aqua) complex is formed through protonation and subsequent loss of the carbonate ligand as carbon dioxide. $^{63}$

$\mathrm{Os}(\mathrm{bpy})_{2} \mathrm{CO}_{3}+2 \mathrm{H}^{+}+\mathrm{H}_{2} \mathrm{O} \rightarrow\left[\mathrm{Os}(\mathrm{bpy})_{2}\left(\mathrm{OH}_{2}\right)_{2}\right]^{2+}+\mathrm{CO}_{2}$

The bis-aquo species, however, has a characteristic cyclic voltammogram. The experimental cyclic voltammogram (Figure. 4.7) was compared with literature, Figure. 4.6, as a way of product confirmation and checking purity. All three waves were observed by cyclic voltammetry. A UV spectrum of the carbonato complex (Figure. 4.8) was obtained in acetonitrile and compared with literature. Both UV and cyclic voltammetry confirmed that the product was pure. Typical yield was $60 \%$. 


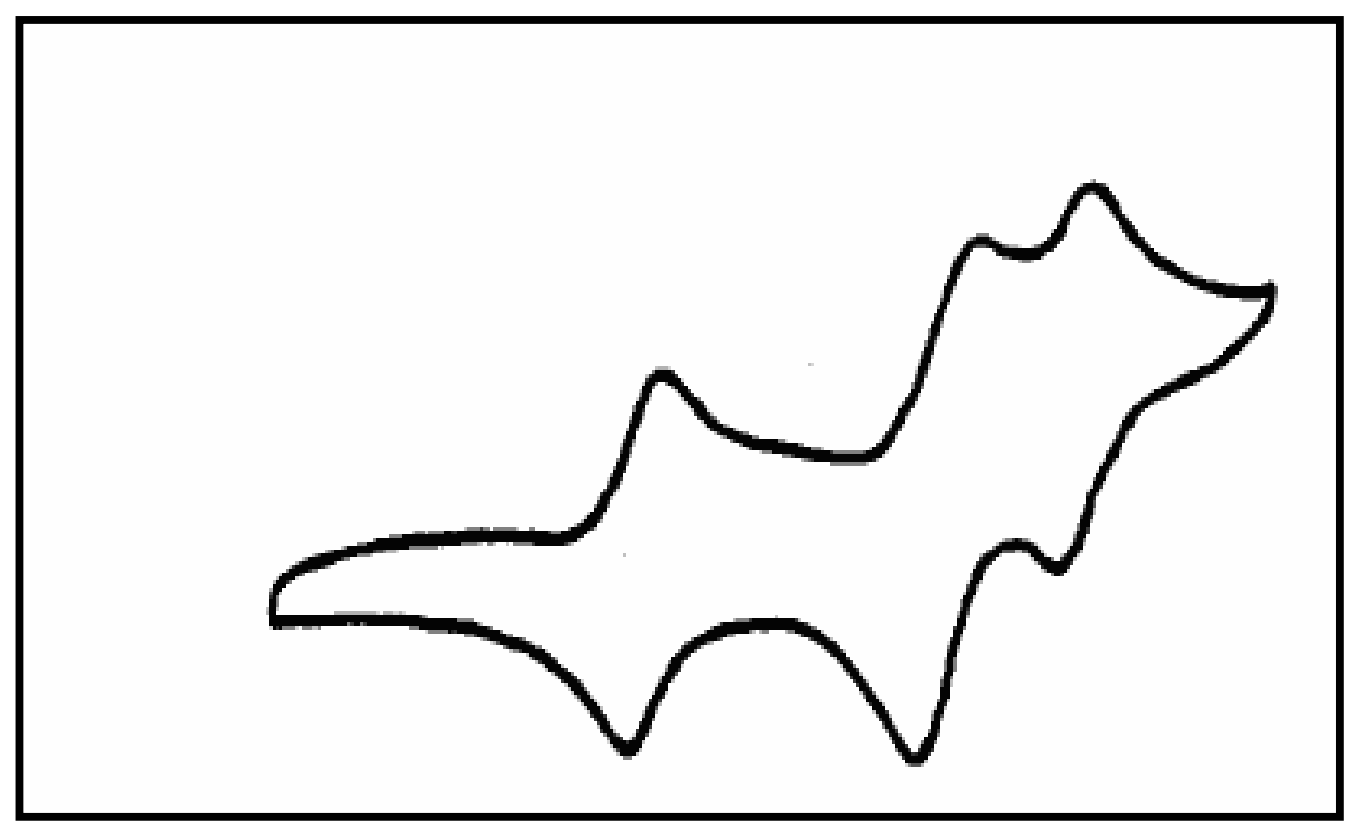

Figure 4.6. Cyclic Voltammogram of $\left[\mathrm{Os}(\mathrm{bpy})_{2}\left(\mathrm{OH}_{2}\right)_{2}\right]^{2+}$ from $\mathrm{Os}(\mathrm{bpy})_{2} \mathrm{CO}_{3}$ at $\mathrm{pH} 1.4{ }^{63}$ This Figure was obtained from reference number 63.

Figure 4.6 is a literature $\mathrm{CV}$ for $\left[\mathrm{Os}(\mathrm{bpy})_{2}\left(\mathrm{OH}_{2}\right)_{2}\right]^{2+}$ from $\mathrm{Os}(\mathrm{bpy})_{2} \mathrm{CO}_{3} \cdot{ }^{63}$ Three waves were observed. The first wave near $0.16 \mathrm{~V}$ vs. SSCE was attributed to a one electron process from coulometric studies. This wave was assigned to $\mathrm{Os}^{(\mathrm{III})} / \mathrm{Os}{ }^{(\mathrm{II})}$ couple. The second wave near $0.61 \mathrm{~V}$ vs. SSCE was believed to be a two electron process and was assigned to a Os ${ }^{(\mathrm{V})} / \mathrm{Os}^{(\mathrm{III})}$ couple. The final wave at around $0.81 \mathrm{~V}$ vs. SSCE was another one electron process attributed to the $\mathrm{O}^{(\mathrm{VI})} / \mathrm{Os}^{(\mathrm{V})}$ couple. All three waves were observed in this study, Figure 4.7. 


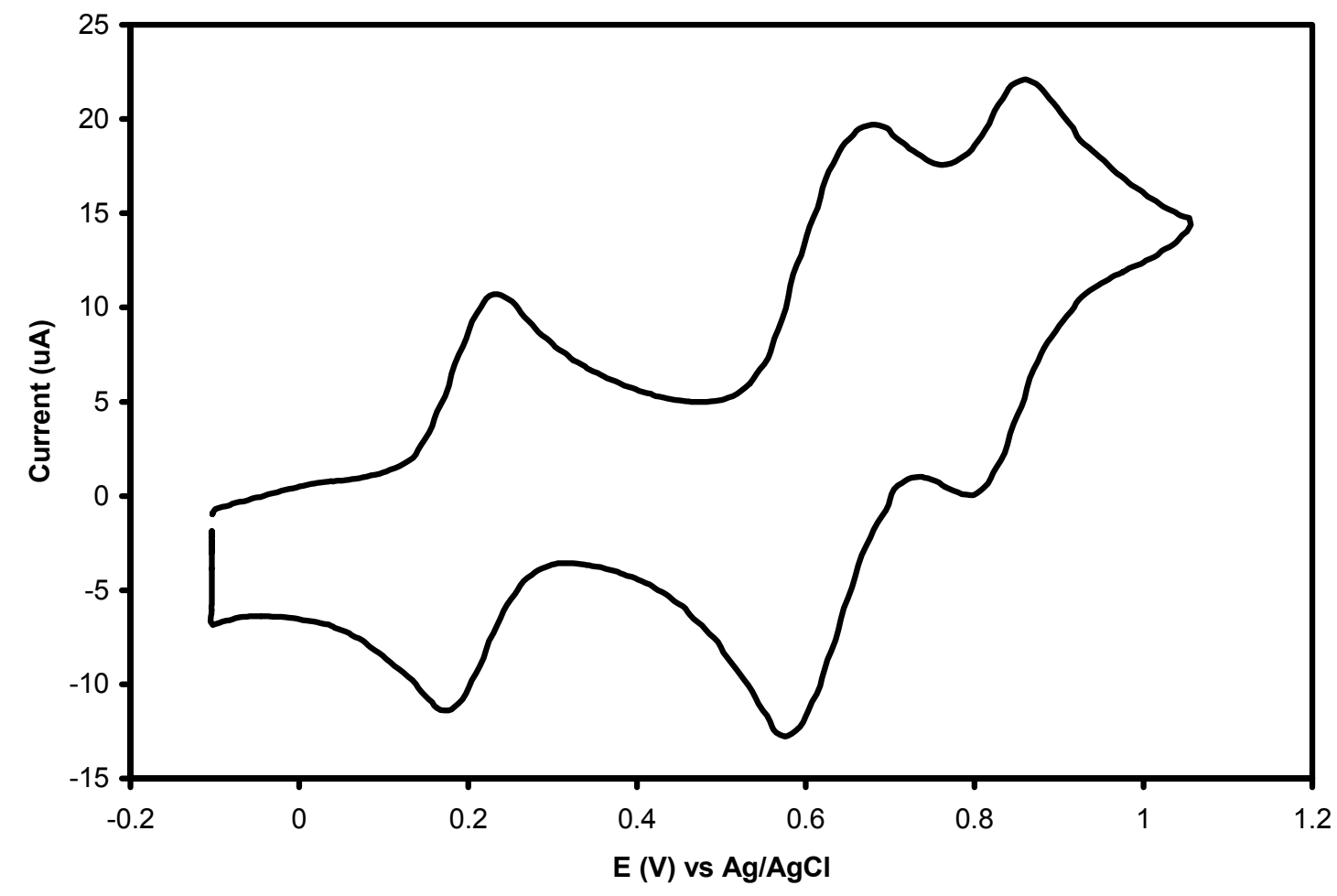

Figure 4.7. Cyclic Voltammogram $(0.1 \mathrm{~V} / \mathrm{s})$ of cis-Os $(\mathrm{bpy})_{2}\left(\mathrm{OH}_{2}\right)_{2}$ from $\mathrm{Os}(\mathrm{bpy})_{2} \mathrm{CO}_{3}$ in $0.1 \mathrm{M} \mathrm{HClO}_{4}$ 


\begin{tabular}{|c|l|c|}
\hline $\begin{array}{l}\text { Formal Potential } \mathrm{E}^{0} \\
\text { (Volts) vs. } \mathrm{Ag} / \mathrm{AgCl}\end{array}$ & Assignment & $\begin{array}{c}\text { Literature Value } \\
\text { (Volts) vs. SSCE }\end{array}$ \\
\hline 0.20 & Os(II)/Os(III) & 0.16 \\
\hline 0.62 & Os(III)/Os(V) & 0.61 \\
\hline 0.83 & Os(V)/Os(VI) & 0.81 \\
\hline
\end{tabular}

Table 4.2. Formal potential data for $\left[\mathrm{Os}(\mathrm{bpy})_{2}\left(\mathrm{OH}_{2}\right)\right]^{2+}$ from $\mathrm{Os}(\mathrm{bpy})_{2} \mathrm{CO}_{3}{ }^{63}$ 


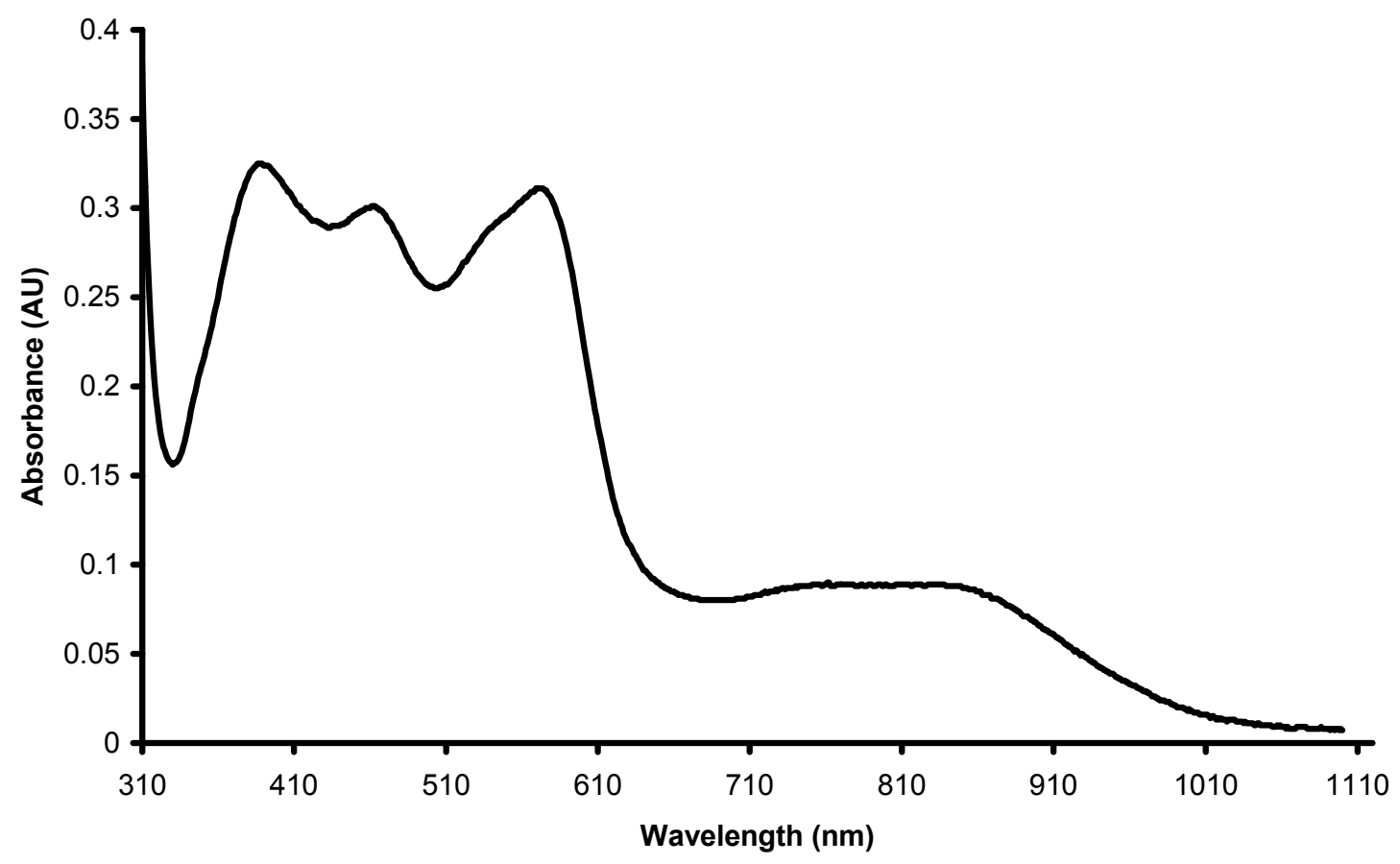

Figure 4.8. UV spectrum of $\mathrm{Os}(\mathrm{bpy})_{2} \mathrm{CO}_{3}$ in $\mathrm{CH}_{3} \mathrm{CN}$

\begin{tabular}{|c|c|c|}
\hline Experimental $\lambda_{\max }(\mathrm{nm})$ & Literature $\lambda_{\max }(\mathrm{nm})$ & $\begin{array}{c}\text { Molar absorptivity } \\
\left(M^{-1} \mathrm{~cm}^{-1}\right)\end{array}$ \\
\hline 389 & 389 & 10500 \\
\hline 463 & 476 & 9800 \\
\hline 574 & 581 & 10000 \\
\hline 830 & 820 & 3000 \\
\hline
\end{tabular}

Table 4.3. Experimental and literature UV data for $\mathrm{Os}(\mathrm{bpy})_{2} \mathrm{CO}_{3}{ }^{64}$ 
Electrochemical data for both the cis-dichloro and the carbonato complex compare well with literature values. UV analysis also shows a reasonably good agreement between experimental and literature data. All the peaks reported in literature were observed for both complexes.

\subsubsection{STAGE (III):Os(bpy $)_{2} \mathrm{CO}_{3} \longrightarrow\left[\mathrm{Os}(\mathrm{bpy})_{2}(\mathrm{OH})_{2}\right]^{2+} \rightarrow\left[\mathrm{Os}(\mathrm{bpy})_{2}(\mathrm{py})\left(\mathrm{OH}_{2}\right)\right]\left(\mathrm{PF}_{6}\right)_{2}$.}

This synthetic method was developed in Dr Finklea's lab. One of the methods reported by Meyer for the synthesis of $\left[\mathrm{Os}(\mathrm{tpy})(\mathrm{bpy})\left(\mathrm{OH}_{2}\right)\right]^{2+}$ involved using the $[\mathrm{Os}(\mathrm{tpy})(\mathrm{bpy}) \mathrm{OsCl}]^{+}$as the precursor. ${ }^{64}$ In this method, trifluoromethanesulfonic acid was used to displace the chloride ligand as $\mathrm{HCl}$ gas. This was then followed by aquating the resulting product and reducing it with zinc amalgam to form the final product, $\left[(\text { tpy })(\text { bpy }) \mathrm{Os}\left(\mathrm{OH}_{2}\right)\right]^{2+}$. Although this method was successful, the authors reported some difficulties in the synthetic method, largely due to the high affinity of the Os(II) metal center for the chloride ligand as well as its inertness towards substitution. Also, there were problems with dimerization resulting in the formation of osmium oxo-bridges and mixed products.

In the Meyer method, $0.2 \mathrm{~g}$ of $[(\mathrm{tpy})(\mathrm{bpy}) \mathrm{OsCl}]\left(\mathrm{PF}_{6}\right)(0.26 \mathrm{mmol})$ were added to $3 \mathrm{ml}$ of concentrated trifluoromethanesulfonic acid. The resulting mixture was heated to $110{ }^{\circ} \mathrm{C}$ under argon, for one hour. The reaction mixture was cooled to room temperature followed by addition of $5 \mathrm{ml}$ of distilled water and zinc amalgam as a reductant. After zinc amalgam was removed, $0.5 \mathrm{ml}$ of $\mathrm{NH}_{4} \mathrm{PF}_{6}$ was added to precipitate the final product. Previous researchers in our lab tried this method without success. This method was never tried in this study. 
A second synthetic pathway tried by Meyer involved displacement of the carbonate ligand of $\mathrm{Os}(\mathrm{bpy})_{2} \mathrm{CO}_{3}$ by two equivalents of bulky labile solvents followed by displacement of one of the solvent molecules by a less labile molecule. The second ligand would be introduced under much more rigorous conditions than the first. This method, shown by dotted arrows in Figure 4.3, was tried and a $\mathrm{pH}$-independent wave was obtained.

A $50 \mathrm{ml} \mathrm{rbf}$ was filled with $15 \mathrm{ml}$ of dimethylformamide. The solvent was sparged with nitrogen for 10 minutes before $100 \mathrm{mg}$ of $\mathrm{Os}(\mathrm{bpy})_{2} \mathrm{CO}_{3}(0.18 \mathrm{mmol})$ was added. This was followed by addition of 5 drops of 4 -aminomethylpyridine. The mixture was refluxed for one hour and a large excess of water (greater $100 \mathrm{ml}$ ) was added to the hot solution. Saturated ammonium hexafluorophosphate was added to precipitate the final product. The resulting product was filtered, washed with water and dried in a dessicator. The yield was very low, $21 \%$. Because the wave obtained by cyclic voltammetry was independent of $\mathrm{pH}$, it was probably the wrong product. As a result, this synthetic pathway was abandoned.

In the Finklea method, it was observed that although water was a small, less bulky ligand, it could still be displaced by 4-Aminomethylpyridine under harsh conditions. Typically, the method involves addition of about $(250 \mathrm{mg}, 0.44 \mathrm{mmol})$ of $\mathrm{Os}(\mathrm{bpy})_{2} \mathrm{CO}_{3}$ to $50 \mathrm{ml}$ of slightly acidic distilled de-ionized water in $100 \mathrm{ml}$ rbf. 4-Aminomethylpyridine (10 drops) was added and the mixture was refluxed for 4 hours. The solution was then cooled to room temperature and 15 drops of sodium dithionite solution was added before the product was precipitated by addition of ammonium hexafluorophosphate $\left(\mathrm{NH}_{4} \mathrm{PF}_{6}\right)$. Sodium dithionite was added before precipitation to reduce any osmium species that are 
still in higher oxidation states. Yields are usually high, 70-80\%. The open circuit potential for a solution of the product was $30-49 \mathrm{mV}$ negative of the formal potential at all the pHs investigated, indicating that the bulk of the product was in the +2 oxidation state. Figure 4.9 is a UV spectrum for the $\left[\mathrm{Os}(\mathrm{bpy})_{2}(\mathrm{py})\left(\mathrm{OH}_{2}\right)\right]^{2+}$ product. Figure 4.10 and 4.11 show the expected dependence of formal potential on pH. Based on Figure 4.10 and 4.11 and the fact that the product could be attached to the SAM on an electrode, it is believed that the right product was synthesized. Also, the formal potentials and $\mathrm{pK}_{\mathrm{a}} \mathrm{s}$ obtained in Figure 4.11 agree well with those reported by T.J. Meyer and coworkers (Figure 4.1) for a similar compound, $\left[\mathrm{Os}(\mathrm{tpy})(\mathrm{bpy})\left(\mathrm{OH}_{2}\right)\right]^{2+} \cdot{ }^{62}$ The $\mathrm{pK}_{\mathrm{a}} \mathrm{s}$ were calculated by the least squares method. First, Equation 3.2 was used to fit the experimental data. The sum of the squares of the error between the $\mathrm{E}^{0}$ data and values calculated from Equation 3.2 was minimized by the Solver tool in Excel. Three parameters $\mathrm{E}_{2}^{0}, \mathrm{pK}_{\mathrm{a} 1}$ and $\mathrm{pK}_{\mathrm{a} 2}$ were adjusted to give the minimum value for the square of errors. Knowing these three parameters allowed the fourth parameter $\mathrm{E}^{0}{ }_{1}$ to be calculated from equation 3.3. 


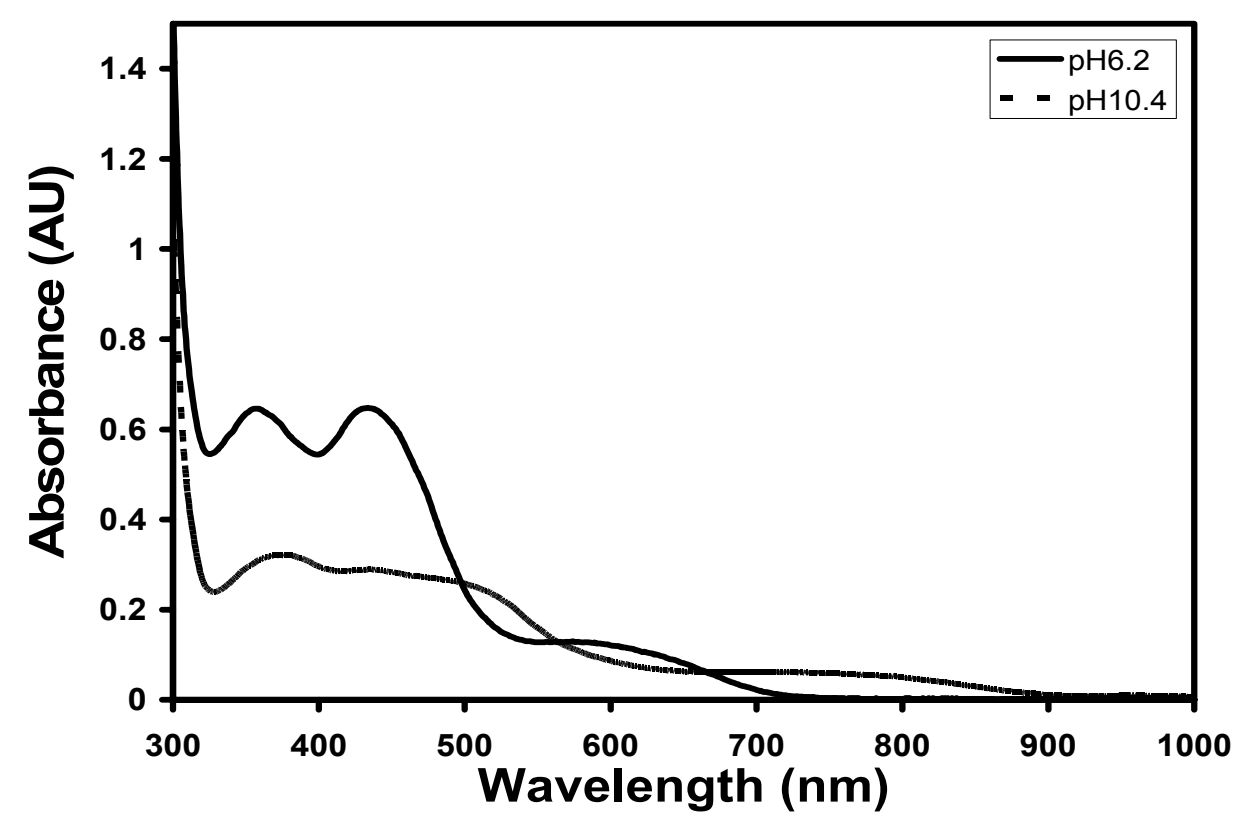

Figure 4.9. UV spectrum for Os(bpy $\left.)_{2}(\mathrm{py})\left(\mathrm{OH}_{2}\right)\right]^{2+}\left(\mathrm{pH} \text { 6.2) and } \mathrm{Os}(\mathrm{bpy})_{2}(\mathrm{py})(\mathrm{OH})\right]^{+}(\mathrm{pH}$ 10.2). Sodium dithionite was added to reduce any residual $\mathrm{Os}^{\mathrm{III}}$ to $\mathrm{Os}^{\mathrm{II}}$.

For the $\mathrm{Os}^{\mathrm{II}}\left(\mathrm{OH}_{2}\right)$ species, peaks were observed at: $357 \mathrm{~nm}\left(\varepsilon \sim 16900 \mathrm{M}^{-1} \mathrm{~cm}^{-1}\right)$; $433 \mathrm{~nm}\left(\varepsilon \sim 16000 \mathrm{M}^{-1} \mathrm{~cm}^{-1}\right)$ and $574\left(\varepsilon \sim 3200 \mathrm{M}^{-1} \mathrm{~cm}^{-1}\right)$, Figure 4.9. The spectrum for $\mathrm{Os}^{\mathrm{II}}(\mathrm{OH})$ (dotted line), shows peaks at: $368 \mathrm{~nm}\left(\varepsilon \sim 8000 \mathrm{M}^{-1} \mathrm{~cm}^{-1}\right)$ and $435 \mathrm{~nm}(\varepsilon \sim 7250$ $\left.\mathrm{M}^{-1} \mathrm{~cm}^{-1}\right)$. There is also a shoulder around $500 \mathrm{~nm}$. The peaks are slightly shifted to lower energies at high $\mathrm{pH}$. The solution color became darker at higher $\mathrm{pH}$ and this color change can be reversed by adding acid. These observations are also consistent with Meyer's observations for the Os(tpy)(bpy) $\left.\left(\mathrm{OH}_{2}\right)\right]^{2+}{ }^{2}$ According to Meyer and coworkers, the shift in wavelength with $\mathrm{pH}$ is caused by the destabilization of the metal $\mathrm{d} \pi$ electrons by $\mathrm{OH}^{-}$relative to $\mathrm{H}_{2} \mathrm{O}$. This causes the electronic transitions from the metal $\mathrm{d} \pi$ orbitals to the antibonding $\left(\pi^{*}\right)$ of the bpy ligands to occur at lower energy. 


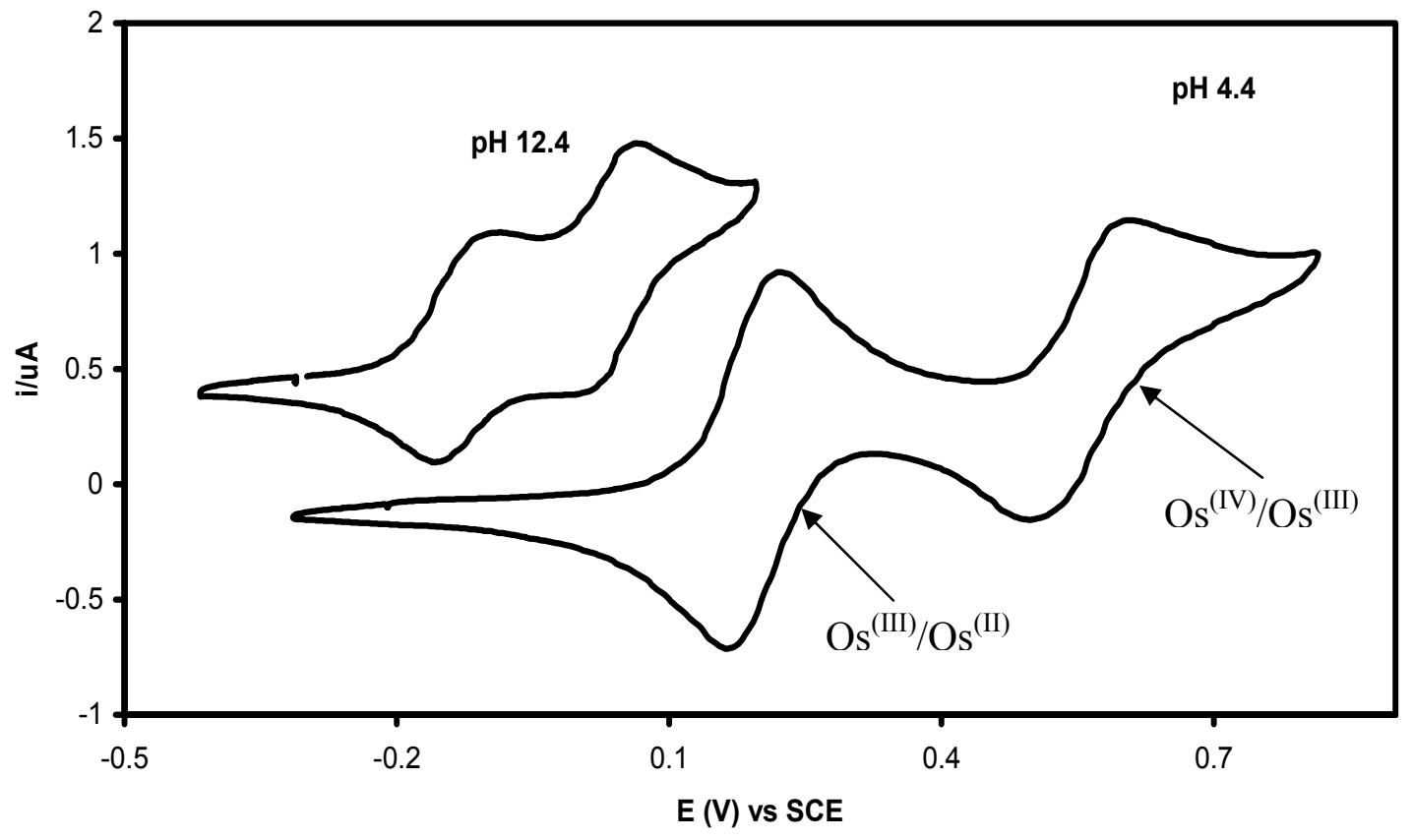

Figure 4.10. CVs for Os(bpy) $\left.)_{2}(\mathrm{py})\left(\mathrm{OH}_{2}\right)\right]^{2+}$ showing both the $\mathrm{Os}{ }^{(\mathrm{III})} / \mathrm{Os}^{(\mathrm{II})}$ wave and the $\mathrm{Os}^{(\mathrm{IV})} / \mathrm{Os}^{(\mathrm{III})}$ wave. Data were collected in $0.5 \mathrm{M} \mathrm{K}_{2} \mathrm{SO}_{4} / 0.1 \mathrm{M}$ Britton-Robinson buffer. Both waves shift to the left as $\mathrm{pH}$ increases. Notice that the $\mathrm{Os}^{(\mathrm{III})} / \mathrm{Os}^{(\mathrm{II})}$ wave merges with the Os ${ }^{(\mathrm{IV})} / \mathrm{Os}^{(\mathrm{III})}$ wave at higher $\mathrm{pH}$ (12.4). The working electrode was a bare gold electrode and the scan rate was $10 \mathrm{mV} / \mathrm{s}$. 


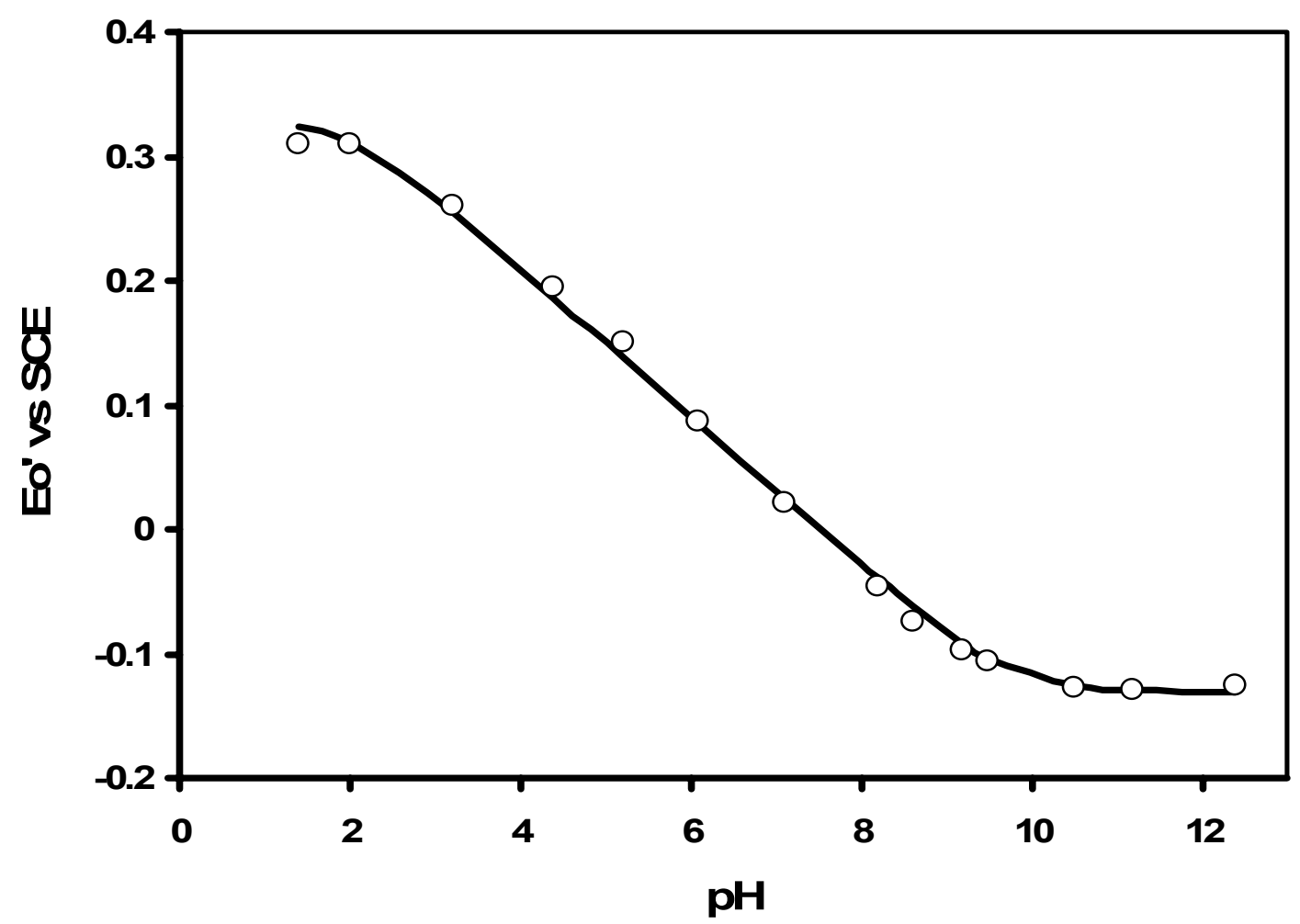

Figure 4.11. Variation of formal potential with $\mathrm{pH}$ of $\left.\mathrm{Os}(\mathrm{bpy})_{2}(\mathrm{py})\left(\mathrm{OH}_{2}\right)\right]^{2+/ 3+}$ in $0.5 \mathrm{M}$ $\mathrm{K}_{2} \mathrm{SO}_{4} / 0.1 \mathrm{M}$ Britton-Robinson buffer, on a bare gold bead electrode, scan rate $=10$ $\mathrm{mV} / \mathrm{s}$. The reference electrode was SCE. The graph has a Nernstian slope of $-59 \mathrm{mV} / \mathrm{pH}$ in the region between $\mathrm{pK}_{\mathrm{a} 1}$ and $\mathrm{pK}_{\mathrm{a} 2} \cdot\left(\mathrm{pK}_{\mathrm{a} 1}=1.96, \mathrm{pK}_{\mathrm{a} 2}=9.73\right)$.

\subsection{Checking Purity}

Three methods were used to confirm the identity and purity of the products. After each synthetic step, a CV and UV-Vis spectrum were often collected. The absence of any extra waves in CV and the UV-Vis spectrum that matched with literature was evidence for the absence of any interfering redox species or impurities within the electrochemical 
or spectroscopic window investigated. In some cases, coulometry was also used to complement these methods.

Coulometry is a convenient method of checking percent purity. In coulometry, one redox species is quantitatively converted to the other and the total charge delivered is measured. For a coulometric study to succeed, the formal potential of the redox reaction must be known and the redox species must be the most easily oxidized or reduced species present, it must not produce side reactions with a different stoichiometry. Moreover, the reactions must proceed with $100 \%$ efficiency. To achieve this, the working electrode must have a large surface area and the solution must be efficiently stirred. Since an opposite reaction occurs at the counter electrode, the working electrode must be isolated from the counter electrode to prevent re-mixing. The choice of the electrolysis potential is based on Nernst Equation:

$$
E=E^{0^{\prime}}-\frac{0.05916}{n} \log \left(\frac{C_{\mathrm{Red}}^{*}}{C_{\text {ox }}^{*}}\right)
$$

at room temperature, where $\mathrm{C}^{*}{ }_{\text {Red }}$ and $\mathrm{C}_{\text {ox }}^{*}$ are the concentration of the reduced and oxidized species respectively, $\mathrm{n}$ is the number of electrons transferred. This Equation predicts how far away from the formal potential the electrolysis potential needs to be set in order to obtain maximum conversion of reactant to product. It can be shown from this Equation that $99 \%$ conversion of the reduced species to the oxidized species occur approximately $120 \mathrm{mV}$ positive of the formal potential.

In a typical coulometric experiment, a three compartment electrode was filled with about $6 \mathrm{ml}$ of a $0.1 \mathrm{M}$ phosphate buffer, $\mathrm{pH}$ 7. A few milligrams of the appropriate osmium complex was added to the cell. The potentiostat was set at a potential at least 120 $\mathrm{mV}$ positive of the formal potential of the $\mathrm{Os}^{\mathrm{II} / \mathrm{III}}$ wave and electrolysis carried out for 
about thirty minutes, after which the solution changed color from a dark brown to a very light brown clear solution. The quantity of charge deposited is recorded and from the Equation:

$n=\frac{Q_{\text {tot }}}{F}$

the number of moles of substance deposited can be calculated. This is compared to the original number of moles added to calculate the percent purity. It is important to note that this process is reversible; if the potential is set at least $120 \mathrm{mV}$ negative of the formal potential, the same charge must be deposited in the reverse process. This was confirmed in this experiment. The solution turned back to the original dark brown color and the same quantity of charge was deposited. A three compartment cell was used to keep the electrodes separate, especially the working and counter electrode. 


\section{CHAPTER 5: GENERAL EXPERIMENTAL PROCEDURES AND INSTRUMENTATION}

\subsection{EXPERIMENTAL.}

5.1.0. Materials. 16-Mercaptohexadecanoic acid, $\mathrm{HS}\left(\mathrm{CH}_{2}\right)_{15} \mathrm{COOH}$ was synthesized in Dr Finklea's lab. 16-Mercapto-1-hexadecanol, $\mathrm{HS}\left(\mathrm{CH}_{2}\right)_{16} \mathrm{OH}$ was purchased from Frontier Scientific and was used without further purification. Absolute ethanol was purchased from AAPER Alcohol and Chemical Company, deuterium oxide (99.9\%) was purchased from Cambridge Isotopes, deuterosulfuric $(97 \% \mathrm{wt})$ acid was purchased from Acros Organics, trideuterophosphoric acid (85\% wt) was purchased from Isotech, potassium deuteroxide, 4-(aminomethyl)pyridine (98\%) and ammonium hexafluorophosphate (99.99\%) were purchased from Aldrich, sodium hydrosulfite, lithium tetraborate (99.9\%), sodium deuteroxide, 1-(3-dimethylaminopropyl)-3ethylcarbodiimide hydrochloride (EDC) were purchased from Fisher Scientific, ammonium hexachloroosmiate(IV) $(99.9 \%$ metals basis, Os $42.5 \%$ ) and gold wires (99.999 \% metals basis) were purchased from Alfa Aesar.

\subsubsection{Preparation of self-assembled monolayers}

As already discussed, self-assembled monolayers are generally prepared by

dissolving a small amount of an appropriate thiol in ethanol. In this study, a small amount of a long chain alkanethiol was dissolved in about $10 \mathrm{ml}$ of ethanol to make a stock solution. This was followed by further dilution of this stock solution to make the monolayer solution of appropriate concentration, typically in the micromolar range. The electrode was then immersed in this solution, upon which the alkanethiol molecules 
spontaneously and rapidly bind to the surface of gold. Typical deposition times are 15 to 24 hours. Details of the experimental procedures are provided below.

5.1.2. Deposition Solution: 16-Mercaptohexadecanoic acid, $\mathrm{HS}\left(\mathrm{CH}_{2}\right){ }_{15} \mathrm{COOH}(5 \mathrm{mg}$, 17.3 micromol) was dissolved in $10 \mathrm{ml}$ of absolute ethanol in a $20 \mathrm{ml}$ scintillation vial. 16-Mercapto-1-hexadecanol, $\mathrm{HS}\left(\mathrm{CH}_{2}\right)_{16} \mathrm{OH}$ (5 mg, 18.2 micromol), was also dissolved in a separate $20 \mathrm{ml}$ scintillation vial containing $10 \mathrm{ml}$ of absolute ethanol. A mixed monolayer deposition solution was prepared by mixing $190 \mu \mathrm{L}$ of the $\mathrm{HS}\left(\mathrm{CH}_{2}\right)_{15} \mathrm{COOH}$ thiol with $10 \mu \mathrm{L}$ of the $\mathrm{HS}\left(\mathrm{CH}_{2}\right)_{16} \mathrm{OH}$ thiol in a third $20 \mathrm{ml}$ scintillation vial containing $2 \%(\mathrm{v} / \mathrm{v})$ of trifluoroacetic acid in $10 \mathrm{ml}$ of absolute ethanol. The final concentration of $\mathrm{HS}\left(\mathrm{CH}_{2}\right)_{15} \mathrm{COOH}$ was $33 \mu \mathrm{M}$ while that of $\mathrm{HS}\left(\mathrm{CH}_{2}\right)_{16} \mathrm{OH}$ was $1.82 \mu \mathrm{M}$. Final coverages of the attached osmium complex on the order of $10^{-11} \mathrm{~mol} / \mathrm{cm}^{2}$ are often obtained with mixed monolayers formed from this solution.

Trifluoroacetic acid preferentially forms hydrogen bonds with free thiol molecules in solution as well as thiol molecules attached to the electrode. When the thiol coated electrode is rinsed with a base, trifluoroacetic acid is washed away leading to formation of a single layer of thiol molecules on the electrode. Shaoyi Jiang et al. observed that thiols prepared in ethanol in the absence of trifluoroacetic acid gave poor quality monolayers due to formation of a double layer by hydrogen bonding. Measurements by X-ray photoelectron spectroscopy (XPS) revealed the presence of unbound thiols on the surface of $-\mathrm{COOH}$ SAMs. ${ }^{29}$ These free thiols are attached to the thiols bound to the electrode through hydrogen bonding. By preparing the thiol solution in a $2 \%(\mathrm{v} / \mathrm{v})$ trifluoroacetic/ethanol solution followed by washing with $10 \%(\mathrm{v} / \mathrm{v})$ 
ammonium hydroxide/ethanol, this layer of unbound thiols was not observed as confirmed by contact angle measurements and XPS.

Electrodes prepared in $100 \% \mathrm{HS}\left(\mathrm{CH}_{2}\right){ }_{15} \mathrm{COOH}$ did not last more than two complete scan rate studies before the redox center was lost. The reason for the loss was probably due to stripping of the monolayer from the electrode. The cause for this behavior is not known. It was observed that, by using mixed monolayers, the electrode survived scan rate studies for the entire $\mathrm{pH}$ range from low $\mathrm{pH}$ to high $\mathrm{pH}$.

\subsubsection{Preparation of Electrodes.}

5.1.3.1. Working electrode: The method reported here is a modification of procedures

reported by other authors. ${ }^{5,25}$ A polycrystalline gold bead electrode was used as a working electrode. The working electrodes were fabricated by cutting a $0.5 \mathrm{~mm}$ gold wire into about $3 \mathrm{~cm}$ length sizes. One end of the wire was melted by meticulously manipulating the wire in a Bunsen burner flame to form the bead. The average bead area was about $0.04 \mathrm{~cm}^{2}$. Two different cleaning procedures were used for the working electrode depending on the application. For bare electrodes, a water rinse followed by flaming to glowing red in a Bunsen burner flame was usually sufficient. Electrodes to which a monolayer would be attached were prepared by chemical etching in piranha solution, which is a 3:1 solution of concentrated sulfuric acid/ hydrogen peroxide (30\%) (CAUTION-piranha solution is hot and can react explosively with organic compounds). The electrodes were rinsed with copious amounts of water followed by electrochemical cycling between $-0.1 \mathrm{~V}$ and $1.4 \mathrm{~V}$ vs $\mathrm{Ag} / \mathrm{AgCl}$ in $0.1 \mathrm{M} \mathrm{H}_{2} \mathrm{SO}_{4}$. A gold oxide layer is formed on the forward scan (oxidation) and stripped off by the reverse scan (reduction). Electrochemical cycling can be repeated until a smooth typical gold oxidation wave is 
obtained with successive scans perfectly superimposed to give a single trace. Chemical etching followed by potential cycling of the gold electrode between -0.1 and $+1.2 \mathrm{~V}$ vs. SCE, has been found to give surfaces that are smooth and reproducible. ${ }^{25}$ After this electrochemical step, the electrodes are rinsed again with lots of water followed by ethanol. The electrodes were blown dry before being immersed in the thiol ethanolic deposition solution overnight. The electrodes were removed and were rinsed with ethanol and then washed twice with $10 \%$ ammonium hydroxide/ethanol solution to wash off any hydrogen bonded trifluoroacetic acid, thereby preventing formation of a second thiol layer. ${ }^{29}$ This was followed by another rinse with $2 \%$ trifluoroacetic acid/ ethanol solution to protonate the carboxylic acid terminal groups of the thiols. Electrodes were placed back in deposition solution for several hours before coupling was done. When electrodes were ready for coupling, the same rinsing procedure was repeated, but, after the ammonium hydroxide step, electrodes were rinsed with water and placed in the coupling solution. This procedure resulted in monolayers of good quality as judged by very low and flat charging currents. This process of rinsing electrodes and placing them back in the deposition solution could be repeated before coupling is done.

5.1.3.2. Counter electrode: A large surface area platinum foil was used as a counter electrode to sustain the large currents produced at the working electrode at high scan rates. The counter electrode was cleaned in two steps. First, it was rinsed with distilled de-ionized water, and, second, it was flamed to a red glow in a Bunsen burner flame. The flaming step was done to remove any residual organic material that could remain after the water rinse step. 
5.1.3.3. Reference electrode: The reference electrode was a commercial 66-E009 premium "no leak" $\mathrm{Ag} / \mathrm{AgCl}$ micro electrode (Cypress). It was stored in saturated potassium chloride solution and rinsed thoroughly with distilled de-ionized water before use.

\subsection{Ascertaining the quality of the Monolayer.}

A good quality monolayer should be able to hold a redox molecule a fixed distance away from the electrode, while at the same time preventing any electrolyte ions from migrating to the electrode surface. A good measure of the quality of the monolayer is based on its blocking behavior, that is, its ability to block these ions. This measure can be easily achieved by calculating the capacitance. Since only the bead of the electrode is immersed in the electrolyte, it is necessary to measure the diameter of the bead in order to calculate its area. This can be easily achieved by using a pair of digital vernier calipers. The capacitance (C) in Faradays, is calculated from the following Equation: ${ }^{30}$

$C=\frac{i_{\text {ch.tot. }}}{2 v}$

where $\mathrm{i}_{\text {ch.tot. }}$ is the total charging current (Amperes) and $v$ is the scan rate in $\mathrm{V} / \mathrm{s}$. Typical capacitance values range from $20-100 \mu \mathrm{F} / \mathrm{cm}^{2}$ for a bare electrode, but can be reduced down to $0.5-10 \mu \mathrm{F} / \mathrm{cm}^{2}$ for a good, tightly packed monolayer coated electrode. When using monolayer coated electrodes, it is important to avoid extremes of potential. At very high potentials, for example, close to $1.4 \mathrm{~V}$, the monolayer can be easily lost due to gold oxidation. At very low potentials, for example, below $-1.0 \mathrm{~V}$, the monolayer can be easily desorbed, especially at high pHs. It is therefore important to know the potential window of the electrode used and to work within these potential limits to avoid damage to the monolayer. At very high scan rates, however, these extreme potentials can be reached 
without deleterious consequences because the time spent at the extreme potentials is too short for any significant faradaic reaction. It is also important to keep the monolayercoated electrode immersed in an aqueous environment, especially after attaching the redox molecule. It was observed that the monolayer with redox molecules attached were not stable when exposed to air. The cause for this instability is not known, but could probably be due to aerial oxidation of the thiol.

\subsection{Coupling Reaction.}

This method was a slight modification of the method used in Dr Finklea's lab previously and involved amide bond formation between the terminal carboxyl group on the thiol and the amine group on the redox center. The major modification from previous procedures was the use of a mixture of acetonitrile and water as a coupling solvent instead of only water. Also, the volume of solvent was cut down from $20 \mathrm{ml}$ to $2 \mathrm{ml}$ as a way of keeping the concentration of the redox molecule high. The monolayer composition was $95 \% \mathrm{HS}\left(\mathrm{CH}_{2}\right)_{15} \mathrm{COOH}$ : $5 \% \mathrm{HS}\left(\mathrm{CH}_{2}\right)_{16} \mathrm{OH}(\mathrm{v} / \mathrm{v})$ instead of a $(50 / 50)$ mixture used by Haddox. This composition resulted in both good quality monolayers and good coverage of the redox molecule.

Several different coupling methods were tried without success. Two major problems were encountered. First, coupling in aqueous solutions resulted in voltammograms with slow transient changes of the charging current when the sweep potential was reversed. The cause for this behavior is not known. The second problem was the presence of an impurity wave. This impurity wave was observed especially on the cathodic side of the voltammogram and appeared to be independent of $\mathrm{pH}$. Also some 
undissolved residual material always remained when the redox molecule was dissolved in water.

The impurity wave could be $\left[\mathrm{Os}(\mathrm{bpy})_{2}(\mathrm{py})_{2}\right](\mathrm{PF} 6)_{2}$ which is also a possible product in the reaction mixture. The assumption is that 4-Aminomethylpyridine displaces a single water ligand in the bis-aquo complex (stage III) of synthesis. However, it is also possible that both aquo ligands are displaced by 4-Aminomethylpyridine. While there was no evidence of the bis (py) product in the synthesized osmium complex, a trace of this compound could still undergo the amide coupling reaction preferentially. Also, since this product has no aquo ligand, its electrochemical properties will be independent of $\mathrm{pH}$. However, attempts to purify the $\left[\mathrm{Os}(\mathrm{bpy})_{2}(\mathrm{py})\left(\mathrm{OH}_{2}\right)\right]^{2+}$ by column chromatography using neutral alumina were unsuccessful, mostly due to the fact that the amount of sample was too little and most of it was trapped in the column. Three bands were eluted, but the amount of sample isolated from each band was too small for any meaningful analysis.

In Haddox's method, coupling was done in phosphate buffer at neutral $\mathrm{pH}$. Besides the coupling method reported by Haddox, ${ }^{45}$ another method tried was performing the coupling reactions in two steps. Amide bond formation in the presence of EDC occurs in two important steps ${ }^{69,70}$ The first step involves reaction of EDC with the - $\mathrm{COOH}$ group to form an O-acylurea derivative of EDC as an intermediate. This intermediate is unstable, and, if an amine group is present, it quickly reacts to form the amide bond. However due to the instability of the intermediate, it can also hydrolyse in aqueous solutions or rearrange to an $\mathrm{N}$-acylurea adduct, resulting in poor yields of the final product. N-hydroxysulfosuccinimide, (NHSS), is often added along with EDC to form the relatively stable succinimide ester. This ester is readily displaced by an amine to form 
an amide. ${ }^{69}$ Phosphate is also believed to react with EDC causing it to lose its efficacy as a coupling agent. ${ }^{69,70}$

EDC works best as a coupling agent at $\mathrm{pH}$ between 4 and 5 in the absence of phosphate buffer. However, the amide bond formation occurs optimally at higher pHs, above $\mathrm{pK}_{\mathrm{a}} \mathrm{s}$ of the amine group where it is not protonated. The challenge was to combine these reactions in order to activate the $-\mathrm{COOH}$ group at low $\mathrm{pHs}$ where the reaction with $\mathrm{EDC}$ is fast and efficient, at the same time forming the amide bond at higher $\mathrm{pH}$ where the amine group is deprotonated. To achieve this goal, the two reactions were separated. The $-\mathrm{COOH}$ group was activated at pH 5 using $0.1 \mathrm{M}$ (2[N-morpholino]ethanesulfonic acid) (MES) in the presence of NHSS for about 10 minutes. The electrodes were then rinsed thoroughly with water before placing them in the coupling solution containing the redox molecule in $0.1 \mathrm{M}$ phosphate buffer at $\mathrm{pH}$ 9. This reaction worked well, but the problems encountered before did not go away. The impurity wave was still present and the CVs still showed slow transient change when the potential was reversed.

In acetonitrile, however, the coupling reaction works much better even in the absence of NHSS. The redox molecule dissolved readily in acetonitrile leaving no residual insoluble material, although this could also mean that the impurity dissolved. Although the redox molecule dissolved readily in acetonitrile, EDC was only sparingly soluble in that solvent. This resulted in poor coverage of the redox molecule on the electrode. To go round this problem, it was noted that, when a few drops of water were added to acetonitrile, EDC dissolved easily and the coverage of the redox molecule on the electrode increased dramatically. Other coupling solvents tried include ethanol acetone and dimethylformamide. Despite the fact that good coverage was also obtained in 
ethanol and DMF, the voltammograms showed the same slow transient changes observed in water when the scan direction was reversed. Acetonitrile was found to give the best results, in terms of coverage and shapes of voltammograms. Because of this, all coupling reactions were done in acetonitrile/ water mixture. Approximately 5 drops of water in 2 $\mathrm{ml}$ of acetonitrile was found to be an optimum balance between the aqueous and nonaqueous nature of the coupling solution.

The redox molecule $\left[\mathrm{Os}(\mathrm{bpy})_{2}(\mathrm{py})\left(\mathrm{OH}_{2}\right)\right]^{2+}$ was coupled to carboxylic acid terminal groups in a mixed $\mathrm{HS}\left(\mathrm{CH}_{2}\right)_{15} \mathrm{COOH} / \mathrm{HS}\left(\mathrm{CH}_{2}\right)_{16} \mathrm{OH}(95: 5(\mathrm{v} / \mathrm{v}) \%)$ SAM, using the water-soluble carbodiimide, (EDC). About 2-3 mg of the osmium aquo complex was dissolved in approximately $2 \mathrm{ml}$ of acetonitrile to which 5 drops of water had been added. About $10 \mathrm{mg}$ of EDC was added to the coupling solution. Electrodes were removed from the thiol deposition solution and rinsed twice with $10 \% \mathrm{NH}_{4} \mathrm{OH} /$ ethanol solution, followed by water. The electrodes were then placed in the coupling solution for 35 minutes, after which they were rinsed copiously with water before use. Electrodes were stored in water until they were ready for use. Exposure of the electrode to air resulted in the monolayer being lost faster, possibly due to oxidation of the thiol by oxygen.

\subsection{Thermodynamic information of Redox molecules attached to SAMs.}

Generally, two methods are used to attach a redox molecule to the electrode via a SAM. The first method involves depositing the monolayer on the electrode followed by attachment of the redox molecule as described above. The second method requires a redox molecule to be attached to the thiol first; the thiol with the redox molecule attached is then attached to the electrode. In each case, a diluent SAM can be used with the electroactive SAM to control the coverage. The purpose of the diluent SAM is to keep the 
redox molecules as far apart as possible to minimize double layer effects and electron transfer between adjacent osmium complexes on the SAM. More details about this will be provided in sections to follow.

Ideally, for a reversible system, the cyclic voltammogram (CV) of the redox molecule at very low scan rates should be symmetrical, with a peak splitting of zero. Under these conditions, the system is said to be under thermodynamic control(Figure 5.1)

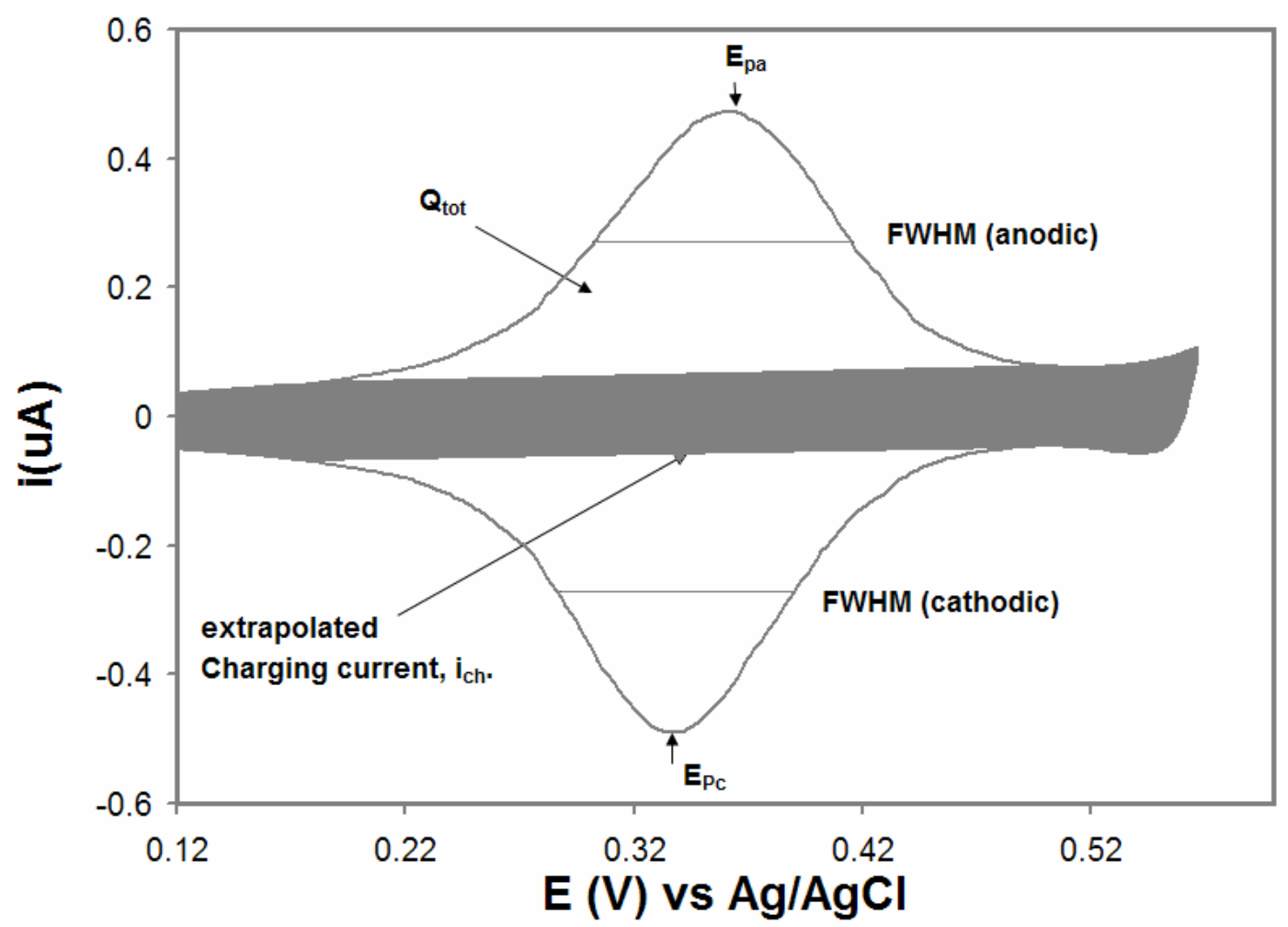

Figure 5.1. Reversible CV showing faradaic and non-faradaic (charging) current. The area under each peak gives the total charge deposited on each scan.

However, for practical purposes, a peak splitting less that $100 \mathrm{mV}$ is considered to be reversible. The area under each peak gives the total charge, $\mathrm{Q}_{\text {tot }}$ (Figure 5.1) and 
should be equal for both anodic and cathodic reactions. The area under each peak can be calculated by integration:

$Q_{\text {tot }}=\int i_{f} d t$

Knowing the total charge, the coverage of the redox molecule can be calculated from:

$\Gamma=\frac{Q_{\text {tot }}}{n F A}$

where $\mathrm{n}$ is number of electrons transferred, $\mathrm{F}$ is the Faradaic constant, and $\mathrm{A}$ is the area of the bead. The area of the electrode can be calculated by carefully measuring the diameter of the bead using a digital vernier calipers and then calculating the area from the Equation: $\mathrm{A}=4 \pi \mathrm{r}^{2}$. Another way of calculating the area of the electrode is collecting a $\mathrm{CV}$ of a redox molecule freely diffusing in solution, on a bare electrode. The diffusion limited peak current is related to the electrode area by the Equation:

$i_{p}=\left(2.69 \times 10^{5}\right) n^{\frac{3}{2}} A D_{0}^{\frac{3}{2}} V^{\frac{1}{2}} C_{0}^{*}$

$\mathrm{n}$ is the number of electrons transferred, $\mathrm{D}_{0}$ is the diffusion coefficient, typically $10^{-6}$ $\mathrm{cm}^{2} / \mathrm{s}$ in aqueous solutions, $v$ is the scan rate and $\mathrm{C}_{0}{ }^{*}$ is the concentration of the redox species in the bulk solution in $\mathrm{mol} / \mathrm{cm}^{3}$.

The redox species used for this purpose was a ruthenium complex, $\mathrm{Ru}\left(\mathrm{NH}_{3}\right)_{3} \mathrm{Cl}_{3}$. This compound has well known and well behaved electrochemical properties. By collecting a $\mathrm{CV}$ under reversible conditions, the peak current can be measured and used to calculate the area of the electrode. In this study, the electrode area was calculated using both techniques and there was very good agreement between the two values obtained. A value of $0.02 \mathrm{~cm}^{2}$ was obtained using both techniques. 
Figure 5.1. above is a typical voltammogram of an attached redox system under reversible conditions. The peak splitting is obtained from the difference between the peak potentials $\left(\mathrm{E}_{\mathrm{pa}}-\mathrm{E}_{\mathrm{pc}}\right)$. The average of the peak potentials gives the formal potential. FWHM is the peak at half peak height. For an ideally behaved system, FWHM should be equal to $\frac{3.5 R T}{n F}$ or $\frac{91 m V}{n}$ at room temperature. ${ }^{72}$ A broader peak, which manifests itself as $\mathrm{E}_{\mathrm{FWHM}}$ higher than $91 \mathrm{mV} / \mathrm{n}$, is often a sign of the presence of multiple thermodynamic environments (thermodynamic heterogeneity). Several factors could be responsible for this phenomenon. Due to imperfections in the monolayer (surface heterogeneity), some redox molecules can be partially buried in the monolayer. Consequently, these redox molecules will interact differently with the solvent compared to the rest of the redox molecules, leading to a spread of formal potentials. Also, changes in the local electrostatic potential during oxidation or reduction of the redox molecule could result in peak broadening due to double layer effects ${ }^{35}$. However, double layer effects can be minimized by keeping the coverage of the redox molecule low. This is often achieved by using a diluent thiol, a thiol with no redox molecule attached. The assumption is that, by mixing an electroactive thiol with a diluent thiol, the electroactive thiols will pack themselves in such a way that they are as far away as possible to minimize lateral interactions $^{36}$. The space between them is then filled by the diluent thiols and the equilibrium concentration depends on the relative compositions of the two thiols. Annealing the electrode by placing it in a pure diluent SAM has also been reported to reduce coverage $\mathrm{e}^{35}$. This is because the diluent monolayer chains exchange with the electroactive monolayer thiols when the two are mixed together. The effects of thermodynamic heterogeneity was kept to a minimum in this study by careful control of 
the deposition conditions such as ratio of electroactive thiol to diluent thiol as well as controlling the coupling conditions. Annealing was not used in this study, because it resulted in a decrease in the coverage of the redox molecule without a concomitant decrease in the charging current. Other important factors to consider include concentration of redox molecule, coupling time, concentration of coupling agent, EDC, coupling solvent and temperature. For the osmium system, there seemed to be a complex and delicate balance between the concentration of redox center, the coupling time and the concentration of EDC.

\subsection{Electrochemical studies.}

All data were collected using cyclic voltammetry on a potentiostat/galvanostat (Princeton Applied Research, PAR (Model 283)) connected to a digital oscilloscope (Tektronix TDS430A) and a waveform Generator (PAR Model). The oscilloscope was in turn connected to a computer via a GPIB interface for easy data transfer and manipulation. Data were transferred from the oscilloscope to the computer using Wavestar software (National Instruments) and then saved as CSV file.

A three electrode system was used in a single compartment miniature cell (Princeton Applied Research polarographic cell). This cell design was used in order to minimize the effect of uncompensated resistance by keeping the distance between the reference electrode and working electrode small. The product of current and uncompensated resistance is termed $\mathrm{iR}$ drop and values less than $10 \mathrm{mV}$ are usually considered insignificant. Besides decreasing the distance between the working electrode and the reference electrode, $\mathrm{iR}$ drop can also be reduced by increasing the concentration of electrolyte, since it is inversely proportional to resistance. The cell design was also a 
useful and conservative design for experiments done in deuterated electrolytes. Because of the high cost of deuterium oxide and deuterated salts, small volumes $(\sim 4 \mathrm{ml})$ of electrolyte were used. Buffers of different $\mathrm{pHs}$ were prepared by pre-adjusting the $\mathrm{pH}$ of the stock buffer to the appropriate $\mathrm{pH}$ using concentrated solutions of either acid or base. About $4 \mathrm{ml}$ of buffer solution was used in each case.

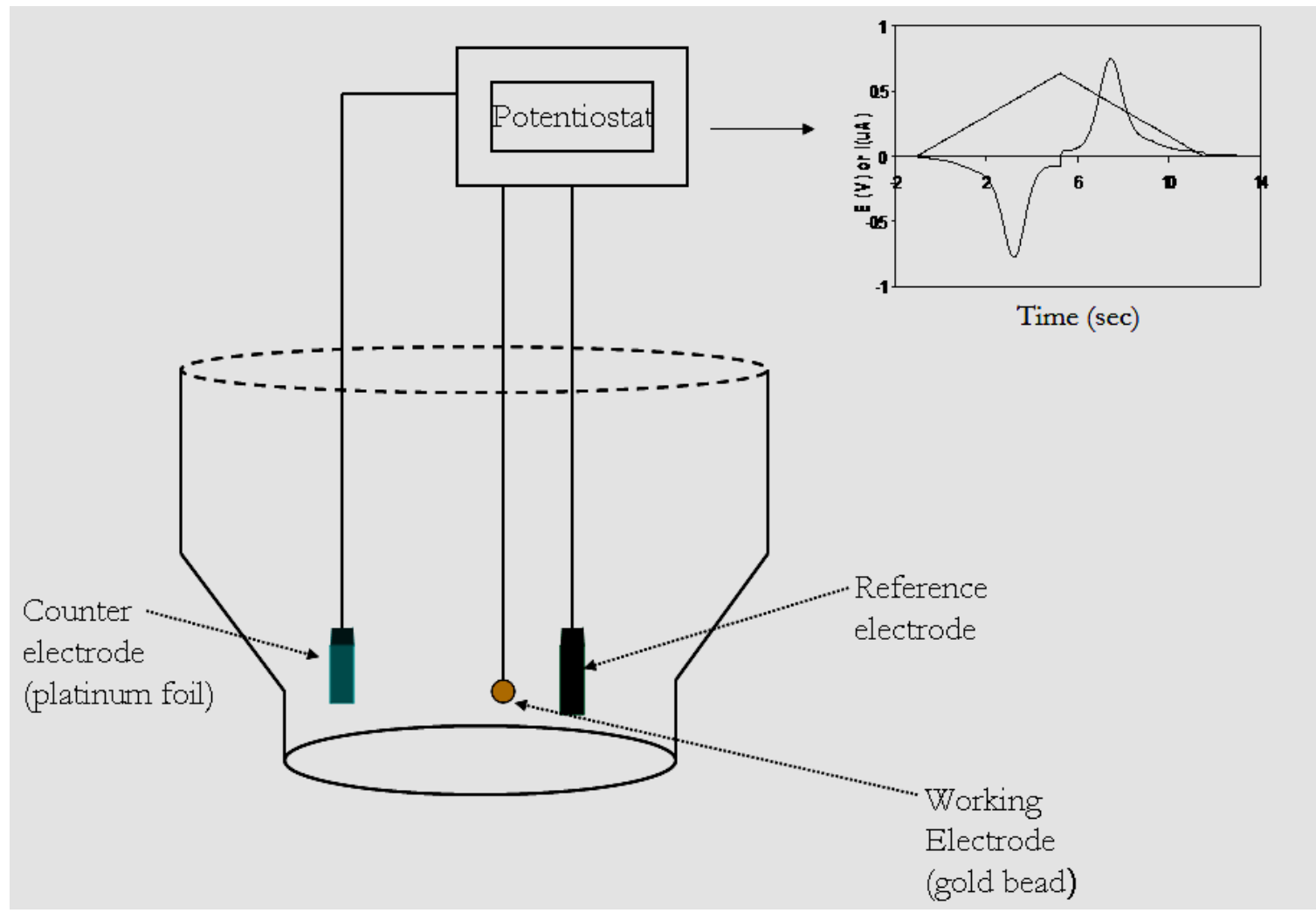

Figure 5.2. Generalized diagram of experimental set up

A previous cell design had three separate compartments and the three electrodes were isolated from each other by porous frits. This cell design used an electrolyte volume of up to $30 \mathrm{ml}$. Because of the size of the cell, it was easier and faster to adjust the $\mathrm{pH}$ by adding acid or base to the central compartment and monitoring the $\mathrm{pH}$ using the standard glass $\mathrm{pH}$ electrode. However, the distance between the working electrode and the 
reference electrode was large which could also lead to higher uncompensated resistances. The other problem stemmed from temperature fluctuations. Due to the exothermic nature of the reactions, addition of concentrated acid or base to aqueous solutions results in an increase in temperature. Since $\mathrm{pH}$ measurements are temperature-dependent, this led to $\mathrm{pH}$ fluctuations within the time window of the experiment. By pre-adjusting the $\mathrm{pH}$ of the buffers, the $\mathrm{pH}$ could be measured after the buffer had cooled down to room temperature, thereby obtaining an accurate reading. Although this procedure could also be done with the large three compartment cell, this would require even larger volumes of buffer solution. Because of the large sample volumes used and the uncertainties in the estimation of uncompensated resistance on this cell, the single compartment cell design was adopted for all measurements.

All electrolyte solutions were prepared using distilled de-ionized water that had been passed through a Barnstead Mega-Pure water purifier/deionizer. The resistivity of this water was $16 \mathrm{M} \Omega-\mathrm{cm}$. The electrolyte was prepared starting with 1.0 M Sulfuric Acid and $0.1 \mathrm{M}$ each of sodium phosphate, sodium citrate and sodium borate, to ensure that the electrolyte is buffered in approximately the whole $\mathrm{pH}$ range (1-11) investigated. Electrolyte $\mathrm{pH}$ was measured using a standard glass $\mathrm{pH}$ electrode (Fisher Scientific). About $4 \mathrm{ml}$ of the stock buffer solution was added to a $20 \mathrm{ml}$ scintillation vial and the $\mathrm{pH}$ was adjusted using a few drops of either concentrated sulfuric acid or potassium hydroxide prior to use. Concentrated solutions were used to (a) minimize uncompensated resistance in the cell, (b) minimize double layer effects, and (c) avoid significant changes in the ionic strength of the electrolyte during $\mathrm{pH}$ adjustments. Potassium hydroxide was used in preference to sodium hydroxide in order to minimize sodium error at high $\mathrm{pHs}$. 
The buffers were sparged using either nitrogen or argon just before use to remove dissolved oxygen. Dissolved oxygen is often reduced within the potential window of most electrochemical reactions, hence the need to remove it prior to analysis. Data were collected between $\mathrm{pH} 1$ and 11.

At each $\mathrm{pH}, \mathrm{CV}$ data were collected at scan rates of $0.1,1,10,100$ and $1000 \mathrm{~V} / \mathrm{s}$. The initial potential was set at a value negative of the $\mathrm{Os}^{\mathrm{IIIIII}}$ formal potential, scanned to a value sufficiently positive to fully oxidize all of the osmium complex to Os ${ }^{\mathrm{III}}$, scanned to a value sufficiently negative to fully reduce all of the osmium complex to Os ${ }^{\mathrm{II}}$ and then returned to the initial potential. For faster scan rates of 100 and $1000 \mathrm{~V} / \mathrm{s}$, the switching potentials were usually beyond the potential associated with gold oxidation or monolayer desorption, but rapid potential excursions at these scan rates did not result in any significant degradation of the monolayer. 


\subsection{Kinetic Isotope effect}

In order to investigate the effect of the deuteron on the rate of PCET reactions, experiments were repeated in deuterated buffers and deuterated salts. $\mathrm{pH}$ measurements were carried out using an Oakton $\mathrm{pH}$ meter and a standard glass electrode. The electrode was calibrated using standard aqueous buffers. $\mathrm{pH}$ measurements in heavy water requires a correction factor if the electrode is calibrated using buffers prepared in light water. ${ }^{73-76}$ The $\mathrm{pH}$ electrode response is based on an equilibrium between ions in solution and groups on the surface of the membrane of the electrode. This equilibrium occurs at the interface between the solution and the surface of the membrane, creating a potential that is dependent on the activity of the ions in the contacting solution. Although the mechanism of response has been reported to be the same in both light water and heavy water, it is the difference in the two isotopes, also known as the "isotope effect", that is key. In water, the equilibrium is between the proton and the surface groups while in deuterium oxide the equilibrium is now set up between the surface groups and the deuteron. Some work has been done to find a correlation between $\mathrm{pH}$ measurements done in $\mathrm{D}_{2} \mathrm{O}$ and those done in $\mathrm{H}_{2} \mathrm{O} \cdot{ }^{73-76}$ Earlier reports suggested applying a correction factor of 0.4 added to the $\mathrm{pH}$ reading obtained in $\mathrm{D}_{2} \mathrm{O}$ to convert it to $\mathrm{pD}$, that is $\mathrm{pD}=\mathrm{pH}^{*}+$ $0.41{ }^{73-75}$ However, recent experimental studies have shown that a more accurate correction is obtained using the modified Equation. ${ }^{76}$

$\mathrm{pD}=0.929 \mathrm{pH}^{*}+0.42$

where $\mathrm{pD}$ is the $\mathrm{pH}$ corrected for the deuteron, and $\mathrm{pH}^{*}$ is the apparent $\mathrm{pH}$ reading from the $\mathrm{pH}$ meter in deuterium oxide. 
The electrodes were prepared and coupled as described above. The electrolyte was prepared starting with $1.0 \mathrm{M} \mathrm{D}_{2} \mathrm{SO}_{4}$ in $0.1 \mathrm{M}$ Britton-Robinson buffer. The buffer was prepared from anhydrous lithium tetraborate, $\mathrm{D}_{3} \mathrm{PO}_{4}$ and anhydrous tri-sodium citrate. Anhydrous tri-sodium citrate was prepared by evaporating two moles of water of crystallization from the hydrated salt in an oven. The $\mathrm{pH}$ was adjusted using deuterosulfuric acid $\left(\mathrm{D}_{2} \mathrm{SO}_{4}\right)$ and potassium deuteroxide (KOD).

\subsection{Cyclic Voltammetry}

Cyclic voltammetry is one of the most versatile electroanalytical techniques, which can also be used for qualitative diagnosis of electrode reactions. It has the ability to generate a species in one scan and probe its fate with subsequent scans. Additionally, cyclic voltammetry provides a uniquely convenient way of separating charging current from faradaic current. Because of these attributes, cyclic voltammetry provides a rapid and convenient way of obtaining both thermodynamic and kinetic information of a redox system. The waveform generator used in this study is capable of reaching scan rates up to $1000 \mathrm{~V} / \mathrm{s}$ which is necessary to obtain kinetic information. Thermodynamic properties such as the formal potential $\mathrm{E}^{\mathrm{o}}$, are obtained at the lowest scan rates:

$$
E^{o}=\frac{E_{p}^{a}+E_{p}^{c}}{2}
$$

where $E_{p}^{a}$ is the anodic peak potential and $E_{p}^{c}$ is the cathodic peak potential.

\subsection{DATA ANALYSIS.}

The data were analyzed on a Microsoft Excel spreadsheet to obtain the reversible formal potential, the standard rate constants, Tafel plots and transfer coefficients vs 
overpotential. The data were corrected for charging current at each $\mathrm{pH}$. The uncompensated resistance was measured by chronoamperometric techniques and iR drop corrections were only applied at high scan rates $(100 \mathrm{~V} / \mathrm{s}$ and $1000 \mathrm{~V} / \mathrm{s})$ at each $\mathrm{pH} . \mathrm{R}_{\mathrm{u}}$ values obtained ranged from 10 to $20 \mathrm{ohms}$.

\subsubsection{Obtaining Kinetic information.}

In this section, a detailed procedure for obtaining kinetic information is provided. Because of the large volume of calculations involved in this study, it was imperative to develop a program for data treatment in order to expedite data analysis. Microsoft Excel proved to be a powerful program for both graphing and numerical data analysis. Typically, five CVs are collected at $0.1,1,10,100$ and $1000 \mathrm{~V} / \mathrm{s}$ at each $\mathrm{pH}$. The lowest scan rate is assumed to correspond to reversible behavior (thermodynamic control) and is used to calculate the formal potential using Equation 5.6. The difference between the applied potential (E) and the formal potential $\left(\mathrm{E}^{0^{\prime}}\right)$ is the overpotential $(\eta)$ and is the driving force for electron transfer.

Under equilibrium conditions, the rate of electron transfer is faster than the rate at which the potential is scanned. Under such conditions, no kinetic information can be obtained. For useful kinetic information to be obtained, the peak splitting should be at least $120 \mathrm{mV}$, which typically occurs at scan rates of 10,100 and $1000 \mathrm{~V} / \mathrm{s}$.

The formal potential calculated for the lowest scan rate $(0.1 \mathrm{~V} / \mathrm{s})$ is used for data analysis at all the other higher scan rates. The non-faradaic current is subtracted for the CVs at higher scan rates $(10,100$ and $1000 \mathrm{~V} / \mathrm{s})$ by extrapolating the charging current before and after the peak. The total charge is calculated by integrating the area under the 
anodic wave and cathode wave for each scan rate, Equation 5.01. The kinetically controlled faradaic current $i_{f}$ is given by:

$i_{f a}=k Q_{\mathrm{Red}}$

(5.7) for oxidation

and

$i_{f c}=k Q_{o x}$

(5.8) for reduction

$Q_{\text {Red }}$ is the total charge for the reduced species $Q_{o x}$ is the total charge for the oxidized species. The respective rate constants can be obtained from Equations 5.7 and 5.8 as:

$k_{a}=\frac{i_{f a}}{Q_{\text {Red }}}$

and

$k_{c}=\frac{i_{f c}}{Q_{o x}}$

A challenge in applying these Equations is the fact that the rate constants have been observed to vary depending on which point on the voltammogram the rate constant is calculated, a condition often known as kinetic heterogeneity (spread of rate constants on a SAM at a given overpotential) ${ }^{15,72,77,78}$. Ideally, a single rate constant should be observed at a given overpotential irrespective of the scan rate, especially at driving forces sufficiently far away from the equilibrium position or formal potential. In practice, for any current transient, rate constants at given overpotentials tend to be higher when the measurement is performed at shorter times (smaller percentage of redox centers converted to product) than at longer times (larger percent of redox species converted to product). Because of this problem, rate constants were calculated at potentials corresponding to $50 \%$ conversion ( $50 \%$ of redox couple oxidized or reduced to the final oxidation state). The overpotential at $50 \%$ conversion is corrected for iR drop using the following Equation: 
$\eta_{0.5}=E_{0.5}-E^{0 ;}-i_{0.5} R_{u}$

$R_{u}$ is the uncompensated resistance, $E$ is the applied potential. The subscript 0.5 denotes the point of $50 \%$ conversion. This point is obtained from the spreadsheet which is set up to calculate the fraction of reactant molecules remaining. The corresponding data for current, overpotential and applied potential are read at $\mathrm{Q}_{\mathrm{f}}=0.5$. Note that the current used here is the total current before subtracting charging current. This strategy produces a consistent average rate constant and greater precision in the data at all potentials. The anodic rate constant is then given by:

$k_{a}=\frac{i_{f a}}{Q_{\text {tot }}\left(Q_{0.5}-Q_{\text {Nernst }}\right)}$

and the cathodic rate constant is given by:

$k_{c}=\frac{i_{f c}}{Q_{\text {tot }}\left(Q_{0.5}-Q_{\text {Nernst }}\right)}$

$\mathrm{Q}_{\text {Nernst }}$ is the theoretical fractional charge remaining at the overpotential, as defined by the Nernst Equation, or the charge which should remain if the redox molecules were in equilibrium with the instantaneous overpotential. If the overpotential is greater than 120 $\mathrm{mV}$, then $\mathrm{Q}_{\text {Nernst }}$ is essentially zero, and Equations (5.12) and (5.13) collapse to Equations (5.9) and (5.10).

The rate constants are plotted against the overpotential obtained from Equation 5.10 to yield Tafel plots $(\ln (\mathrm{k})$ vs $\eta)$. Each scan rate yield two data points on the Tafel plot, one in the anodic branch (positive $\eta$ ) and one in the cathodic branch (negative $\eta$ ). As the scan rate is increased, the point corresponding to $\eta_{0.5}$ occurs at larger and larger overpotentials. At large overpotentials, Tafel plots begin to show curvature, and, provided that this curvature is significantly large, the reorganization energy can be 
estimated by fitting Tafel plots to theoretical plots of various reorganization energies (working curves).

A more convenient way to obtain the reorganization energy involves plotting the transfer coefficient against overpotential. If both the oxidized and reduced species have the same reorganization energy, this plot should be linear and both the cathodic branch and anodic branch can be fitted by a single straight line. The slope of the line is related to the reorganization energy by Equation 1.36 (Chapter 1). However, if the two redox species have different reorganization energies, the plot of $\alpha$ vs $\eta$ exhibits different slopes for the oxidized and reduced species. In such cases, it is more convenient to fit the two lines separately as shall be seen in Chapter 6 . The slopes are then calculated separately for each branch yielding two different $\lambda$ values. Different reorganization energies between the reduced and oxidized species result in asymmetric Tafel plots. 


\section{CHAPTER 6: PROTON-COUPLED ELECTRON TRANSFER, KINETIC STUDIES OF POLYPYRIDYL OSMIUM COMPLEXES}

\subsection{Results}

The redox molecule, $\left[\mathrm{Os}(\mathrm{bpy})_{2}(\mathrm{py})\left(\mathrm{OH}_{2}\right)\right]^{2+}$ was attached to an electrode via a (90/10) $\mathrm{HS}\left(\mathrm{CH}_{2}\right){ }_{15} \mathrm{COOH} / \mathrm{HS}\left(\mathrm{CH}_{2}\right)_{15} \mathrm{CH}_{2} \mathrm{OH}$ self-assembled monolayer. All coupling reactions were carried out in acetonitrile at low EDC concentration and all data analyses was based on the first wave, Os ${ }^{\text {III/II }}$ of the osmium complex, (Figure 4.9), because the second wave, Os ${ }^{\mathrm{IV} / \mathrm{III}}$ overlapped with gold oxidation. Figure 6.1 shows a typical CV under reversible conditions for the attached redox molecule. Typical peak splittings were less than $100 \mathrm{mV}$ and peak half widths were approximately $100 \mathrm{mV}$ at all pHs, showing nearly ideal behavior. At $25{ }^{\circ} \mathrm{C}$, ideally behaved CVs have a typical peak half width of 91 $\mathrm{mV}$ for a single electron transfer process. The ratio of peak areas was always close to unity at the lowest scan rate showing that the system was reversible.

Figure 6.2 (a) shows the $\mathrm{pH}$ dependence of the formal potential for the $\left[\mathrm{Os}(\mathrm{bpy})_{2}(\mathrm{py})\left(\mathrm{OH}_{2}\right)\right]^{2+}$ complex in light water. Figure $6.2(\mathrm{~b})$ is a corresponding plot in heavy water (deuterium oxide). The solid line is a theoretical fit of the data based on Equation 3.2. Thermodynamically, the experimental results agree with predicted behavior of the square scheme for a 1e,1H system. The theoretical line fits the data very well. 


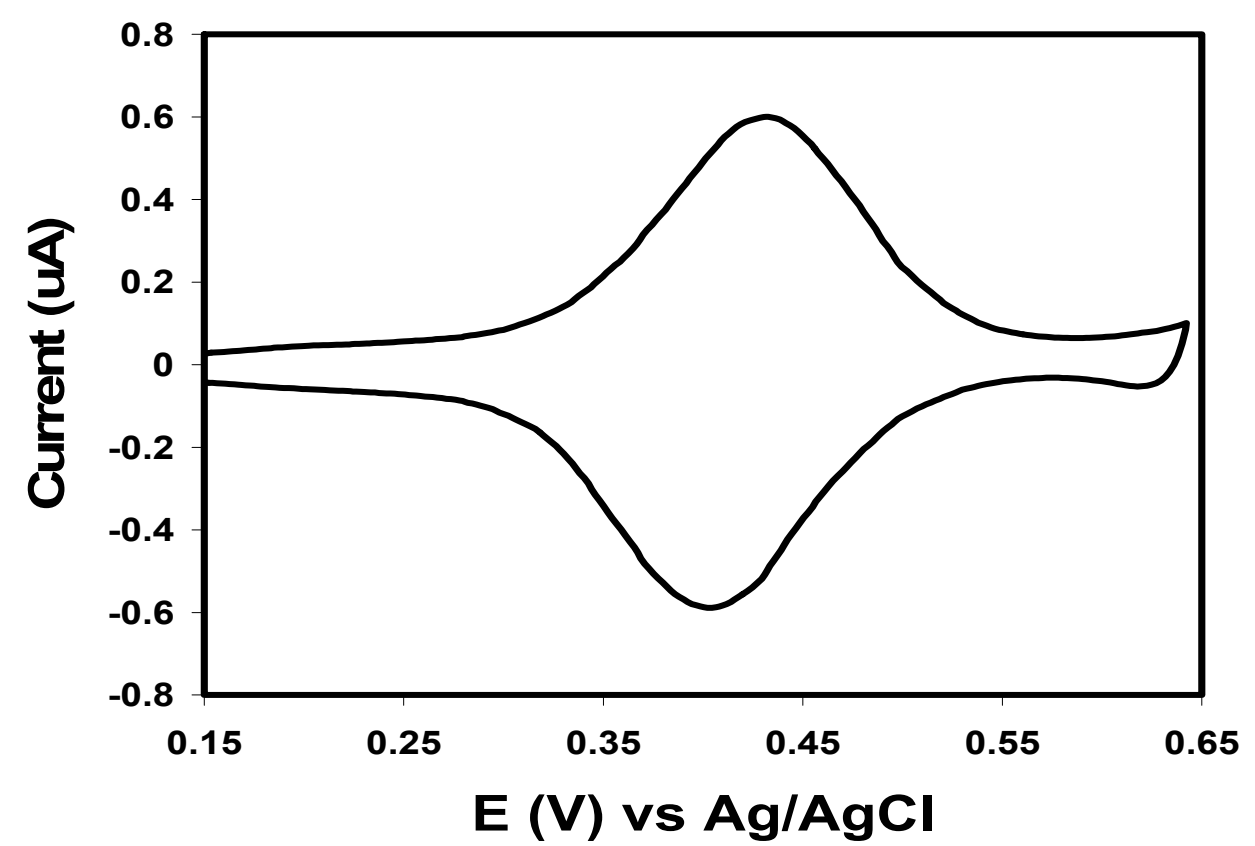

Figure 6.1. Typical CV at $0.1 \mathrm{~V} / \mathrm{s}$ for $\left[\mathrm{Os}(\mathrm{bpy})_{2}(\mathrm{py})\left(\mathrm{OH}_{2}\right)\right]^{2+}$ attached to a $(90 / 10)$ $\mathrm{HS}\left(\mathrm{CH}_{2}\right)_{15} \mathrm{COOH} / \mathrm{HS}\left(\mathrm{CH}_{2}\right)_{15} \mathrm{CH}_{2} \mathrm{OH}$ self-assembled monolayer on an electrode. The electrolyte was prepared starting with $1.0 \mathrm{M} \mathrm{H}_{2} \mathrm{SO}_{4}$ in $0.1 \mathrm{M}$ Britton-Robinson buffer and adjusting the $\mathrm{pH}$ to 1.5 , using $\mathrm{KOH}$.

As shown in Figures 6.2 (a) and (b), three distinct regions can be observed for the two graphs. At the $\mathrm{pH}$ extremes, the graphs level off and the formal potential becomes independent of $\mathrm{pH}$. At intermediate $\mathrm{pHs},\left(\mathrm{pK}_{\mathrm{a} 1}<\mathrm{pH}<\mathrm{pK}_{\mathrm{a} 2}\right)$, the formal potential shows a linear variation with $\mathrm{pH}$, with a Nernstian slope of $-60 \mathrm{mV} / \mathrm{pH}$. The cause of the behavior at extreme $\mathrm{pH}$ is related to the onset of pure electron transfer processes. At $\mathrm{pH}$ lower than $\mathrm{pK}_{\mathrm{a} 1}$, the dominant redox species are the fully protonated species (bottom of scheme I, see Figure 3.1 in Chapter 3) which undergo pure electron transfer. Similarly, at $\mathrm{pH}$ greater than $\mathrm{pK}_{\mathrm{a} 2}$, the redox species are fully deprotonated and also undergo pure 
electron transfer. In the $\mathrm{pH}$ region between the two $\mathrm{pK}_{\mathrm{a}} \mathrm{s}$, the system undergoes a proton coupled electron transfer predicted by Equation 3.2.

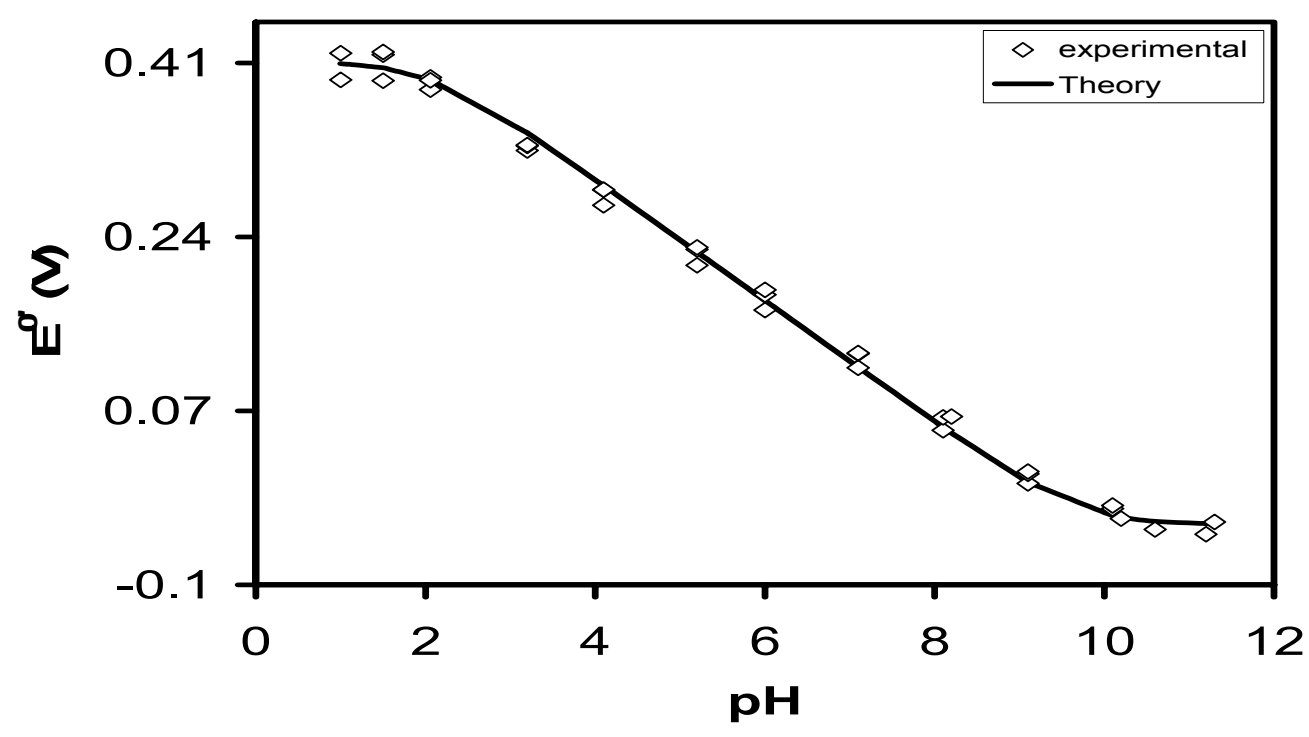

(a).

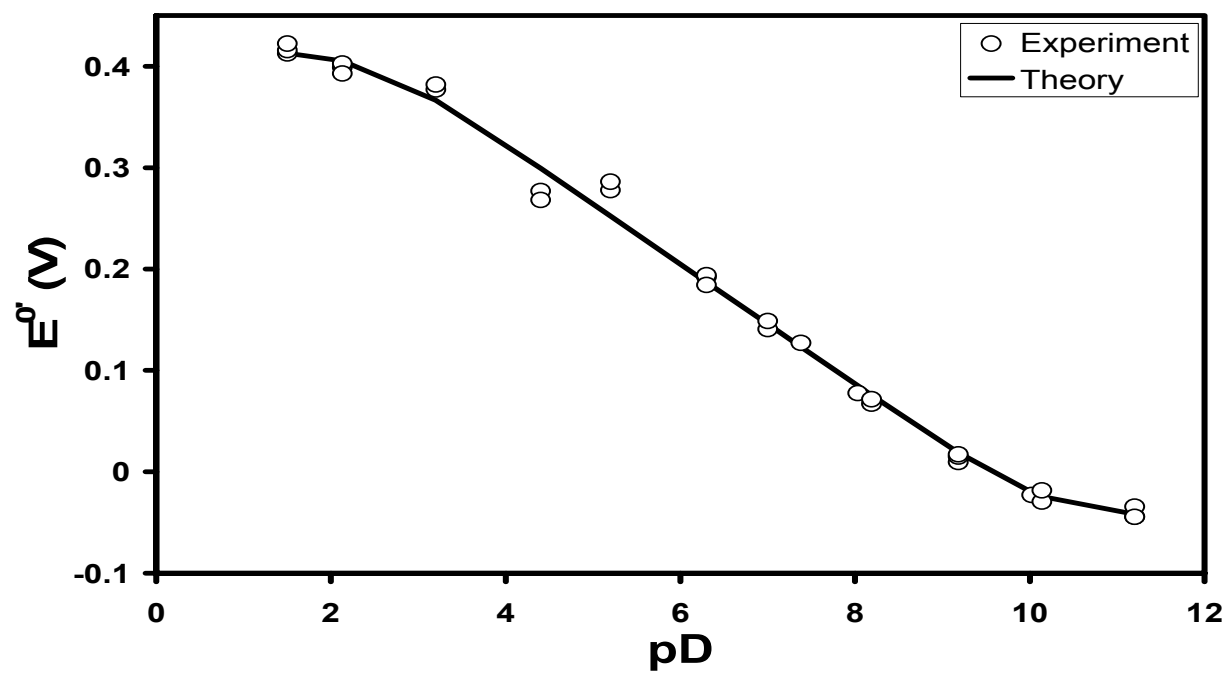

(b).

Figure 6.2 Variation of formal potential vs $\mathrm{Ag} / \mathrm{AgCl}$ with $\mathrm{pH}$ (a) or $\mathrm{pD}(\mathrm{b})$. Symbols represent experimental data based on three different experiments. The line represent a theoretical fit based on Equation 3.2. 
The experimental data were fit to theory using Equation 3.2 to obtain the four thermodynamic parameters: $\mathrm{E}_{1}^{0}, \mathrm{E}_{2}^{0}, \mathrm{pK}_{\mathrm{a} 1}$ and $\mathrm{pK}_{\mathrm{a} 2}$. Table 6.1 summarizes the thermodynamic values for the osmium system in both $\mathrm{H}_{2} \mathrm{O}$ and $\mathrm{D}_{2} \mathrm{O}$. These values are compared to values obtained when the redox molecule is dissolved in solution. Within the limits of experimental uncertainty, the thermodynamic parameters for the surfaceconfined redox molecule compare well with those obtained for the redox molecule dissolved in solution. Also listed in Table 6.1 is data from previous work by Haddox ${ }^{22}$ on a thinner SAM $\left(\mathrm{HS}\left(\mathrm{CH}_{2}\right)_{12} \mathrm{OH}\right)$ to highlight the effect of chain length on the thermodynamic properties of the osmium system. There is good agreement between data obtained by Haddox and data obtained from this work, within experimental uncertainty. However, Haddox's data showed that the separation between $\mathrm{pK}_{\mathrm{a} 1}$ and $\mathrm{pK}_{\mathrm{a} 2}$ is slightly smaller than observed in this work. It is possible that the shorter chain used by Haddox could result in a slightly different kind of interaction between the osmium and the SAM than that experienced with a long SAM. This interaction could enhance the acidic properties of the second proton for the redox molecule. However, other data sets indicate that $\mathrm{pK}_{\mathrm{a}}$ values have an uncertainty of at least \pm 0.2 . and this could mean that the $\mathrm{pK}_{\mathrm{a}}$ values are within experimental uncertainty. It is possible that, to a first approximation, the $\mathrm{pK}_{\mathrm{a}}$ values are independent of chain length. It is also important to note that, in solution, the pendant amine group on the redox molecule has a $\mathrm{pK}_{\mathrm{a}}$ near $\mathrm{pK}_{\mathrm{a} 2}$, which might interfere with the accurate determination of $\mathrm{pK}_{\mathrm{a} 2}$.

Although the formal potentials did not change significantly between heavy water and light water, the $\mathrm{pK}_{\mathrm{a}} \mathrm{s}$ obtained in heavy water were slightly shifted to higher values. The differences could be due to the inherent differences between the hydrogen and 
deuterium isotopes. Since the glass $\mathrm{pH}$ electrode used to measure $\mathrm{pH}$ was calibrated in light water in both cases, the actual $\mathrm{pH}$ reading might be slightly shifted in heavy water compared to regular water.

Because the deuteron has a heavier nucleus than the proton, its activity is different from that of the proton. Consequently, it sets up a slightly different junction potential between the glass membrane and the contacting solution than that set up by the proton. ${ }^{76}$ This difference in the potential results in a shift in measured $\mathrm{pH}$ which requires a correction factor. Several authors have reported addition of a correction factor of 0.4 to the $\mathrm{pH}$ reading obtained in heavy water to correct it to the regular water scale. ${ }^{73-75}$ However, in a recent study, Kretzel and $\mathrm{Bal}^{76}$ observed that $\mathrm{pK}_{\mathrm{a}} \mathrm{s}$ measured in $\mathrm{D}_{2} \mathrm{O}$ were higher than those measured in $\mathrm{H}_{2} \mathrm{O}$. Based on their analysis, they suggested a modification to the original formulation, see Equation 5.5. This linear correlation formula was used to correct the $\mathrm{pK}_{\mathrm{a}}$ values for the deuterium isotope in this work. Even after applying the correction factor (last column of Table 6.1), $\mathrm{pK}_{\mathrm{a}}$ values obtained in deuterium oxide remain slightly higher, but within experimental uncertainty of the $\mathrm{pK}_{\mathrm{a}} \mathrm{s}$ obtained in light water. 


\begin{tabular}{|l|l|l|l|l|l|l|}
\hline Solvent & $\mathrm{SAM}^{\mathrm{a}}$ & $\mathrm{pK}_{\mathrm{a} 1}$ & $\mathrm{pK}_{\mathrm{a} 2}$ & $\mathrm{E}_{1}^{0}{ }^{\mathrm{b}}$ & $\mathrm{E}_{2}^{0}{ }^{\mathrm{b}}$ & Ref. \\
\hline $\mathrm{H}_{2} \mathrm{O}$ & none & 1.96 & 9.73 & +0.32 & -0.13 & this work \\
\hline $\mathrm{H}_{2} \mathrm{O}$ & thinner & 2.4 & 9.3 & +0.34 & -0.07 & $\begin{array}{l}{[\text { Haddox }} \\
\text { 2004] }\end{array}$ \\
\hline $\mathrm{H}_{2} \mathrm{O}$ & thicker & 2.05 & 9.70 & +0.41 & -0.04 & this work \\
\hline $\mathrm{D}_{2} \mathrm{O}$ & thicker & 2.44 & 10.21 & +0.42 & -0.04 & this work \\
\hline $\mathrm{D}_{2} \mathrm{O}^{\mathrm{c}}$ & thicker & 2.67 & 9.90 & & & \\
\hline
\end{tabular}

Table 6.1: Thermodynamic parameters for the $\left[\mathrm{Os}{ }^{\mathrm{II} / \mathrm{III}}(\mathrm{bpy})_{2}(\mathrm{py})(\mathrm{L})\right]\left(\mathrm{L}=\mathrm{OH}^{-}\right.$or $\left.\mathrm{H}_{2} \mathrm{O}\right)$ complex.

${ }^{\mathrm{a}}$ Thinner self-assembled monolayers contained $\mathrm{HS}\left(\mathrm{CH}_{2}\right)_{15} \mathrm{COOH}$ and $\mathrm{HS}\left(\mathrm{CH}_{2}\right)_{12} \mathrm{OH}$, thicker SAMs contained $\mathrm{HS}\left(\mathrm{CH}_{2}\right)_{15} \mathrm{COOH}$ and $\mathrm{HS}\left(\mathrm{CH}_{2}\right){ }_{16} \mathrm{OH}$. Values measured on a bare gold electrode for the complex dissolved in the electrolyte are shown on the first line. ${ }^{b}$ Formal potentials with respect to the $\mathrm{Ag} / \mathrm{AgCl}$ reference electrode. ${ }^{\mathrm{c}} \mathrm{The}^{\mathrm{pK}} \mathrm{a}$ values measured in $\mathrm{D}_{2} \mathrm{O}$ are corrected to the water scale by applying a linear correlation reported by Kretzel and $\mathrm{Bal}^{75}$

Having measured the thermodynamic properties of the osmium system, the predictions of the stepwise and concerted models on the kinetic behavior of this system were investigated. Figure 6.3 (a) - (e) are typical CVs obtained at 0.1, 1.0, 10, 100 and $1000 \mathrm{~V} / \mathrm{s}$. CVs at 10, 100 and $1000 \mathrm{~V} / \mathrm{s}$ were under kinetic control and were used to obtain kinetic information; CVs at $1 \mathrm{~V} / \mathrm{s}$ are under mixed thermodynamic and kinetic control. 


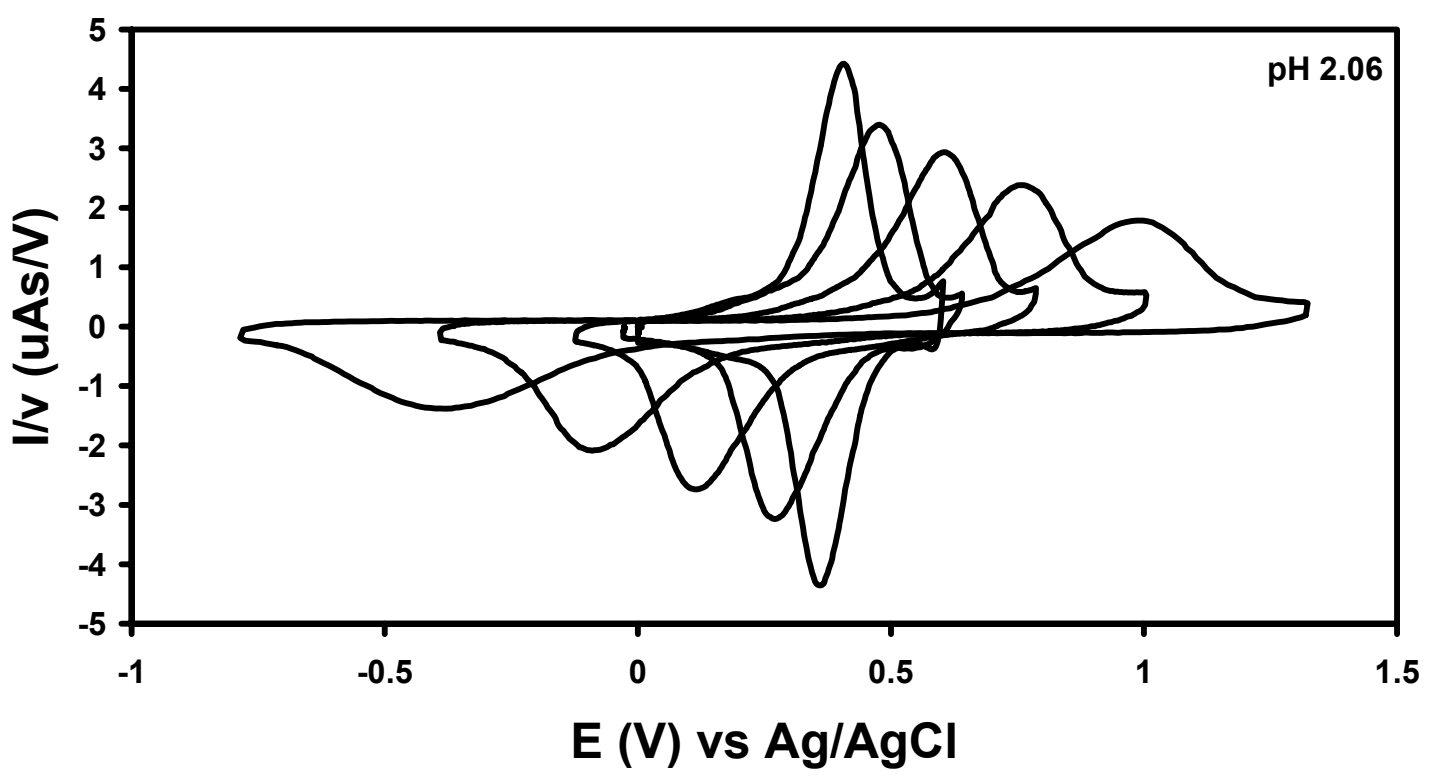

(a). Overlay CVs at pH 2.06.

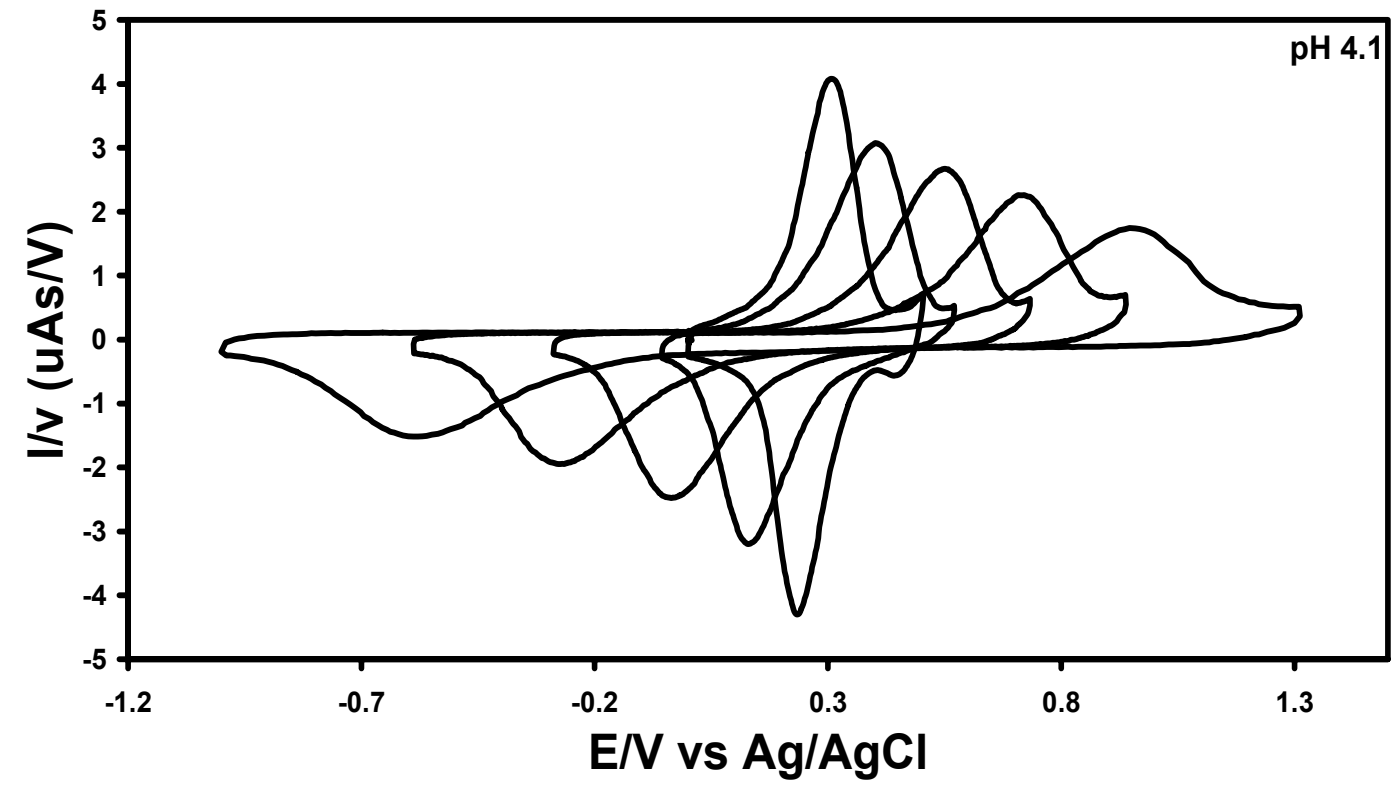

(b). Overlay CVs at pH 4.1. 


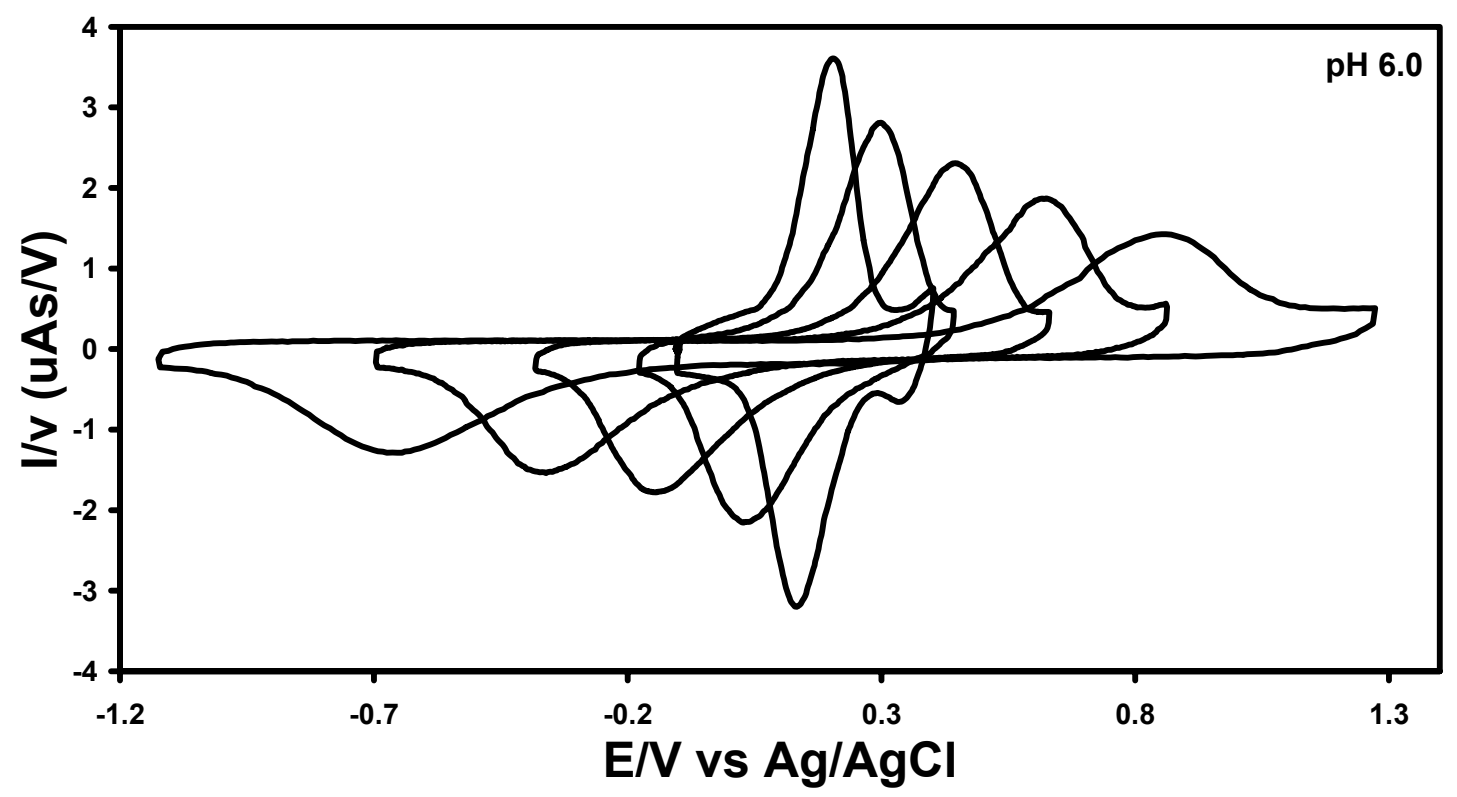

(c) Overlay CVs at pH 6.0.

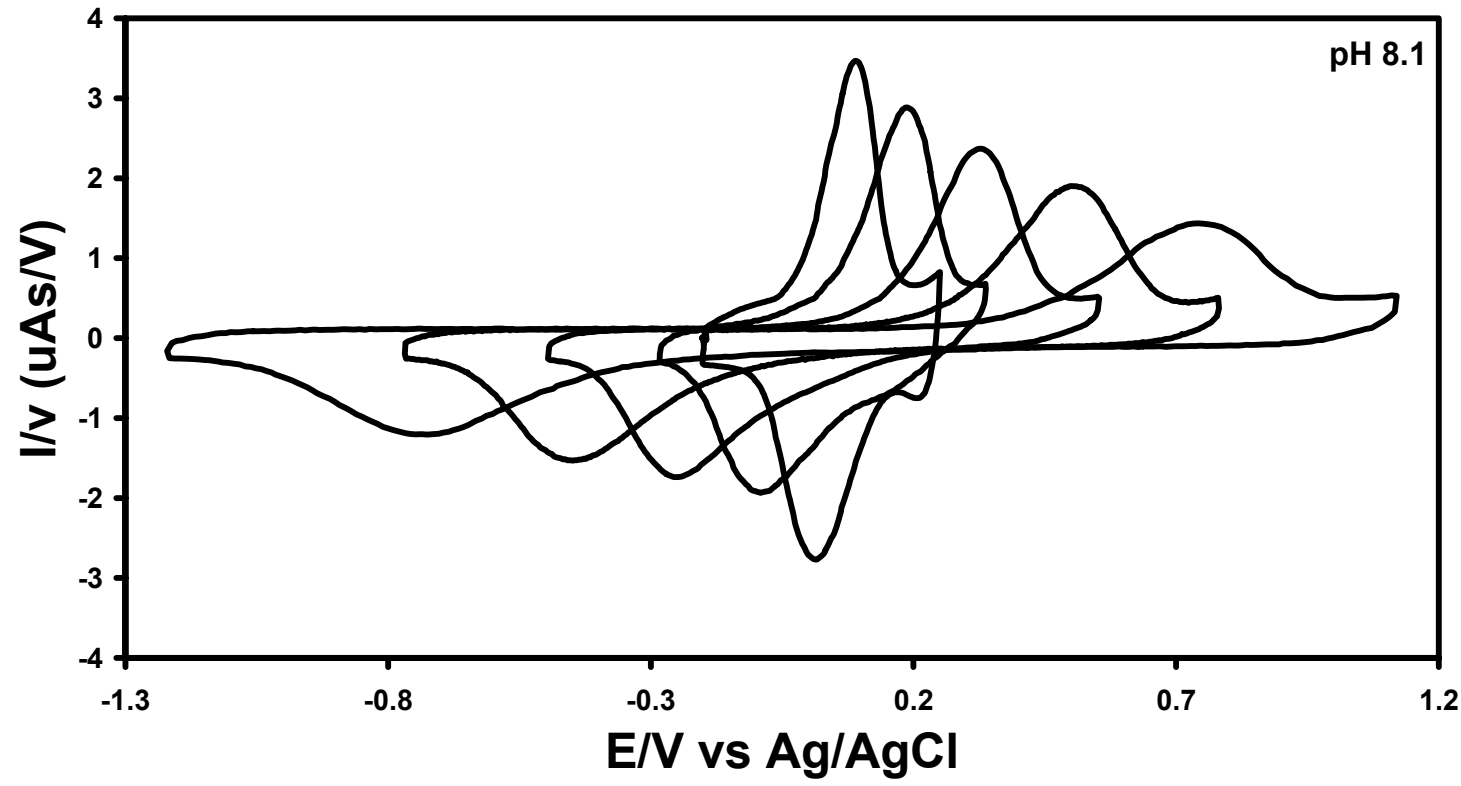

(d). Overlay CVs at pH 8.1. 


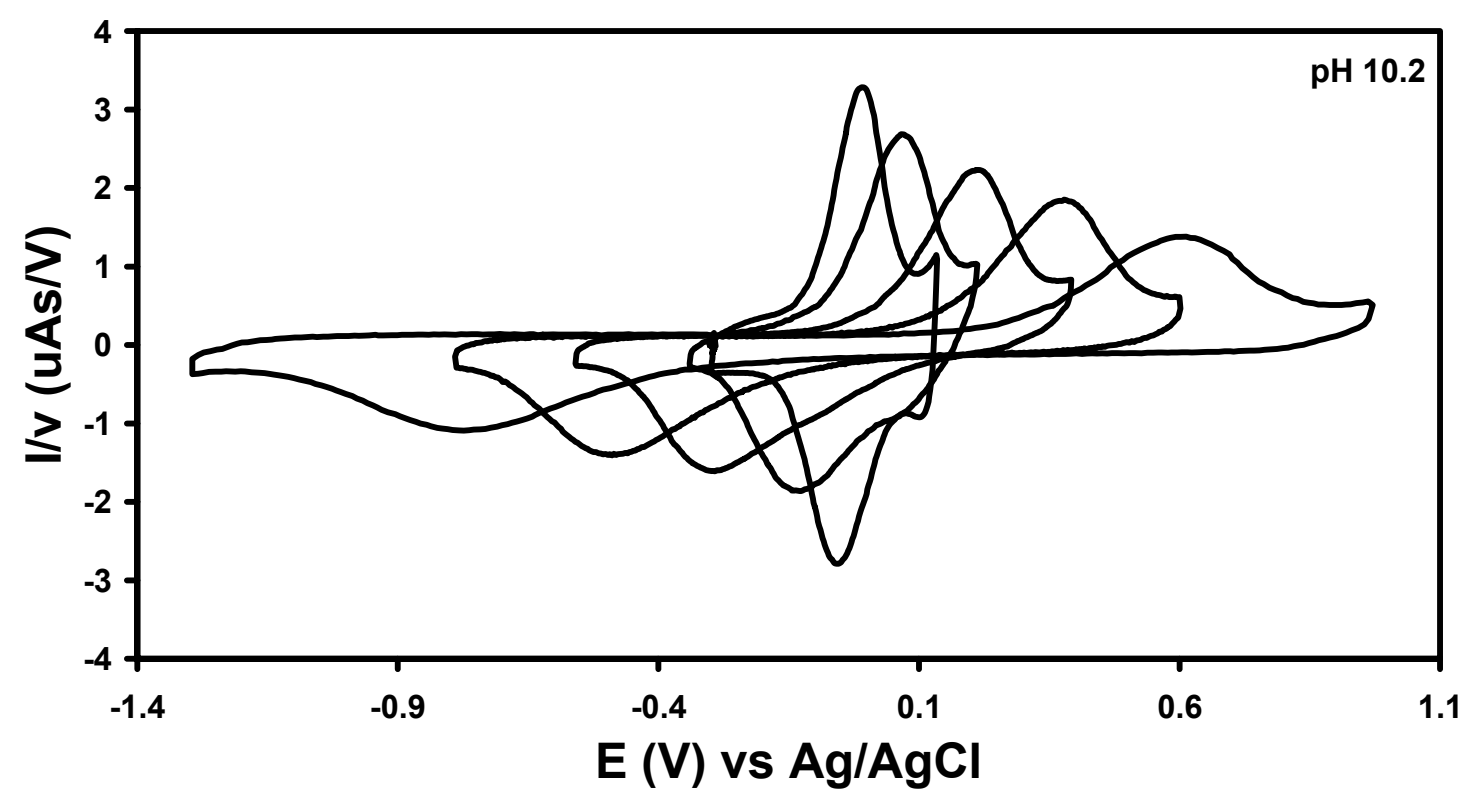

(e). Overlay CVs at $\mathrm{pH} 10.2$.

Figure 6.3. Overlay plots at selected pHs (a). $\mathrm{pH}$ 2.06, (b). $\mathrm{pH} 4.1$, (c). $\mathrm{pH}$ 6.0, (d) $\mathrm{pH} 8.1$ (d) $\mathrm{pH} 10.2$. Scan rates were recorded at $0.1,1,10,100$ and 1000V/s. Currents were normalized with respect to scan rate. The working electrode was a gold electrode with a redox center attached to it through a SAM. The electrolyte was $1.0 \mathrm{M} \mathrm{H}_{2} \mathrm{SO}_{4}$ in $0.1 \mathrm{M}$ Britton-Robinson Buffer adjusted to the indicated $\mathrm{pH}$ with $\mathrm{KOH}$. The reference electrode was an $\mathrm{Ag} / \mathrm{AgCl}$ chloride (Cypress).

These CVs can also act as a measure of the symmetry properties predicted by the stepwise model. As already discussed in Chapter 3, the shapes and symmetry of CVs were expected to vary with $\mathrm{pH}$. The $\mathrm{pH}$ ranges were carefully selected to compare the behavior predicted by the stepwise model in targeted regions of the $\mathrm{pH}$ scale. At $\mathrm{pH}$ lower than $\mathrm{pK}_{\mathrm{mid}}$, anodic $\mathrm{CV}$ are expected to be sharper and less sensitive to scan rate than the cathodic peaks, while at $\mathrm{pH}$ greater than $\mathrm{pK}_{\text {mid }}$, the cathodic peaks are expected to be sharper and less shifted with increasing scan rate. At mid $\mathrm{pH}$ and at extreme $\mathrm{pHs}$ 
where the transfer coefficient is equal to 0.5 , symmetric $\mathrm{CVs}$ are expected. The selected pHs cover the whole $\mathrm{pK}_{\mathrm{a}}$ spectrum; $\mathrm{pH} 2.06$ and $\mathrm{pH} 10.2$ representing the $\mathrm{pH}$ extremes. The mid $\mathrm{pK}_{\mathrm{a}}$ is 5.87. Symmetric CVs are expected around $\mathrm{pH}$ 6.0. Visual inspection suggest that the cathodic waves are slightly broader at all pHs. However, it is difficult to make a reliable comparison through visual inspection.

A more reliable method is to look at the shift of each peak potential as a function of scan rate. Figure 6.4 (a) to (e) are corresponding plots for each of the pHs in Figure 6.3 , showing the behavior of each peak potential as the scan rate was increased. The peak shift is calculated relative to the formal potential.

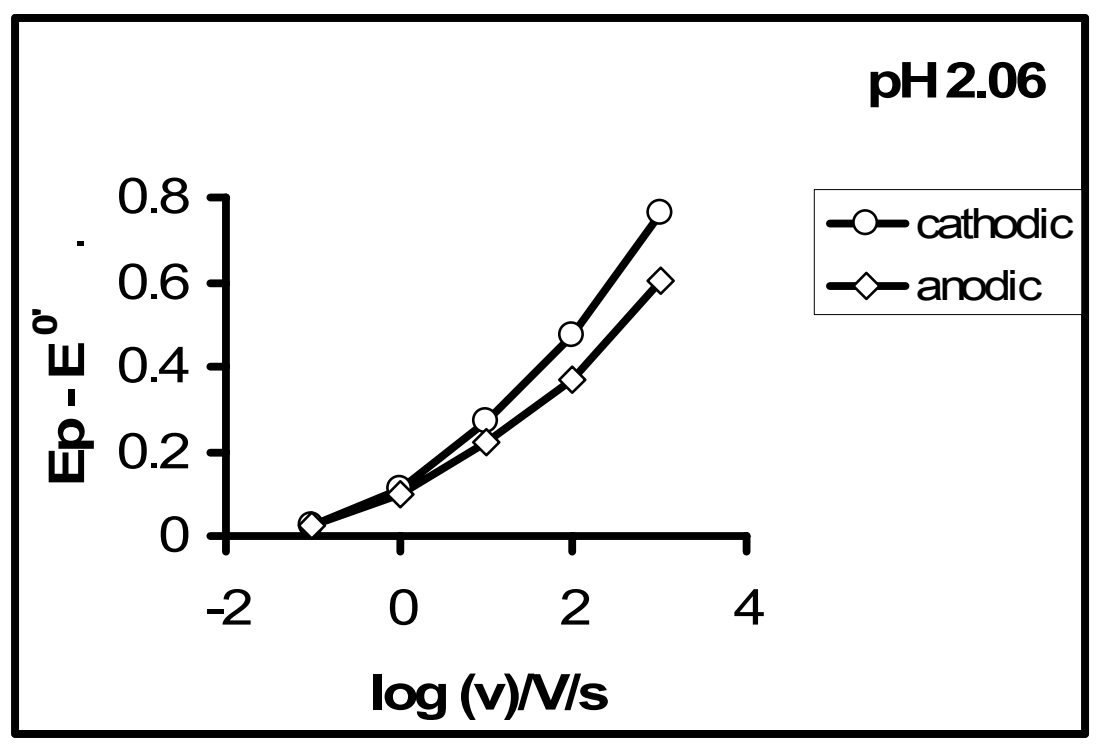

(a) 


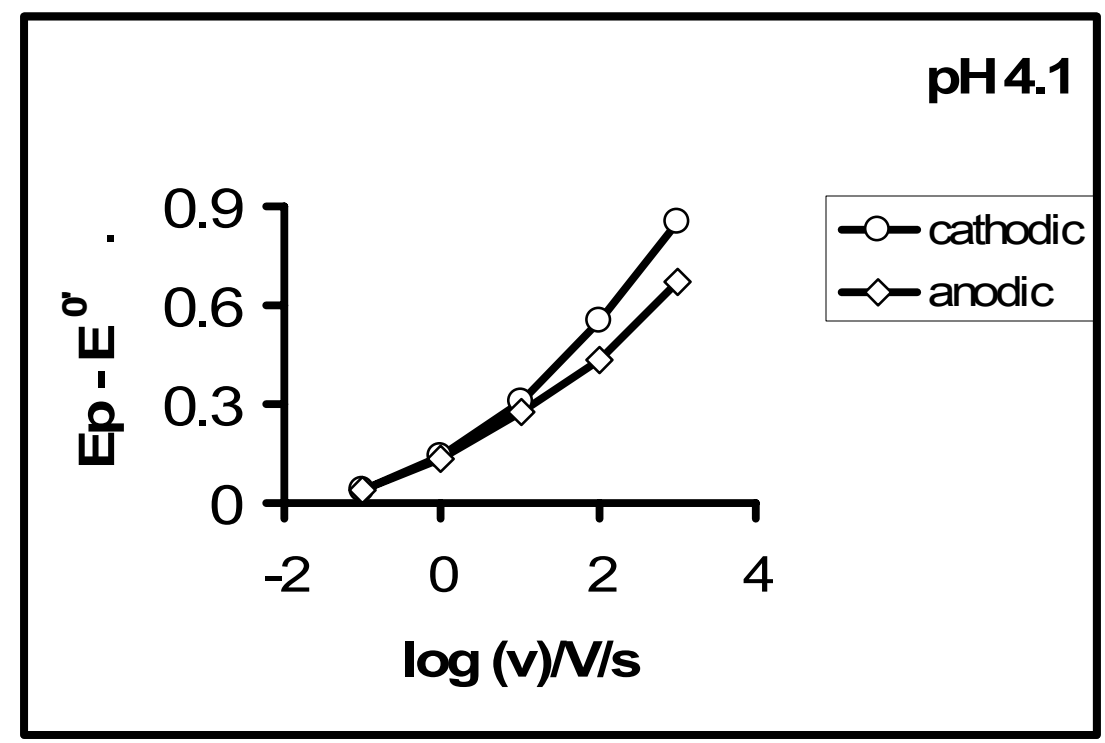

(b)

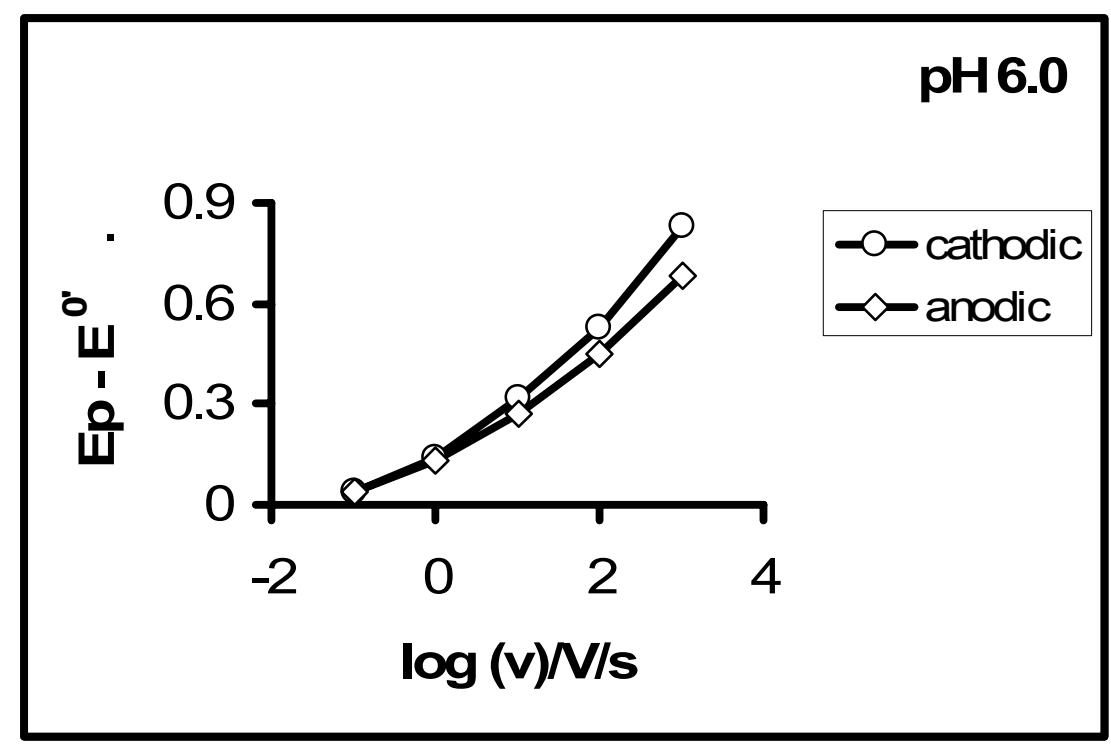

(c) 


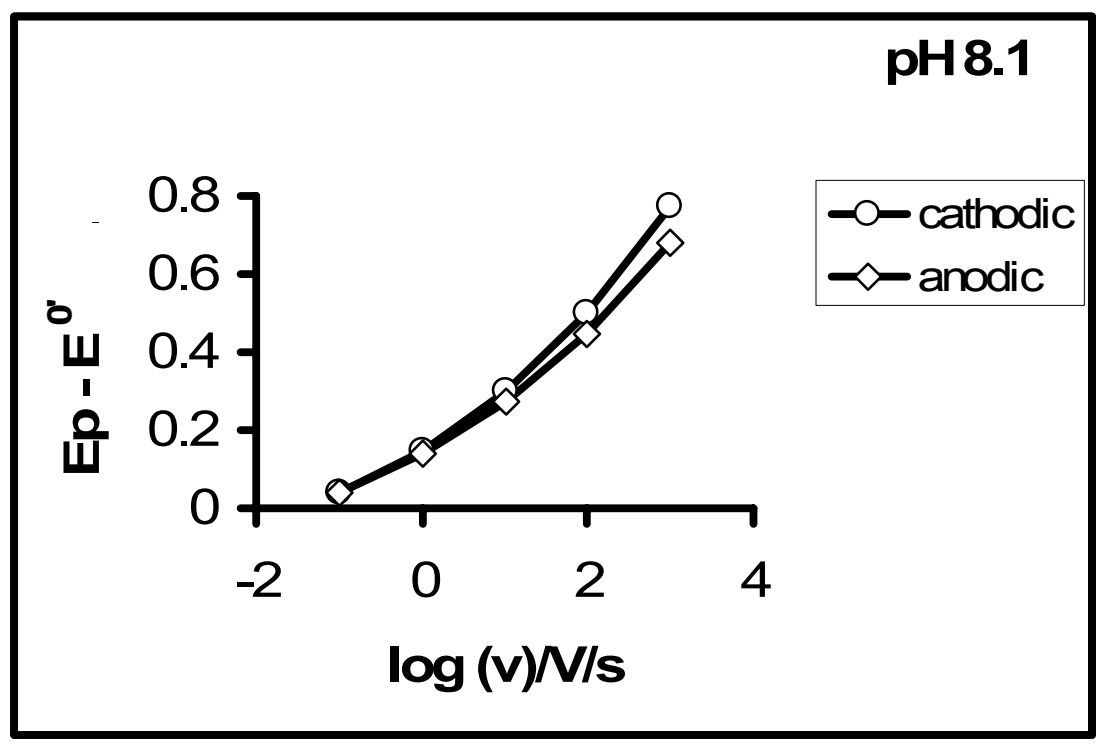

(d)

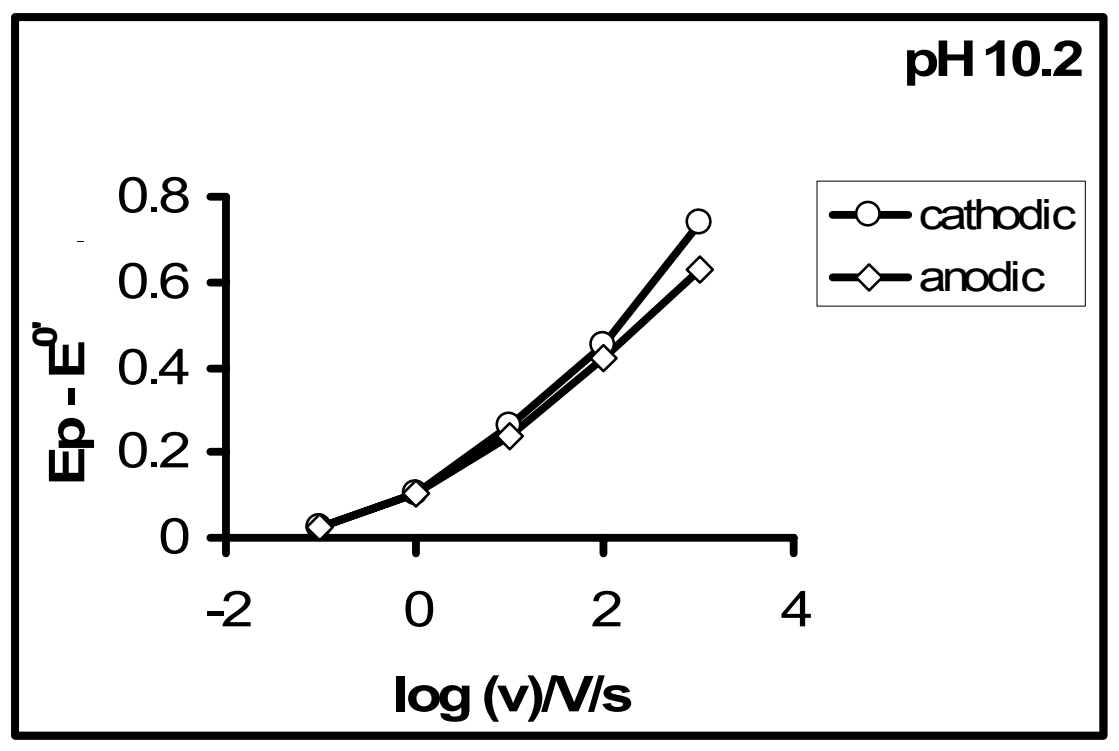

(e)

Figure $6.4(\mathrm{a})-(\mathrm{e})$. Variation of peak potential with scan rate at selected pHs. pHs were selected to highlight behavior at extreme $\mathrm{pH}$, mid $\mathrm{pH}, \mathrm{pK}_{\mathrm{a} 1}<. \mathrm{pH}<\mathrm{pK}_{\text {mid }}$ and $\mathrm{pK}_{\text {mid }}<. \mathrm{pH}$ $<\mathrm{pK}_{\mathrm{a} 2}$ 
At lower scan rates $(0.1-10 \mathrm{~V} / \mathrm{s})$, the peak shift is symmetrical at all $\mathrm{pHs}$. However at higher scan rates $(100-1000 \mathrm{~V} / \mathrm{s})$, the cathodic peak shift is higher (steeper slopes) than the anodic peak shift. This deviation is more pronounced at lower pHs than at higher $\mathrm{pHs}$. Clearly, these observations are not in line with the predictions discussed above.

One of the consequences of a potential dependent transfer coefficient used in Finklea's model is that Tafel plots $\left(\log k_{s} v s \eta\right)$ show curvature as predicted by Marcus density of states theory. Figure 6.5 shows a typical Tafel plot for the Os(bpy $\left.)_{2}(\mathrm{py}) \mathrm{OH}_{2}\right]^{2+}$ system generated at $\mathrm{pH} 6.0$.

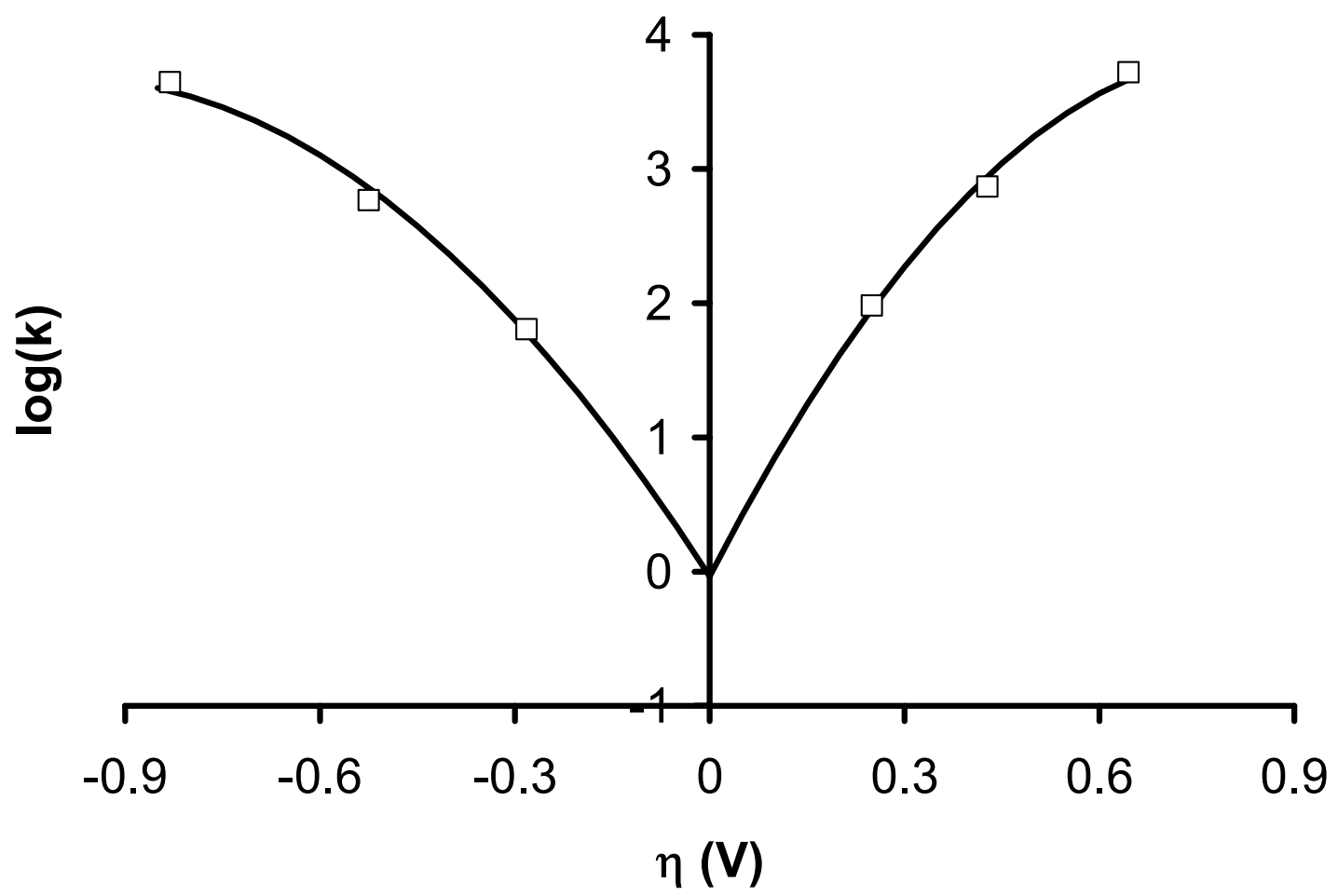

Figure 6.5. Typical Tafel Plot at $\mathrm{pH} 6.0$ for the $\left.\mathrm{Os}(\mathrm{bpy})_{2}(\mathrm{py}) \mathrm{OH}_{2}\right]^{2+}$ system. Note the asymmetry between the anodic and cathodic branch. The anodic branch rises more steeply than the cathodic branch. 
The symbols represent experimental data. The solid line is a theoretical fit obtained from functional dependence of alpha with respect to overpotential ( $)$. Figure 6.6 shows such a functional dependence. As described in Chapter 5, the alpha vs eta plot is fitted with separate linear regression lines for each branch and adjusting the rate constant to get a common intercept at alpha( 0 ). This yields the standard rate constant. This value is then substituted into a formula to calculate alpha values as a function of overpotential; $\log \left(\mathrm{k}_{\mathrm{s}}\right)$ is then calculated from alpha values using the slope and intercept from Figure 6.6 and plotted against the overpotential to generate Figure 6.5.

The curvature in the Tafel plot can be related to reorganization energy. Tafel plots can be used to estimate the reorganization by fitting experimental data to theoretical working curves. However, the reorganization energy can be conveniently obtained from Figure 6.6 as described in Chapter 5. From the slope of each branch, the reorganization energy can be calculated. Notice the difference in slopes for the two branches. 


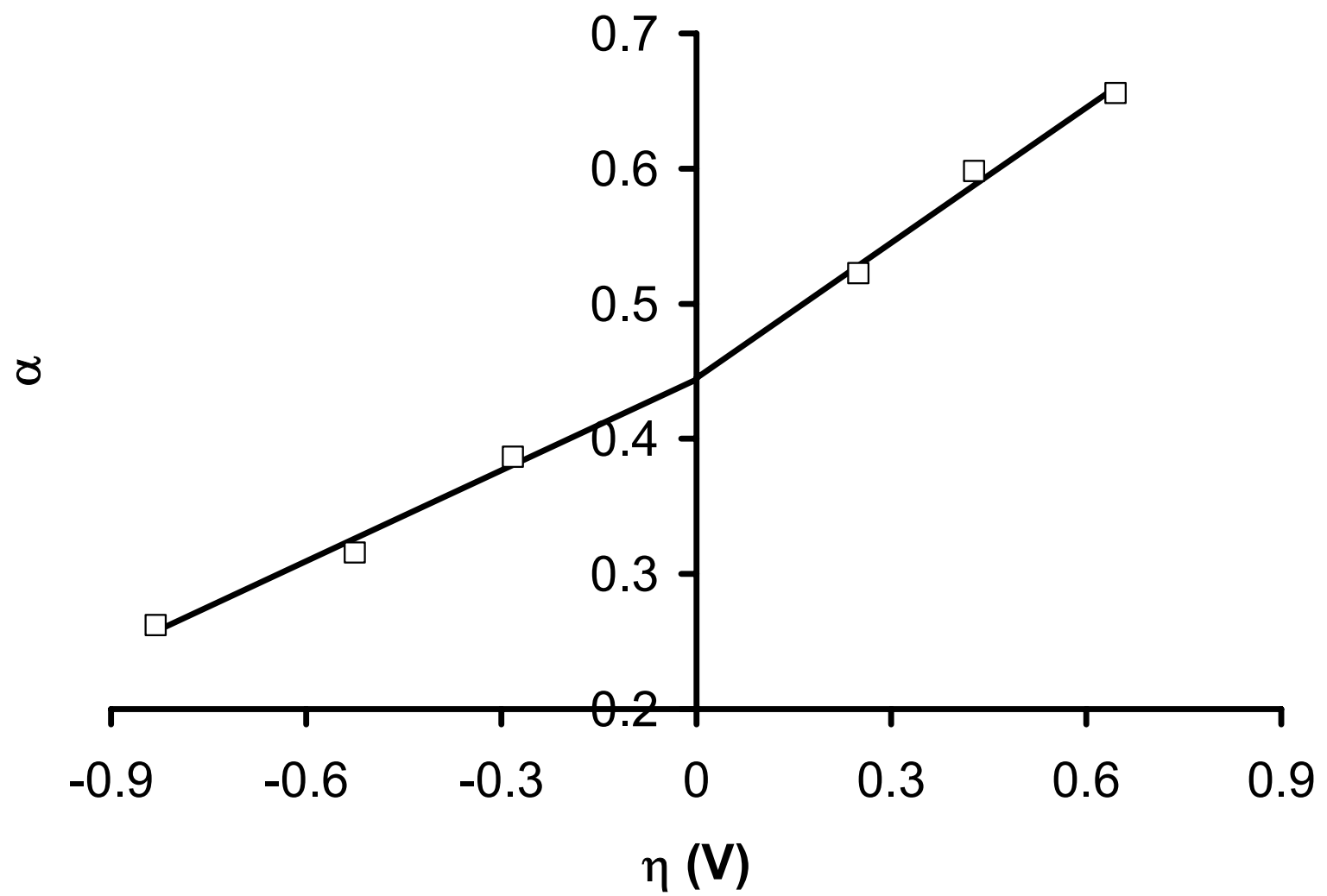

Figure 6.6. Potential dependence of the transfer coefficient at $\mathrm{pH} 6.0$ for the $\left.\mathrm{Os}(\mathrm{bpy})_{2}(\mathrm{py}) \mathrm{OH}_{2}\right]^{2+}$ system.

The anodic branch was steeper than the cathodic branch at all pHs. Although the two lines are fitted separately, they must have a common intercept to give a single value for the transfer coefficient at zero overpotential $(\alpha(0))$. We know of no experimental or theoretical arguments to have two separate transfer coefficient for the oxidized and reduced species.

Data in Figure 6.6 were fitted by a standard rate constant of $0.92 \mathrm{~s}^{-1}$, and yielded an anodic slope of $0.33 \pm 0.05 \mathrm{~V}^{-1}$ and a cathodic slope of $0.22 \pm 0.03 \mathrm{~V}^{-1}$. An alpha(0) of $0.44 \pm 0.02$ was obtained from the intercept. The slopes correspond to reorganization 
energies of $1.00 \pm 0.16 \mathrm{eV}$ for $\mathrm{Os}^{\mathrm{II}}$ and $0.64 \pm 0.10 \mathrm{eV}$ for $\mathrm{Os}^{\mathrm{III}}$. Similar plots were generated at each $\mathrm{pH}$ and used to extract all kinetic parameters described in this work. The cause of asymmetry in the Tafel plot (Figure.6.5) is related to different reorganization energies between the anodic and cathodic branch. The behavior of the transfer coefficient and Tafel plot shown in Figure 6.5 and 6.6 matches with predictions made by Finklea's derivation for different reorganization energies, Chapter 5 .

The standard rate constant was measured at each $\mathrm{pH}$ for the entire $\mathrm{pH}$ range and compared to theoretical predictions. Figure 6.7 compares the theoretical predictions (solid line) of the stepwise model with experiment (symbols) for the variation of the standard rate constant with $\mathrm{pH}$. Experimental data showed a weak dependence of the standard rate constant on $\mathrm{pH}$ contrary to predictions of the stepwise model. Although experimental data indicate that the standard rate constant decreases, passing through a minimum, as the $\mathrm{pH}$ of the contacting solution increases, the minimum is somewhat bow-shaped and is not as deep as predicted by the stepwise model. 


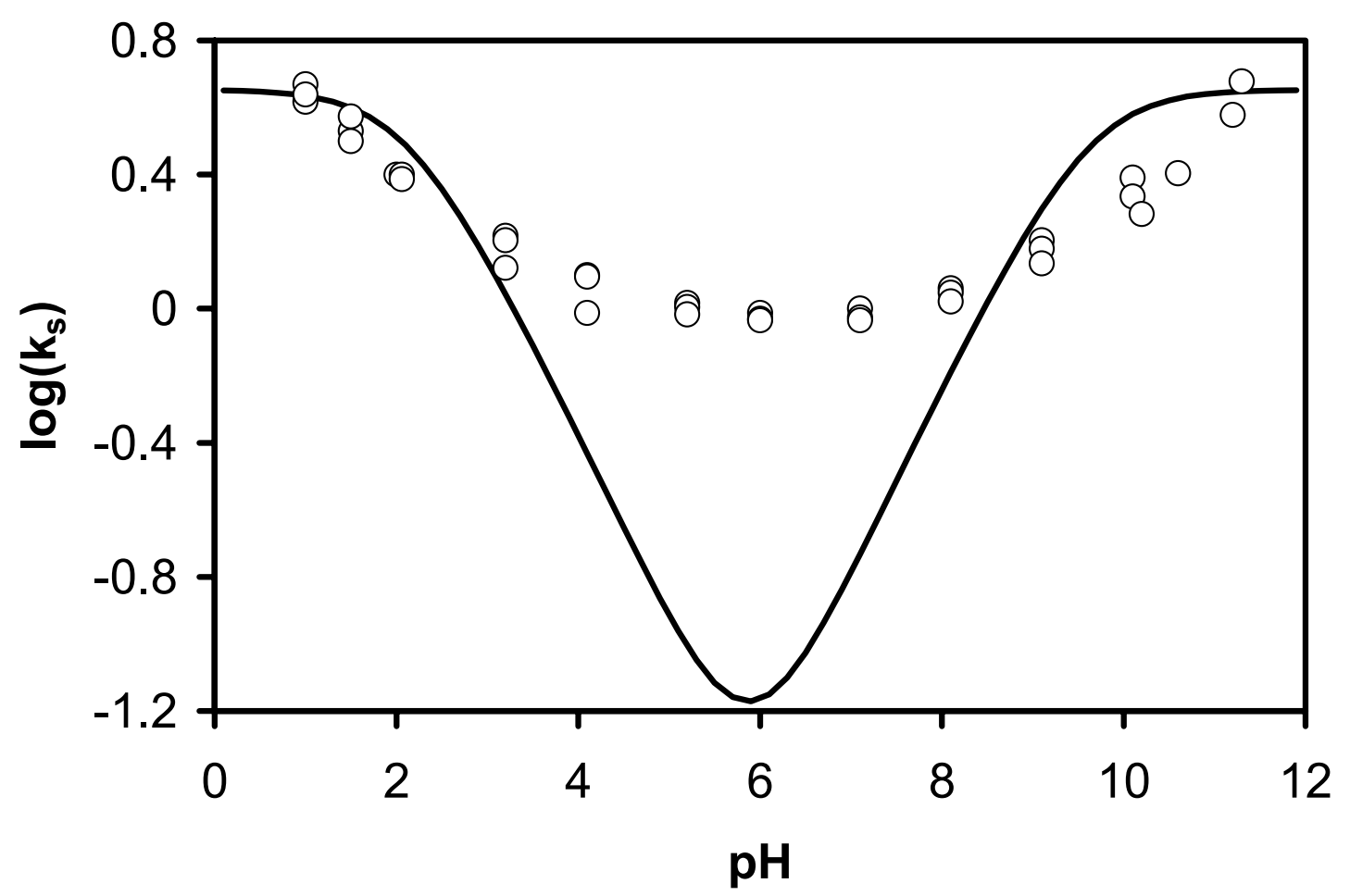

Figure 6.7. Comparison between experimental data (symbols) and theoretical predictions of the stepwise model (solid line). The data points represent three different experiments. The theoretical line was calculated using the following parameters: $\mathrm{E}_{1}^{0}=0.45 \mathrm{~V}, \mathrm{E}_{2}^{0}=0$ $\mathrm{V}, \mathrm{pK}_{\mathrm{a} 1}=2.05, \mathrm{pK}_{\mathrm{a} 2}=9.7, \mathrm{k}_{\mathrm{s} 1}=\mathrm{k}_{\mathrm{s} 2}=4.5 \mathrm{~s}^{-1}$ and $\lambda=0.9 \mathrm{eV}$.

Another striking feature is the absence of any break in the standard rate constant at extreme pHs as shown by the theoretical curve. As already discussed, this break signifies the onset of pure electron transfer independent of $\mathrm{pH}$ at $\mathrm{pHs}$ beyond the two $\mathrm{pK}_{\mathrm{a}}$ values. An earlier study by Haddox ${ }^{45}$ using a thinner SAM showed a weak $\mathrm{pH}$ dependence of the standard rate constant similar to that observed in this study. The kinetic behavior predicted by the stepwise model for the variation of the standard rate constant with $\mathrm{pH}$ could not be verified experimentally, in this study or in a study by 
Haddox. A reasonable conclusion from these observations is that the stepwise model cannot explain the kinetics observed in PCET reactions. This conclusion is further supported by comparison of $\alpha(0)$ vs $\mathrm{pH}$ with the stepwise model (section 6.1 ).

Another way to further investigate the stepwise mechanism is to look at the kinetic isotope effect which is the ratio of the rate constant in water compared to that in deuterium oxide. Figure 6.8 compares the standard rate constant obtained in regular buffers (squares) to those obtained in deuterated buffers(triangles) as a function of $\mathrm{pH}$ or $\mathrm{pD}$ ( $\mathrm{pH}$ in deuterated buffers). It is clear that the standard rate constant also shows a weak dependence on $\mathrm{pD}$ just like in water. However, the standard rate constant is slightly more sensitive to $\mathrm{pD}$ than $\mathrm{pH}$ giving an apparent kinetic isotope effect close to 2, within experimental uncertainty, above $\mathrm{pH} 3$. 


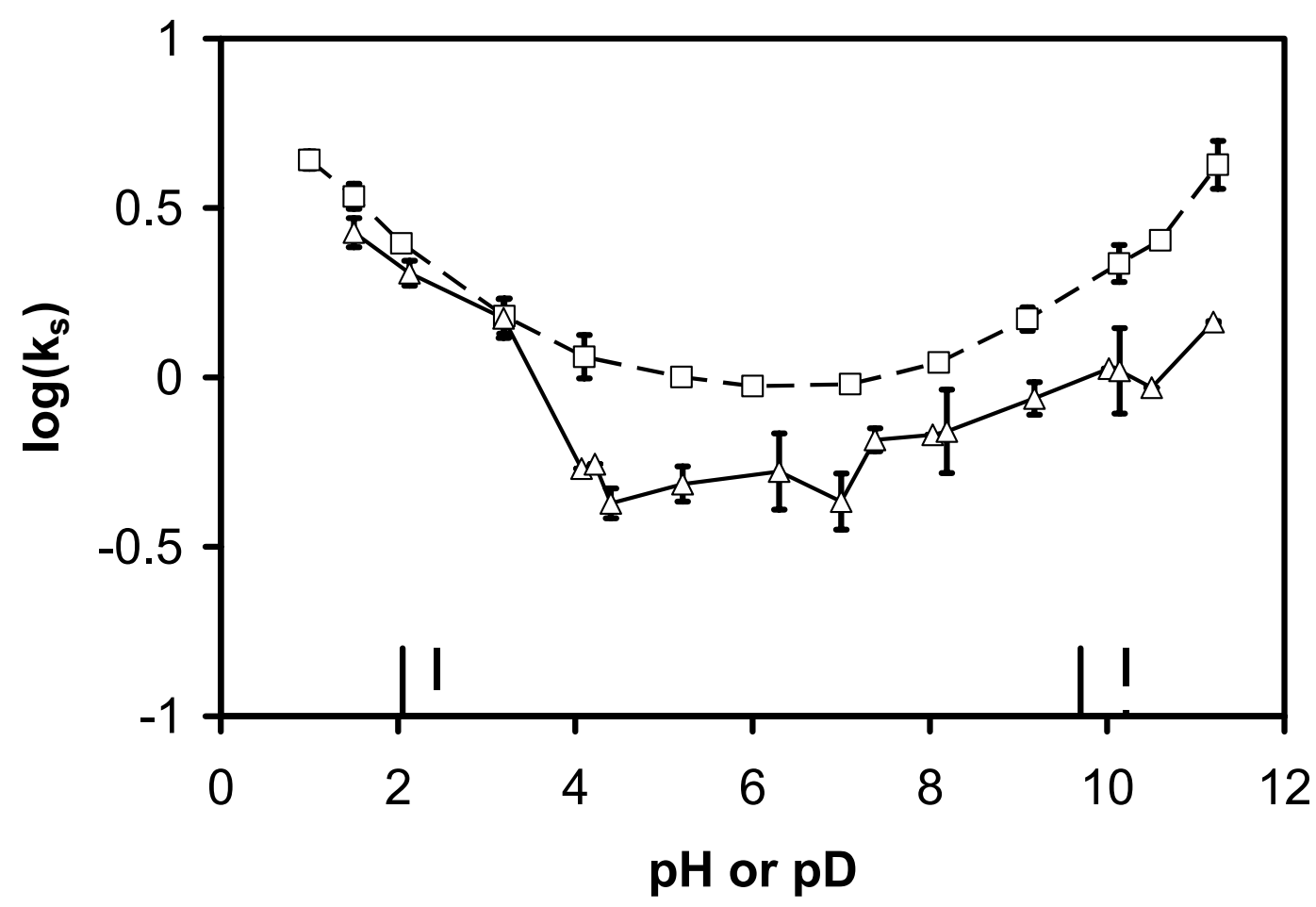

Figure 6.8. Kinetic Isotope effect: $\log \left(\mathrm{k}_{\mathrm{s}}\right)$ vs $\mathrm{pH}$ for $(90 / 10) \mathrm{HS}\left(\mathrm{CH}_{2}\right)_{15} \mathrm{COOH} /$ $\mathrm{HS}\left(\mathrm{CH}_{2}\right)_{16} \mathrm{OH}$ in $\mathrm{H}_{2} \mathrm{O}$ (squares) and $\mathrm{D}_{2} \mathrm{O}$ (triangles) Each data point is an average of three data sets. The bars represent one standard deviation. Two pairs of $\mathrm{pK}_{\mathrm{a}} \mathrm{s}$ are also shown by vertical bars on the $\mathrm{pH}$ scale. For each pair, the low $\mathrm{pK}_{\mathrm{a}}$ is for $\mathrm{H}_{2} \mathrm{O}$ while the higher $\mathrm{pK}_{\mathrm{a}}$ is for $\mathrm{D}_{2} \mathrm{O}$. Lines joining the points are a guide for the same set of data points.

In the stepwise model, proton transfer is assumed to be at equilibrium. Based on this assumption, the standard rate constant should not be affected by replacing the proton with the deuteron. However, if the concerted mechanism is controlling the rate, the standard rate constant is expected to be sensitive to the deuteron since the proton is part of the rate determining step of the concerted mechanism. Although the kinetic isotope effect is small, the decrease in the standard rate constant in deuterated electrolytes favors the concerted mechanism more than the stepwise mechanism. 
A startling observation in Figure 6.8 is the sudden break in the standard rate constant between $\mathrm{pD} 3$ and 4 . Replicate experiments confirmed that this break is real rather than just an experimental artifact. The cause of this break is not clear. It is tempting to think that since this break occurs close to $\mathrm{pK}_{\mathrm{a}}$, it could signal the onset of a PCET (a change in mechanism from pure electron transfer mechanism), but, a corresponding break was not observed in water, so this hypothesis could not be validated.

\subsubsection{Transfer coefficient vs $\mathrm{pH}$.}

The predictions of the stepwise model for the variation of the transfer coefficient with $\mathrm{pH}$ has already been discussed in Chapter 3. Figure 6.9 shows the observed behavior of the transfer coefficient as the $\mathrm{pH}$ of the contacting solution changes. Again, the $\mathrm{pK}_{\mathrm{a}}$ values are shown as vertical bars on the $\mathrm{pH}$ axis. The $\alpha(0) \mathrm{vs} \mathrm{pH}$ plot was expected to oscillate about 0.5 , that is, to be lower than 0.5 below mid $\mathrm{pH}$ and greater than 0.5 above mid $\mathrm{pH}$, then equal to 0.5 at mid $\mathrm{pH}$ and at the $\mathrm{pH}$ extremes, as shown by the solid line in Figure 6.9. None of this behavior is observed experimentally. Rather, Figure 6.9 shows that $\alpha(0)$ is weakly dependent on $\mathrm{pH}$ and is consistently below 0.5 at all $\mathrm{pHs}$. 


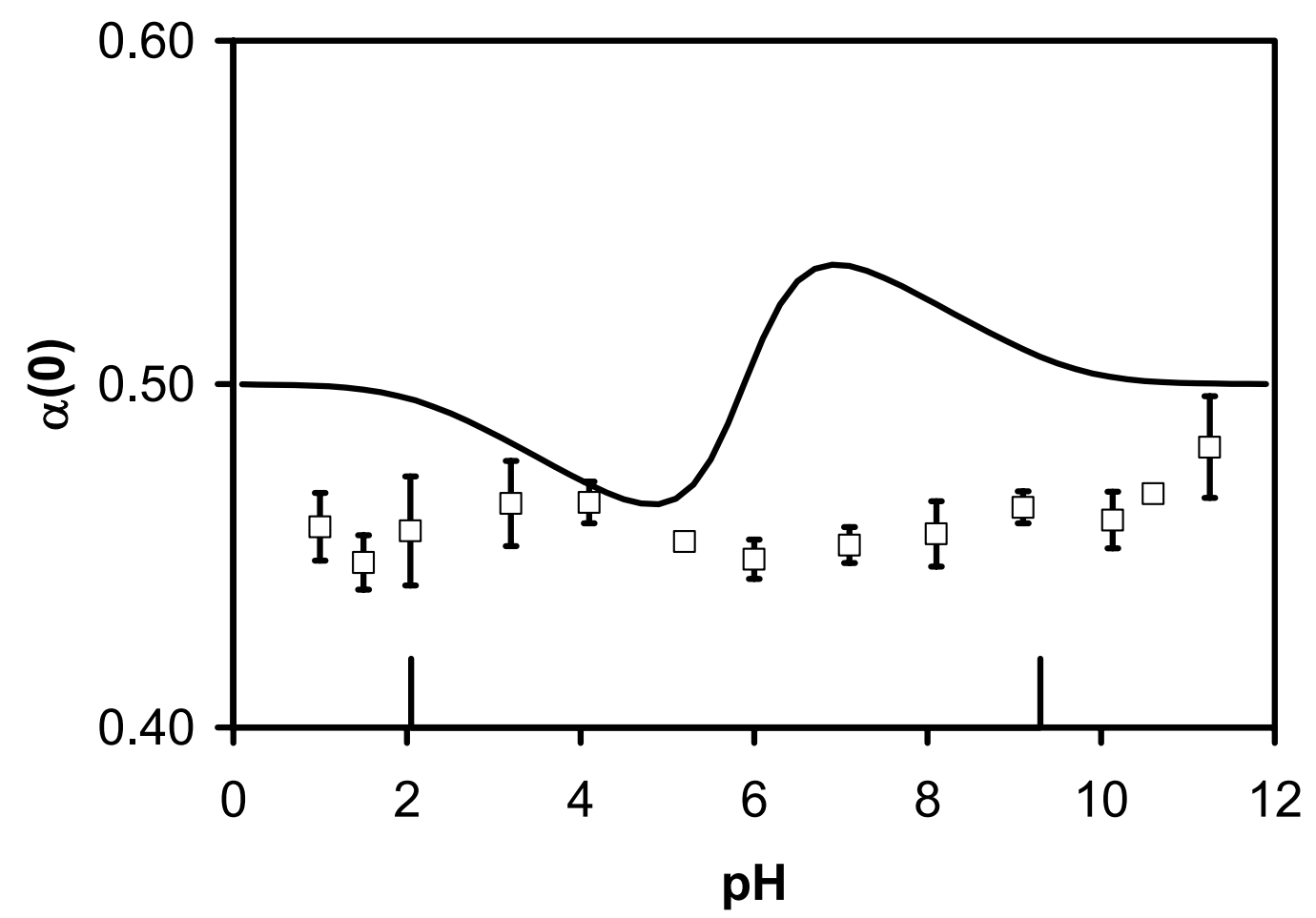

Figure 6.9.Variation of the transfer coefficient at zero overpotential as a function of $\mathrm{pH}$ in water. Symbols are the average values of three experiments, and bars represent one standard deviation. The $\mathrm{pK}_{\mathrm{a}}$ values are marked on the $\mathrm{pH}$ axis. The solid line is the theoretical prediction of the stepwise model.

Average $\alpha(0)$ values range from a minimum of 0.44 to a maximum of 0.48 at all pHs. This range agrees closely with Haddox data. ${ }^{45}$ Clearly, these observations do not conform to the stepwise model and a new theoretical model is required to explain these observations. 


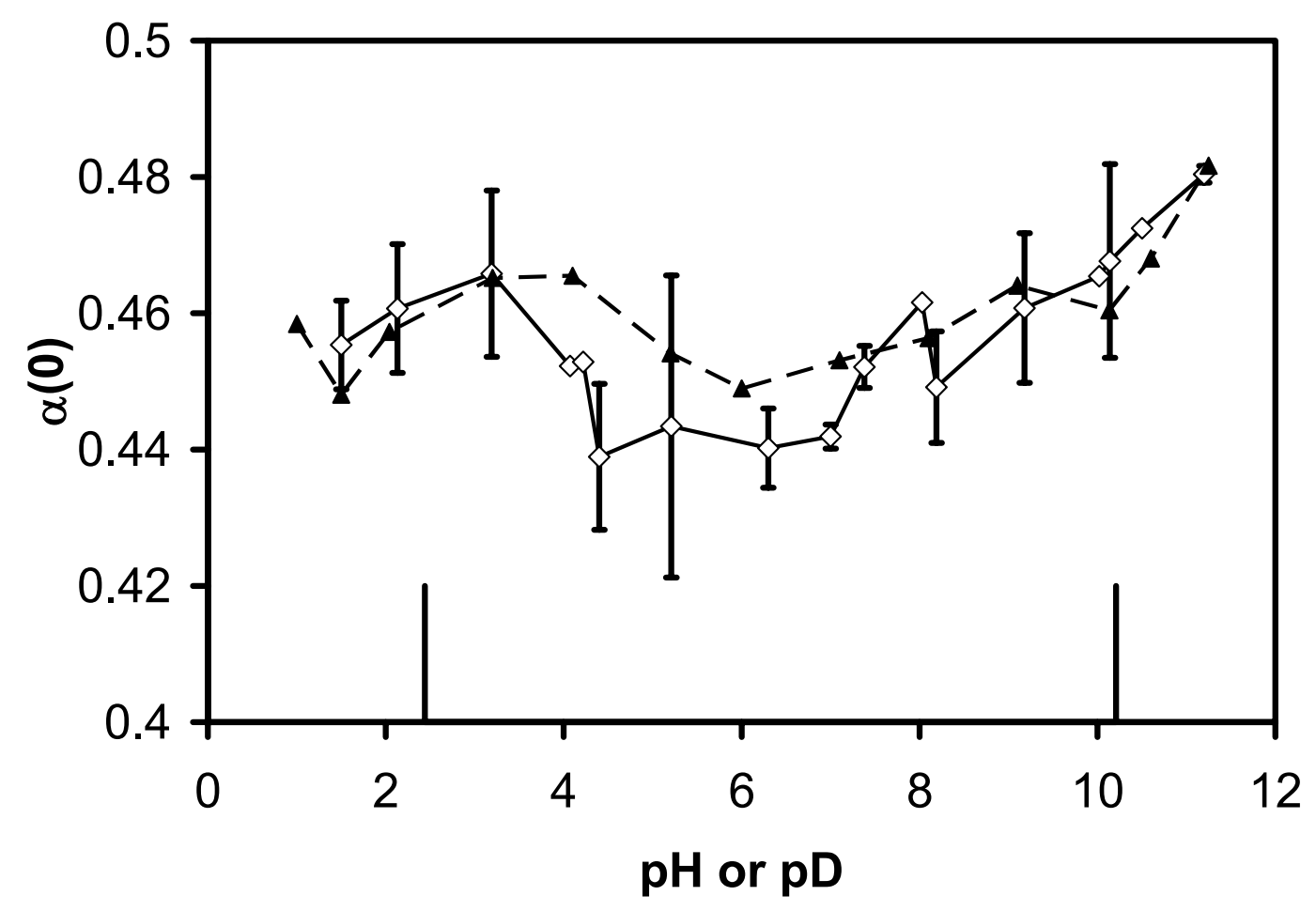

Figure 6.10. Comparing the transfer coefficients at zero overpotential for $\mathrm{H}_{2} \mathrm{O}$ (triangles) and $\mathrm{D}_{2} \mathrm{O}$ (diamonds). For $\mathrm{D}_{2} \mathrm{O}$, the symbols are an average of two or three experiments and the bars represent one standard deviation.

The data in Figure 6.9 are compared to similar data obtained in deuterated electrolytes in Figure 6.10. Although the data from deuterated electrolytes have a large scatter, on average, the transfer coefficient follows a similar trend observed in water. It is not possible to make a definitive conclusion about an isotope effect here due to the large standard deviations in the data. 


\subsubsection{Standard rate constant vs chain length.}

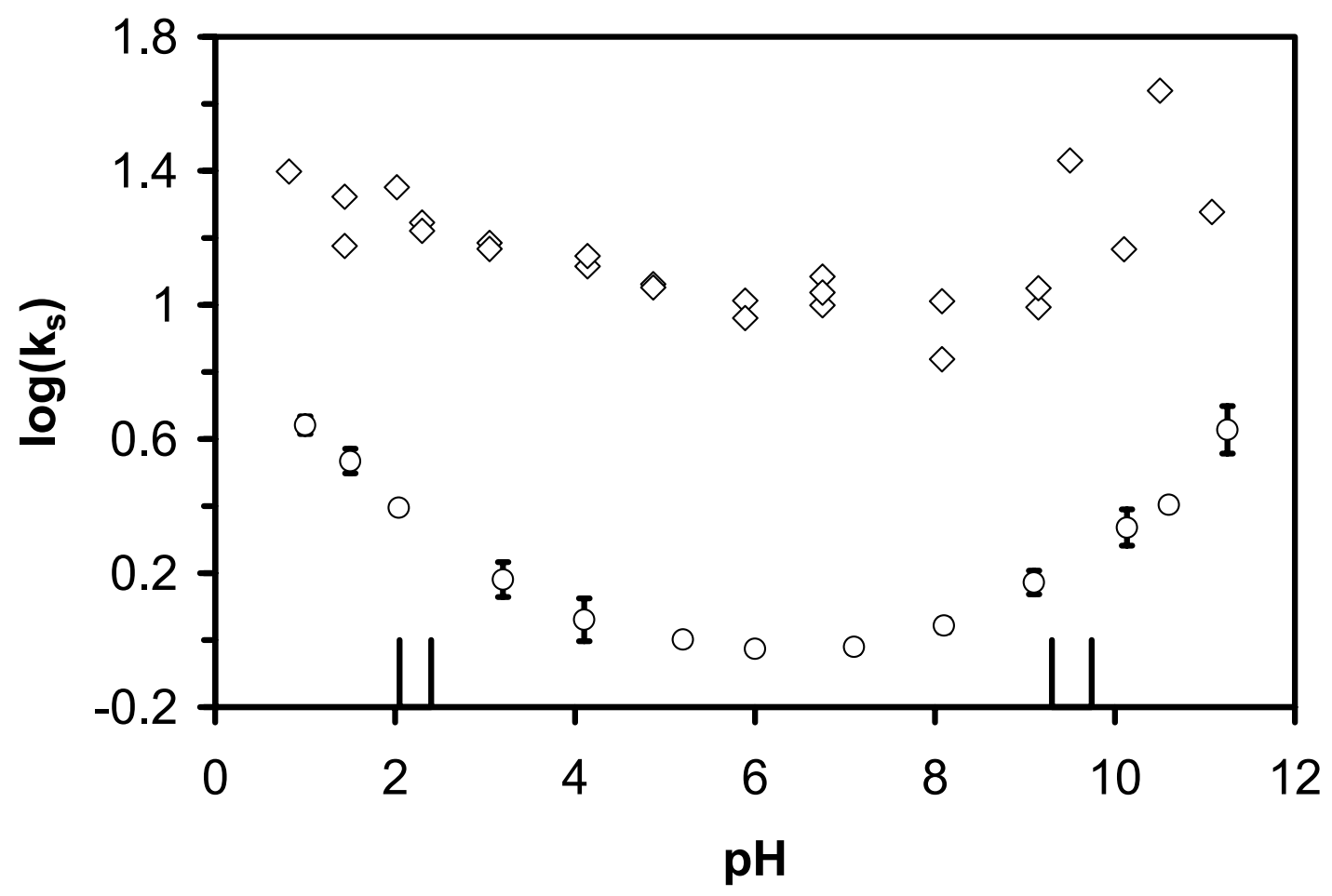

Figure 6.11. Effect of chain length on the standard rate constant in light water. Diamonds represent the data reported by Haddox,${ }^{45}$ with a thinner SAM. Circles represent the average standard rate constants (this work) for the data in Figure. 6.7; bars are one standard deviation. At several pHs, the spacing between the high and low bar is less than the size of the symbol.

The effect of chain length on the standard rate constant is investigated in Figure 6.11, by comparing data obtained on a thicker SAM (this work) to that obtained on a thinner SAM $\left(\operatorname{Haddox}^{45}\right)$. The standard rate constant decreases by a factor of 10 over the entire $\mathrm{pH}$ range when the thickness of the diluent monolayer was increased by four methylene groups. The dependence of the standard rate constant on chain length is evidence that the rate is more sensitive to electronic coupling between the osmium redox 
center and the electrode than it is to changes in $\mathrm{pH}$ of the contacting solution. At first, one would expect the rate constant to remain constant since the electroactive thiol has the same length in both cases. However, for the $\mathrm{C}_{12}$ case, the redox molecule is exposed and freely swinging on a short part of the tether. As a result, it is able to fold and come into contact with the terminal end of the diluent thiol for a period of time long enough for electron transfer to occur, Figure 6.11(a).

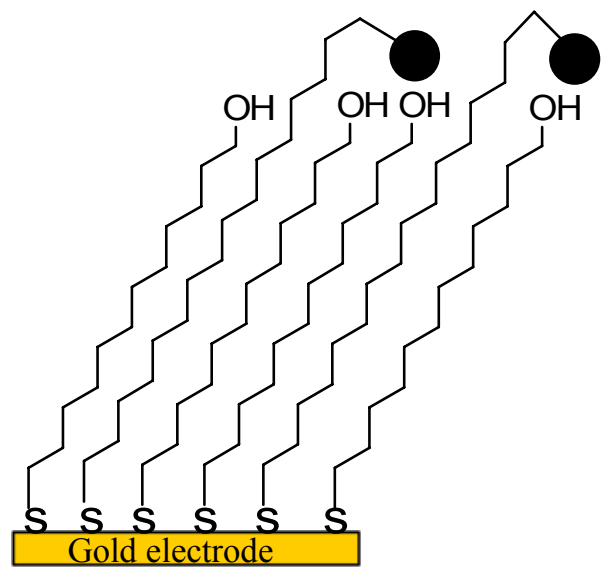

(a). Exposed redox molecules

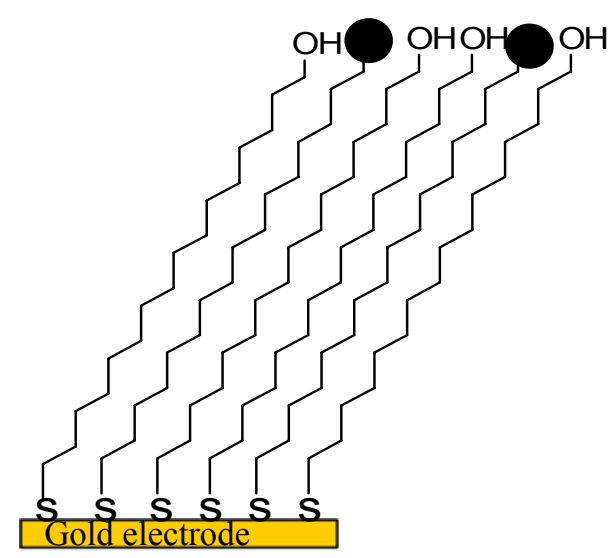

(b). Matched

Figure.6.12. Generic diagrams comparing the standard rate constant on different chain length diluent thiols. In each case, the electroactive chain has the same length. In the exposed case, the electroactive chain length is longer than the diluent chain. In the matched case, the diluent chain has the same length as the electroactive chain.

The electron tunneling distance is shorter and equal to the length of the $\mathrm{C}_{12}$ chain, leading to a higher rate of electron transfer. For the $\mathrm{C}_{16}$ chain, Figure 6.12 (b), the diluent chain matches the electroactive thiol in length. The electroactive chain cannot fold as shown in Figure 6.10 (a). The electron tunneling distance is equal to the length of the $\mathrm{C}_{16}$ 
chain which is longer than that for the $\mathrm{C}_{12}$ chain leading to lower rates of electron transfer.

The change in electronic coupling with respect to the increased length of the diluent thiol is consistent with the previous study by Finklea and others for exposed and matched chain lengths. ${ }^{35}$ In Finklea's study, $\mathrm{Ru}\left(\mathrm{NH}_{3}\right)_{5}(\mathrm{py})$ was used as a redox molecule and was connected to a $\mathrm{HSC}_{15} \mathrm{COOH} \mathrm{SAM}$ in both cases. In the matched case, the diluent thiols was $\mathrm{HSC}_{15} \mathrm{COOH}$, giving a standard rate constant of $1 \mathrm{~s}^{-1}$. In the exposed case, a shorter chain diluent thiol was used, $\mathrm{HSC}_{11} \mathrm{COOH}$ and this gave rise to a standard rate constant of $20 \mathrm{~s}^{-1}$. This increase in rate is double that observed in this study for a comparable change in chain length. This difference is attributed to the different terminal groups on the diluent thiols used in the two data sets. In this work the terminal group of the diluent thiol was $-\mathrm{OH}$ instead of $-\mathrm{COOH}$ used by Finklea. The exact influence of the terminal group is not very clear, but the change in rate constant with respect to diluent chain could be equivalent considering the fact that the terminal groups are different and the redox molecules are different.

Measurements of the standard rate constant of an Os-Cl complex on a $100 \%$ $\mathrm{HS}\left(\mathrm{CH}_{2}\right)_{15} \mathrm{COOH}$ SAM yielded a value of about $10 \mathrm{~s}^{-1}$, over the entire $\mathrm{pH}$ range. ${ }^{22}$ This compound was used as a control for the aquo complex since it undergoes pure electron transfer over the entire $\mathrm{pH}$ scale. The standard rate constants for the $\mathrm{Os}(\mathrm{L})(\mathrm{L}=\mathrm{OH}$, $\mathrm{H}_{2} \mathrm{O}$ ) on the thicker SAM approach that value at the $\mathrm{pH}$ extremes, suggesting that pure electron transfer is the dominant mechanism at these $\mathrm{pH}$ extremes.

In order to compare the data obtained with the control compound to that obtained using the model Os(L) mentioned above, it is imperative that the electronic coupling 
between the redox molecule and the electrode is understood, as well as the reorganization energies of the various complexes. Kinetic data for the Os-Cl complex yield symmetrical Tafel plots and a reorganization energy of $0.7 \pm 0.1 \mathrm{eV}$ for both the $\mathrm{Os}^{\mathrm{II}}$ and the $\mathrm{Os}{ }^{\mathrm{III}}$ forms.

\subsubsection{Slope vs $\mathrm{pH}$.}

In this study, reorganization energies were calculated from the slopes of the plot of $\alpha$ vs $\eta$ (Figure 6.6). Figure 6.13 shows the slope of both cathodic (diamonds) and anodic (squares), based on equivalent analyses like that in Figure 6.6 at all pHs.

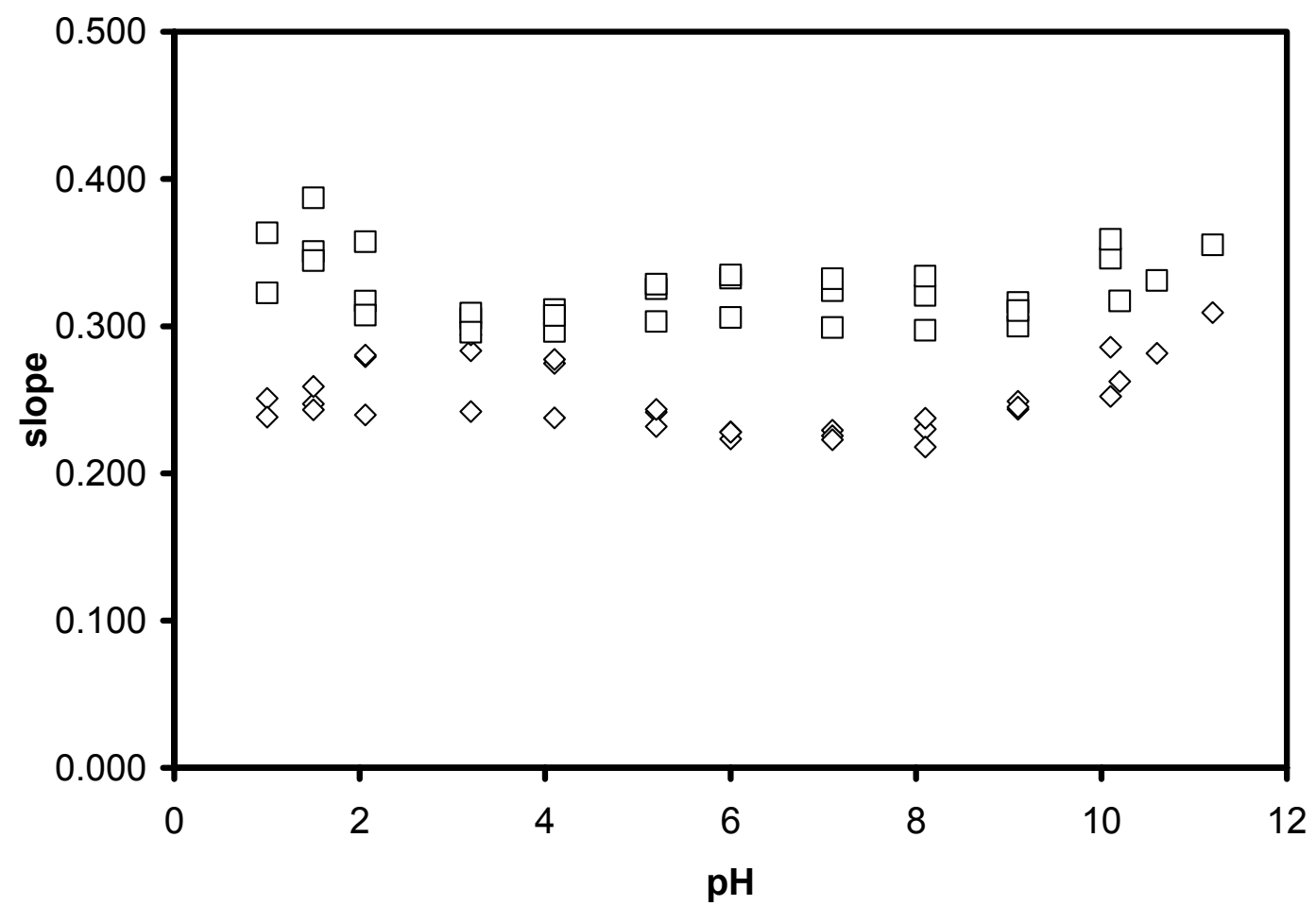

Figure 6.13. Slope (units $\mathrm{V}^{-1}$ ) of the anodic branch (squares) and cathodic branch (diamonds) obtained from the plot of $\alpha(0)$ vs. $\eta$ in $\mathrm{H}_{2} \mathrm{O}$. 
The cathodic slopes were always smaller than anodic slopes in agreement with data shown in Figure 6.6. Because of the inverse relationship between the reorganization energy and the slope, cathodic slopes translate into larger reorganization energies and anodic slopes translate into smaller reorganization energies. It is not easy to tell the dependence of slope on $\mathrm{pH}$ from Figure 6.13. A clearer picture is obtained from the plot of reorganization energy against $\mathrm{pH}$, Figure 6.14.

\subsubsection{Reorganization energy vs $\mathrm{pH}$.}

From Figure 6.14, the reorganization for $\mathrm{Os}^{\mathrm{III}}$ is less sensitive to $\mathrm{pH}$ than the reorganization for Os ${ }^{\mathrm{II}}$. This observation is much more evident between $\mathrm{pH} 4$ and 8 .

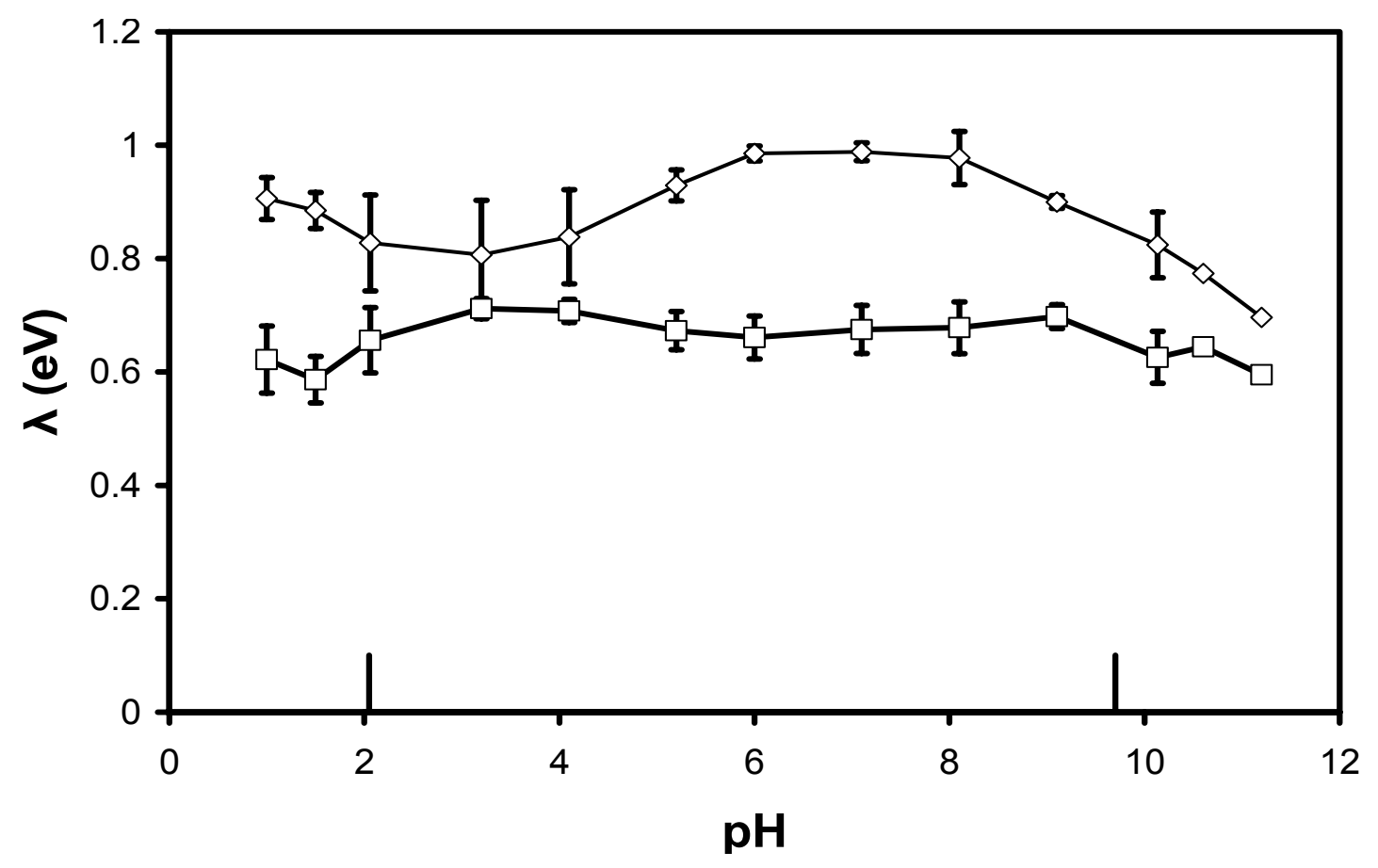

Figure 6.14. Variation of reorganization energy for $\mathrm{Os}^{\mathrm{II}}$ (diamonds) and $\mathrm{Os}{ }^{\mathrm{III}}$ (squares) as a function of $\mathrm{pH}$ in water. Each symbol is an average of three experiments and bars represent a single standard deviation. The data were generated using slope values in Figure 6.13. 
A baffling feature is that the reorganization energy for the $\mathrm{Os}^{\mathrm{II}}$ state $(0.9 \pm 0.1 \mathrm{eV})$ is significantly larger than that for $\mathrm{Os}^{\mathrm{III}}(0.6 \pm 0.1 \mathrm{eV})$. This behavior was not expected especially considering the fact that the outer sphere reorganization energies of the two oxidation states are the same. The outer sphere reorganization energy is independent of the oxidation state of the metal center. The major difference then would be in the inner sphere component of the reorganization energy. Since Os ${ }^{\mathrm{III}}$ has higher charge than Os ${ }^{\mathrm{II}}$, using electrostatic arguments, the bond force constants for $\mathrm{Os}{ }^{\mathrm{III}}(\mathrm{OH})$ is expected to be higher than for $\mathrm{Os}^{\mathrm{II}}\left(\mathrm{OH}_{2}\right)$, leading to larger inner sphere reorganization energy for $\mathrm{Os}^{\mathrm{III}}$ than that for Os ${ }^{\mathrm{II}}$. A similar argument was used by Weaver and Hupp to explain the observed kinetic behavior of aquated $\mathrm{Cr}^{2+3+}$ and $\mathrm{Eu}^{2+/ 3+}$. In their study, they observed asymmetric Tafel plots and an $\alpha(0)$ greater than 0.5 . Investigations based on metal-ligand bond length data revealed that force constants and bond lengths changed substantially between the oxidized and reduced species. This change in bond length resulted in different reorganization energies between the oxidation states, the higher oxidation state having the higher reorganization energy than the lower oxidation state. To calculate the total reorganization energy, Hupp and Weaver assumed the same value for the outer sphere reorganization of the oxidized and reduced species, since this parameter is independent of oxidation state. The inner sphere reorganization energy was calculated based on known values for bond vibration frequency and changes in bond lengths between the oxidized and reduced species. For the $\mathrm{Cr}^{+2 /+3}$ redox couple, values obtained were $310 \mathrm{~kJ} / \mathrm{mol}$ for the +3 state and $197 \mathrm{~kJ} / \mathrm{mol}$ for the +2 state, which correspond to 3.2 $\mathrm{eV}$ and $2.0 \mathrm{eV}$ respectively. Their results were consistent with Tafel plots having more curved anodic branch than the cathodic branch. 
In this study, the observed trend was opposite from what Hupp and Weaver observed. The reduced species had a higher reorganization energy than the oxidized species. The cause of this anomalous behavior cannot be explained.

It is also evident that the two plots approach each other at $\mathrm{pHs}$ near the $\mathrm{pK} \mathrm{a}$. At intermediate $\mathrm{pHs}$ (between $\mathrm{pK}_{\mathrm{a} 1}$ and $\mathrm{pK}_{\mathrm{a} 2}$ ), the difference between the two curves is more pronounced. This behavior seems to correlate well with the behavior of the standard rate constant, Figure 6.7. In particular, the reorganization energy for the Os ${ }^{\mathrm{II}}$ seems to be controlling the behavior of the standard rate constant. Large reorganization energy values for $\mathrm{Os}^{\mathrm{II}}$ correspond to lower standard rate constant, especially at intermediate $\mathrm{pH}$ (4 to 8 ). Experiments carried out in deuterated electrolytes revealed a similar variation of reorganization energy with $\mathrm{pD}$, Figure 6.15 . Within experimental uncertainty, the anodic reorganization follows almost exactly the same trend observed in light water. However, the reorganization energy for $\mathrm{Os}^{\mathrm{II}}$ is even more sensitive to $\mathrm{pD}$ than $\mathrm{pH}$, especially around $\mathrm{pD} 4-8$. Moreover, at $\mathrm{pD}$ greater than $\mathrm{pK}_{\mathrm{a} 2}$, the two curves converge at higher values than observed in light water. Higher reorganization energies for $\mathrm{Os}^{\mathrm{II}}$ in $\mathrm{D}_{2} \mathrm{O}$ decreases the standard rate constant even more leading to the behavior observed in Figure 6.8 (isotope effect), where the standard rate constants measured in heavy water are lower than those measured in light water. This observation further strengthens the argument that the reorganization energy for $\mathrm{Os}^{\mathrm{II}}$ is the dominant parameter controlling the standard rate constant. 


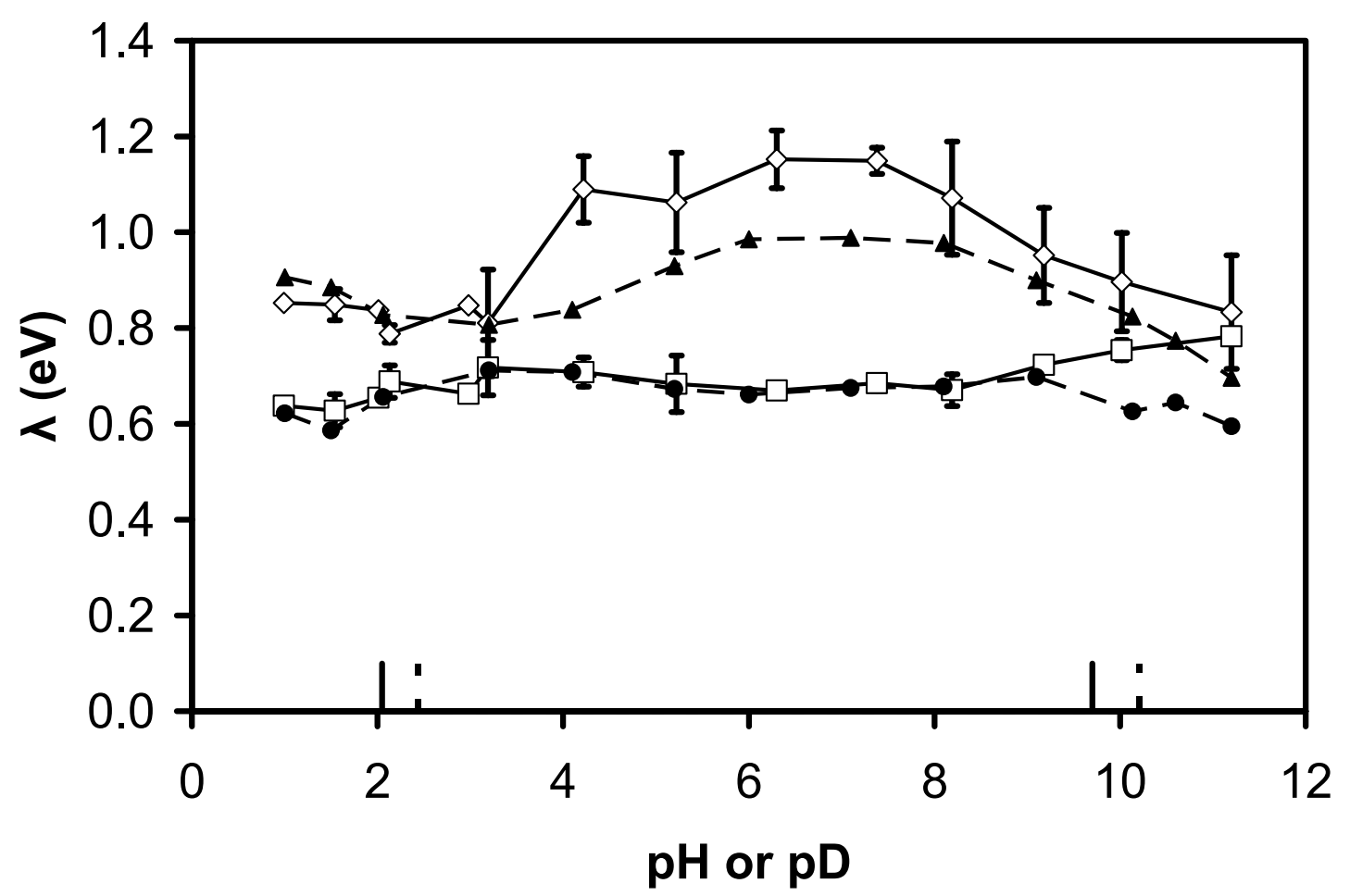

Figure 6.15. Comparison of reorganization energies for $\mathrm{Os}^{\mathrm{III}}$ in $\mathrm{D}_{2} \mathrm{O}$ (open squares) vs $\mathrm{Os}^{\mathrm{III}}$ in $\mathrm{H}_{2} \mathrm{O}$ (solid circles) and $\mathrm{Os}^{\mathrm{II}}$ in $\mathrm{D}_{2} \mathrm{O}$ (open diamonds) vs. $\mathrm{Os}^{\mathrm{II}}$ in $\mathrm{H}_{2} \mathrm{O}$ (solid triangles). Each of the data points is an average of three different experiments and bars represent a single standard deviation. 


\section{CHAPTER 7: DISCUSSION.}

\subsection{Proposed mechanism.}

It is clear from the above observations that the stepwise model cannot explain the observed kinetic behavior of the osmium system, $\left[\mathrm{Os}(\mathrm{bpy})_{2}(\mathrm{py})\left(\mathrm{OH}_{2}\right)\right]^{2+}$. In particular, the deep minimum in the plot of $\log \left(\mathrm{k}_{\mathrm{s}}\right)$ vs $\mathrm{pH}$ (Figure 6.7) and the oscillatory behavior of the transfer coefficient predicted by theory (Figure 6.9) were not observed experimentally. Using the square scheme in Chapter 3, Figure 3.1 and assuming a reorganization energy of $0.9 \mathrm{eV}$ for all four osmium species, and the same standard rate constant, the standard rate constant predicted by the stepwise model would be 60 times smaller than the standard rate constants at the $\mathrm{pH}$ extremes where the plots level off and become independent of $\mathrm{pH}$ (Figure 6.7). Results from this study indicate that the standard rate decreased only by a factor of four.

The diagonal in Figure 3.1 describes the concerted mechanism. In the concerted mechanism, both the proton and electron should appear in the rate determining step.

$\mathrm{Os}^{\mathrm{III}}(\mathrm{OH})+\mathrm{e}^{-}+\mathrm{H}^{+} \leftrightarrow \mathrm{Os}^{\mathrm{II}}\left(\mathrm{OH}_{2}\right)$

However, this Equation can be misleading as it predicts a cathodic rate constant that is $\mathrm{pH}$ dependent and an anodic rate constant that is independent of $\mathrm{pH}$, which is not observed experimentally.

We hypothesize that the reduction step is not a direct reverse of the oxidation step as commonly assumed for most redox species. The proposed mechanism is shown by the square scheme II in Figure 7.1. Because reduction is not a direct reverse of the oxidation process, it is possible to have different reorganization energies for the two processes. 
However, the two processes are thermodynamically equivalent; otherwise large peak splittings would be seen even at the lowest scan rates.

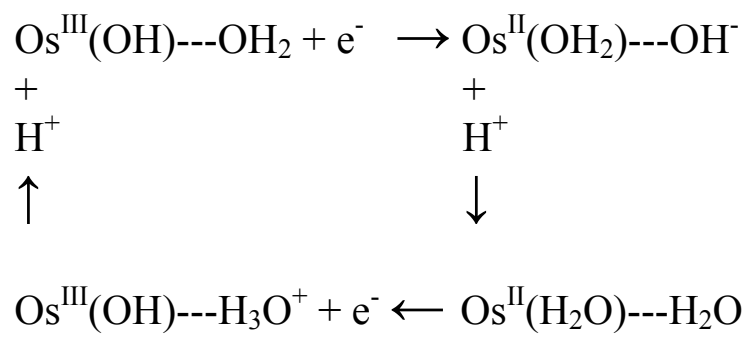

Figure 7.1. Square Scheme II: Proposed mechanism for the oxidation and reduction reaction of $\left[\mathrm{Os}(\mathrm{bpy})_{2}(\mathrm{py})\left(\mathrm{OH}_{2}\right)\right]^{2+}$

The reaction partner for proton transfer is likely to be water at all $\mathrm{pH}$ due to its high concentration $(55 \mathrm{M})$ compared to $\mathrm{H}^{+}$and $\mathrm{OH}^{-}$. Based on this assumption, the reduction step would be better represented as:

$\mathrm{Os}^{\mathrm{III}}(\mathrm{OH})---\mathrm{OH}_{2}+\mathrm{e} \longrightarrow \mathrm{Os}^{\mathrm{II}}\left(\mathrm{OH}_{2}\right)---\mathrm{OH}$

In the reactant, the water molecule is assumed to partner with the hydroxide ligand through strong hydrogen bonding.

Since there is a finite delay between the reduction and oxidation steps even at fast scan CVs, the proton transfer causes the product molecule $\left(\mathrm{OH}^{-}\right.$or $\left.\mathrm{H}_{3} \mathrm{O}^{+}\right)$to be replaced by water prior to the scan in the reverse direction. As a result, better representation of the rate-determining anodic step will be:

$\mathrm{Os}^{\mathrm{II}}\left(\mathrm{OH}_{2}\right)---\mathrm{OH}_{2} \longrightarrow \mathrm{Os}^{\mathrm{III}}(\mathrm{OH})---\mathrm{OH}_{3}{ }^{+}+\mathrm{e}^{-}$

The weak dependence on $\mathrm{pH}$ of the kinetic parameters suggests that the solvent (water), as opposed to the proton or hydroxide ion, is playing a major role in the proton 
donor/acceptor realm. The transferring proton is not coming from the hydronium ion $\left(\mathrm{H}_{3} \mathrm{O}^{+}\right)$but from the solvent. The dotted lines show the close association between the respective species through hydrogen bonding. Based on this mechanism, it is clear that each of the rate determining steps does not have $\mathrm{pH}$ explicitly defined in the rate law. Consequently, to a first approximation, the standard rate constant will be expected to have at most a weak dependence on $\mathrm{pH}$.

The experimental data show a weak dependence of the standard rate constant on $\mathrm{pH}$, Figure 6.7. However, slopes of the plot of $\log \left(\mathrm{k}_{\mathrm{s}}\right) \mathrm{vs} \mathrm{pH}$ do not change at $\mathrm{pHs}$ beyond the two $\mathrm{pK}_{\mathrm{a}} \mathrm{s}$. There is a possibility of an alternative rate determining step at low $\mathrm{pH}(\mathrm{pH}$ $\left.<\mathrm{pK}_{\mathrm{a} 1}\right)$ and high $\mathrm{pH}\left(\mathrm{pH}>\mathrm{pK}_{\mathrm{a} 2}\right)$. The suggested mechanism at low $\mathrm{pH}$ should involve the fully protonated species as shown at the bottom of Square Scheme I, Figure 3.1. This reaction is a pure electron transfer step with no accompanying proton transfer:

$\mathrm{Os}^{\mathrm{III}}\left(\mathrm{OH}_{2}\right)---\mathrm{OH}_{2}+\mathrm{e}^{-} \leftrightarrow \mathrm{Os}^{\mathrm{II}}\left(\mathrm{OH}_{2}\right)---\mathrm{OH}_{2}$

Similarly at high $\mathrm{pH}\left(\mathrm{pH}>\mathrm{pK}_{\mathrm{a} 2}\right)$, an equivalent reaction involving the fully deprotonated form of the Os ${ }^{\text {III/II }}$ species can be written:

$\mathrm{Os}^{\mathrm{III}}(\mathrm{OH})---\mathrm{OH}_{2}+\mathrm{e}^{-} \leftrightarrow \mathrm{Os}^{\mathrm{II}}(\mathrm{OH})---\mathrm{OH}_{2}$

This Equation is equivalent to the top step of Square Scheme I, Figure 3.1. Assuming that this step has a different standard rate constant than that in Equation 7.4, the $\log \left(\mathrm{k}_{\mathrm{s}}\right) \mathrm{vs} \mathrm{pH}$ plot, Figure 6.7 would be based on two competing processes at low and high $\mathrm{pH}$. The problem with this kind of scenario, however, is that, since these two processes are based on a simple electron transfer, the standard rate constant should be constant and $\alpha(0)$ 
should be equal to 0.5 in this region. The experimental data do not support these predictions.

Recent studies in homogeneous solutions have focused on a concerted mechanism in which the proton and electron transfer steps are coupled rather than separated. ${ }^{41,47,53,56}$ This means that the two transfers occur simultaneously, rather than as two distinct steps. It would be interesting to see if the concerted model can explain these observations. A more direct way to test the concerted model is to look at the effect of replacing the proton with its heavier analogue, the deuteron. Since the stepwise model predicts that the proton transfer step is at equilibrium, that is, not rate limiting, replacing the proton with the deuteron will have little or no effect on the standard rate constant. For the concerted mechanism, however, the kinetic isotope effect is dependent on electron and proton tunneling distance, and can range from unity (no kinetic isotope) effect to very large values. ${ }^{56,79}$ In general, proton or deuteron tunneling over large distances is associated with large kinetic isotope effect while proton or deuteron tunneling over short distances is associated with small kinetic isotope effect.

Replacing the proton with the deuteron did not have a significant effect on the thermodynamic properties or $\alpha(0)$ of the osmium complex. However, the standard rate constant was decreased by a factor of two, especially at intermediate $\mathrm{pHs}$. This small change in the standard rate constant suggests a small kinetic isotope effect. This also suggests that the proton tunneling distance is small. If tunneling distance was large, then a large kinetic isotope effect would be expected as the heavier deuteron traverses through large distances. ${ }^{43,47,53,56,79}$ 


\subsection{Conclusion:}

The osmium complex, $\left[\mathrm{Os}(\mathrm{bpy})_{2}(\mathrm{py})\left(\mathrm{OH}_{2}\right)\right]^{2+}$ was a good model compound to obtain both thermodynamic and kinetic information for PCET processes. Measurements were done for the entire $\mathrm{pH}$ region (low, medium and high $\mathrm{pHs}$ ). Thermodynamically, the osmium system behaves as predicted by the square scheme for a $1 \mathrm{e}, 1 \mathrm{H}$ system. The plot of formal potential as a function of $\mathrm{pH}$ (Figure 6.2), fits the predictions of the square scheme very well. In the regions $\mathrm{pH}<\mathrm{pK}_{\mathrm{a} 1}$ and $\mathrm{pH}>\mathrm{pK}_{\mathrm{a} 2}$, where pure electron transfer is expected, the plot is independent of $\mathrm{pH}$. In the regions between the two $\mathrm{pK}_{\mathrm{a}} \mathrm{s}$, a Nernstian dependence of formal potential on $\mathrm{pH}$ was observed as expected. The thermodynamic parameters obtained here $\left(\mathrm{pK}_{\mathrm{a} 1}=2.05, \mathrm{pK}_{\mathrm{a} 2}=9.7, \mathrm{E}_{1}^{0}=0.41 \mathrm{~V}, \mathrm{E}_{2}^{0}=-\right.$ $0.04 \mathrm{~V})$ compare well with Meyer's data for $\left[\mathrm{Os}(\mathrm{tpy})(\mathrm{bpy})\left(\mathrm{OH}_{2}\right)\right]^{2+}$ freely diffusing in solution $\left(\mathrm{pK}_{\mathrm{a} 1}=2, \mathrm{pK}_{\mathrm{a} 2}=8, \mathrm{E}^{0}{ }_{1}=0.37 \mathrm{~V}, \mathrm{E}^{0}{ }_{2}=0.05 \mathrm{~V}\right)$. There is also a fair agreement with Haddox's data for $\left[\mathrm{Os}(\mathrm{bpy})_{2}(\mathrm{py}) \mathrm{OH}_{2}\right]^{2+}\left(\mathrm{pK}_{\mathrm{a} 1}=2.4, \mathrm{pK}_{\mathrm{a} 2}=9.3, \mathrm{E}^{0}{ }_{1}=0.3 \mathrm{~V}, \mathrm{E}_{2}^{0}=-\right.$ $011 \mathrm{~V})$. In deuterated electrolytes, the thermodynamic data are $\mathrm{pK}_{\mathrm{a} 1}=2.2, \mathrm{pK}_{\mathrm{a} 2}=10.2$, $\mathrm{E}^{0}{ }_{1}=0.42 \mathrm{~V}, \mathrm{E}^{0}{ }_{2}=-0.05 \mathrm{~V}$. Studies with the redox molecule in solution using a bare gold electrode yielded the following results: $\mathrm{pK}_{\mathrm{a} 1}=2.15, \mathrm{pK}_{\mathrm{a} 2}=9.7, \mathrm{E}_{1}^{0}=0.33 \mathrm{~V}, \mathrm{E}_{2}^{0}=-0.13$ V. These results show that thermodynamic properties are fairly consistent and Laviron's formulations can accurately describe the thermodynamic properties of PCET reactions.

The kinetic predictions of the stepwise model could not be verified experimentally; therefore, it is ruled out as a mechanism of PCET reactions. In particular, both the plot of $\log \left(\mathrm{k}_{\mathrm{s}}\right)$ vs $\mathrm{pH}$ and $\alpha(0)$ vs $\mathrm{pH}$ were weakly dependent on $\mathrm{pH}$ contrary to predictions of the stepwise model. The $\alpha(0)$ vs $\mathrm{pH}$ plot did not show the predicted 
oscillatory behavior with $\mathrm{pH}$ and the $\log \left(\mathrm{k}_{\mathrm{s}}\right) \mathrm{vs} \mathrm{pH}$ plot did not show breaks in the standard rate constant at extreme $\mathrm{pHs}$ or a deep minimum at intermediate $\mathrm{pH}$.

An alternative method, the concerted mechanism, has been proposed to explain the observed kinetic behavior. However, the concerted mechanism does not explicitly explain the observed kinetic trends either. In particular, two key observations could not be explicitly explained. First, the reorganization energy for the oxidized species is always lower than that of the reduced species. Second, there is a sudden break on the plot of the standard rate constant against $\mathrm{pD}$ between $\mathrm{pD} 3$ and 4 . To our knowledge, this is the first report on such observations. The kinetic isotope effect is not large, suggesting that the tunneling distances traversed by the proton or deuteron between donor and acceptor sites is short. Also the strong connection between the standard rate constant vs $\mathrm{pH}$ or $\mathrm{pD}$ and changes in $\lambda$ for Os ${ }^{\mathrm{II}}$ (Figure 6.8 and 6.15) suggest that the reorganization energy for Os ${ }^{\mathrm{II}}$ plays a key role in the kinetic behavior of the osmium $(1 \mathrm{e} 1 \mathrm{H})$ system. This observation could not be explained.

The absence of a fully developed theoretical formulation has limited the application of the concerted mechanism to explain these results. More work still needs to be done in the development of a sound theoretical background to explain the kinetics of PCET reactions in order to make meaningful theoretical predictions. Experimental work to back up theory is also required especially with more biologically relevant redox systems such as catechols and quinones. This could find applications in the development of electrocatalysts for PCET reactions that can be used for in-situ monitoring of neurotransmitters such as dopamine in living organisms. 


\subsection{Future Work}

Although the osmium system was a good 1e,1H system which permitted the determination of both thermodynamic and kinetic parameters, this work is an open ended project, the study is far from complete. Several different directions can still be pursued. The initial focus should continue to be on understanding simple 1e,1H models, before more complex multi-proton and multi-electron systems can be examined. It will be interesting to see the effect of changing the proton transfer site by replacing the $\mathrm{H}_{2} \mathrm{O}$ molecule with other proton transfer sites such as imidazole, benzimidazole or amine groups. Other metal centers such as ruthenium could be examined as well.

With the osmium aquo system, studies at higher $\mathrm{pH}$ were limited by the fact that a second wave began to merge with the wave of interest. Low $\mathrm{pH}$ were studies were limited by the fact that the redox molecule was easily lost, possibly due to the loss of the self assembled monolayer through oxidation. This problem was encountered mainly at higher scan rates. It will be interesting to find out if the same problem will be observed with other redox species.

Another worrying problem encountered with the osmium aquo complex was the presence of an extra wave which was independent of $\mathrm{pH}$. This wave is more significant at low pHs. The cause of this extra wave is not known. It is possible that two aminomethylpyridine molecules substitute at the metal center and displace the water ligand. This can occur if the concentration of 4-Aminomethylpyridine is high. It will be interesting to look at a situation where the amount of water is in large excess compared to the amount of 4-Aminomethylpyridine added. It will also be interesting to monitor the $\mathrm{pH}$ 
of the solution after dissolving the carbonato complex as well as 4-Aminomethylpyridine to find the optimum conditions for the formation of $\left[\mathrm{Os}(\mathrm{bpy})_{2}(\mathrm{py})\left(\mathrm{OH}_{2}\right)\right]^{2+}$.

Finally, proton-coupled electrons transfer studies can also be carried out with more biologically relevant molecules such as catechols, quinones and flavins. 


\section{References}

(1) Faulkner, L. R.; Bard, A. J. Electrochemical Methods: Fundamentals and Applications,; John Wiley and Sons, 2002.

(2) Liu, B.; Bard, A. J.; Mirkin, M. V.; Creager, S. E. Journal of the American Chemical Society 2004, 126, 1485-1492.

(3) Harris, D. C. Quantitative Chemical Analysis, 6th ed.; W.H. Freeman and Company: New York, 2003.

(4) Terrettaz, S.; Becka, A. M.; Traub, M. J.; Fettinger, J. C.; Miller, C. J. Journal of Physical Chemistry 1995, 99, 11216-11224.

(5) Robinson, D. B.; Chidsey, C. E. D. Journal of Physical Chemistry B 2002, 106, 10706-10713.

(6) Smalley, J. F.; Finklea, H. O.; Chidsey, C. E. D.; Linford, M. R.; Creager, S. E.; Ferraris, J. P.; Chalfant, K.; Zawodzinsk, T.; Feldberg, S. W.; Newton, M. D. Journal of the American Chemical Society 2003, 125, 2004-2013.

(7) Miller, C. J. In Physical Electrochemistry: Principles, Methods and applications; Rubinstein, Israel, Ed.; Marcel Dekker.: New York, 1995, pp p. 27-79.

(8) Marcus, R. A. Journal of Electroanalytical Chemistry 1997, 438, 251-259.

(9) Ravenscroft, M. S.; Finklea, H. O. Journal of Physical Chemistry 1994, 98, 38433850.

(10) Finklea, H. O. Journal of Electroanalytical Chemistry 2001, 495, 79-86.

(11) Weber, K.; Creager, S. E. Analytical Chemistry 1994, 66, 3164-3172.

(12) Haddox, R. M., Dissertation, West Virginia University, Morgantown, 2002. 
(13) Hupp, J. T.; Weaver M. J. Journal of Physical Chemistry 1984, 88, 6128-6135.

(14) Constentin, C.; Robert, M.; Saveant, J-M. Journal of Electroanalytical Chemistry 2006, 558, 197-206.

(15) Hanshew, D. D.; Finklea, H. O. Journal of the American Chemical Society 1992. $114,3173-3181$.

(16) Finklea, H. O.; Hanshew, D. D. Journal of Electroanalytical Chemistry 1993, $347,327-340$.

(17) Marcus, R. A.; Gosavi, S. Journal of Physical Chemistry 2000, 104, 2067 - 2072.

(18) Love, J. C.; Estroff, L. A.; Kriebel, J. K.; Nuzzo, R. G.; Whitesides, G. Chemical Reviews 2005, 105, 1103-1169.

(19) Colorado, R. Jr.; Lee, T. R. http://www.chem.uh.edu/Faculty/lee/Research/pdf/ 2001/Encyclo\%20Mat\%20(2001)\%209332.pdf

(20) Pradeep, T. RESONANCE journal of science education 1999, 4, 53-62.

(21) Bain, C. D.; Troughton, E. B.; Tao, Y. T.; Evall, J.; Whitesides; G.M., Nuzzo, G.G. Journal of the American Chemical Society 1989, 111, 321-335.

(22) Yamada, R.; Wano, H.; Uosaki, K. Langmuir 2000, 16, 5523-5525.

(23) Pemberton, J. E.; Schoenfisch, M. H. Journal of the American Chemical Society 1998, 120, $4502-4513$.

(24) Love, J. C.; Wolfe, D. B.; Haasch, R.; Chabinyc, M. L.; Paul, K. E.; Whitesides, G. M.; Nuzzo, R. G. Journal of the American Chemical Society 2003, 125, 2597 2609.

(25) Cavarlhal, R. F.; Freire, R. S.; Kubota, L. T. Electroanalysis 2005, 17, 1251-1259.

(26) Chidsey, C. E. D. Science 1991, 251, 919. 
(27) Sun, L.; Crooks, R. M. Journal of Electrochemical Society 1991, 138, L23-L25.

(28) Kim, Y. T.; MaCarley R. M.; Bard, A. J. Langmuir 1993, 9, pp 1941 - 1944;.

(29) Wang, H.; Chen, S.; Li, L.; Jiang, S. Langmuir 2005, 21, 2633-2636.

(30) Finklea, H. O. Encyclopedia of Electrochemistry 2000, 10.

(31) Finklea, H. O. West Virginia University, 2000, unpublished.

(32) Chidsey, C. E. D.; Bertozzi, C.R.; Putvinski, T.M.; Mujsce, A.M. Journal of the American Chemical Society 1990, 112, 4301 - 4306.

(33) Finklea, H. O. In Electroanalytical Chemistry, Marcel Dekker: New York; Vol. 19, 525 .

(34) Finklea, H. O. Electroanalytical Chemistry 1996, 19, 109-335.

(35) Finklea, H. O.; Liu, L., Ravenscroft, M. S.; Punturi, S. Journal of Physical Chemistry 1996, 100, 18852-18858.

(36) Slowinski, K.; Chamberlain, R.V.; Miller, C. J.; Majda, M. Journal of the American Chemical Society 1997, 119 11910-11919.

(37) Becka, A. M.; Miller, C. J. Journal of Physical Chemistry 1992, 96, 2657-2668.

(38) Cheng, J.; Sàghi-Szabó, G.; Tossell, J. A.; Miller C. J. Journal of the American Chemical Society 1996, 118, 680 - 684.

(39) Laviron, E. Journal of Electroanalytical Chemistry 1981, 124, 1-7.

(40) Cukier, R. I. Journal of Physical Chemistry 1995, 99, 16101-16115.

(41) Cukier, R. I. Journal of Physical Chemistry 1996, 100, 15428-15443.

(42) Decornez, H.; Hammes-Schiffer, S. Journal of Physical Chemistry A 2000, 104, 9370-9384. 
(43) Iordanova, N.; Hammes-Schiffer, S. Journal the American Chemical Society 2002, 124, 4848-4856.

(44) Costentin, C.; Robert, M.; Saveant, J-M. Journal of the American Chemical Society 2004, 126, 16834-16840.

(45) Haddox, R. M.; Finklea, H. O. Journal of Physical Chemistry B 2004, 108, 16941700.

(46) Mayer, J. M.; Rhile, I. Biochimica et Biophysica Acta 2004, 1655, 51-58.

(47) Hatcher, E.; Soudackov, A.; Hammes-Schiffer, S. Chemical Physics 2005, 319, 93-100.

(48) Grimminger, J.; Bartenschlarger, S.; Schmickler, W. Chemical Physics Letters 2005, 416, 316-320.

(49) Belevich, I.; Verkhovsky, M. I.; Wikstrom, M. Nature 2006, 440, 829-832.

(50) Nocera, G.; Cukier, R. I. Annual Reviews of Physical chemistry 1998, 49, 337-369

(51) Soudackov, A.; Hammes-Schiffer, S. Journal of Chemical Physics 2000, 113, 2385-2396.

(52) Lebeau, E. L.; Meyer, T. J.; Binstead, R. A. Journal of the American Chemical Society 2001, 123, 10535-10544.

(53) Hammes-Schiffer, S. Accounts of Chemical Research 2001, 34, 273-281.

(54) Iordanova, N.; Hammes-Schiffer, S. Biochimica et Biophysica Acta 2004, 1655, 29-36.

(55) Cukier, R.I. Biochimica et Biophysica Acta 2004, 1655, 37-44.

(56) Hatcher, E.; Soudackov, A.; Hammes-Schiffer, S. Journal of Physical Chemistry B 2005, 109, 18565-18574. 
(57) Laviron, E. Journal of Electroanalytical Chemistry 1980, 109, 57-67.

(58) Finklea, H. O. Journal of Physical Chemistry B 2001, 105, 8685-8693.

(59) Finklea, H. O.; Haddox, R. M. Physical Chemistry Chemical Physics 2001, 3, 3431-3436.

(60) Albery, J. Electrode kinetics; Clarendon Press: Oxford, 1975.

(61) Buckingham, D. A.; Dwyer, F. P.; Goodwin, H. A. Australian Journal of Chemistry 1964, 17, 325-336.

(62) Takeuchi, K.; Thompson M. S.; Pipes, D. W.; Meyer, T. J. Inorganic Chemistry 1984, 23, 1845-1851.

(63) Dobson, J. C.; Takeuchi, K. J.; Pipes, D. W.; Geselowitz, D. A.; Meyer, T. J. Inorganic Chemistry 1986, 25, 2357-2365.

(64) Kober, E. M.; Caspar, J. V.; Sullivan, B. P.; Meyer, T. J. Inorganic Chemistry 1988, 27, 4587-4598.

(65) Brewer*, K. J.; Richter, M. M. Inorganica Chimica Acta 1991, 180, 125-131.

(66) Sauvage, Jean-Pierre; Collin, J. P.; Chambron, Jean-Claude; Coudret, C.; Balzani, V.; Barigelleti, F.; Cola, L. D.; Flamigni, L. Chemical Reviews 1994, 94, 9931019.

(67) Meyer, T. J.; Huynh, M. H. V. Inorganic Chemistry 2003, 42, 8140 - 8160.

(68) Mirkin, M. V.; Richards, T. C.; Bard, A. J. Journal of Physical Chemistry 1993, 97, $7672-7677$.

(69) Staros, J. V; Wright R. W.; Swingle, D. M. Analytical Biochemistry 1986, 156, 220-222. 
(70) Gilles, M. A.; Hudson A. Q.; Borders Jr, C. L. Analytical Biochemistry 1990, 184, $244-248$.

(71) Sehgal, D.; Vijay, I. K. Analytical Biochemistry 1994, 218, 87-91.

(72) Rowe, G. K.; Carter, M. T.; Richardson, J. N.; Murray, R. W. Langmuir 1995, 11, pp $1797-1806$.

(73) Glasoe, P. K.; Long, F. A. Journal of Physical Chemistry 1960, 64, 188-190.

(74) Covington, A. K.; Paabo, M; Robinson, R. A.; Bates R. G. Analytical Chemistry 1968, $40,700-706$.

(75) Baucke, F. G. K. Journal of Physical Chemistry B 1998, 102, 4835-4841.

(76) Krezel, A; Bal, W. Journal of Inorganic Biochemistry 2004, 98, 161-166.

(77) Tender, L.; Carter M. T.; Murray, R. W. Analytical Chemistry 1994, 66, 3173 -81.

(78) Richardson, J. N.; Peck, S. R.; Larry S. Curtin, L. S.; Tender, L.M.; Terrill, R. H.; Carter, M. T.; Murray, R. W.; Rowe, G. K.; Creager, S .E. Journal of Physical Chemistry 1995, 99, 766 - 772.

(79) Andres, R; M. V, Pak; Hammes-Schiffer, S. Journal of Chemical Physics 2005, $123,64104-108$. 